\title{
Zinc-Enabled Annulation of Trifluorodiazoethane with 2H-Azirines to Construct Trifluoromethyl Pyrazolines, Pyrazoles, and Pyridazines
}

Yue-Ji Chen, ${ }^{\dagger}$ Fa-Guang Zhang, ${ }^{*}, \dagger$ and Jun-An Ma*,†

†Department of Chemistry, Tianjin Key Laboratory of Molecular Optoelectronic Sciences, Frontiers Science Center for Synthetic Biology (Ministry of Education), and Tianjin Collaborative Innovation Center of Chemical Science \& Engineering, Tianjin University, Tianjin 300072; and Joint School of National University of Singapore and Tianjin University, International Campus of Tianjin University, Binhai New City, Fuzhou 350207, P. R. of China.

E-mail:zhangfg1987@tju.edu.cn,majun_an68@tju.edu.cn

\section{Contents}

General procedure for the preparation of $2 \mathrm{H}$-azirine 1 ...........................................S3

General procedure for the preparation of trifluoromethyl pyrazolines 2 ............S4

General procedure for the preparation of 3-trifluoromethyl pyrazoles 5 ...........S14

General procedure for the preparation of 3,5-ditrifluoromethyl pyridazines $6 \mathrm{S17}$

Possible mechanism of 3,5-ditrifluoromethyl pyridazines 6 ...............................S20

General procedure for the preparation of $\alpha, \beta$-unsaturated trifluoromethyl ketone

7. .521

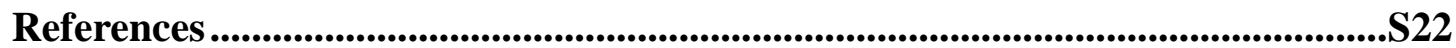

NMR spectra of the related compounds S23

X-Ray crystallographic data S140 


\section{General Considerations}

General Analytical Information. ${ }^{1} \mathrm{H},{ }^{13} \mathrm{C}$ and ${ }^{19} \mathrm{~F}$ were recorded on JEOL AV 600 $\mathrm{MHz} 600 \mathrm{MHz}$ instrument at $600 \mathrm{MHz}\left({ }^{1} \mathrm{H} \mathrm{NMR}\right), 151 \mathrm{MHz}\left({ }^{13} \mathrm{C} \mathrm{NMR}\right)$, as well as $565 \mathrm{MHz}\left({ }^{19} \mathrm{~F}\right.$ NMR). Chemical shifts were reported in ppm down field from internal $\mathrm{Me}_{4} \mathrm{Si}$ and external $\mathrm{CCl}_{3} \mathrm{~F}$, respectively. Multiplicity was indicated as follows: $\mathrm{s}$ (singlet), d (doublet), t (triplet), q (quartet), m (multiplet), dd (doublet of doublet), br (broad). Coupling constants were reported in Hertz $(\mathrm{Hz})$. High resolution mass spectrometry (HRMS) spectra were obtained on a Bruker mior OTOF-QII instrument. X-ray structural analysis was conducted on a Bruker APEX-II CCD instrument.

General Reagents Information. Tetrahydrofuran (THF) and toluene were distilled from sodium/benzophenone prior to use; $\mathrm{CH}_{2} \mathrm{Cl}_{2}$ was distilled from $\mathrm{CaH}_{2} ; \mathrm{MeCN}$, arylboronic acids, palladium acetate and trifluoroacetic acid used are commercially available; diethylzinc 1.0 M solution in hexane was produced by $\mathrm{J} \& \mathrm{~K}$; All purchased reagents were used without further purification. Analytical thin layer chromatography was performed on $0.20 \mathrm{~mm}$ Qingdao Haiyang silica gel plates.

CAUTION: 2,2,2-Trifluorodiazoethane $\left(\mathrm{CF}_{3} \mathrm{CHN}_{2}\right)$ is potentially explosive! Although no accident occurred during the course of this study, stringent safety precautions are necessary for all reactions of $\mathrm{CF}_{3} \mathrm{CHN}_{2}$. The $\mathrm{CF}_{3} \mathrm{CHN}_{2}$ in different solutions were prepared according to the known procedures. ${ }^{1}$ 


\section{General procedure for the preparation of $2 \mathrm{H}$-azirine 1}
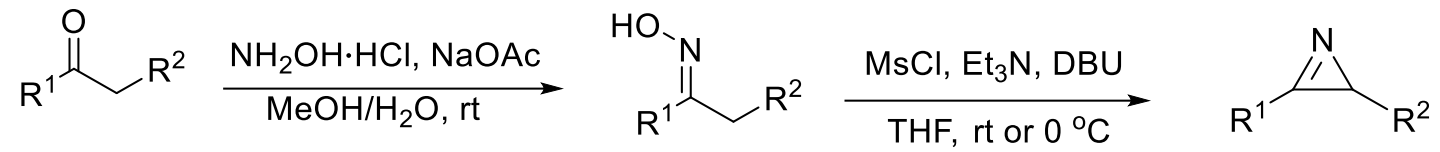

For $1 \mathrm{a} \sim 1 \mathrm{v}$ at $\mathrm{rt}$

$1 \mathrm{w}, 1 \mathrm{x}$, at $0^{\circ} \mathrm{C}$

All the azirines used in this study are known compounds and they are prepared according to reported procedures in the literatures ${ }^{2}$. Step 1: A solvent mixture of $\mathrm{MeOH} / \mathrm{H}_{2} \mathrm{O}$ (20:1) was added to a mixture of ketone (10 mmol, 1 equiv), $\mathrm{NH}_{2} \mathrm{OH} \cdot \mathrm{HCl}(1.5$ equiv), and sodium acetate (1.5 equiv) in a round-bottom flask. The resulting solution was stirred at room temperature, and the reaction was monitored by TLC. After the reaction was completed, the solvent was removed in vacuo and $\mathrm{CH}_{2} \mathrm{Cl}_{2}(30 \mathrm{~mL})$ was then added. The mixture was washed with saturated $\mathrm{NaHCO}_{3}$ solution, followed by brine. The organic phase was dried over anhydrous $\mathrm{MgSO}_{4}$ and the filtrate was concentrated in vacuo to afford oxime as the residue, which was used directly for the next step.

Step 2: To a solution of crude oxime (1 equiv, from step 1) in dry THF (30 mL) were added triethylamine (1.5 equiv) and methanesulfonyl chloride (1.5 equiv) sequentially at room temperature or at $0{ }^{\circ} \mathrm{C}$. The solution became cloudy after the addition of methanesulfonyl chloride. The resulting mixture was stirred for $30 \mathrm{~min}$, and DBU (1.5 equiv) was then added over $1 \mathrm{~min}$. After stirring for an additional $30 \mathrm{~min}$, the reaction mixture was passed through a pad of silica gel and washed with $\mathrm{Et}_{2} \mathrm{O}$ or ethyl acetate $(30 \mathrm{~mL} \times 3)$. The filtrate was concentrated in vacuo, and the residue was purified by column chromatography using petroleum ether and ethyl acetate (20:1) as an eluent to afford the $2 H$-azirine 1a-1x. 
General procedure for the preparation of trifluoromethyl pyrazolines 2

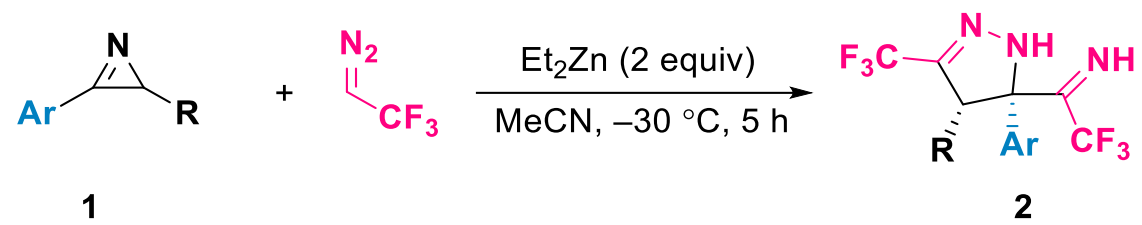

To a $10 \mathrm{~mL}$ Schlenk tube with a magnetic stirring bar, $0.2 \mathrm{mmol}$ of $2 H$-azirine 1 was added. Then, the tube was degassed and refilled with Argon for 3 times. At $-30{ }^{\circ} \mathrm{C}$, a stock solution of $\mathrm{CF}_{3} \mathrm{CHN}_{2}$ in $\mathrm{MeCN}(0.5 \mathrm{M}, 2 \mathrm{~mL}, 5.0$ equiv) was added under Argon. Then Et $2 \mathrm{Zn}(1.0 \mathrm{M}$ in Hexane, $0.4 \mathrm{~mL}, 2.0$ equiv) was added dropwise at $-30{ }^{\circ} \mathrm{C}$ and reacted at $-30{ }^{\circ} \mathrm{C}$ for 5 hours. After the reaction was completed (monitored by TLC), it was quenched with saturated $\mathrm{NH}_{4} \mathrm{Cl}$. The resulting suspension was extracted with DCM $(3 \times 10 \mathrm{~mL})$, then the organic extracts were combined, dried over $\mathrm{MgSO}_{4}$, filtered and concentrated in vacuo. After being concentrated, the residue was purified by flash column chromatography on silica gel (eluting with petroleum ether/ethyl acetate $=50 / 1)$ to give the product 2 .

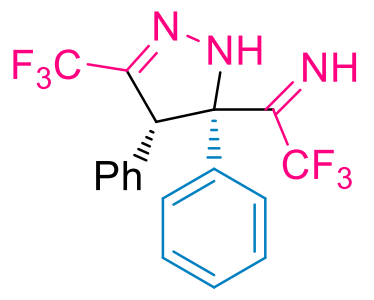

\section{1-(4,5-Diphenyl-3-(trifluoromethyl)-4,5-dihydro-1H-pyraz} ol-5-yl)-2,2,2-trifluoroethanimine (2a): white solid; $63.1 \mathrm{mg}$; $82 \%$ yield; for gram-scale reaction, $1.0 \mathrm{~g}$ of $\mathbf{1 a}$ was used, and $1.7 \mathrm{~g}$ of $\mathbf{2 a}$ was obtained in $85 \%$ yield; m.p. $123-125^{\circ} \mathrm{C} ; \mathrm{R}_{\mathrm{f}}=$ $0.50(\mathrm{PE} / \mathrm{EA}=10 / 1) ;{ }^{1} \mathbf{H}$ NMR (600 MHz, $\left.\mathbf{C D C l}_{3}\right) \delta 11.13(\mathrm{~s}, 1 \mathrm{H}), 7.13-7.08(\mathrm{~m}$, 5H), $7.05-7.01(\mathrm{~m}, 3 \mathrm{H}), 6.92-6.85(\mathrm{~m}, 2 \mathrm{H}), 6.77(\mathrm{~s}, 1 \mathrm{H}), 5.57(\mathrm{~s}, 1 \mathrm{H}) .{ }^{13} \mathbf{C}$ NMR $\left(\mathbf{1 5 1} \mathbf{M H z}, \mathbf{C D C l}_{3}\right) \delta 166.0(\mathrm{q}, J=29.1 \mathrm{~Hz}), 145.9(\mathrm{q}, J=36.9 \mathrm{~Hz}), 133.1(\mathrm{~s}), 132.4$ (s), 129.6 (s), 128.4 (s), 128.3 (s), 128.2 (s), 127.8 (s), 126.7 (d, J = 20.8 Hz), 120.5 (q, $J=271.1 \mathrm{~Hz}), 118.1(\mathrm{q}, J=285.3 \mathrm{~Hz}), 79.9$ (s), 56.1 (s). ${ }^{19}$ F NMR (565 MHz, $\left.\mathbf{C D C l}_{3}\right) \delta-64.21(\mathrm{~s}, 3 \mathrm{~F}),-66.04(\mathrm{~s}, 3 \mathrm{~F})$. HRMS (ESI-TOF) $\mathbf{m} / \boldsymbol{z}:[\mathrm{M}+\mathrm{H}]$ calcd for $\mathrm{C}_{18} \mathrm{H}_{14} \mathrm{~N}_{3} \mathrm{~F}_{6} 386.1092$, found 386.1097.

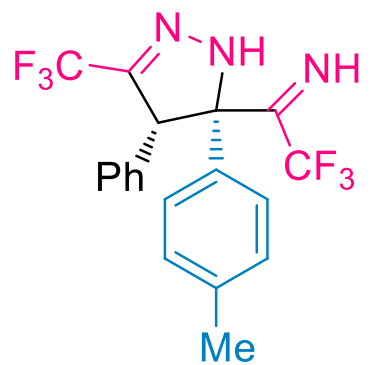

2,2,2-Trifluoro-1-(4-phenyl-5-(p-tolyl)-3-(trifluoromethyl)4,5-dihydro-1H-pyrazol-5-yl)ethanimine (2b): white solid; 
$65.4 \mathrm{mg} ; 82 \%$ yield; m.p. $120-122{ }^{\circ} \mathrm{C} ; \mathrm{R}_{\mathrm{f}}=0.50(\mathrm{PE} / \mathrm{EA}=10 / 1) ;{ }^{1} \mathbf{H}$ NMR $(600$ MHz, $\left.\mathbf{C D C l}_{3}\right) \delta 11.09$ (s, 1H), $7.13-7.02(\mathrm{~m}, 5 \mathrm{H}), 6.82$ (d, J=8.0 Hz, 2H), 6.75 (d, $J=8.1 \mathrm{~Hz}, 2 \mathrm{H}), 6.68(\mathrm{~s}, 1 \mathrm{H}), 5.50(\mathrm{~s}, 1 \mathrm{H}), 2.14(\mathrm{~s}, 3 \mathrm{H}) .{ }^{13} \mathbf{C}$ NMR (151 MHz, CDCl $\left.\mathbf{3}\right)$ $\delta 166.1(\mathrm{q}, J=29.1 \mathrm{~Hz}), 146.2(\mathrm{q}, J=36.6 \mathrm{~Hz}), 137.4(\mathrm{~s}), 133.2(\mathrm{~s}), 129.4(\mathrm{~s}), 129.2$ (s), 128.9 (s), 128.3 (s), 128.3 (s), 126.6 (s), 120.5 (q, $J=271.1 \mathrm{~Hz}), 118.1$ (q, $J=$ 285.4 Hz), 79.7 (s), 55.84 (s), 21.1 (s). ${ }^{19}$ F NMR (565 MHz, CDCl $) \delta-64.21$ (s, 3F), -66.04 (s, 3F). HRMS (ESI-TOF) $m / z:[\mathrm{M}+\mathrm{H}]$ calcd for $\mathrm{C}_{19} \mathrm{H}_{16} \mathrm{~N}_{3} \mathrm{~F}_{6} 400.1248$, found 400.1247 .

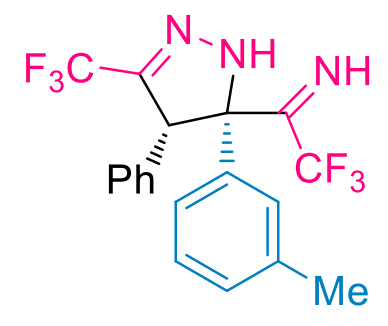

2,2,2-Trifluoro-1-(4-phenyl-5-(m-tolyl)-3-(trifluoromethyl) -4,5-dihydro-1H-pyrazol-5-yl)ethanimine (2c): white solid; $78.2 \mathrm{mg} ; 98 \%$ yield; m.p. $123-124{ }^{\circ} \mathrm{C} ; \mathrm{R}_{\mathrm{f}}=0.50(\mathrm{PE} / \mathrm{EA}=$ 10/1); ${ }^{1} \mathbf{H}$ NMR (600 MHz, $\left.\mathbf{C D C l}_{3}\right) \delta 11.08$ (s, 1H), $7.04-$ $7.00(\mathrm{~m}, 3 \mathrm{H}), 6.97(\mathrm{t}, J=7.9 \mathrm{~Hz}, 1 \mathrm{H}), 6.89-6.81(\mathrm{~m}, 5 \mathrm{H}), 6.65(\mathrm{~s}, 1 \mathrm{H}), 5.50(\mathrm{~s}, 1 \mathrm{H})$, 2.14 (s, 3H). ${ }^{13} \mathbf{C}$ NMR (151 MHz, $\left.\mathbf{C D C l}_{3}\right) \delta 166.1$ (q, $\left.J=28.7 \mathrm{~Hz}\right), 145.7(\mathrm{q}, J=$ $36.7 \mathrm{~Hz}$ ), 138.0 (s), 132.8 (s), 132.4 (s), 129.5 (s), 129.0 (s), 128.2 (s), 128.0 (s), 127.7 (s), 127.2 (s), 123.6 (s), 120.4 (q, $J=271.0 \mathrm{~Hz}), 118.1$ (q, $J=285.3 \mathrm{~Hz}), 79.9$ (s), 56.1 (s), 21.3 (s). ${ }^{19}$ F NMR (565 MHz, CDCl 3 ) $\delta-64.20$ (s, 3F), -65.98 (s, 3F). HRMS (ESI-TOF) $m / z:[\mathbf{M}+\mathbf{H}]$ calcd for $\mathrm{C}_{19} \mathrm{H}_{16} \mathrm{~N}_{3} \mathrm{~F}_{6} 400.1248$, found 400.1244.

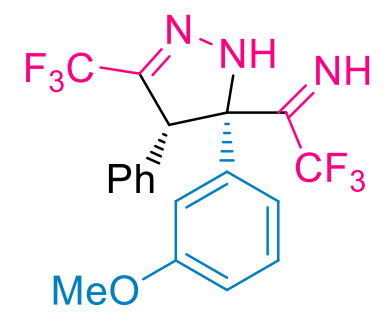

2,2,2-Trifluoro-1-(5-(3-methoxyphenyl)-4-phenyl-3-(trifluo romethyl)-4,5-dihydro-1H-pyrazol-5-yl)ethanimine $\quad(2 \mathrm{~d})$ : white solid; $69.7 \mathrm{mg} ; 84 \%$ yield; m.p. $109-110{ }^{\circ} \mathrm{C} ; \mathrm{R}_{\mathrm{f}}=0.33$ $(\mathrm{PE} / \mathrm{EA}=10 / 1) ;{ }^{1} \mathbf{H}$ NMR (600 MHz, $\left.\mathbf{C D C l}_{3}\right) \delta 11.10$ (s, 1H), $7.07-6.97(\mathrm{~m}, 4 \mathrm{H}), 6.88(\mathrm{dd}, J=6.5,2.9 \mathrm{~Hz}, 2 \mathrm{H}), 6.67(\mathrm{~s}, 1 \mathrm{H}), 6.65-6.62(\mathrm{~m}, 1 \mathrm{H})$, $6.62-6.59(\mathrm{~m}, 2 \mathrm{H}), 5.51(\mathrm{~s}, 1 \mathrm{H}), 3.62(\mathrm{~s}, 3 \mathrm{H}) .{ }^{13} \mathbf{C} \mathbf{N M R}\left(151 \mathrm{MHz}, \mathbf{C D C l}_{3}\right) \delta 166.0$ (q, $J=29.2 \mathrm{~Hz}), 159.5(\mathrm{~s}), 145.8(\mathrm{q}, J=36.7 \mathrm{~Hz}), 134.6(\mathrm{~s}), 132.3$ (s), 129.5 (s), 129.4 (s), 128.1 (s), 127.8 (s), 120.4 (q, $J=271.3 \mathrm{~Hz}), 119.0$ (s), 118.0 (q, $J=284.9$ Hz), 113.8 (s), 112.5 (s), 79.8 (s), 56.1 (s), 55.3 (s). ${ }^{19}$ F NMR (565 MHz, CDCl $) \delta$ 
-64.22 (s, 3F), -65.99 (s, 3F). HRMS (ESI-TOF) $m / z:[\mathrm{M}+\mathrm{H}]$ calcd for $\mathrm{C}_{19} \mathrm{H}_{16} \mathrm{~N}_{3} \mathrm{OF}_{6}$ 416.1198, found 416.1194.

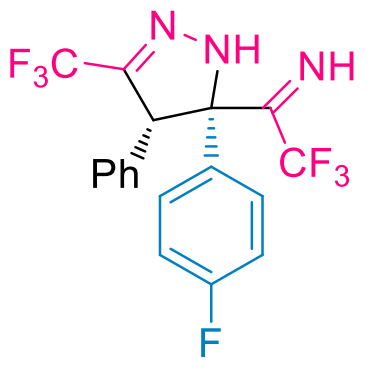

2,2,2-Trifluoro-1-(5-(4-fluorophenyl)-4-phenyl-3-(trifluoro methyl)-4,5-dihydro-1H-pyrazol-5-yl)ethanimine (2e): white solid; $62.9 \mathrm{mg} ; 78 \%$ yield; m.p. $92-94{ }^{\circ} \mathrm{C} ; \mathrm{R}_{\mathrm{f}}=0.48$ $($ PE/EA $=10 / 1) ;{ }^{1} \mathbf{H}$ NMR (600 MHz, $\left.\mathbf{C D C l}_{3}\right) \delta 11.13(\mathrm{~s}$, 1H), $7.09-6.99(\mathrm{~m}, 5 \mathrm{H}), 6.85(\mathrm{~s}, 2 \mathrm{H}), 6.78(\mathrm{t}, J=7.7 \mathrm{~Hz}, 2 \mathrm{H})$,

$6.66(\mathrm{~s}, 1 \mathrm{H}), 5.50(\mathrm{~s}, 1 \mathrm{H}) .{ }^{13} \mathbf{C}$ NMR (151 MHz, CDCl 3$) \delta 165.8(\mathrm{q}, J=29.1 \mathrm{~Hz})$, $162.4(\mathrm{~d}, J=248.3 \mathrm{~Hz}), 146.2(\mathrm{q}, J=37.0 \mathrm{~Hz}), 132.2(\mathrm{~s}), 129.4(\mathrm{~s}), 128.9(\mathrm{~d}, J=3.8$ $\mathrm{Hz}), 128.5$ (d, $J=8.6 \mathrm{~Hz}), 128.4$ (s), 128.0 (s), 120.3 (q, $J=271.2 \mathrm{~Hz}), 118.0$ (q, $J=$ $285.5 \mathrm{~Hz}), 115.4(\mathrm{~d}, J=22.0 \mathrm{~Hz}), 79.3(\mathrm{~s}), 56.2(\mathrm{~s}) .{ }^{19} \mathbf{F}$ NMR (565 MHz, $\left.\mathbf{C D C l}_{3}\right) \delta$ -64.36 (s, 3F), -65.98 (s, 3F), -113.00 (s, 1F). HRMS (ESI-TOF) $m / z:[\mathrm{M}+\mathrm{H}]$ calcd for $\mathrm{C}_{18} \mathrm{H}_{13} \mathrm{~N}_{3} \mathrm{~F}_{7}$ 404.0998, found 404.0993.

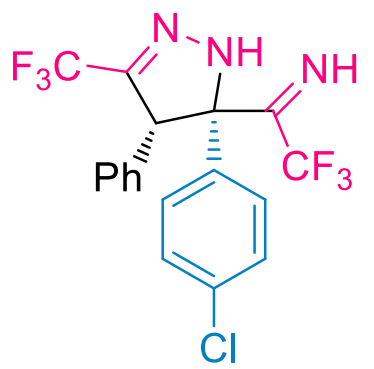

1-(5-(4-Chlorophenyl)-4-phenyl-3-(trifluoromethyl)-4,5-di hydro-1H-pyrazol-5-yl)-2,2,2-trifluoroethanimine $\quad$ (2f): white solid; $60.4 \mathrm{mg} ; 72 \%$ yield; m.p. $111.0-112{ }^{\circ} \mathrm{C} ; \mathrm{R}_{\mathrm{f}}=0.45$ $(\mathrm{PE} / \mathrm{EA}=10 / 1) ;{ }^{1} \mathbf{H}$ NMR (600 MHz, $\left.\mathbf{C D C l}_{3}\right) \delta 11.15$ (s, 1H), $7.08-7.04(\mathrm{~m}, 5 \mathrm{H}), 7.02(\mathrm{~d}, J=8.6 \mathrm{~Hz}, 2 \mathrm{H}), 6.85(\mathrm{dd}, J=6.2$, $2.7 \mathrm{~Hz}, 2 \mathrm{H}), 6.65$ (s, 1H), $5.50(\mathrm{~s}, 1 \mathrm{H}) .{ }^{13} \mathbf{C}$ NMR (151 MHz, CDCl$) \delta 165.7$ (q, $J=$ $29.1 \mathrm{~Hz}), 146.2$ (q, J = 37.2 Hz), 134.4 (s), 132.0 (s), 131.8 (s), 129.4 (s), 128.6 (s), 128.4 (s), 128.1 (s), 128.0 (s), 120.3 (q, $J=271.1 \mathrm{~Hz}), 118.0$ (q, $J=285.3 \mathrm{~Hz}), 79.3$ (s), 56.2 (s). ${ }^{19}$ F NMR (565 MHz, CDCl $) \delta-64.36$ (s, 3F), -65.97 (s, 3F). HRMS (ESI-TOF) $\boldsymbol{m} / \boldsymbol{z}:[\mathrm{M}+\mathrm{H}]$ calcd for $\mathrm{C}_{18} \mathrm{H}_{13} \mathrm{~N}_{3} \mathrm{~F}_{6} \mathrm{Cl} 420.0702$, found 420.0697 .

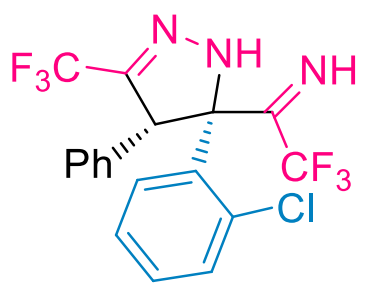

1-(5-(2-chlorophenyl)-4-phenyl-3-(trifluoromethyl)-4,5-dih ydro-1H-pyrazol-5-yl)-2,2,2-trifluoroethanimine (2g): white solid; $71.4 \mathrm{mg} ; 85 \%$ yield; m.p. $126-127{ }^{\circ} \mathrm{C} ; \mathrm{R}_{\mathrm{f}}=0.45$ 
$(\mathrm{PE} / \mathrm{EA}=10 / 1) ;{ }^{1} \mathbf{H}$ NMR (600 MHz, $\left.\mathbf{C D C l}_{3}\right) \delta 10.89(\mathrm{~s}, 1 \mathrm{H}), 7.75-7.70(\mathrm{~m}, 1 \mathrm{H})$, $7.17-6.95(\mathrm{~m}, 8 \mathrm{H}), 6.58(\mathrm{~s}, 1 \mathrm{H}), 5.64(\mathrm{~s}, 1 \mathrm{H}),{ }^{13} \mathbf{C}$ NMR (151 MHz, CDCl 3$) \delta 162.4$ (q, $J=28.7 \mathrm{~Hz}), 148.7$ (q, $J=36.6 \mathrm{~Hz}), 133.0$ (s), 132.4 (s), 131.9 (s), 130.3 (s), 130.1 (s), 129.4 (s), 129.3 (s), 129.2 (s), 128.1 (s), 126.8 (s), 120.3 (q, J = 271.5 Hz),

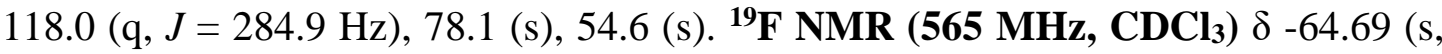
3F), $-67.38(\mathrm{~s}, 3 \mathrm{~F})$. HRMS (ESI-TOF) $\mathrm{m} / \mathrm{z}:[\mathrm{M}+\mathrm{H}]$ calcd for $\mathrm{C}_{18} \mathrm{H}_{13} \mathrm{~N}_{3} \mathrm{~F}_{6} \mathrm{Cl}$ 420.0702, found 420.0706 .

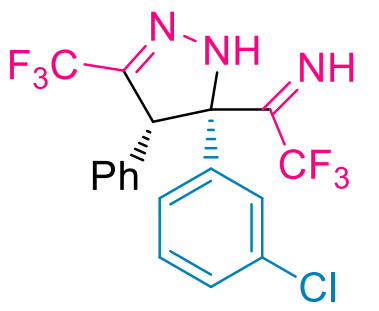

\section{1-(5-(3-Chlorophenyl)-4-phenyl-3-(trifluoromethyl)-4,5-di} hydro-1H-pyrazol-5-yl)-2,2,2-trifluoroethanimine $\quad(2 \mathrm{~h})$ : white solid; $73.9 \mathrm{mg} ; 88 \%$ yield; m.p. $115-116{ }^{\circ} \mathrm{C} ; \mathrm{R}_{\mathrm{f}}=0.45$ $(\mathrm{PE} / \mathrm{EA}=10 / 1) ;{ }^{1} \mathbf{H}$ NMR (600 MHz, $\left.\mathbf{C D C l}_{3}\right) \delta 11.18(\mathrm{~s}, 1 \mathrm{H})$, $7.10(\mathrm{~d}, J=1.8 \mathrm{~Hz}, 1 \mathrm{H}), 7.08-7.04(\mathrm{~m}, 4 \mathrm{H}), 7.02(\mathrm{t}, J=7.8 \mathrm{~Hz}, 1 \mathrm{H}), 6.95(\mathrm{dt}, J=$ 7.5, 1.4 Hz, 1H), $6.85(\mathrm{dd}, J=6.7,2.5 \mathrm{~Hz}, 2 \mathrm{H}), 6.66(\mathrm{~s}, 1 \mathrm{H}), 5.51(\mathrm{~s}, 1 \mathrm{H}) .{ }^{13} \mathbf{C}$ NMR $\left(\mathbf{1 5 1} \mathbf{M H z}, \mathbf{C D C l}_{3}\right) \delta 165.6(\mathrm{q}, J=29.1 \mathrm{~Hz}), 146.1(\mathrm{q}, J=37.0 \mathrm{~Hz}), 135.3(\mathrm{~s}), 134.5$ (s), 131.9 (s), 129.6 (s), 129.4 (s), 128.5 (s), 128.4 (s), 128.1 (s), 126.9 (s), 124.8 (s), $120.3(\mathrm{q}, J=271.1 \mathrm{~Hz}), 117.9$ (q, $J=285.2 \mathrm{~Hz}), 79.3$ (s), 56.4 (s). ${ }^{19}$ F NMR (565 MHz, CDCl 3 ) $\delta-64.34$ (s, 3F), -65.98 (s, 3F). HRMS (ESI-TOF) $\boldsymbol{m} / \boldsymbol{z}:[\mathrm{M}+\mathrm{H}] \mathrm{calcd}$ for $\mathrm{C}_{18} \mathrm{H}_{13} \mathrm{~N}_{3} \mathrm{~F}_{6} \mathrm{Cl} 420.0702$, found 420.0702 .

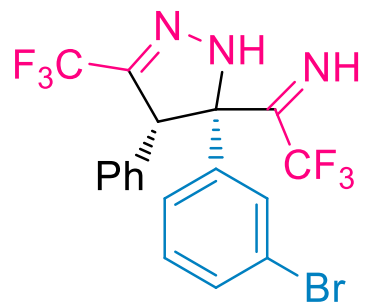

\section{1-(5-(3-Bromophenyl)-4-phenyl-3-(trifluoromethyl)-4,5-di} hydro-1H-pyrazol-5-yl)-2,2,2-trifluoroethanimine (2i): white solid; $73.3 \mathrm{mg} ; 79 \%$ yield; m.p. $81-82{ }^{\circ} \mathrm{C} ; \mathrm{R}_{\mathrm{f}}=0.45$ $(\mathrm{PE} / \mathrm{EA}=10 / 1) ;{ }^{1} \mathbf{H}$ NMR (600 MHz, $\left.\mathbf{C D C l}_{3}\right) \delta 11.19(\mathrm{~s}, 1 \mathrm{H})$, $7.25(\mathrm{~d}, J=1.6 \mathrm{~Hz}, 1 \mathrm{H}), 7.22-7.17(\mathrm{~m}, 1 \mathrm{H}), 7.10-7.05(\mathrm{~m}, 3 \mathrm{H}), 6.99$ (dt, $J=7.9$, $1.3 \mathrm{~Hz}, 1 \mathrm{H}), 6.95$ (t, $J=7.8 \mathrm{~Hz}, 1 \mathrm{H}), 6.85$ (dd, $J=7.0,2.4 \mathrm{~Hz}, 2 \mathrm{H}), 6.66$ (s, 1H), 5.51 (s, 1H). ${ }^{13} \mathbf{C}$ NMR (151 MHz, CDCl $) \delta 165.5$ (q, $\left.J=29.2 \mathrm{~Hz}\right), 146.0$ (q, $\left.J=37.0 \mathrm{~Hz}\right)$, 135.5 (s), 131.9 (s), 131.5 (s), 129.8 (s), 129.7 (s), 129.4 (s), 128.4 (s), 128.1 (s), 125.2 (s), 122.6 (s), 120.3 (q, $J=271.2 \mathrm{~Hz}), 117.9$ (q, $J=285.2 \mathrm{~Hz}), 79.2(\mathrm{~s}), 56.4(\mathrm{~s})$. 
${ }^{19}$ F NMR (565 MHz, CDCl $) \delta-64.33$ (s, 3F), -66.02 (s, 3F). HRMS (ESI-TOF) $m / z:[\mathrm{M}+\mathrm{H}]$ calcd for $\mathrm{C}_{18} \mathrm{H}_{13} \mathrm{~N}_{3} \mathrm{~F}_{6} \mathrm{Br}$ 464.0197, found 464.0197.

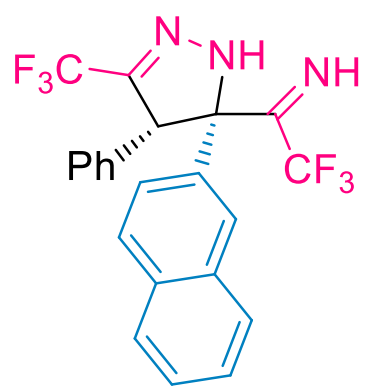

2,2,2-Trifluoro-1-(5-(naphthalen-2-yl)-4-phenyl-3-(trifluor omethyl)-4,5-dihydro-1H-pyrazol-5-yl)ethanimine $\quad(2 \mathrm{j})$ : white solid; $74.8 \mathrm{mg} ; 86 \%$ yield; m.p. $135-136 .{ }^{\circ} \mathrm{C} ; \mathrm{R}_{\mathrm{f}}=0.50$ $(\mathrm{PE} / \mathrm{EA}=10 / 1) ;{ }^{1} \mathbf{H}$ NMR (600 MHz, CDCl$) \delta 11.18$ (s, 1H), 7.69 (ddd, $J=12.2,9.2,2.5 \mathrm{~Hz}, 3 \mathrm{H}), 7.55(\mathrm{~d}, J=8.7 \mathrm{~Hz}, 1 \mathrm{H})$, $7.47-7.39$ (m, 2H), $7.08(\mathrm{dd}, J=8.6,1.9 \mathrm{~Hz}, 1 \mathrm{H}), 6.96-6.87(\mathrm{~m}, 5 \mathrm{H}), 6.85(\mathrm{~s}, 1 \mathrm{H})$, 5.65 (s, 1H). ${ }^{13} \mathbf{C}$ NMR (151 MHz, $\left.\mathbf{C D C l}_{3}\right) \delta 166.0(\mathrm{q}, J=29.0 \mathrm{~Hz}), 146.2(\mathrm{q}, J=$ $36.8 \mathrm{~Hz}$ ), 132.8 (s), 132.7 (s), 132.3 (s), 130.6 (s), 129.5 (s), 128.2 (s), 128.1 (s), 128.1 (s), 127.9 (s), 127.6 (s), 126.7 (s), 126.7 (s), 126.2 (s), 124.0 (s), 120.5 (q, $J=$ $271.1 \mathrm{~Hz}), 118.1$ (q, $J=285.3 \mathrm{~Hz}), 80.0$ (s), 56.2 (s). ${ }^{19} \mathbf{F}$ NMR (565 MHz, CDCl $) \delta$ $-64.20(\mathrm{~s}, 3 \mathrm{~F}),-66.02$ (s, 3F). HRMS (ESI-TOF) $m / z:[\mathrm{M}+\mathrm{H}]$ calcd for $\mathrm{C}_{22} \mathrm{H}_{16} \mathrm{~N}_{3} \mathrm{~F}_{6}$ 436.1248, found 436.1248 .

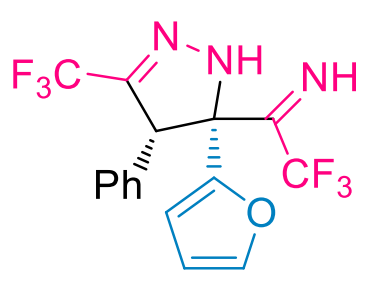

2,2,2-Trifluoro-1-(5-(furan-2-yl)-4-phenyl-3-(trifluorometh yl)-4,5-dihydro-1H-pyrazol-5-yl)ethanimine $\quad(2 \mathrm{k})$ : white solid; $60.0 \mathrm{mg} ; 80 \%$ yield; m.p. $74-75{ }^{\circ} \mathrm{C} ; \mathrm{R}_{\mathrm{f}}=0.47(\mathrm{PE} / \mathrm{EA}=$ 10/1); ${ }^{1} \mathbf{H}$ NMR (600 MHz, $\left.\mathbf{C D C l}_{3}\right) \delta 11.13$ (s, 1H), 7.16 (dd, $J=5.1,1.6 \mathrm{~Hz}, 3 \mathrm{H}), 7.12(\mathrm{~s}, 1 \mathrm{H}), 7.03-6.95(\mathrm{~m}, 2 \mathrm{H}), 6.76(\mathrm{~s}, 1 \mathrm{H}), 6.07$ (dd, $J=3.2$, $1.7 \mathrm{~Hz}, 1 \mathrm{H}), 6.02(\mathrm{~d}, J=3.3 \mathrm{~Hz}, 1 \mathrm{H}), 5.50(\mathrm{~s}, 1 \mathrm{H}) .{ }^{13} \mathbf{C}$ NMR (151 MHz, CDCl $) \delta$ 164.9 (q, $J=30.0 \mathrm{~Hz}), 146.7$ (s), 145.3 (q, $J=36.8 \mathrm{~Hz}), 143.4$ (s), 132.2 (s), 129.4 (s), 129.2 (s), 128.8 (s), 128.3 (s), 128.1 (s), 120.2 (q, $J=271.4 \mathrm{~Hz}), 117.9$ (q, $J=284.4$ Hz), 76.4 (s), 56.0 (s). ${ }^{19}$ F NMR (565 MHz, CDCl $) \delta-64.00$ (s, 3F), -68.04 (s, 3F). HRMS (ESI-TOF) $m / z:[\mathrm{M}+\mathrm{H}]$ calcd for $\mathrm{C}_{16} \mathrm{H}_{12} \mathrm{~N}_{3} \mathrm{OF}_{6} 376.0885$, found 376.0883.

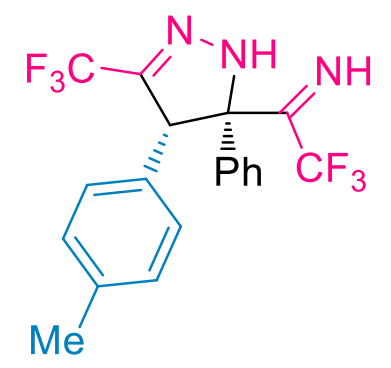

2,2,2-Trifluoro-1-(5-phenyl-4-(p-tolyl)-3-(trifluoromethyl)4,5-dihydro-1H-pyrazol-5-yl)ethanimine (2l): white solid; 
$65.4 \mathrm{mg} ; 82 \%$ yield; m.p. $128-130{ }^{\circ} \mathrm{C} ; \mathrm{R}_{\mathrm{f}}=0.50(\mathrm{PE} / \mathrm{EA}=10 / 1) ;{ }^{1} \mathrm{H}$ NMR $(600$ MHz, $\left.\mathbf{C D C l}_{3}\right) \delta 11.08(\mathrm{~s}, 1 \mathrm{H}), 7.18-6.99(\mathrm{~m}, 5 \mathrm{H}), 6.81(\mathrm{~d}, J=8.7 \mathrm{~Hz}, 2 \mathrm{H}), 6.73(\mathrm{~d}$, $J=8.5 \mathrm{~Hz}, 2 \mathrm{H}), 6.64(\mathrm{~s}, 1 \mathrm{H}), 5.48(\mathrm{~s}, 1 \mathrm{H}), 2.14(\mathrm{~s}, 3 \mathrm{H}) .{ }^{13} \mathbf{C}$ NMR (151 MHz, CDCl $\left.\mathbf{3}\right)$ $\delta 166.1(\mathrm{q}, J=29.0 \mathrm{~Hz}), 146.1(\mathrm{q}, J=36.6 \mathrm{~Hz}), 137.4(\mathrm{~s}), 133.2(\mathrm{~s}), 129.4(\mathrm{~s}), 129.3$ (s), 128.0 (s), 128.3 (s), 128.2 (s), 126.6 (s), 120.5 (q, $J=271.2 \mathrm{~Hz}), 118.1$ (q, $J=$ $285.3 \mathrm{~Hz}$ ), 79.8 (s), 55.9 (s), 21.1 (s). ${ }^{19}$ F NMR (565 MHz, CDCl $) \delta$ ) -64.22 (s, 3F), -66.03 (s, 3F). HRMS (ESI-TOF) $m / z:[\mathrm{M}+\mathrm{H}]$ calcd for $\mathrm{C}_{19} \mathrm{H}_{16} \mathrm{~N}_{3} \mathrm{~F}_{6} 400.1248$, found 400.1252 .

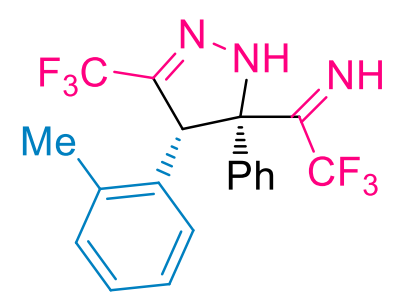

2,2,2-Trifluoro-1-(5-phenyl-4-(o-tolyl)-3-(trifluoromethyl) -4,5-dihydro-1H-pyrazol-5-yl)ethanimine $\quad(2 \mathrm{~m}):$ white solid; $68.6 \mathrm{mg} ; 86 \%$ yield; m.p. $125-127{ }^{\circ} \mathrm{C} ; \mathrm{R}_{\mathrm{f}}=0.50$ $(\mathrm{PE} / \mathrm{EA}=10 / 1) ;{ }^{\mathbf{1}} \mathbf{H}$ NMR (600 MHz, $\left.\mathbf{C D C l}_{3}\right) \delta 11.17(\mathrm{~s}$, 1H), $7.17-7.12(\mathrm{~m}, 2 \mathrm{H}), 7.10-7.05(\mathrm{~m}, 3 \mathrm{H}), 6.93-6.89(\mathrm{~m}, 2 \mathrm{H}), 6.88-6.80(\mathrm{~m}$, 2H), 6.67 (s, 1H), 5.92 (s, 1H), 2.25 (s, 3H). ${ }^{13} \mathbf{C ~ N M R ~ ( 1 5 1 ~ M H z , ~ C D C l ~} \mathbf{~}$ ) $\delta 166.2$ (q, $J=28.8 \mathrm{~Hz}$ ), 146.5 (q, $J=36.7 \mathrm{~Hz}), 136.3$ (s), 133.0 (s), 130.8 (s), 130.3 (s), 129.5 (s), 128.4 (s), 128.1 (s), 127.8 (s), 126.5 (s), 125.9 (s), 120.4 (q, J=271.0 Hz), 118.1 (q, $J=285.2 \mathrm{~Hz}), 79.5$ (s), 51.2 (s), 20.0 (s). ${ }^{19}$ F NMR (565 MHz, CDCl $) \delta-64.41$ (s, 3F), -65.81 (s, 3F). HRMS (ESI-TOF) $\boldsymbol{m} / z:[\mathrm{M}+\mathrm{H}]$ calcd for $\mathrm{C}_{19} \mathrm{H}_{16} \mathrm{~N}_{3} \mathrm{~F}_{6} 400.1248$, found 400.1251 .

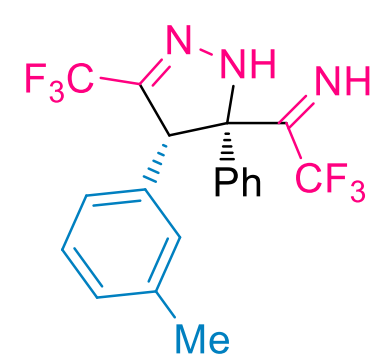

\section{2,2,2-Trifluoro-1-(5-phenyl-4-(m-tolyl)-3-(trifluoromethyl)}

-4,5-dihydro-1H-pyrazol-5-yl)ethanimine (2n): white solid; $59.9 \mathrm{mg} ; 75 \%$ yield; m.p. $122-123{ }^{\circ} \mathrm{C} ; \mathrm{R}_{\mathrm{f}}=0.50(\mathrm{PE} / \mathrm{EA}=$ 10/1); ${ }^{1} \mathbf{H}$ NMR (600 MHz, $\left.\mathbf{C D C l}_{3}\right) \delta 11.09$ (s, 1H), $7.15-$ $7.00(\mathrm{~m}, 5 \mathrm{H}), 6.90(\mathrm{t}, J=7.6 \mathrm{~Hz}, 1 \mathrm{H}), 6.81(\mathrm{~d}, J=7.6 \mathrm{~Hz}, 1 \mathrm{H})$, $6.64(\mathrm{dd}, J=12.6,8.3 \mathrm{~Hz}, 3 \mathrm{H}), 5.47(\mathrm{~s}, 1 \mathrm{H}), 2.11(\mathrm{~s}, 3 \mathrm{H}) .{ }^{13} \mathrm{C}$ NMR (151 MHz, $\left.\mathbf{C D C l}_{3}\right) \delta 166.1(\mathrm{q}, J=29.2 \mathrm{~Hz}), 145.9(\mathrm{q}, J=36.5 \mathrm{~Hz}), 137.7(\mathrm{~s}), 133.1(\mathrm{~s}), 132.2(\mathrm{~s})$, 130.3 (s), 128.4 (s), 128.3 (s), 128.2 (s), 128.0 (s), 126.6 (s), 126.5 (s), 120.4 (q, J = 
$272.1 \mathrm{~Hz}), 118.1$ (q, $J$ = 285.2 Hz), 79.9 (s), 56.1 (s), 21.2 (s). ${ }^{19}$ F NMR (565 MHz, $\left.\mathbf{C D C l}_{3}\right) \delta-64.23(\mathrm{~s}, 3 \mathrm{~F}),-66.00(\mathrm{~s}, 3 \mathrm{~F})$. HRMS (ESI-TOF) $\mathbf{m} / z:[\mathrm{M}+\mathrm{H}]$ calcd for $\mathrm{C}_{19} \mathrm{H}_{16} \mathrm{~N}_{3} \mathrm{~F}_{6} 400.1248$, found 400.1246.

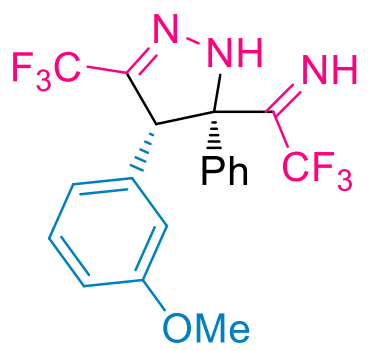

2,2,2-Trifluoro-1-(4-(3-methoxyphenyl)-5-phenyl-3-(trifluo romethyl)-4,5-dihydro-1H-pyrazol-5-yl)ethanimine (20): white solid; $78.0 \mathrm{mg}$; $94 \%$ yield; m.p. $124-125^{\circ} \mathrm{C} ; \mathrm{R}_{\mathrm{f}}=0.35$ $(\mathrm{PE} / \mathrm{EA}=10 / 1) ;{ }^{\mathbf{1}} \mathbf{H}$ NMR (600 MHz, $\left.\mathbf{C D C l}_{3}\right) \delta 11.11(\mathrm{~s}, 1 \mathrm{H})$, $7.13-7.04(\mathrm{~m}, 5 \mathrm{H}), 6.94(\mathrm{t}, J=7.9 \mathrm{~Hz}, 1 \mathrm{H}), 6.70(\mathrm{~s}, 1 \mathrm{H})$, $6.56(\mathrm{~d}, J=7.9 \mathrm{~Hz}, 1 \mathrm{H}), 6.49$ (d, $J=7.4 \mathrm{~Hz}, 1 \mathrm{H}), 6.39$ (s, 1H), 5.50 (s, 1H), 3.61 (s,

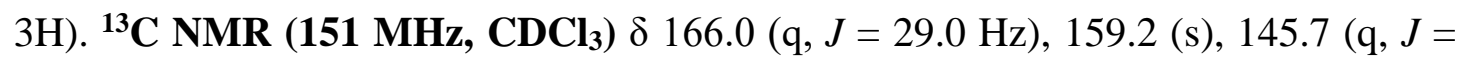
$36.9 \mathrm{~Hz}$ ), 133.8 (s), 133.0 (s), 129.1 (s), 128.4 (s), 128.4 (s), 126.6 (s), 122.1 (s), 120.4 (q, $J=271.2 \mathrm{~Hz}), 118.0$ (q, $J=285.1 \mathrm{~Hz}), 115.0$ (s), 113.6 (s), 79.9 (s), 56.1 (s), 55.2 (s). ${ }^{19}$ F NMR (565 MHz, CDCl 3 ) $\delta$-64.18 (s, 3F), -66.02 (s, 3F). HRMS (ESI-TOF) $m / z:[\mathrm{M}+\mathrm{H}]$ calcd for $\mathrm{C}_{19} \mathrm{H}_{16} \mathrm{~N}_{3} \mathrm{OF}_{6} 416.1198$, found 416.1202 .

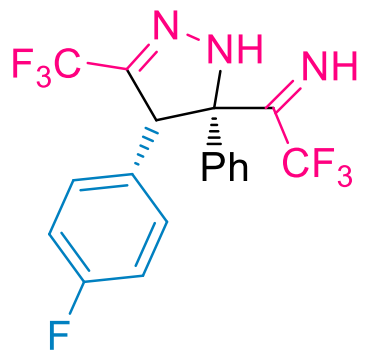

2,2,2-Trifluoro-1-(4-(4-fluorophenyl)-5-phenyl-3-(trifluoro methyl)-4,5-dihydro-1H-pyrazol-5-yl)ethanimine (2p): white solid; $76.6 \mathrm{mg} ; 95 \%$ yield; m.p. $79-81{ }^{\circ} \mathrm{C} ; \mathrm{R}_{\mathrm{f}}=0.49$ $(\mathrm{PE} / \mathrm{EA}=10 / 1) ;{ }^{1} \mathbf{H}$ NMR (600 MHz, $\left.\mathbf{C D C l}_{3}\right) \delta 11.11(\mathrm{~s}, 1 \mathrm{H})$, $7.15-7.08(\mathrm{~m}, 3 \mathrm{H}), 7.07-7.01(\mathrm{~m}, 2 \mathrm{H}), 6.86-6.79(\mathrm{~m}, 2 \mathrm{H})$,

$6.70(\mathrm{dd}, J=16.5,7.7 \mathrm{~Hz}, 3 \mathrm{H}), 5.52(\mathrm{~s}, 1 \mathrm{H}) .{ }^{\mathbf{1 3}} \mathbf{C} \mathbf{N M R}\left(\mathbf{1 5 1} \mathbf{M H z}, \mathbf{C D C l}_{3}\right) \delta 165.9(\mathrm{q}$, $J=29.1 \mathrm{~Hz}), 162.2(\mathrm{~d}, J=246.9 \mathrm{~Hz}), 145.7(\mathrm{q}, J=36.7 \mathrm{~Hz}), 132.9(\mathrm{~s}), 131.2(\mathrm{~d}, J=$ $9.5 \mathrm{~Hz}), 128.5$ (s), 128.2 (d, $J=3.8 \mathrm{~Hz}), 126.5$ (s), 120.4 (q, $J=271.2 \mathrm{~Hz}), 118.0$ (q, $J$ $=285.2 \mathrm{~Hz}), 115.2$ (s), 115.1 (s), 79.8 (s), 55.4 (s). ${ }^{19}$ F NMR (565 MHz, $\left.\mathbf{C D C l}_{3}\right) \delta$ -64.22 (s, 3F), -66.09 (s, 3F), -113.95 (s, 1F). HRMS (ESI-TOF) $m / z:[\mathrm{M}+\mathrm{H}] \mathrm{calcd}$ for $\mathrm{C}_{18} \mathrm{H}_{13} \mathrm{~N}_{3} \mathrm{~F}_{7} 404.0998$, found 404.1001. 


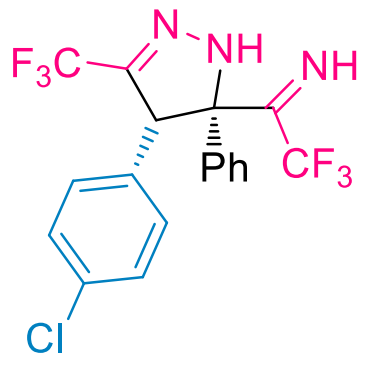

1-(4-(4-Chlorophenyl)-5-phenyl-3-(trifluoromethyl)-4,5-di hydro-1H-pyrazol-5-yl)-2,2,2-trifluoroethanimine (2q): white solid; $66.3 \mathrm{mg} ; 79 \%$ yield; m.p. $111-112{ }^{\circ} \mathrm{C} ; \mathrm{R}_{\mathrm{f}}=0.48$ $(\mathrm{PE} / \mathrm{EA}=10 / 1) ;{ }^{\mathbf{1}} \mathbf{H}$ NMR (600 MHz, CDCl$) \delta 11.11(\mathrm{~s}, 1 \mathrm{H})$, $7.16-7.10(\mathrm{~m}, 3 \mathrm{H}), 7.07-7.03(\mathrm{~m}, 2 \mathrm{H}), 7.00(\mathrm{~d}, J=8.7 \mathrm{~Hz}$, 2H), $6.79(\mathrm{~d}, J=8.3 \mathrm{~Hz}, 2 \mathrm{H}), 6.69(\mathrm{~s}, 1 \mathrm{H}), 5.50(\mathrm{~s}, 1 \mathrm{H}) .{ }^{13} \mathbf{C} \mathbf{N M R}\left(151 \mathrm{MHz}, \mathbf{C D C l}_{3}\right)$ $\delta 165.8(\mathrm{q}, J=29.1 \mathrm{~Hz}), 145.5(\mathrm{q}, J=37.0 \mathrm{~Hz}), 133.8(\mathrm{~s}), 132.7(\mathrm{~s}), 131.0(\mathrm{~s}), 130.8$ (s), 128.7 (s), 128.6 (s), 128.4 (s), 126.4 (s), 120.3 (q, $J=271.0 \mathrm{~Hz}), 118.0$ (q, $J=$ $285.3 \mathrm{~Hz}), 79.9$ (s), 55.4 (s). ${ }^{19} \mathbf{F}$ NMR (565 MHz, CDCl $) \delta-64.22(\mathrm{~s}, 3 \mathrm{~F}),-66.06(\mathrm{~s}$, 3F). HRMS (ESI-TOF) $m / z$ : [M+H] calcd for $\mathrm{C}_{18} \mathrm{H}_{13} \mathrm{~N}_{3} \mathrm{~F}_{6} \mathrm{Cl}$ 420.0702, found 420.0701 .

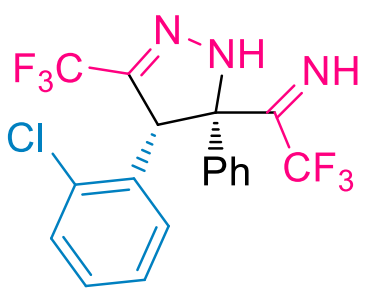

1-(4-(2-Chlorophenyl)-5-phenyl-3-(trifluoromethyl)-4,5-di hydro-1H-pyrazol-5-yl)-2,2,2-trifluoroethanimine (2r): white solid; $73.0 \mathrm{mg} ; 87 \%$ yield; m.p. $104-105{ }^{\circ} \mathrm{C} ; \mathrm{R}_{\mathrm{f}}=0.48$ $(\mathrm{PE} / \mathrm{EA}=10 / 1) ;{ }^{1} \mathbf{H}$ NMR $\left(\mathbf{6 0 0} \mathbf{M H z}, \mathbf{C D C l}_{3}\right) \delta 11.16(\mathrm{~s}, 1 \mathrm{H})$, $7.23(\mathrm{dt}, J=4.4,2.4 \mathrm{~Hz}, 2 \mathrm{H}), 7.12-7.08(\mathrm{~m}, 3 \mathrm{H}), 7.07(\mathrm{dd}, J=7.8,1.4 \mathrm{~Hz}, 1 \mathrm{H}), 7.02$ - $6.95(\mathrm{~m}, 2 \mathrm{H}), 6.95-6.91(\mathrm{~m}, 1 \mathrm{H}), 6.71(\mathrm{~s}, 1 \mathrm{H}), 6.31(\mathrm{~s}, 1 \mathrm{H}) .{ }^{13} \mathbf{C}$ NMR (151 MHz, $\left.\mathbf{C D C l}_{3}\right) \delta 165.1(\mathrm{q}, J=28.9 \mathrm{~Hz}), 146.1(\mathrm{q}, J=37.1 \mathrm{~Hz}), 134.1(\mathrm{~s}), 132.4(\mathrm{~s}), 130.7(\mathrm{~s})$, 130.6 (s), 129.4 (s), 129.3 (s), 128.6 (s), 128.2 (s), 126.8 (s), 126.6 (s), 120.3 (q, $J=$ $271.0 \mathrm{~Hz}), 118.1$ (q, $J=285.3 \mathrm{~Hz}), 79.4(\mathrm{~s}), 51.2(\mathrm{~s}) .{ }^{19} \mathbf{F}$ NMR (565 MHz, $\left.\mathbf{C D C l}_{3}\right) \delta$ -64.61 (s, 3F), -65.67 (s, 3F). HRMS (ESI-TOF) $m / z:[\mathrm{M}+\mathrm{H}]$ calcd for $\mathrm{C}_{18} \mathrm{H}_{13} \mathrm{~N}_{3} \mathrm{~F}_{6} \mathrm{Cl} 420.0702$, found 420.0700 .

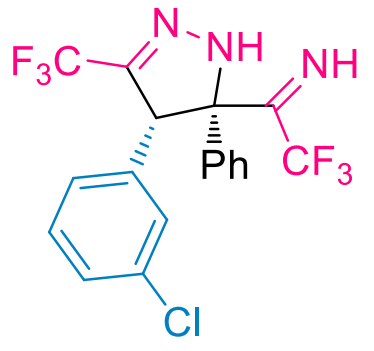

1-(4-(3-Chlorophenyl)-5-phenyl-3-(trifluoromethyl)-4,5-di hydro-1H-pyrazol-5-yl)-2,2,2-trifluoroethanimine (2s): white solid; $65.5 \mathrm{mg} ; 78 \%$ yield; m.p. $82-83{ }^{\circ} \mathrm{C} ; \mathrm{R}_{\mathrm{f}}=0.48$ $(\mathrm{PE} / \mathrm{EA}=10 / 1)$; ${ }^{\mathbf{1}} \mathrm{H}$ NMR (600 MHz, $\left.\mathbf{C D C l}_{3}\right) \delta 11.12$ (s, 1H), $7.16-7.11(\mathrm{~m}, 3 \mathrm{H}), 7.09-7.04(\mathrm{~m}, 2 \mathrm{H}), 7.02-6.98(\mathrm{~m}, 1 \mathrm{H})$, 
$6.94(\mathrm{t}, J=7.8 \mathrm{~Hz}, 1 \mathrm{H}), 6.86(\mathrm{~s}, 1 \mathrm{H}), 6.74(\mathrm{~d}, J=7.6 \mathrm{~Hz}, 1 \mathrm{H}), 6.70(\mathrm{~s}, 1 \mathrm{H}), 5.50(\mathrm{~s}$, 1H). ${ }^{13} \mathbf{C}$ NMR (151 MHz, $\left.\mathbf{C D C l}_{3}\right) \delta 165.7$ (q, $\left.J=29.2 \mathrm{~Hz}\right), 145.1$ (q, $\left.J=37.0 \mathrm{~Hz}\right)$, 134.5 (s), 134.0 (s), 132.6 (s), 129.6 (s), 129.4 (s), 128.7 (s), 128.6 (s), 128.0 (s), 127.6 (s), 126.4 (s), 120.3 (q, $J=271.1 \mathrm{~Hz}), 118.0$ (q, $J=285.2 \mathrm{~Hz}), 80.0$ (s), 55.6 (s). ${ }^{19}$ F NMR (565 MHz, CDCl $) \delta-64.23$ (s, 3F), -66.05 (s, 3F). HRMS (ESI-TOF) $m / z:[\mathrm{M}+\mathrm{H}]$ calcd for $\mathrm{C}_{18} \mathrm{H}_{13} \mathrm{~N}_{3} \mathrm{~F}_{6} \mathrm{Cl} 420.0702$, found 420.0707 .

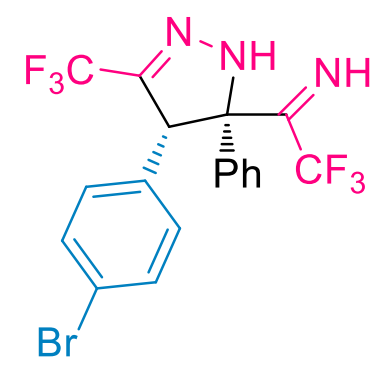

1-(4-(4-Bromophenyl)-5-phenyl-3-(trifluoromethyl)-4,5-di hydro-1H-pyrazol-5-yl)-2,2,2-trifluoroethanimine (2t): white solid; $76.8 \mathrm{mg} ; 83 \%$ yield; m.p. $122-124{ }^{\circ} \mathrm{C} ; \mathrm{R}_{\mathrm{f}}=0.47$ $(\mathrm{PE} / \mathrm{EA}=10 / 1) ;{ }^{\mathbf{1}} \mathbf{H}$ NMR (600 MHz, $\left.\mathbf{C D C l}_{3}\right) \delta 11.11(\mathrm{~s}, 1 \mathrm{H})$, $7.16(\mathrm{~s}, 1 \mathrm{H}), 7.15-7.11(\mathrm{~m}, 4 \mathrm{H}), 7.08-7.01(\mathrm{~m}, 2 \mathrm{H}), 6.77-$ $6.63(\mathrm{~m}, 3 \mathrm{H}), 5.50$ (s, 1H). ${ }^{13} \mathbf{C}$ NMR (151 MHz, $\left.\mathbf{C D C l}_{3}\right) \delta 165.8(\mathrm{q}, J=29.1 \mathrm{~Hz})$, $145.4(\mathrm{q}, J=36.9 \mathrm{~Hz}), 132.7$ (s), 131.6 (s), 131.3 (s), 131.2 (s), 128.7 (s), 128.6 (s), 126.4 (s), 122.0 (s), 120.3 (q, $J=271.2 \mathrm{~Hz}), 118.0$ (q, $J=285.2 \mathrm{~Hz}), 79.8$ (s), 55.5 (s). ${ }^{19}$ F NMR (565 MHz, CDCl $) \delta-64.25$ (s, 3F), -66.08 (s, 3F). HRMS (ESI-TOF) $m / z:[\mathrm{M}+\mathrm{H}]$ calcd for $\mathrm{C}_{18} \mathrm{H}_{13} \mathrm{~N}_{3} \mathrm{BrF}_{6} 464.0197$, found 464.0194 .

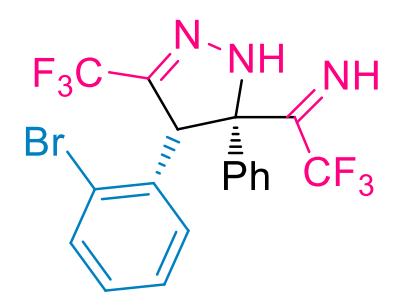

1-(4-(2-Bromophenyl)-5-phenyl-3-(trifluoromethyl)-4,5-di hydro-1H-pyrazol-5-yl)-2,2,2-trifluoroethanimine $\quad(2 \mathrm{u})$ : white solid; $70.4 \mathrm{mg} ; 76 \%$ yield; m.p. $111-112{ }^{\circ} \mathrm{C} ; \mathrm{R}_{\mathrm{f}}=0.47$ $(\mathrm{PE} / \mathrm{EA}=10 / 1) ;{ }^{1} \mathbf{H}$ NMR (600 MHz, $\left.\mathbf{C D C l}_{3}\right) \delta 11.16(\mathrm{~s}$, $1 \mathrm{H}), 7.26(\mathrm{ddd}, J=6.2,4.3,1.3 \mathrm{~Hz}, 3 \mathrm{H}), 7.13-7.07(\mathrm{~m}, 3 \mathrm{H}), 7.03(\mathrm{td}, J=7.7,1.2 \mathrm{~Hz}$, 1H), $6.93(\mathrm{dd}, J=7.8,1.7 \mathrm{~Hz}, 1 \mathrm{H}), 6.90-6.85(\mathrm{~m}, 1 \mathrm{H}), 6.69(\mathrm{~s}, 1 \mathrm{H}), 6.32(\mathrm{~s}, 1 \mathrm{H}) .{ }^{13} \mathrm{C}$ NMR (151 MHz, CDCl 3 ) $\delta 165.0(\mathrm{q}, J=28.9 \mathrm{~Hz}), 146.3(\mathrm{q}, J=37.2 \mathrm{~Hz}), 132.8(\mathrm{~s})$, 132.4 (s), 132.4 (s), 130.8 (s), 129.5 (s), 128.6 (s), 128.2 (s), 127.4 (s), 126.8 (s), $125.0(\mathrm{~s}), 120.2(\mathrm{q}, J=271.2 \mathrm{~Hz}), 118.0(\mathrm{q}, J=285.4 \mathrm{~Hz}), 79.5(\mathrm{~s}), 54.0(\mathrm{~s}) .{ }^{19} \mathbf{F}$ NMR (565 MHz, CDCl ${ }_{3}$ ) $\delta-64.60$ (s, 3F), -65.60 (s, 3F). HRMS (ESI-TOF) $\mathbf{m} / z$ : $[\mathrm{M}+\mathrm{H}]$ calcd for $\mathrm{C}_{18} \mathrm{H}_{13} \mathrm{~N}_{3} \mathrm{BrF}_{6}$ 464.0197, found 464.0200. 


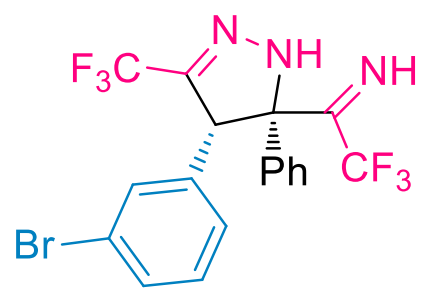

1-(4-(3-Bromophenyl)-5-phenyl-3-(trifluoromethyl)-4,5

-dihydro-1H-pyrazol-5-yl)-2,2,2-trifluoroethanimine

(2v): white solid; $82.4 \mathrm{mg}$; $89 \%$ yield; m.p. $103-104{ }^{\circ} \mathrm{C}$; $\mathrm{R}_{\mathrm{f}}=0.47(\mathrm{PE} / \mathrm{EA}=10 / 1) ;{ }^{1} \mathbf{H}$ NMR $\left(\mathbf{6 0 0} \mathbf{M H z}, \mathbf{C D C l}_{3}\right) \delta$ 11.12 (s, 1H), $7.17-7.09$ (m, 4H), $7.09-7.03(\mathrm{~m}, 2 \mathrm{H}), 7.01$ (s, 1H), 6.89 (t, J = 7.9 Hz, 1H), 6.78 (d, J = 7.5 Hz, 1H), 6.72 (s, 1H), 5.49 (s, 1H). ${ }^{13}$ C NMR (151 MHz, $\left.\mathbf{C D C l}_{3}\right) \delta 165.7(\mathrm{q}, J=29.2 \mathrm{~Hz}), 145.1(\mathrm{q}, J=37.1 \mathrm{~Hz}), 134.8(\mathrm{~s}), 132.6(\mathrm{~s}), 132.5$ (s), 130.9 (s), 129.6 (s), 128.7 (s), 128.5 (s), 128.0 (s), 126.4 (s), 122.1 (s), 120.3 (q, $J=$ $271.1 \mathrm{~Hz}), 118.0$ (q, $J=285.1 \mathrm{~Hz}), 80.1$ (s), $55.6(\mathrm{~s}) .{ }^{19} \mathbf{F}$ NMR (565 MHz, $\left.\mathbf{C D C l}_{3}\right) \delta$ -64.20 (s, 3F), -66.04 (s, 3F). HRMS (ESI-TOF) $\mathrm{m} / \mathrm{z}:[\mathrm{M}+\mathrm{H}]$ calcd for $\mathrm{C}_{18} \mathrm{H}_{13} \mathrm{~N}_{3} \mathrm{BrF}_{6}$ 464.0197, found 464.0197.

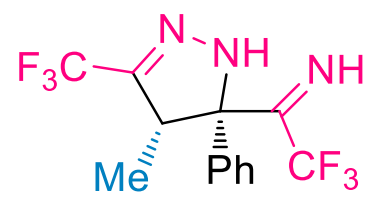

2,2,2-Trifluoro-1-(4-methyl-5-phenyl-3-(trifluoromethyl)-4 ,5-dihydro-1H-pyrazol-5-yl)ethanimine (2w): white solid; $53.0 \mathrm{mg} ; 82 \%$ yield; m.p. $125-126{ }^{\circ} \mathrm{C} ; \mathrm{R}_{\mathrm{f}}=0.52(\mathrm{PE} / \mathrm{EA}=$ 10/1); ${ }^{1} \mathbf{H}$ NMR (600 MHz, $\left.\mathbf{C D C l}_{3}\right) \delta 10.98$ (s, 1H), $7.42-7.38$ (m, 2H), $7.38-7.35$ (m, 1H), $7.29-7.25(\mathrm{~m}, 2 \mathrm{H}), 6.52(\mathrm{~s}, 1 \mathrm{H}), 4.31(\mathrm{q}, J=7.4 \mathrm{~Hz}, 1 \mathrm{H}), 0.73(\mathrm{~d}, J=7.4$

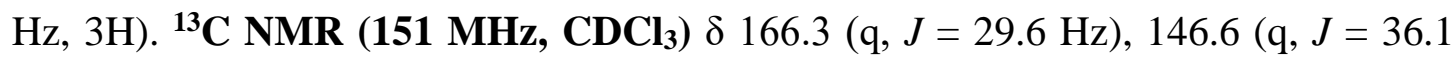
Hz), 133.7 (s), 129.1 (s), 128.9 (s), 126.2 (s), 120.6 (q, $J=270.6 \mathrm{~Hz}), 118.0$ (q, $J=$ 285.3 Hz), 79.3 (s), 44.6 (s), 12.2 (s). ${ }^{19}$ F NMR (565 MHz, CDCl $) \delta-65.04$ (s, 3F), $-66.72(\mathrm{~s}, 3 \mathrm{~F})$. HRMS (ESI-TOF) $m / z:[\mathrm{M}+\mathrm{H}]$ calcd for $\mathrm{C}_{13} \mathrm{H}_{12} \mathrm{~N}_{3} \mathrm{~F}_{6} 324.0935$, found 324.0939 .

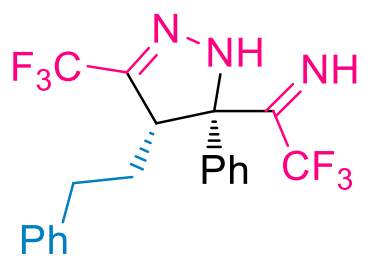

2,2,2-Trifluoro-1-(4-phenethyl-5-phenyl-3-(trifluoromethyl )-4,5-dihydro-1H-pyrazol-5-yl)ethanimine (2x): white solid; $57.8 \mathrm{mg} ; 70 \%$ yield; m.p. $120-121{ }^{\circ} \mathrm{C} ; \mathrm{R}_{\mathrm{f}}=0.51(\mathrm{PE} / \mathrm{EA}=$ 10/1); ${ }^{1} \mathbf{H}$ NMR (600 MHz, CDCl 3$) \delta 10.99$ (s, 1H), $7.49-$ $7.42(\mathrm{~m}, 3 \mathrm{H}), 7.42-7.38(\mathrm{~m}, 2 \mathrm{H}), 7.13(\mathrm{t}, J=7.3 \mathrm{~Hz}, 2 \mathrm{H}), 7.09(\mathrm{dd}, J=8.3,6.3 \mathrm{~Hz}$, $1 \mathrm{H}), 6.69(\mathrm{~d}, J=7.1 \mathrm{~Hz}, 2 \mathrm{H}), 6.61(\mathrm{~s}, 1 \mathrm{H}), 4.43(\mathrm{t}, J=5.0 \mathrm{~Hz}, 1 \mathrm{H}), 2.38(\mathrm{td}, J=12.8$, 
$5.0 \mathrm{~Hz}, 1 \mathrm{H}), 2.18(\mathrm{td}, J=12.8,5.1 \mathrm{~Hz}, 1 \mathrm{H}), 1.67-1.58(\mathrm{~m}, 1 \mathrm{H}), 1.52-1.44(\mathrm{~m}, 1 \mathrm{H})$.

${ }^{13}$ C NMR (151 MHz, CDCl 3$) \delta 166.2(q, J=28.5 \mathrm{~Hz}), 145.9(\mathrm{q}, J=36.5 \mathrm{~Hz}), 141.4$ (s), 133.3 (s), 129.2 (s), 129.1 (s), 128.4 (s), 128.2 (s), 127.0 (s), 126.0 (s), 120.7 (q, $J$ $=270.5 \mathrm{~Hz}), 118.1(\mathrm{q}, J=285.2 \mathrm{~Hz}), 79.0$ (s), 48.8 (s), 31.9 (s), 29.4 (s). ${ }^{19}$ F NMR (565 MHz, CDCl $) \delta-64.61(\mathrm{~s}, 3 \mathrm{~F}),-66.08(\mathrm{~s}, 3 \mathrm{~F})$. HRMS (ESI-TOF) $\mathbf{m} / \boldsymbol{z}:[\mathrm{M}+\mathrm{H}]$ calcd for $\mathrm{C}_{20} \mathrm{H}_{18} \mathrm{~N}_{3} \mathrm{~F}_{6} 414.1405$, found 414.1399.

General procedure for the preparation of 3-trifluoromethyl pyrazoles 5

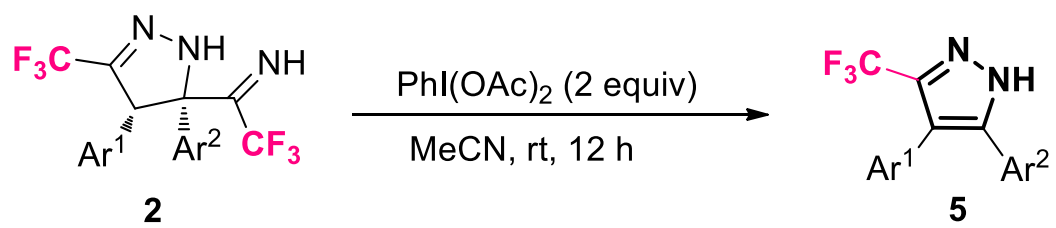

To a Schlenk tube with a magnetic stirring bar, $0.1 \mathrm{mmol}$ of 2, $\mathrm{PhI}(\mathrm{OAc})_{2}(64.4 \mathrm{mg}$, $0.2 \mathrm{mmol}, 2.0$ equiv) and $2 \mathrm{~mL}$ dry $\mathrm{MeCN}$ was added. The mixture was reacted at room temperature for 12 hours. After the reaction was completed (monitored by TLC), the resulting suspension was extracted with $\mathrm{DCM}(3 \times 10 \mathrm{~mL})$, then the organic extracts were combined, dried over $\mathrm{MgSO}_{4}$, filtered and concentrated in vacuo. After being concentrated, the residue was purified by flash column chromatography on silica gel (eluting with petroleum ether/ethyl acetate $=10 / 1$ ) to give the product 5 .<smiles>FC(F)(F)c1n[nH]c(-c2ccccc2)c1-c1ccccc1</smiles>

4,5-Diphenyl-3-(trifluoromethyl)-1H-pyrazole (5a): white solid; $27.6 \mathrm{mg} ; 96 \%$ yield; m.p. $200-201{ }^{\circ} \mathrm{C} ; \mathrm{R}_{\mathrm{f}}=0.17(\mathrm{PE} / \mathrm{EA}=10 / 1) ;{ }^{1} \mathbf{H}$ NMR (600 MHz, CDCl $) \delta 10.99(\mathrm{~s}, 1 \mathrm{H}), 7.37-7.33(\mathrm{~m}, 5 \mathrm{H}), 7.33-$ $7.30(\mathrm{~m}, 1 \mathrm{H}), 7.29$ - $7.25(\mathrm{~m}, 4 \mathrm{H}) .{ }^{13} \mathrm{C}$ NMR (151 MHz, DMSO-d $) \delta 142.1(\mathrm{~s})$, 140.1 (q, $J=35.1 \mathrm{~Hz}), 131.3$ (s), 130.6 (s), 129.3 (s), 129.2 (s), 129.0 (s), 128.6 (s), 128.3 (s), 128.2 (s), 122.4 (q, $J=269.4 \mathrm{~Hz}), 118.0$ (s). ${ }^{19}$ F NMR (565 MHz, DMSO-d $)) \delta-58.05(\mathrm{~s}, 3 \mathrm{~F})$. HRMS (ESI-TOF) $m / z:[\mathrm{M}+\mathrm{H}]$ calcd for $\mathrm{C}_{16} \mathrm{H}_{12} \mathrm{~N}_{2} \mathrm{~F}_{3}$ 289.0953, found 289.0951 . 


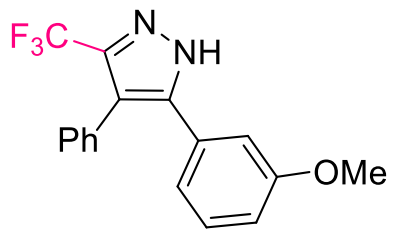

5-(3-Methoxyphenyl)-4-phenyl-3-(trifluoromethyl)-1H-p yrazole (5b): white solid; $30.5 \mathrm{mg}$; 96\% yield; m.p. $171-172{ }^{\circ} \mathrm{C} ; \mathrm{R}_{\mathrm{f}}=0.12(\mathrm{PE} / \mathrm{EA}=10 / 1) ;{ }^{1} \mathbf{H}$ NMR $(\mathbf{6 0 0} \mathrm{MHz}$, CDCl $\left._{3}\right) \delta 7.36(\mathrm{~s}, 3 \mathrm{H}), 7.30-7.18(\mathrm{~m}, 3 \mathrm{H}), 6.89(\mathrm{dd}, J=$ 18.1, $8.7 \mathrm{~Hz}, 2 \mathrm{H}), 6.75(\mathrm{~d}, J=5.6 \mathrm{~Hz}, 1 \mathrm{H}), 3.57$ (d, $J=8.8 \mathrm{~Hz}, 3 \mathrm{H}) .{ }^{13} \mathbf{C}$ NMR $(151$ MHz, $\left.\mathbf{C D C l}_{3}\right) \delta 159.8(\mathrm{~s}), 142.3(\mathrm{~s}), 141.2(\mathrm{q}, J=35.1 \mathrm{~Hz}), 130.6(\mathrm{~s}), 130.4(\mathrm{~s}), 130.2$ (s), $129.3(\mathrm{~s}), 128.6(\mathrm{~s}), 128.0(\mathrm{~s}), 121.4(\mathrm{q}, J=269.8 \mathrm{~Hz}), 119.7(\mathrm{~s}), 118.4(\mathrm{~s}), 115.5$ (s), 112.6 (s), 55.1 (s). ${ }^{19} \mathbf{F}$ NMR (565 MHz, $\left.\mathbf{C D C l}_{3}\right) \delta-59.55$ (s, 3F). HRMS (ESI-TOF) $m / z:[\mathrm{M}+\mathrm{H}]$ calcd for $\mathrm{C}_{17} \mathrm{H}_{14} \mathrm{~N}_{2} \mathrm{OF}_{3} 319.1058$, found 319.1060 .

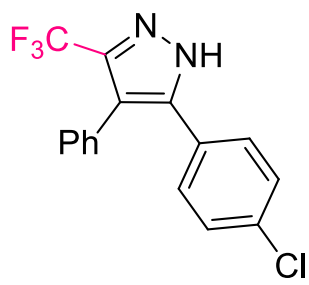

6-(4-Chlorophenyl)-4-phenyl-3-(trifluoromethyl)-1H-pyrazole (5c): white solid; $30.3 \mathrm{mg}$; $94 \%$ yield; m.p. $202-204{ }^{\circ} \mathrm{C} ; \mathrm{R}_{\mathrm{f}}=$ $0.16(\mathrm{PE} / \mathrm{EA}=10 / 1) ;{ }^{1} \mathbf{H}$ NMR $\left(\mathbf{6 0 0} \mathbf{~ M H z}, \mathbf{C D C l}_{3}\right) \delta 7.36(\mathrm{dd}, J$ $\mathrm{Cl}=4.1,1.7 \mathrm{~Hz}, 3 \mathrm{H}), 7.27(\mathrm{dd}, J=8.5,1.7 \mathrm{~Hz}, 2 \mathrm{H}), 7.23(\mathrm{~d}, J=3.5$

$\mathrm{Hz}, 2 \mathrm{H}), 7.19(\mathrm{dd}, J=8.5,1.8 \mathrm{~Hz}, 2 \mathrm{H}) .{ }^{13} \mathbf{C}$ NMR $\left(151 \mathbf{M H z}, \mathbf{C D C l}_{3}\right) \delta 141.7(\mathrm{~s})$, 141.2 (q, $J=36.6 \mathrm{~Hz}), 135.4$ (s), 130.2 (s), 130.0 (s), 129.4 (s), 128.9 (s), 128.7 (s), 128.2 (s), 126.6 (s), 121.3 (q, $J=270.1 \mathrm{~Hz}), 118.7$ (s). ${ }^{19} \mathbf{F}$ NMR (565 $\left.\mathbf{~ M H z}, \mathbf{C D C l}_{3}\right)$ $\delta$-59.59 (s, 3F). HRMS (ESI-TOF) $\mathbf{m} / z$ : [M+H] calcd for $\mathrm{C}_{16} \mathrm{H}_{11} \mathrm{~N}_{2} \mathrm{~F}_{3} \mathrm{Cl} 323.0563$, found 323.0559.

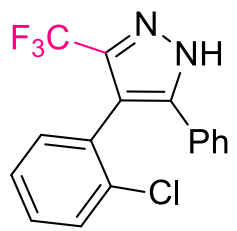

4-(2-Chlorophenyl)-5-phenyl-3-(trifluoromethyl)- $1 H$-pyrazole

(5d): white solid; $28.7 \mathrm{mg} ; 89 \%$ yield; m.p. $147-148{ }^{\circ} \mathrm{C} ; \mathrm{R}_{\mathrm{f}}=0.16$ $(\mathrm{PE} / \mathrm{EA}=10 / 1) ;{ }^{1} \mathbf{H}$ NMR (600 MHz, $\left.\mathbf{C D C l}_{3}\right) \delta \delta 12.38(\mathrm{~s}, 1 \mathrm{H}), 7.45$ (s, 1H), $7.38-7.26(\mathrm{~m}, 8 \mathrm{H}) .{ }^{13} \mathbf{C}$ NMR (151 MHz, $\left.\mathbf{C D C l}_{3}\right) \delta 143.0$ (s), 141.8 (q, $J=36.1 \mathrm{~Hz}), 135.2(\mathrm{~s}), 132.2$ (s), 129.9 (s), $129.8(\mathrm{~s}), 129.7$ (s), 129.3 (s), 129.1 (s), 128.1 (s), 126.8 (s), 126.8 (s), 121.2 (q, $J=270.1 \mathrm{~Hz}), 115.3$ (s). ${ }^{19} \mathbf{F}$ NMR (565 MHz, CDCl 3 ) $\delta$-60.73 (s, 3F). HRMS (ESI-TOF) $\mathrm{m} / \mathrm{z}:[\mathrm{M}+\mathrm{H}]$ calcd for $\mathrm{C}_{16} \mathrm{H}_{11} \mathrm{~N}_{2} \mathrm{~F}_{3} \mathrm{Cl}$ 323.0563, found 323.0560. 


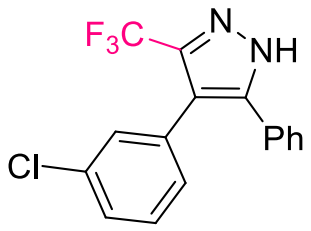

5-(3-Chlorophenyl)-5-phenyl-3-(trifluoromethyl)-1H-pyrazole

(5e): white solid; $29.4 \mathrm{mg} ; 91 \%$ yield; m.p. $150-151{ }^{\circ} \mathrm{C} ; \mathrm{R}_{\mathrm{f}}=$ $0.16(\mathrm{PE} / \mathrm{EA}=10 / 1) ;{ }^{1} \mathbf{H}$ NMR (600 MHz, CDCl 3$) \delta 12.75(\mathrm{~s}$, 1H), $7.38-7.30(\mathrm{~m}, 4 \mathrm{H}), 7.30-7.26(\mathrm{~m}, 3 \mathrm{H}), 7.20$ (s, 1H), 7.09 $(\mathrm{d}, J=7.6 \mathrm{~Hz}, 1 \mathrm{H}) .{ }^{13} \mathbf{C} \mathbf{N M R}\left(\mathbf{1 5 1} \mathbf{M H z}, \mathbf{C D C l}_{3}\right) \delta 142.8(\mathrm{~s}), 141.1(\mathrm{q}, J=36.3 \mathrm{~Hz})$, 134.4 (s), 132.3 (s), $130.3(\mathrm{~s}), 129.9$ (s), 129.5 (s), 129.2 (s), 128.6 (s), 128.3 (s), 127.6 (s), $127.5(\mathrm{~s}), 121.3(\mathrm{q}, J=270.1 \mathrm{~Hz}), 116.8(\mathrm{~s}) .{ }^{19} \mathbf{F}$ NMR (565 MHz, $\left.\mathbf{C D C l}_{3}\right) \delta$ -59.41 (s, 3F). HRMS (ESI-TOF) $m / z$ : [M+H] calcd for $\mathrm{C}_{16} \mathrm{H}_{11} \mathrm{~N}_{2} \mathrm{~F}_{3} \mathrm{Cl} 323.0563$, found 323.0560 .

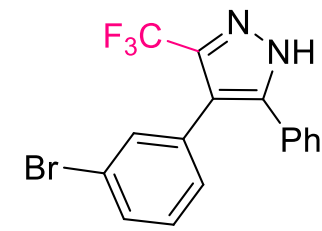

4-(3-Bromophenyl)-5-phenyl-3-(trifluoromethyl)- $1 H$-pyrazole (5f): white solid; $33.0 \mathrm{mg} ; 90 \%$ yield; m.p. $172-173{ }^{\circ} \mathrm{C} ; \mathrm{R}_{\mathrm{f}}=0.14$ $(\mathrm{PE} / \mathrm{EA}=10 / 1) ;{ }^{1} \mathbf{H}$ NMR (600 MHz, $\left.\mathbf{C D C l}_{3}\right) \delta 7.48(\mathrm{dd}, J=8.1$, $0.6 \mathrm{~Hz}, 1 \mathrm{H}), 7.40-7.30(\mathrm{~m}, 4 \mathrm{H}), 7.27-7.23(\mathrm{~m}, 2 \mathrm{H}), 7.21(\mathrm{t}, J$ $=7.8 \mathrm{~Hz}, 1 \mathrm{H}), 7.14(\mathrm{~d}, J=7.6 \mathrm{~Hz}, 1 \mathrm{H}) .{ }^{13} \mathbf{C} \mathbf{N M R}\left(\mathbf{1 5 1} \mathbf{~ M H z}, \mathbf{C D C l}_{3}\right) \delta 142.8(\mathrm{~s})$, 141.2 (q, $J=36.2 \mathrm{~Hz}), 133.1$ (s), 132.6 (s), 131.2 (s), 130.1 (s), 129.5 (s), 129.2 (s), 129.1 (s), 127.6 (s), 127.5 (s), 122.5 (s), 121.3 (q, $J=270.0$ Hz), 116.8 (s). ${ }^{19}$ F NMR $\left(565 \mathrm{MHz}, \mathbf{C D C l}_{3}\right) \delta-59.47(\mathrm{~s}, 3 \mathrm{~F})$. HRMS (ESI-TOF) $\mathrm{m} / \mathrm{z}:[\mathrm{M}+\mathrm{H}]$ calcd for $\mathrm{C}_{16} \mathrm{H}_{11} \mathrm{~N}_{2} \mathrm{BrF}_{3} 367.0058$, found367.0055 .

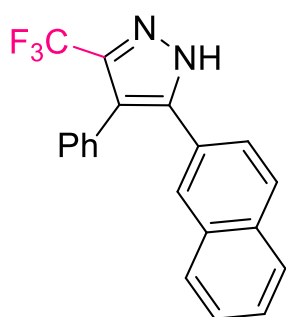

4-(Naphthalen-2-yl)-4-phenyl-3-(trifluoromethyl)- $1 H$-pyrazole (5g): white solid; $31.1 \mathrm{mg} ; 92 \%$ yield; m.p. $197-198{ }^{\circ} \mathrm{C} ; \mathrm{R}_{\mathrm{f}}=0.13$ $(\mathrm{PE} / \mathrm{EA}=10 / 1) ;{ }^{1} \mathbf{H}$ NMR (600 MHz, DMSO-d 6 ) $\delta 7.91$ (s, 1H), $7.83(\mathrm{dd}, J=19.3,8.5 \mathrm{~Hz}, 2 \mathrm{H}), 7.75(\mathrm{~d}, J=7.0 \mathrm{~Hz}, 1 \mathrm{H}), 7.53-$ $7.46(\mathrm{~m}, 2 \mathrm{H}), 7.38-7.32(\mathrm{~m}, 3 \mathrm{H}), 7.30-7.22(\mathrm{~m}, 3 \mathrm{H}) .{ }^{13} \mathbf{C} \mathbf{~ N M R}$ (151 MHz, DMSO-d $) \delta 142.2$ (s), 140.1 (q, $J=34.9$ Hz), 133.1 (s), 131.3 (s), 130.7 (s), 129.4 (s), 129.0 (s), 128.8 (s), 128.5 (s), 128.4 (s), 128.2 (s), 127.6 (s), 127.5 (s), 127.4 (s), 126.1 (s), 125.6 (s), 122.5 (q, $J=269.5 \mathrm{~Hz}), 118.3$ (s). ${ }^{19}$ F NMR (565 MHz, 
DMSO-d $\left.\boldsymbol{d}_{\boldsymbol{\sigma}}\right) \delta-57.96(\mathrm{~s}, 3 \mathrm{~F})$. HRMS (ESI-TOF) $m / z:[\mathrm{M}+\mathrm{H}]$ calcd for $\mathrm{C}_{20} \mathrm{H}_{14} \mathrm{~N}_{2} \mathrm{~F}_{3}$ 339.1109 , found 339.1108.

General procedure for the preparation of 3,5-ditrifluoromethyl pyridazines 6<smiles>N=C([TeH])C1([18F])NN=C(C(F)(F)F)C1[18F]</smiles>

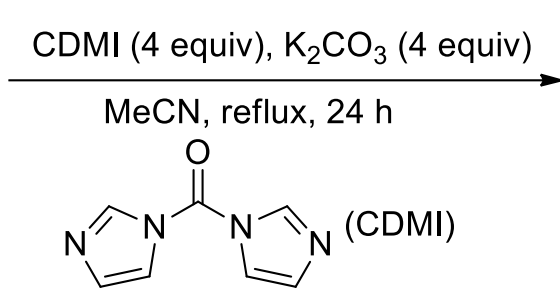<smiles>FC(F)(F)c1nnc([Al])c(C(F)(F)F)c1[18F]</smiles>

To a Teflon screw-capped Schlenk tube with a magnetic stirring bar, $0.1 \mathrm{mmol}$ of $\mathbf{2}$, CDMI (64.8 mg, $0.4 \mathrm{mmol}, 4.0$ equiv), $\mathrm{K}_{2} \mathrm{CO}_{3}$ (55.2 mg, $0.4 \mathrm{mmol}, 4.0$ equiv) and 2 $\mathrm{mL}$ dry $\mathrm{MeCN}$ was added. The tube was heated to $120{ }^{\circ} \mathrm{C}$ in oil bath for 24 hours. After the reaction was completed (monitored by TLC), the resulting suspension was extracted with DCM $(3 \times 10 \mathrm{~mL})$, then the organic extracts were combined, dried over $\mathrm{MgSO}_{4}$, filtered and concentrated in vacuo. After being concentrated, the residue was purified by flash column chromatography on silica gel (eluting with petroleum ether/ethyl acetate $=50 / 1)$ to give the product 6 .<smiles>FC(F)(F)c1nnc(-c2ccccc2)c(C(F)(F)F)c1-c1ccccc1</smiles>

3,5-Diphenyl-4,6-bis(trifluoromethyl)pyridazine (6a): white solid; $27.7 \mathrm{mg} ; 75 \%$ yield; m.p. $135-136{ }^{\circ} \mathrm{C} ; \mathrm{R}_{\mathrm{f}}=0.47(\mathrm{PE} / \mathrm{EA}=10 / 1)$; ${ }^{1} \mathbf{H}$ NMR (600 MHz, CDCl$) \delta 7.65(\mathrm{~d}, J=6.9 \mathrm{~Hz}, 2 \mathrm{H}), 7.59-7.44$ (m, 6H), 7.32 (d, J=7.4 Hz, 2H). ${ }^{13}$ C NMR (151 MHz, CDCl $) \delta 161.3$ (s), 149.2 (q, $J=32.6 \mathrm{~Hz}$ ), 139.4 (dd, $J=4.4,2.2 \mathrm{~Hz}), 136.2$ (s), 130.3 (s), 130.2 (s), 129.8 (s), $129.2(\mathrm{dd}, J=3.7,1.6 \mathrm{~Hz}), 128.6$ (s), 128.6 (s), 128.5 (s), 128.0 (s), 122.3 (q, $J=$ $278.0 \mathrm{~Hz}), 121.4(\mathrm{q}, J=275.1 \mathrm{~Hz}) .{ }^{19} \mathbf{F}$ NMR (565 MHz, CDCl 3$) \delta-50.98(\mathrm{~s}, 3 \mathrm{~F})$, $-60.21\left(\mathrm{~s}, 3 \mathrm{~F}\right.$ ). HRMS (ESI-TOF) $\mathrm{m} / z:[\mathrm{M}+\mathrm{H}]$ calcd for $\mathrm{C}_{18} \mathrm{H}_{11} \mathrm{~N}_{2} \mathrm{~F}_{6}$ 369.0826, found 369.0823 .<smiles>Cc1cccc(-c2nnc(C(F)(F)F)c(-c3ccccc3)c2C(F)(F)F)c1</smiles>
5-Phenyl-6-(m-tolyl)-3,5-bis(trifluoromethyl)pyridazine (6b): white solid; $24.1 \mathrm{mg} ; 63 \%$ yield; m.p. $127-128{ }^{\circ} \mathrm{C} ; \mathrm{R}_{\mathrm{f}}$ $=0.46(\mathrm{PE} / \mathrm{EA}=10 / 1) ;{ }^{1} \mathbf{H}$ NMR $\left(\mathbf{6 0 0} \mathbf{~ M H z}, \mathbf{C D C l}_{3}\right) \delta 7.52$ 
$(\mathrm{dd}, J=6.1,3.8 \mathrm{~Hz}, 1 \mathrm{H}), 7.48(\mathrm{dd}, J=9.3,5.2 \mathrm{~Hz}, 3 \mathrm{H}), 7.42(\mathrm{~d}, J=5.0 \mathrm{~Hz}, 2 \mathrm{H}), 7.39$ $-7.36(\mathrm{~m}, 1 \mathrm{H}), 7.32(\mathrm{~d}, J=7.5 \mathrm{~Hz}, 2 \mathrm{H}), 2.46$ (s, 3H). ${ }^{13} \mathbf{C}$ NMR (151 MHz, CDCl$)$ $\delta 161.4(\mathrm{~s}), 149.2$ (q, $J=32.2 \mathrm{~Hz}), 139.4$ (dd, $J=3.6,1.7 \mathrm{~Hz}), 138.4$ (s), 136.2 (s), 131.0 (s), 130.4 (s), 129.8 (s), 129.7 (s), 129.6 (s), 128.6 (d, J = 1.3 Hz), 128.4 (s), $128.0(\mathrm{~s}), 126.3(\mathrm{dd}, J=4.3,2.1 \mathrm{~Hz}), 122.3(\mathrm{q}, J=278.3 \mathrm{~Hz}), 121.4(\mathrm{q}, J=276.7 \mathrm{~Hz})$, 21.5 (s). ${ }^{19}$ F NMR (565 MHz, CDCl 3 ) $\delta-50.94$ (s, 3F), -60.24 (s, 3F). HRMS (ESI-TOF) $\boldsymbol{m} / \boldsymbol{z}:[\mathrm{M}+\mathrm{H}]$ calcd for $\mathrm{C}_{19} \mathrm{H}_{13} \mathrm{~N}_{2} \mathrm{~F}_{6}$ 383.0983, found 383.0980.

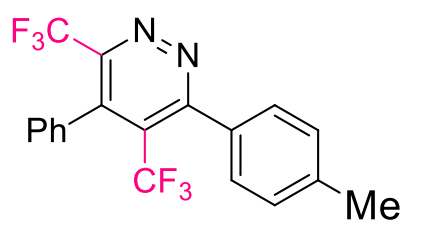

\section{4-Phenyl-6-(p-tolyl)-3,5-bis(trifluoromethyl)pyridazine}

(6c): white solid; $24.1 \mathrm{mg}$; $63 \%$ yield; m.p. $135-136{ }^{\circ} \mathrm{C} ; \mathrm{R}_{\mathrm{f}}$ $7.65(\mathrm{~d}, J=6.9 \mathrm{~Hz}, 2 \mathrm{H}), 7.58-7.49(\mathrm{~m}, 3 \mathrm{H}), 7.29(\mathrm{~d}, J=7.9 \mathrm{~Hz}, 2 \mathrm{H}), 7.20$ (d, $J=$ $7.8 \mathrm{~Hz}, 2 \mathrm{H}), 2.44(\mathrm{~s}, 3 \mathrm{H}) .{ }^{13} \mathbf{C}$ NMR (151 MHz, $\left.\mathbf{C D C l}_{3}\right) \delta 161.2(\mathrm{~s}), 149.4$ (q, J = $32.0 \mathrm{~Hz}$ ), 140.0 (s), 139.8 (dd, $J=4.0,2.2 \mathrm{~Hz}), 136.3$ (s), 130.2 (s), 130.0 (s), 129.2 (q, $J=1.7 \mathrm{~Hz}), 128.7(\mathrm{~s}), 128.5(\mathrm{~s}), 128.5$ (s), 127.3 (s), 122.3 (q, $J=278.1 \mathrm{~Hz})$,

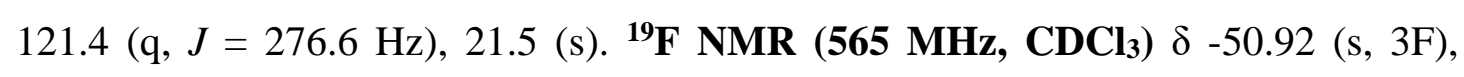
$-60.16\left(\mathrm{~s}, 3 \mathrm{~F}\right.$ ). HRMS (ESI-TOF) $\mathrm{m} / z:[\mathrm{M}+\mathrm{H}]$ calcd for $\mathrm{C}_{19} \mathrm{H}_{13} \mathrm{~N}_{2} \mathrm{~F}_{6}$ 383.0983, found 383.0984 .<smiles>Cc1cccc(-c2c(C(F)(F)F)nnc(-c3ccccc3)c2C(F)(F)F)c1</smiles>

\section{2-Phenyl-5-(m-tolyl)-4,6-bis(trifluoromethyl)pyridazine}

(6d): white solid; $26.4 \mathrm{mg}$; 69\% yield; m.p. $163-164{ }^{\circ} \mathrm{C} ; \mathrm{R}_{\mathrm{f}}=$ $0.46(\mathrm{PE} / \mathrm{EA}=10 / 1) ;{ }^{1} \mathbf{H}$ NMR (600 MHz, $\left.\mathbf{C D C l}_{3}\right) \delta 7.65(\mathrm{~d}$, $J=7.6 \mathrm{~Hz}, 2 \mathrm{H}), 7.59-7.50(\mathrm{~m}, 3 \mathrm{H}), 7.39-7.35(\mathrm{~m}, 1 \mathrm{H}), 7.32(\mathrm{~d}, J=7.2 \mathrm{~Hz}, 1 \mathrm{H})$, 7.12 (s, 2H), 2.42 (s, 3H). ${ }^{13} \mathbf{C}$ NMR (151 MHz, CDCl $) \delta 161.2$ (s), 149.3 (q, $J=$ $32.2 \mathrm{~Hz}$ ), 139.7 (d, $J=1.9 \mathrm{~Hz}$ ), 137.8 (s), 136.3 (s), 130.6 (s), 130.3 (s), 130.2 (s), $129.2(\mathrm{~d}, J=1.4 \mathrm{~Hz}), 128.5$ (s), 127.8 (s), 127.7 (s), 127.6 (s), 125.8 (s), 122.3 (q, $J=$ $278.2 \mathrm{~Hz}), 121.4(\mathrm{q}, J=276.6 \mathrm{~Hz}), 21.4(\mathrm{~s}) .{ }^{19}$ F NMR (565 MHz, CDCl 3 ) $\delta$-50.89 (s, 3F), -60.14 (s, 3F). HRMS (ESI-TOF) $\boldsymbol{m} / z:[\mathrm{M}+\mathrm{H}]$ calcd for $\mathrm{C}_{19} \mathrm{H}_{13} \mathrm{~N}_{2} \mathrm{~F}_{6} 383.0983$, found 383.0984 . 
<smiles>Fc1ccc(-c2c(C(F)(F)F)nnc(-c3ccccc3)c2C(F)(F)F)cc1</smiles>

\section{3-(4-Fluorophenyl)-6-phenyl-3,5-bis(trifluoromethyl)pyrid}

azine (6e): white solid; $25.0 \mathrm{mg}$; $65 \%$ yield; m.p. $119-120^{\circ} \mathrm{C}$;

$\mathrm{R}_{\mathrm{f}}=0.44(\mathrm{PE} / \mathrm{EA}=10 / 1) ;{ }^{1} \mathbf{H}$ NMR $\left(\mathbf{6 0 0} \mathbf{M H z}, \mathbf{C D C l}_{3}\right) \delta$

$7.64(\mathrm{~d}, J=6.4 \mathrm{~Hz}, 2 \mathrm{H}), 7.56(\mathrm{t}, J=8.7 \mathrm{~Hz}, 3 \mathrm{H}), 7.31(\mathrm{~s}, 2 \mathrm{H}), 7.20(\mathrm{t}, J=7.3 \mathrm{~Hz}, 2 \mathrm{H})$. ${ }^{13}$ C NMR (151 MHz, CDCl $) 164.4(\mathrm{~s}), 162.0$ (d, $\left.J=213.5 \mathrm{~Hz}\right), 149.3$ (q, $J=32.4$ Hz), 138.6 (dd, $J=4.8,2.9 \mathrm{~Hz}), 136.0$ (s), 130.7 (d, $J=8.6 \mathrm{~Hz}), 130.4$ (s), 129.2 (dd, $J=3.6,1.9 \mathrm{~Hz}), 128.6(\mathrm{~s}), 126.2(\mathrm{~d}, J=3.9 \mathrm{~Hz}), 122.2(\mathrm{q}, J=278.1 \mathrm{~Hz}), 121.4(\mathrm{q}, J=$ $276.6 \mathrm{~Hz}), 115.6$ (s), 115.4 (s). ${ }^{19} \mathbf{F}$ NMR (565 MHz, CDCl $) \delta$-50.91 (s, 3F), -60.16 (s, 3F), -110.35 (s, 1F). HRMS (ESI-TOF) $\mathbf{m} / z:[\mathrm{M}+\mathrm{H}]$ calcd for $\mathrm{C}_{18} \mathrm{H}_{10} \mathrm{~N}_{2} \mathrm{~F}_{7}$ 387.0732, found 387.0731 .<smiles>FC(F)(F)c1nnc(-c2ccccc2)c(-c2ccc(Cl)cc2)c1C(F)(F)F</smiles>
4-(4-Chlorophenyl)-6-phenyl-3,5-bis(trifluoromethyl)pyri dazine (6f): white solid; $26.1 \mathrm{mg} ; 65 \%$ yield; m.p. $145-146{ }^{\circ} \mathrm{C} ; \mathrm{R}_{\mathrm{f}}=0.44(\mathrm{PE} / \mathrm{EA}=10 / 1) ;{ }^{1} \mathrm{H}$ NMR $(600 \mathrm{MHz}$, $\left.\mathbf{C D C l}_{3}\right) \delta 7.64(\mathrm{~d}, J=7.0 \mathrm{~Hz}, 2 \mathrm{H}), 7.60-7.52(\mathrm{~m}, 3 \mathrm{H}), 7.50-7.46(\mathrm{~m}, 2 \mathrm{H}), 7.27(\mathrm{~d}$, $J=8.3 \mathrm{~Hz}, 2 \mathrm{H}) .{ }^{13} \mathbf{C}$ NMR (151 MHz, $\left.\mathbf{C D C l}_{3}\right) \delta 161.3(\mathrm{~s}), 149.1(\mathrm{q}, J=32.5 \mathrm{~Hz})$, 138.3 (dd, $J=4.4,2.2 \mathrm{~Hz}$ ), 136.3 (s), 131.5 (s), 130.4 (s), 130.0 (s), 129.3 (s), 129.2 (dd, $J=3.9,1.8 \mathrm{~Hz}), 128.7(\mathrm{~s}), 128.6(\mathrm{~s}), 128.5(\mathrm{~s}), 122.2$ (q, $J=278.1 \mathrm{~Hz}), 121.3$ (q, $J=276.5 \mathrm{~Hz}) .{ }^{19} \mathbf{F}$ NMR $(565$ MHz, CDCl 3$) \delta-50.85$ (s, 3F), -60.10 (s, 3F). HRMS (ESI-TOF) $m / z$ : [M+H] calcd for $\mathrm{C}_{18} \mathrm{H}_{10} \mathrm{~N}_{2} \mathrm{~F}_{6} \mathrm{Cl} 403.0437$, found 403.0438 .<smiles>FC(F)(F)c1nnc(-c2ccccc2)c(-c2ccc(Br)cc2)c1C(F)(F)F</smiles>

\section{5-(4-Bromophenyl)-6-phenyl-3,5-bis(trifluoromethyl)pyri} dazine (6g): white solid; $26.8 \mathrm{mg}$; $60 \%$ yield; m.p. $146-147{ }^{\circ} \mathrm{C} ; \mathrm{R}_{\mathrm{f}}=0.44(\mathrm{PE} / \mathrm{EA}=10 / 1) ;{ }^{1} \mathrm{H}$ NMR (600 MHz, $\left.\mathbf{C D C l}_{3}\right) \delta 7.64(\mathrm{~d}, J=6.7 \mathrm{~Hz}, 4 \mathrm{H}), 7.59-7.51(\mathrm{~m}, 3 \mathrm{H}), 7.20(\mathrm{~d}, J=8.2 \mathrm{~Hz}, 2 \mathrm{H}) .{ }^{13} \mathbf{C}$ NMR (151 MHz, CDCl $) \delta 161.3(\mathrm{~s}), 149.0(\mathrm{q}, J=32.5 \mathrm{~Hz}), 138.3(\mathrm{dd}, J=4.3,2.1$ Hz), 136.0 (s), 131.4 (s), 130.4 (s), 130.3 (s), 130.2 (s), 129.2 (s), 129.1 (dd, $J=4.0$, $1.9 \mathrm{~Hz}), 128.6(\mathrm{~s}), 124.5$ (s), $122.2(\mathrm{q}, J=278.3 \mathrm{~Hz}), 121.3(\mathrm{q}, J=276.5 \mathrm{~Hz}) .{ }^{19} \mathbf{F}$ 
NMR (565 MHz, CDCl 3 ) $\delta-50.83$ (s, 3F), -60.07 (s, 3F). HRMS (ESI-TOF) $\boldsymbol{m} / z$ : $[\mathrm{M}+\mathrm{H}]$ calcd for $\mathrm{C}_{18} \mathrm{H}_{10} \mathrm{~N}_{2} \mathrm{~F}_{6} \mathrm{Br} 446.9932$, found 446.9933 .

\section{Possible mechanism of 3,5-ditrifluoromethyl pyridazines 6}

Based on previous studies in the literatures, we proposed a possible mechanism as shown in the following scheme. The $\mathrm{C} 2-\mathrm{C} 3$ bond strength of pyrazoline $\mathbf{2}$ is relatively weak as demonstrated by its bond length (for example, for compound 2a, bond

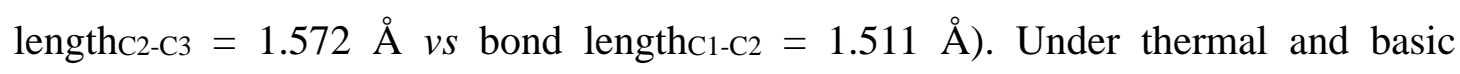
conditions, pyrazoline 2 tends to undergo ring-opening process to generate the hydrazine intermediate Int-A. Subsequently, intramolecular nucleophilic addition closed the ring to form intermediate Int-B. Finally, carbonyldiimidazole (CDMI) might facilitate the elimination of amino moiety under basic conditions, thus producing the observed pyridazine product 6 . However, it should be pointed out that the exact mechanism for this reaction is still not clear and further efforts are in progress in our laboratory.

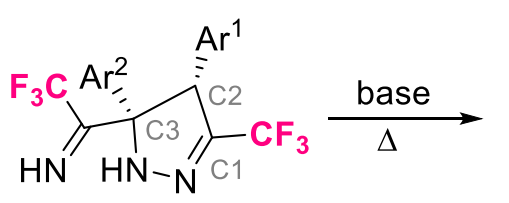

2

Bond length $\mathrm{C} 1-\mathrm{C} 2=1.511 \AA$

Bond length $\mathrm{C} 2-\mathrm{C} 3=1.572 \AA$

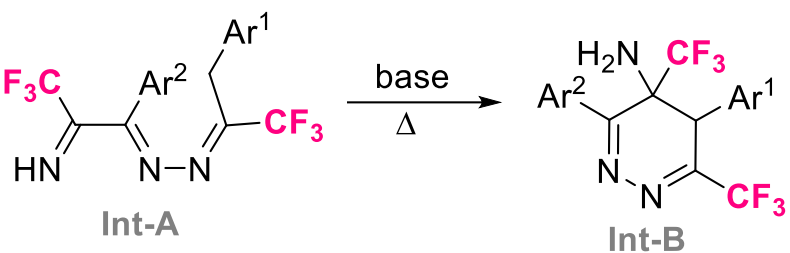

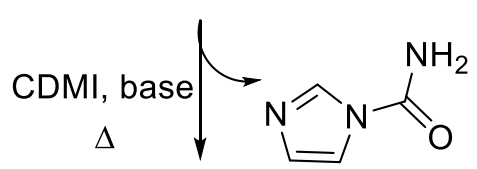<smiles>FC(F)(F)c1nnc(Br)c(C(F)(F)F)c1Br</smiles>

6 


\section{General procedure for the preparation of $\alpha, \beta$-unsaturated trifluoromethyl ketone}

7

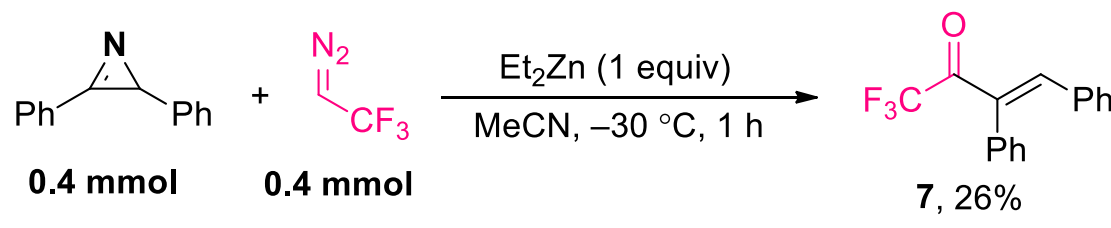

1a

To a $10 \mathrm{~mL}$ Schlenk tube with a magnetic stirring bar, $0.4 \mathrm{mmol}$ of $2 H$-azirine $1 \mathrm{a}$ was added. Then, the tube was degassed and refilled with Argon for 3 times. At $-30{ }^{\circ} \mathrm{C}$, a stock solution of $\mathrm{CF}_{3} \mathrm{CHN}_{2}$ in $\mathrm{MeCN}(0.5 \mathrm{M}, 0.8 \mathrm{~mL}, 1.0$ equiv) was added under Argon. Then $\mathrm{Et}_{2} \mathrm{Zn}(1.0 \mathrm{M}$ in Hexane, $0.4 \mathrm{~mL}, 1.0$ equiv) was added dropwise at $-30{ }^{\circ} \mathrm{C}$ and reacted at $-30{ }^{\circ} \mathrm{C}$ for 1 hour. The reaction was quenched with saturated $\mathrm{NH}_{4} \mathrm{Cl}$. The resulting suspension was extracted with $\mathrm{DCM}(3 \times 10 \mathrm{~mL})$, then the organic extracts were combined, dried over $\mathrm{MgSO}_{4}$, filtered and concentrated in vacuo. After being concentrated, the residue was purified by flash column chromatography on silica gel (eluting with petroleum ether/ethyl acetate $=50 / 1$ ) to give the product 7 .<smiles>O=C(/C(=C\c1ccccc1)C(F)(F)F)c1ccccc1</smiles>

1,1,1-Trifluoro-3,4-diphenylbut-3-en-2-one (7): ${ }^{[3]}$ yellow liquid; $28.6 \mathrm{mg} ; 26 \%$ yield; m.p. $135-136{ }^{\circ} \mathrm{C} ; \mathrm{R}_{\mathrm{f}}=0.53(\mathrm{PE} / \mathrm{EA}=10 / 1) ;{ }^{1} \mathbf{H}$ NMR (600 MHz, CDCl $) \delta 7.84(\mathrm{~s}, 1 \mathrm{H}), 7.46-7.38(\mathrm{~m}, 3 \mathrm{H}), 7.29$ (t, $J=7.4 \mathrm{~Hz}, 1 \mathrm{H}), 7.23-7.15(\mathrm{~m}, 4 \mathrm{H}), 7.06(\mathrm{~d}, J=7.8 \mathrm{~Hz}, 2 \mathrm{H}) .{ }^{13} \mathbf{C}$ NMR $(\mathbf{1 5 1}$ MHz, $\left.\mathbf{C D C l}_{3}\right) \delta 181.5(\mathrm{q}, J=33.6 \mathrm{~Hz}), 146.3(\mathrm{dd}, J=6.3,3.0 \mathrm{~Hz}), 134.9(\mathrm{~s}), 133.8$ (s), 133.6 (s), 131.7 (s), 130.9 (s), 129.9 (s), 129.2 (s), 128.8 (s), 128.6 (s), 117.0 (q, $J$ $=292.2 \mathrm{~Hz}) .{ }^{19} \mathbf{F}$ NMR (565 MHz, CDCl 3$) \delta-69.41(\mathrm{~s}, 3 \mathrm{~F})$. 


\section{References}

1. Zhang, F.-G.; Wei, Y.; Yi, Y.-P.; Nie, J.; Ma, J.-A. Regioselective Cycloaddition of Trifluorodiazoethane with Electron-Deficient Allenic Esters and Ketones: Access to $\mathrm{CF}_{3}$-Substituted Pyrazolines and Pyrazoles. Org. Lett. 2014, 16, 3122.

2. a) Xuan, J.; Xia, X.-D.; Zeng, T.-T.; Feng, Z.-J.; Chen, J.-R.; Lu, L.- Q.; Xiao, W.-J. Visible-light-induced Formal [3+2] Cycloaddition for Pyrrole Synthesis Under Metal-Free Conditions. Angew. Chem., Int. Ed. 2014, 53, 5653-5656. b) Feng F.-F; Li, J.-K.; Liu, X.-Y.; Zhang, F.-G.; Cheung, C.-W; Ma, J.-A. General Synthesis of Tri-Carbo-Substituted $\mathrm{N}_{2}$-Aryl-1,2,3-triazoles via $\mathrm{Cu}$-Catalyzed Annulation of Azirines with Aryldiazonium Salts. J. Org. Chem. 2020, 85, 10872-10883. c) Wang, H.-Y.; Ren, Y.-F.; Wang, K.-Y.; Man, Y.-Q.; Xiang, Y.-An; Li, N.; Tang, B. Visible light-induced cyclization reactions for the synthesis of 1,2,4-triazolines and 1,2,4-triazoles. Chem. Commun., 2017, 53, 9644-9647.

3. Davies, A. T.; Pickett, P. M.; Slawin, A. M. Z.; Smith, A. D. Asymmetric Synthesis of Tri- and Tetrasubstituted Trifluoromethyl Dihydropyranones from a-Aroyloxyaldehydes via NHC Redox Catalysis. ACS Catal. 2014, 4, 2696. 
NMR spectra of the related compounds

${ }^{1} \mathrm{H}$ NMR $\left(600 \mathrm{MHz}, \mathrm{CDCl}_{3}\right)$ spectrum of $\mathbf{2 a}$

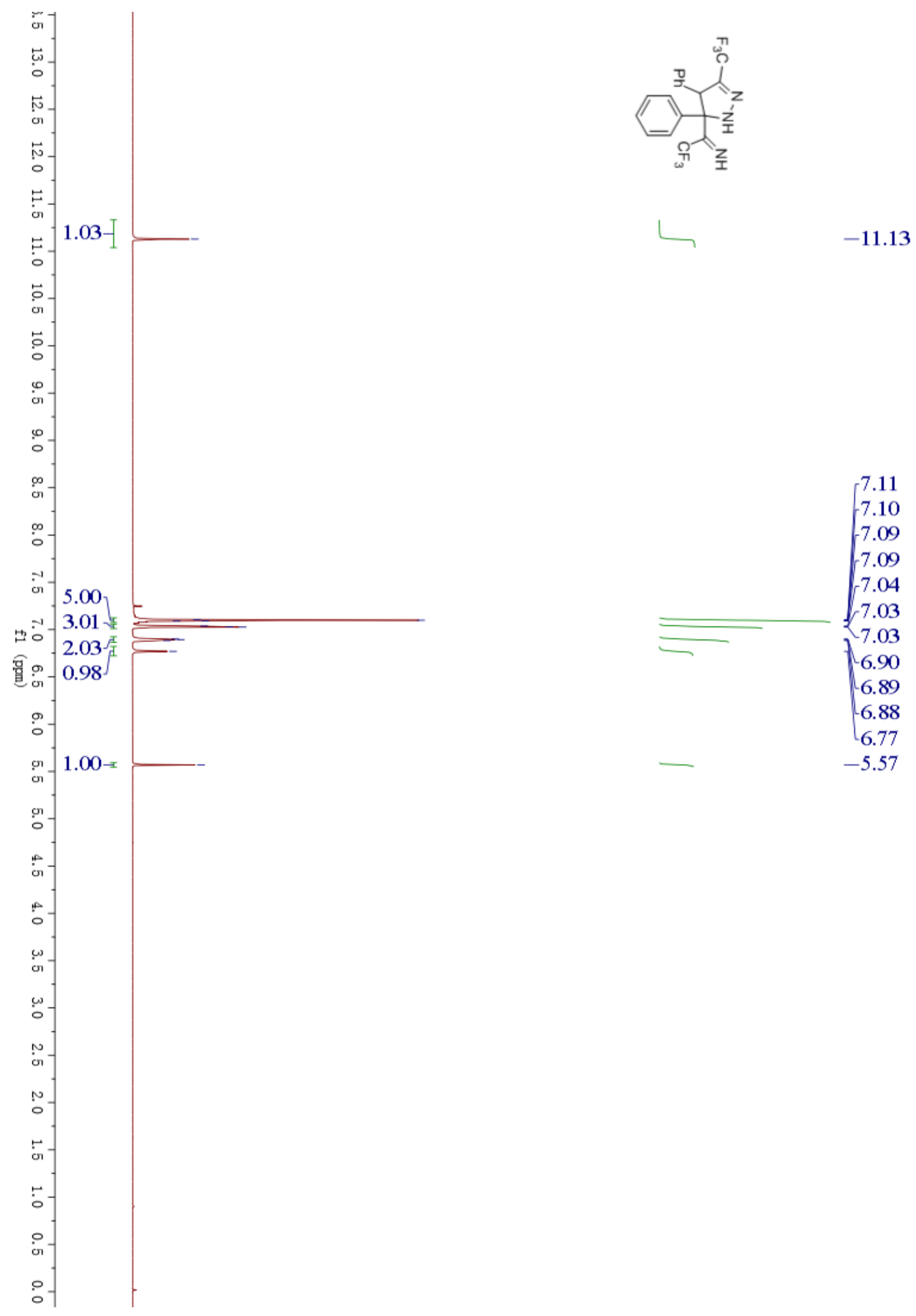


${ }^{13} \mathrm{C} \mathrm{NMR}\left(151 \mathrm{MHz}, \mathrm{CDCl}_{3}\right)$ spectrum of $\mathbf{2 a}$
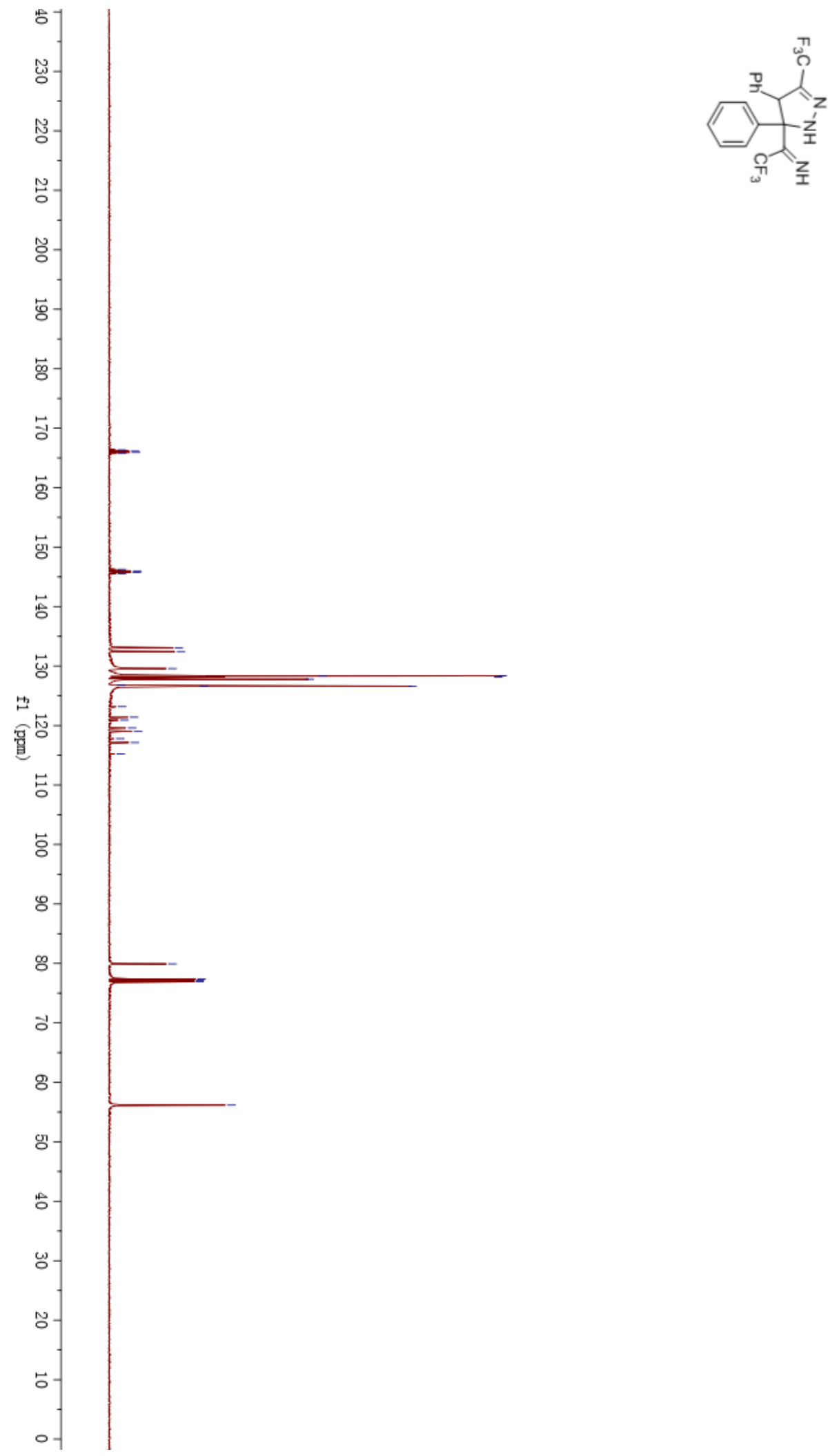
${ }^{19} \mathrm{~F} \mathrm{NMR}\left(565 \mathrm{MHz}, \mathrm{CDCl}_{3}\right)$ spectrum of $\mathbf{2 a}$
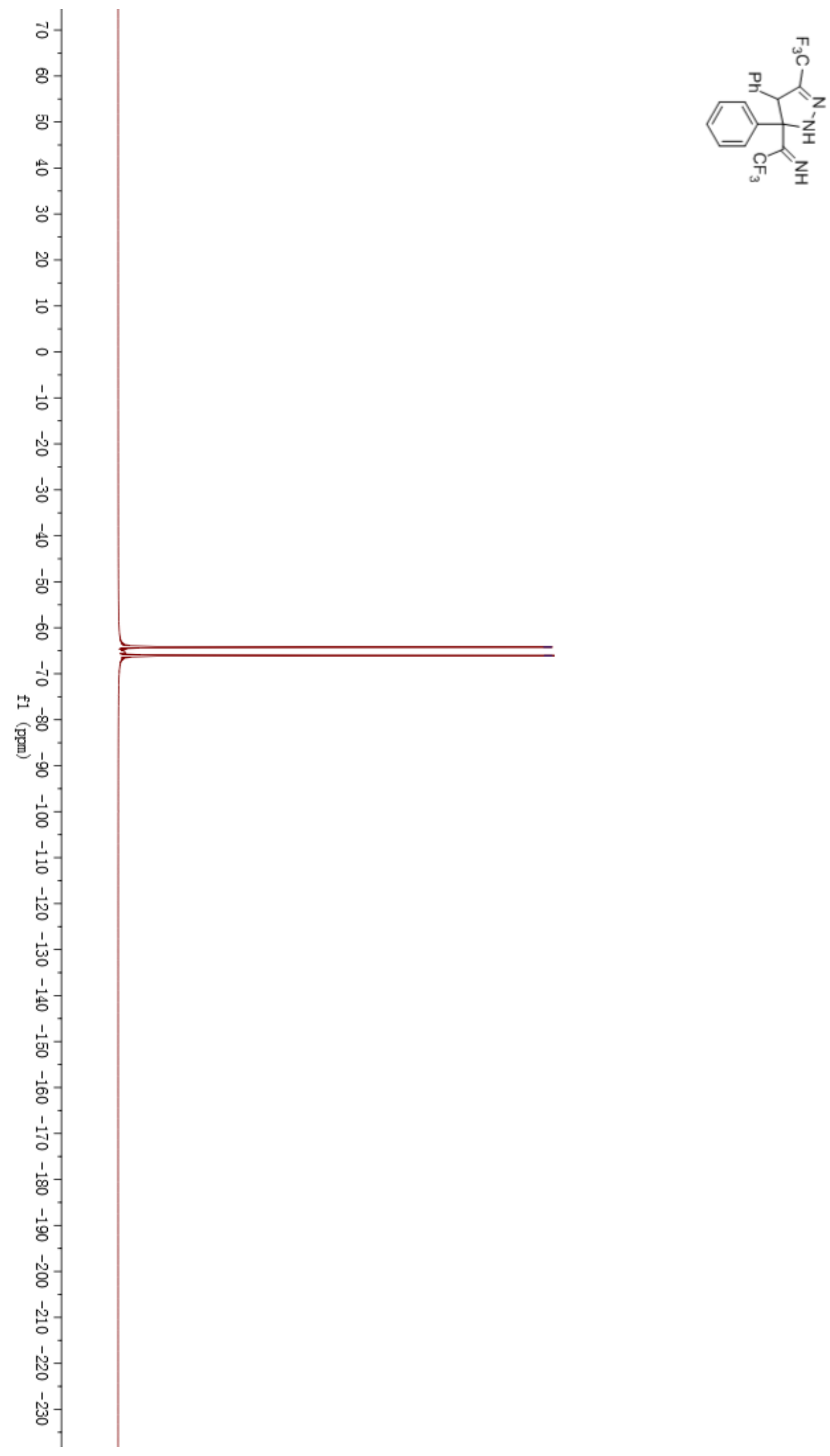
${ }^{1} \mathrm{H}$ NMR (600 MHz, $\mathrm{CDCl}_{3}$ ) spectrum of $\mathbf{2 b}$
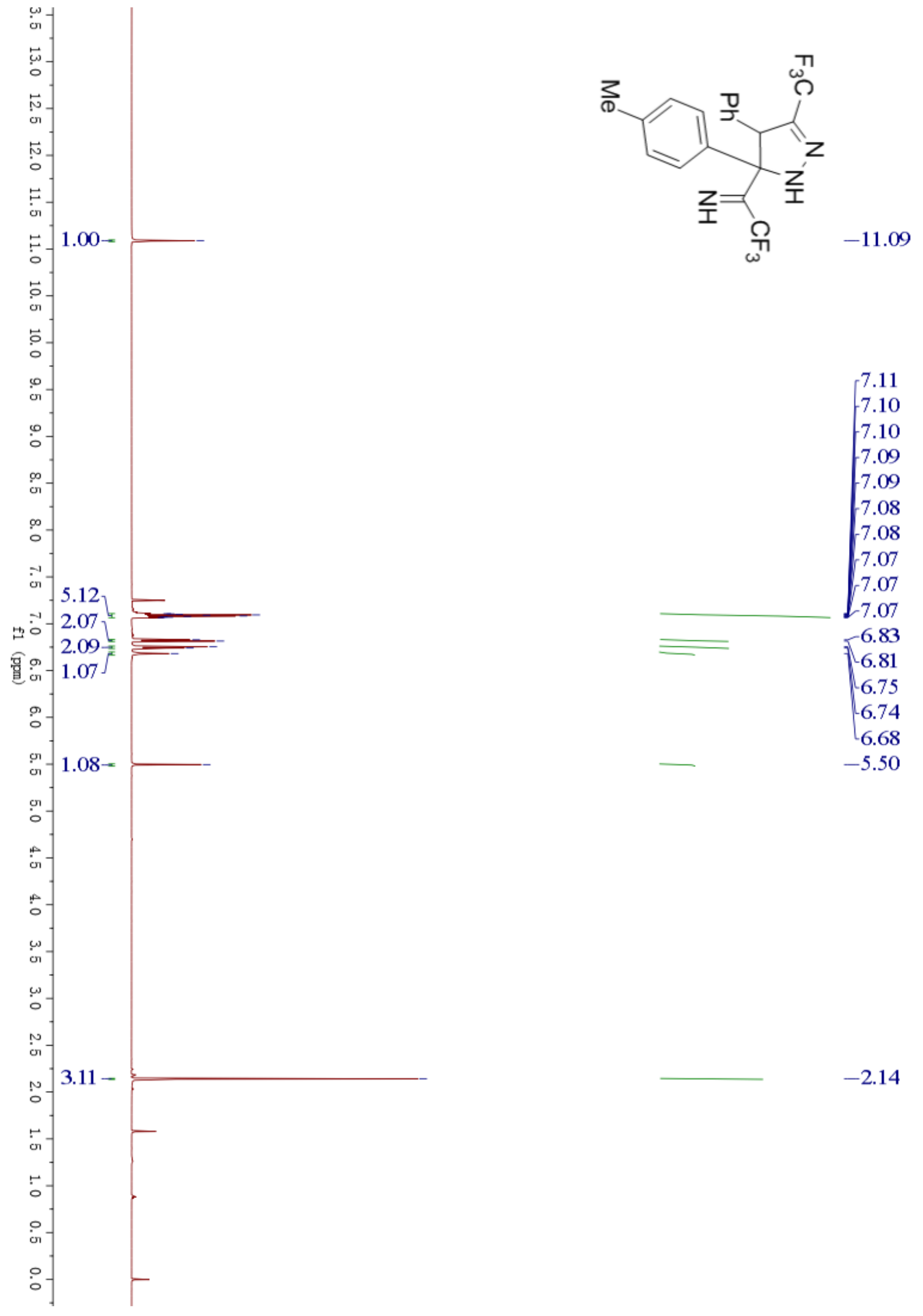

$-2.14$ 
${ }^{13} \mathrm{C} \mathrm{NMR}\left(151 \mathrm{MHz}, \mathrm{CDCl}_{3}\right)$ spectrum of $\mathbf{2 b}$
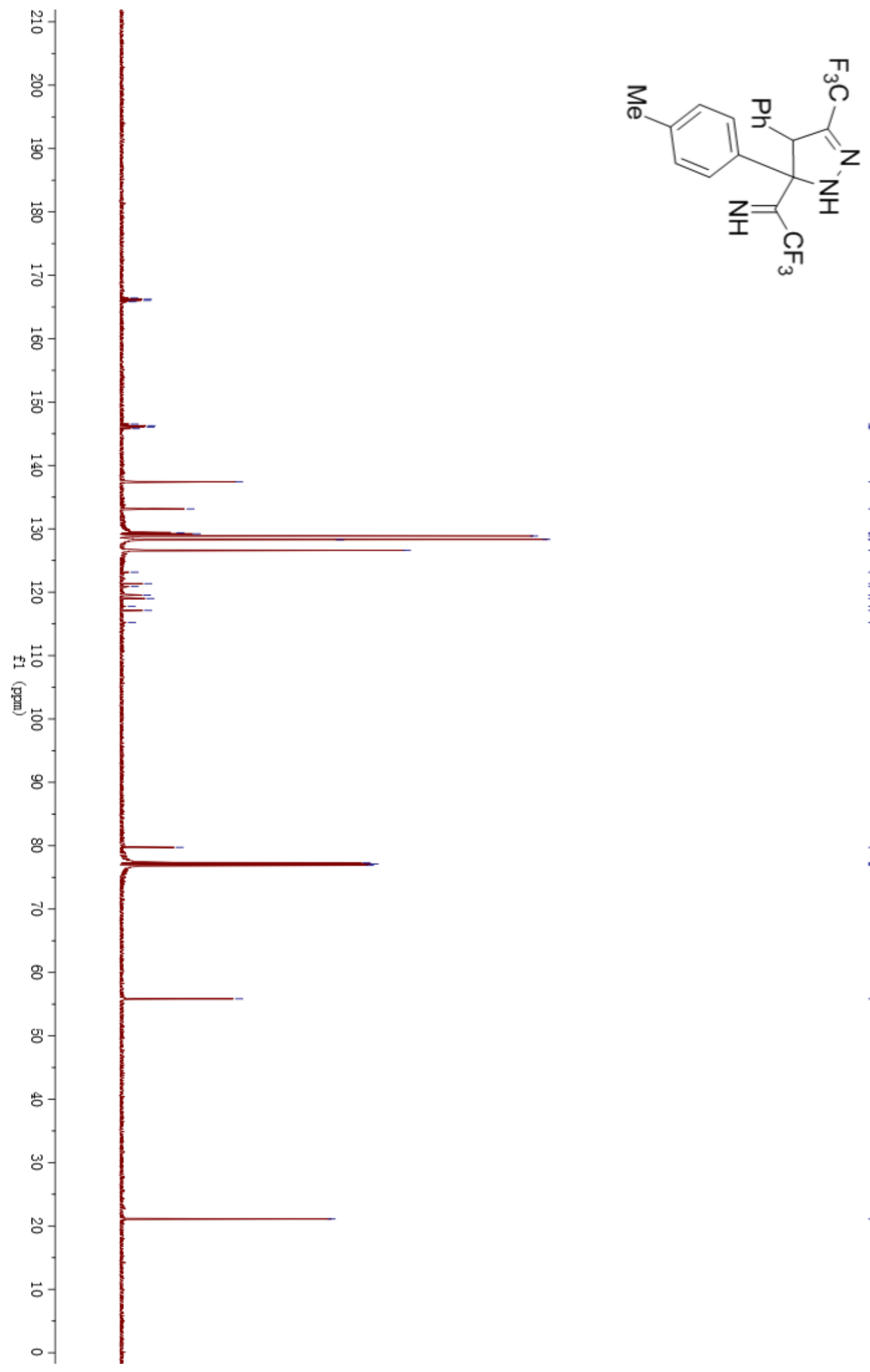

166.41

$-166.22$

166.03

$-165.83$

146.53

146.29

146.05

145.81

137.44

$-133.15$

129.39

129.22

128.87

$-128.33$

128.28

126.62

123.15

121.35

$-120.90$

$-119.56$

119.01

117.76

$-117.12$

115.23

79.74

$-77.33$

77.12

76.90

55.84

$-21.10$ 
${ }^{19} \mathrm{~F}$ NMR (565 MHz, $\left.\mathrm{CDCl}_{3}\right)$ spectrum of $\mathbf{2 b}$

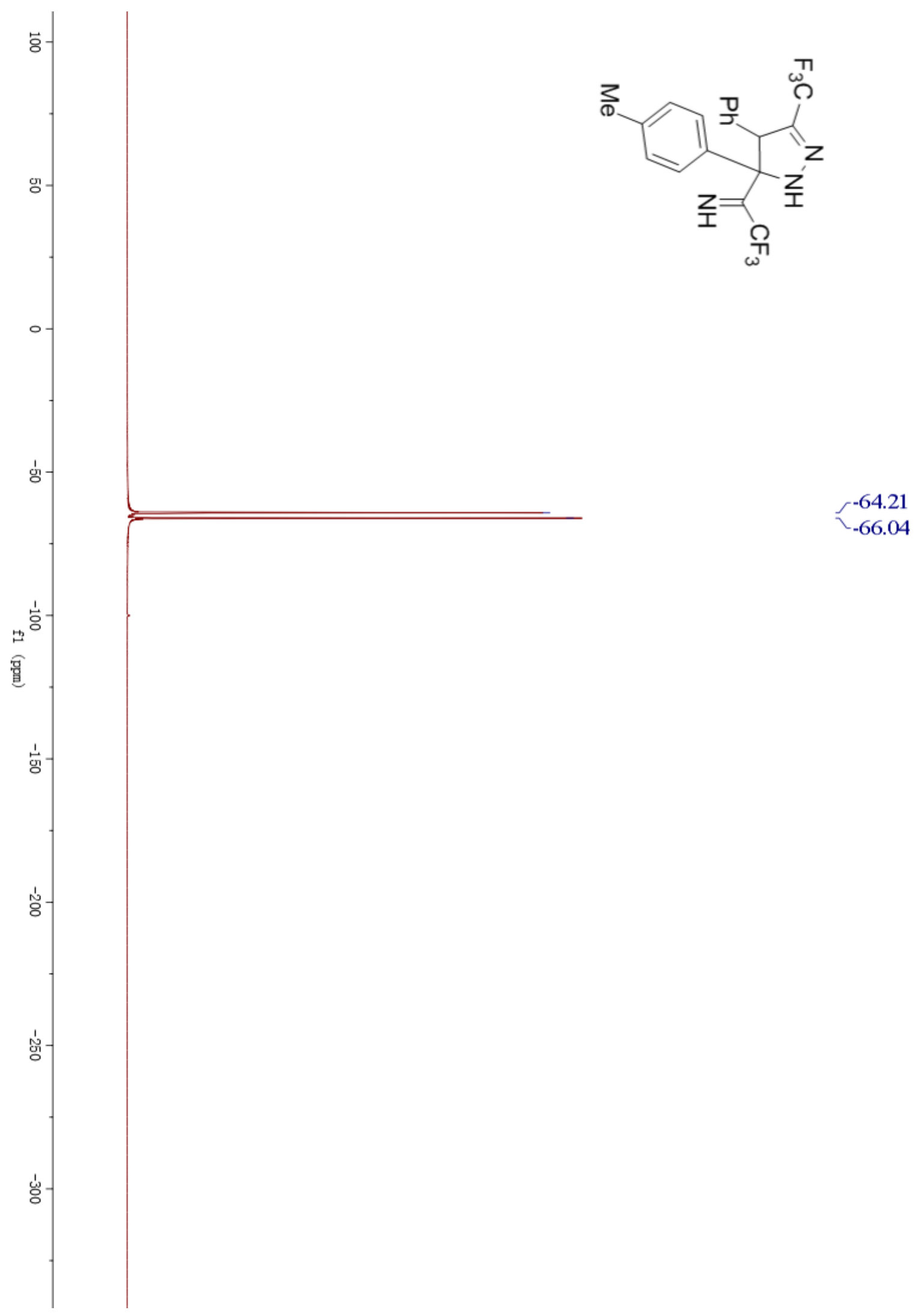


${ }^{1} \mathrm{H} \mathrm{NMR}\left(600 \mathrm{MHz}, \mathrm{CDCl}_{3}\right)$ spectrum of $\mathbf{2 c}$
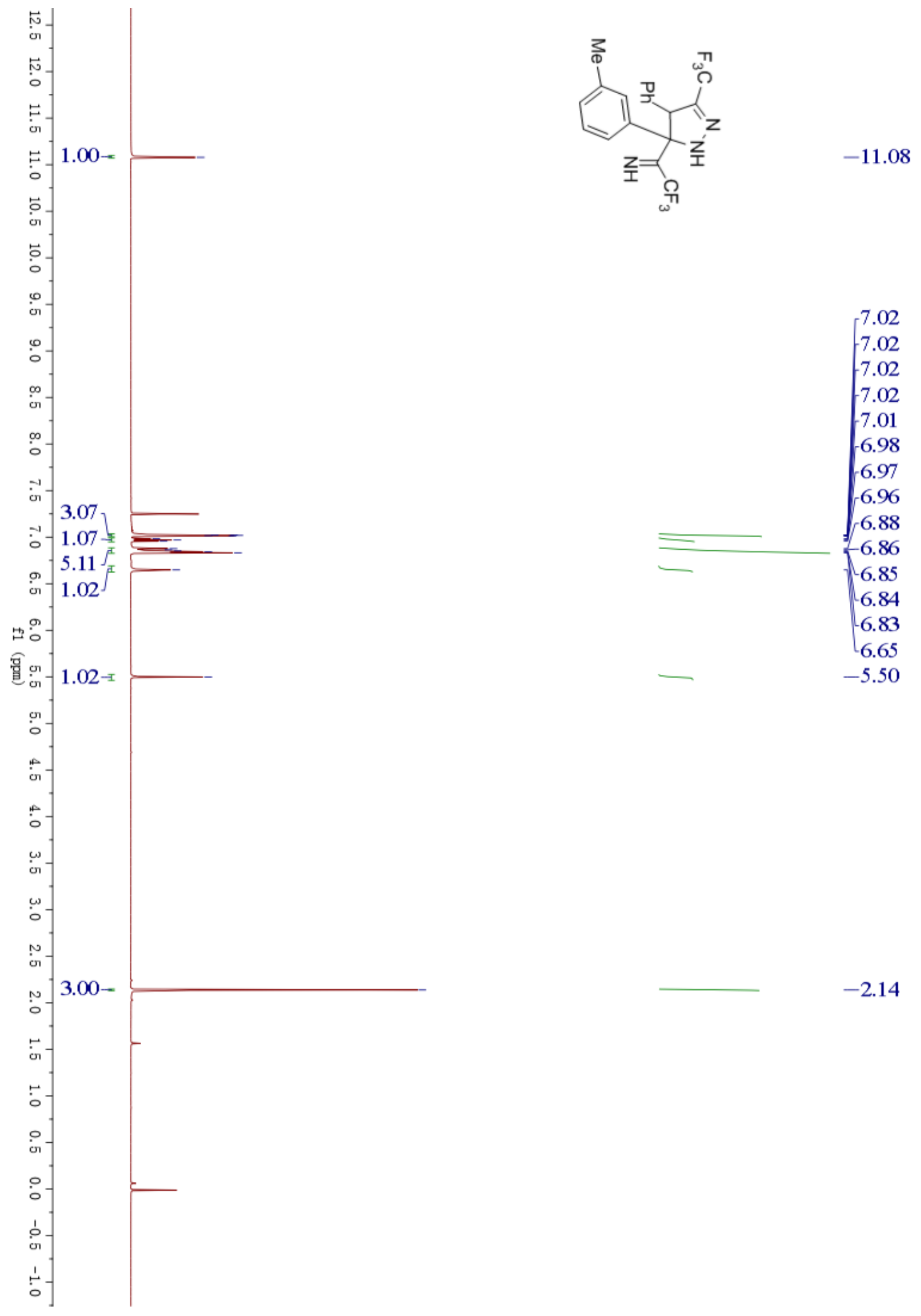

$-2.14$ 
${ }^{13} \mathrm{C} \mathrm{NMR}\left(151 \mathrm{MHz}, \mathrm{CDCl}_{3}\right)$ spectrum of $\mathbf{2 c}$
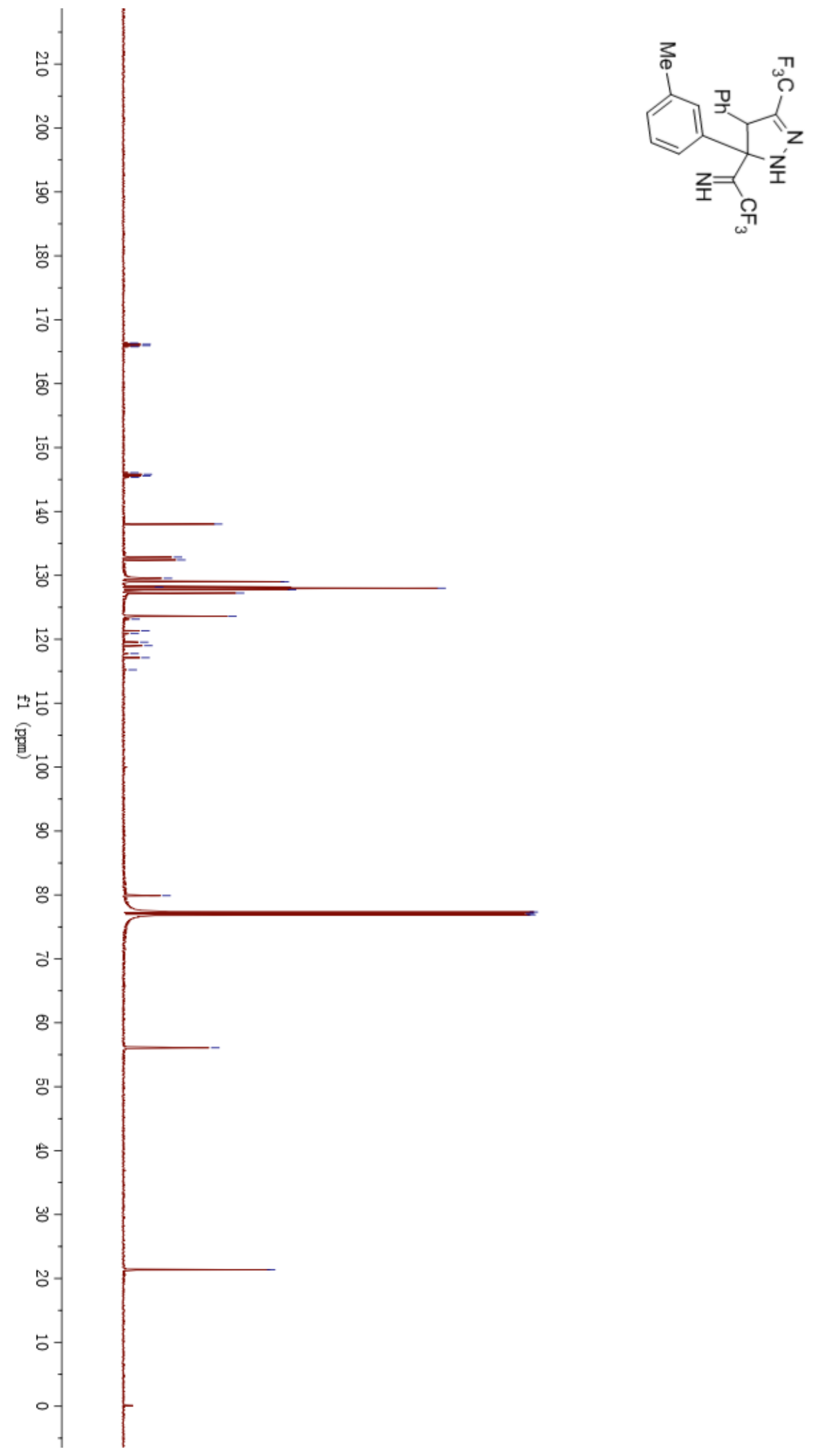

$\left[\begin{array}{l}166.35 \\ 166.16 \\ -165.97\end{array}\right.$

165.77

146.07

$-145.83$

145.58

$-145.34$

138.03

$-132.85$

$-132.42$

$-129.54$

129.02

128.18

128.00

127.74

$-127.24$

123.60

123.14

121.35

120.90

$-119.55$

119.01

$-117.75$

117.12

115.23

79.89

f 77.33

77.12

76.91

$-56.09$

$-21.34$ 
${ }^{19} \mathrm{~F}$ NMR (565 MHz, $\mathrm{CDCl}_{3}$ ) spectrum of $\mathbf{2 c}$

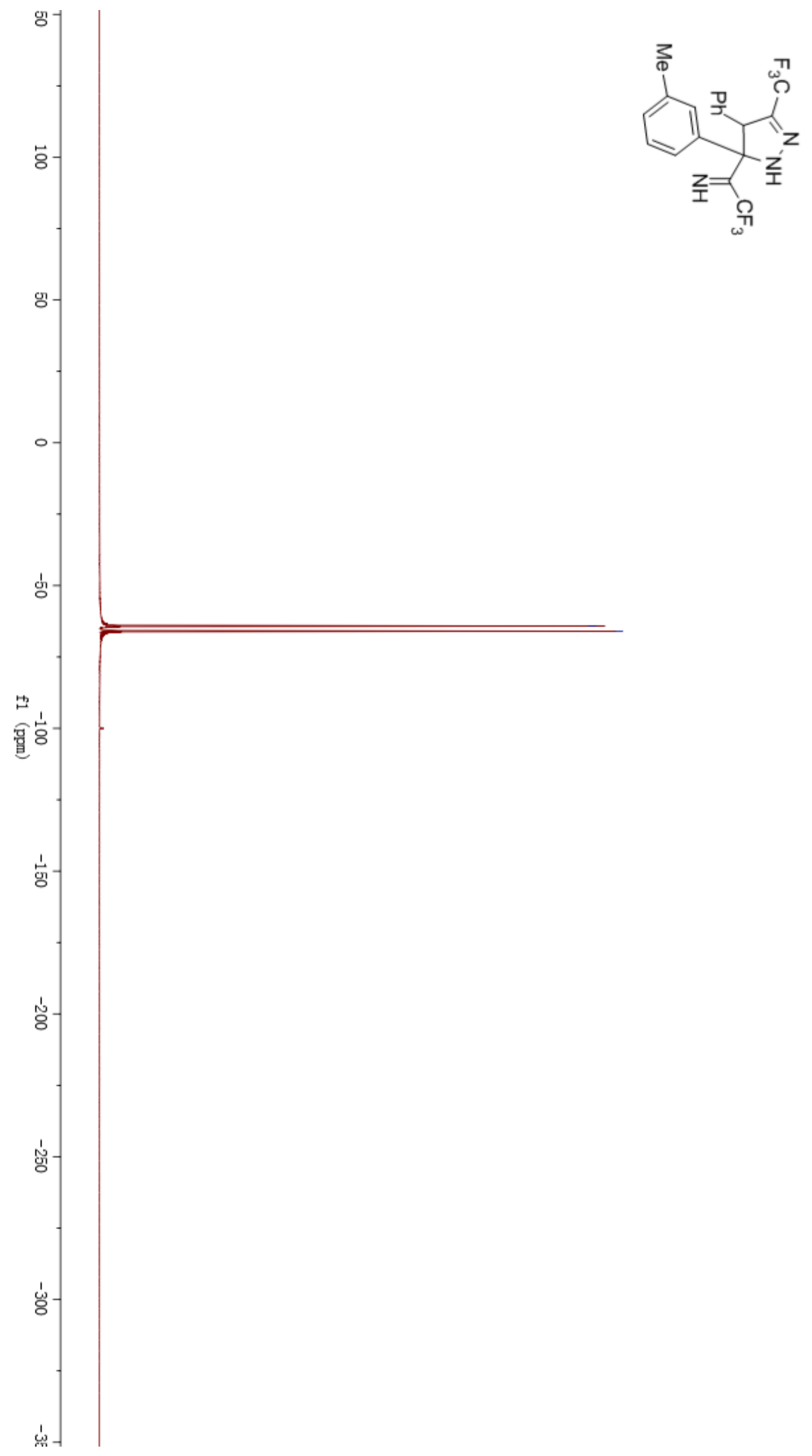


${ }^{1} \mathrm{H}$ NMR $\left(600 \mathrm{MHz}, \mathrm{CDCl}_{3}\right)$ spectrum of $\mathbf{2 d}$
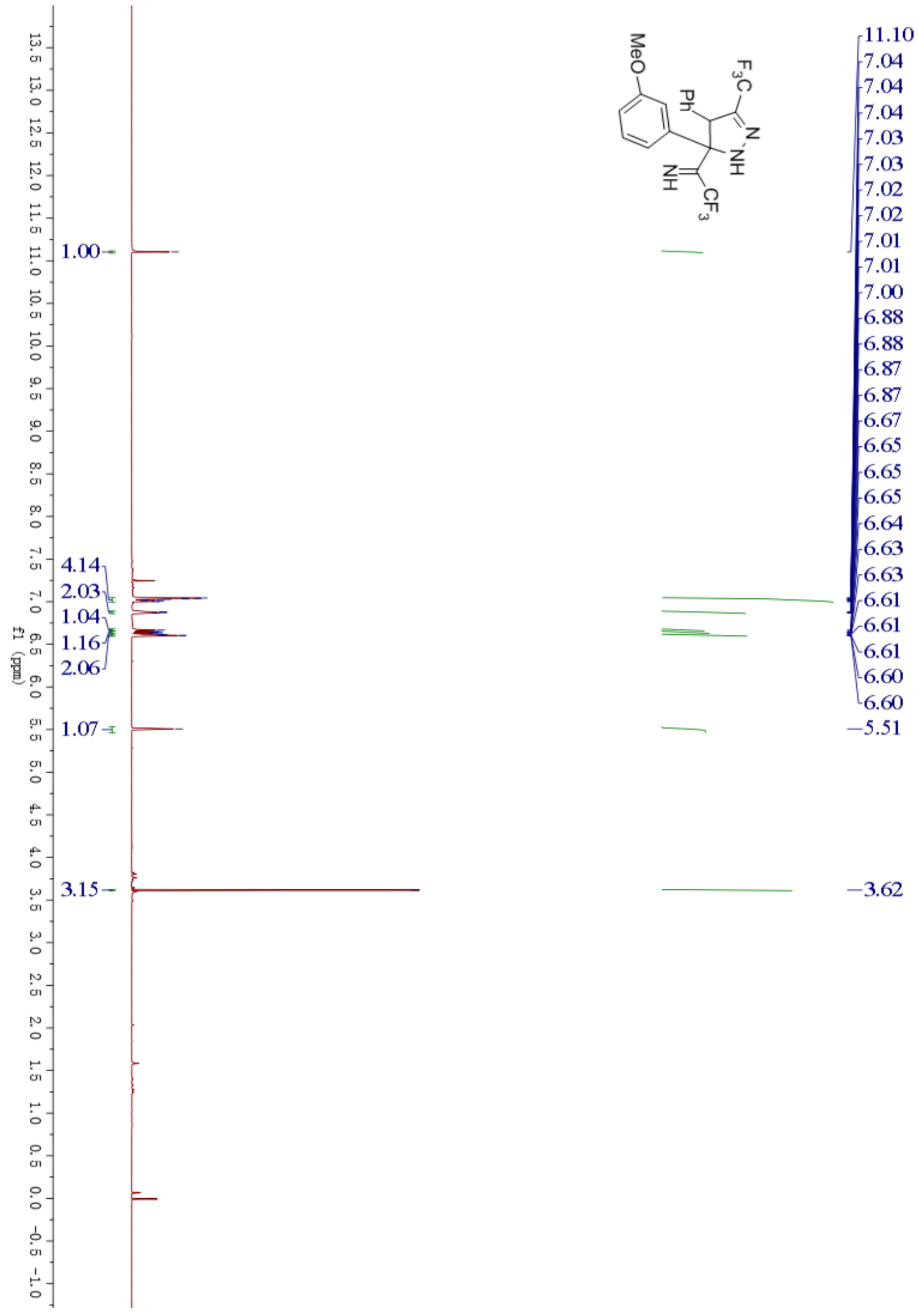
${ }^{13} \mathrm{C} \mathrm{NMR}\left(151 \mathrm{MHz}, \mathrm{CDCl}_{3}\right)$ spectrum of $\mathbf{2 d}$
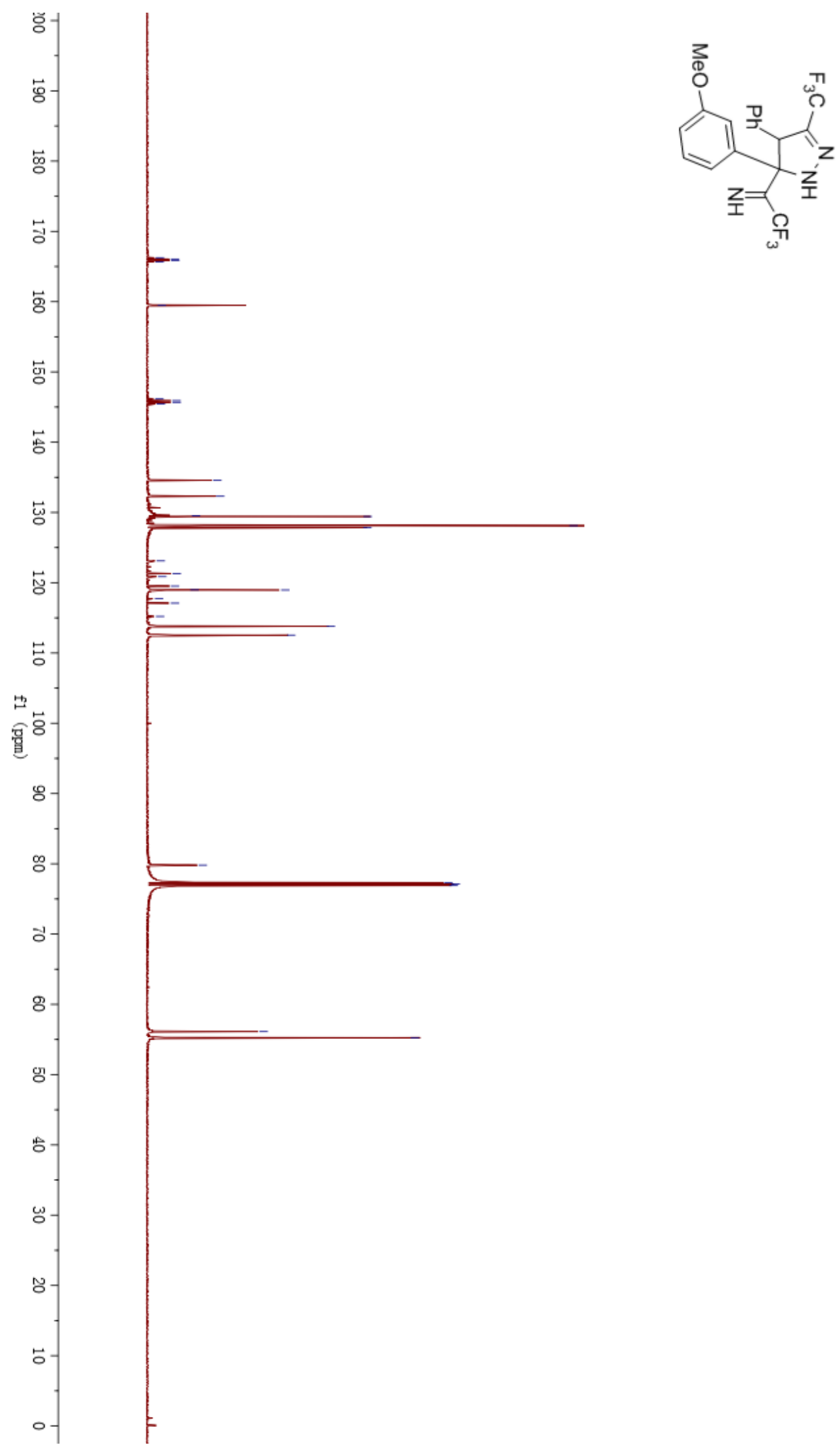

$\left\{\begin{array}{l}166.25 \\ 166.06 \\ -165.87 \\ 165.68 \\ 159.46\end{array}\right.$

146.16

145.91

$-145.67$

145.43

134.59

132.30

$-129.54$

129.42

128.12

127.83

$-123.11$

$-121.31$

$-120.87$

$-119.52$

$-118.99$

118.96

$-117.72$

$-117.09$

$-115.20$

$-113.82$

112.54

79.81

$-77.33$

77.12

76.91

$-56.12$

55.26 
${ }^{19} \mathrm{~F} \mathrm{NMR}\left(565 \mathrm{MHz}, \mathrm{CDCl}_{3}\right)$ spectrum of $\mathbf{2 d}$

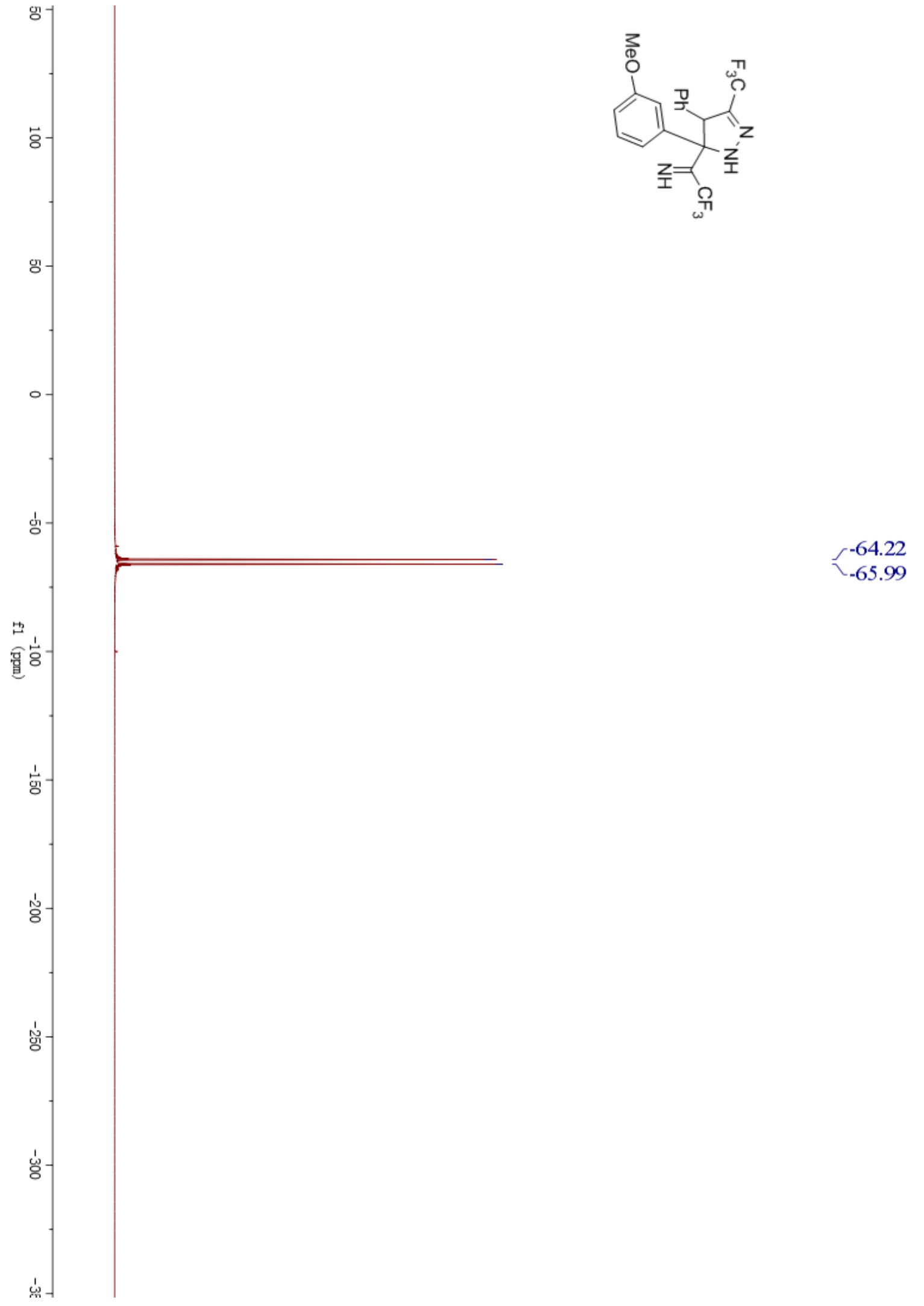


${ }^{1} \mathrm{H} \mathrm{NMR}\left(600 \mathrm{MHz}, \mathrm{CDCl}_{3}\right)$ spectrum of $\mathbf{2 e}$

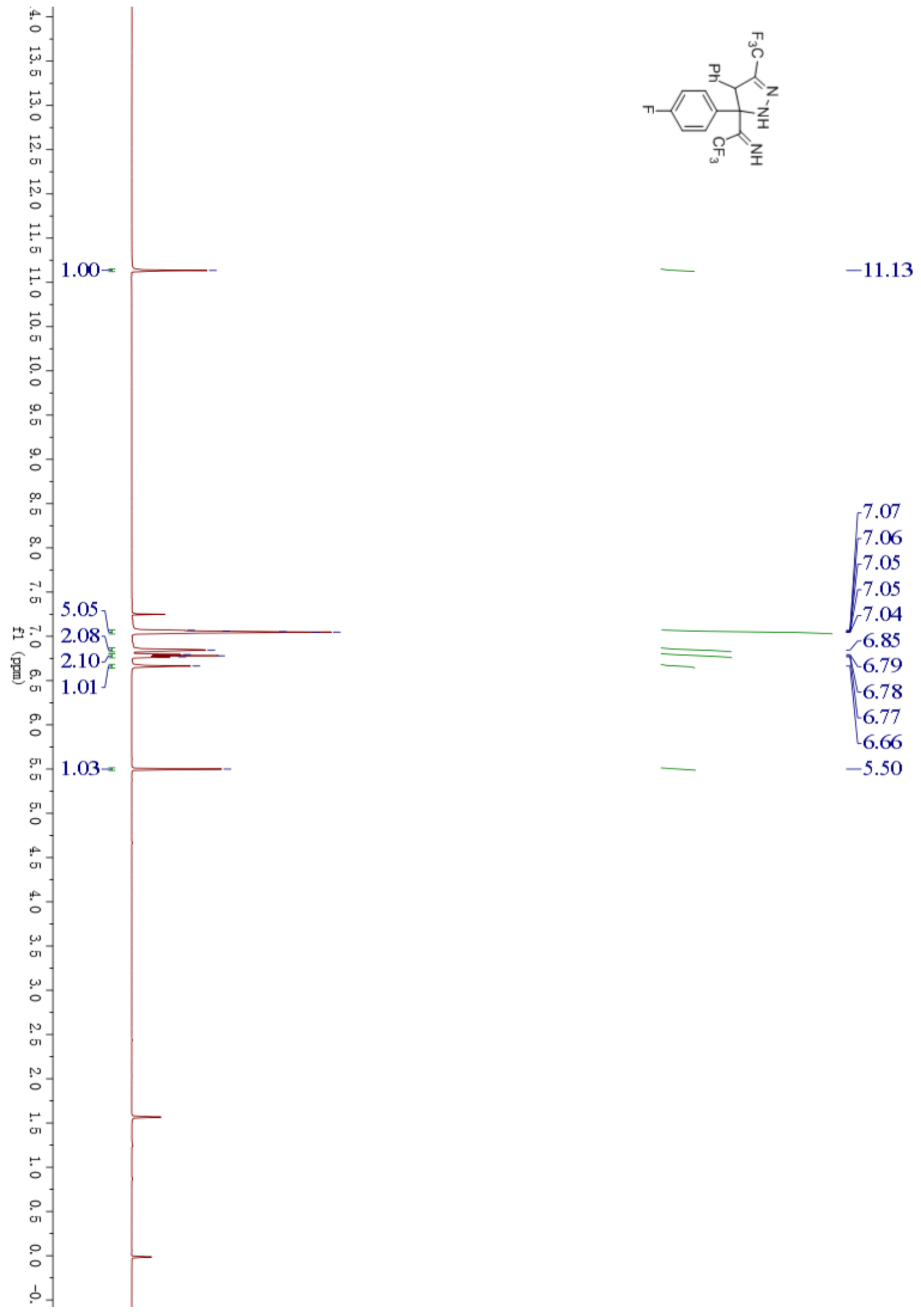


${ }^{13} \mathrm{C}$ NMR (151 MHz, $\mathrm{CDCl}_{3}$ ) spectrum of $\mathbf{2 e}$
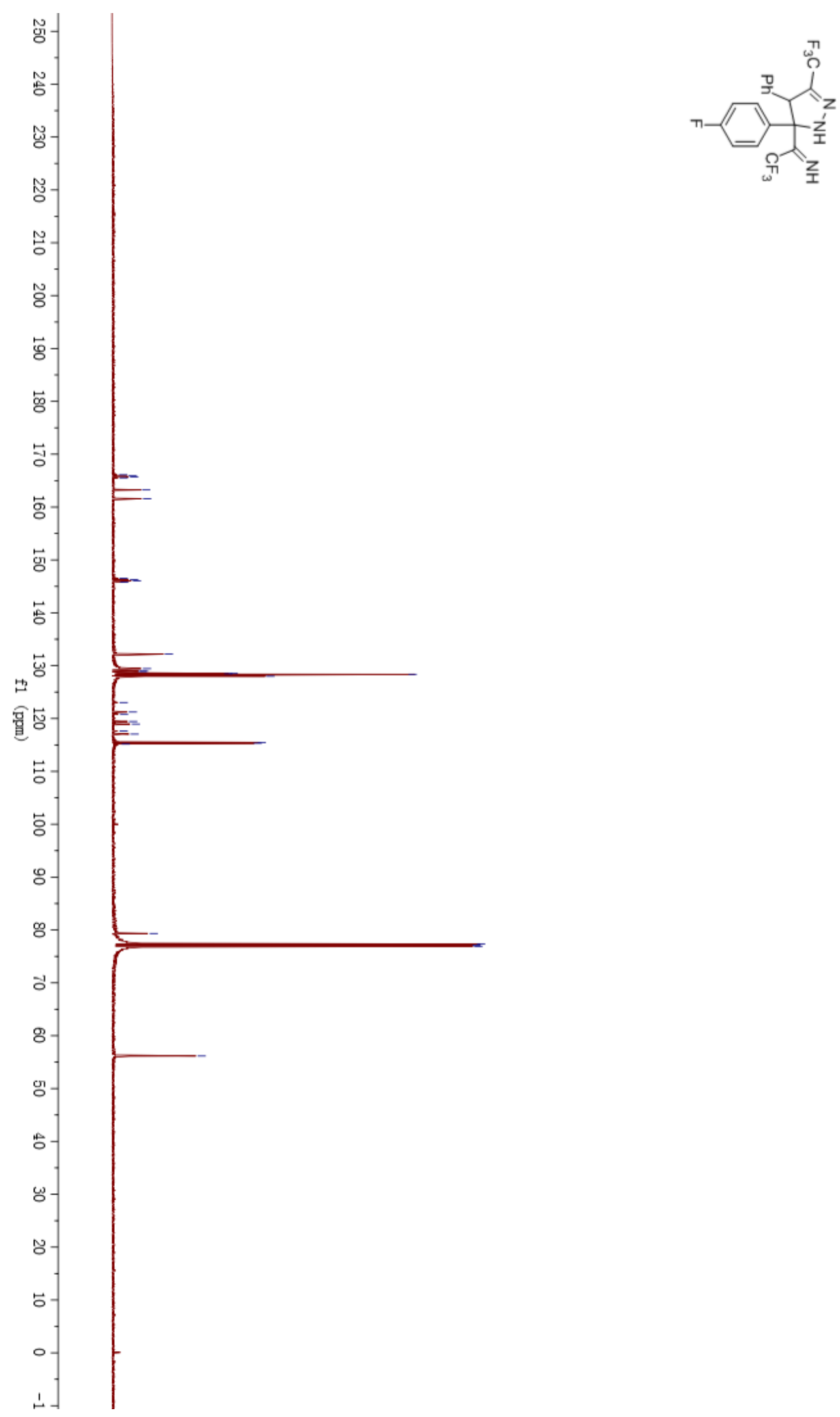

166.10 165.91 165.72 $-165.53$ 163.24

161.60 146.53 $-146.29$ 146.05 145.80 132.19 $-129.43$ $-128.96$ 128.93 128.52 128.46 128.35 128.00 123.02 121.23 120.83 $-119.43$ 118.94 $-117.63$ $-117.05$ $-115.48$ $-115.33$ $-115.16$ $-79.33$

$-77.32$

77.11

$-76.90$

56.16 
${ }^{19} \mathrm{~F}$ NMR $\left(565 \mathrm{MHz}, \mathrm{CDCl}_{3}\right)$ spectrum of $\mathbf{2 e}$

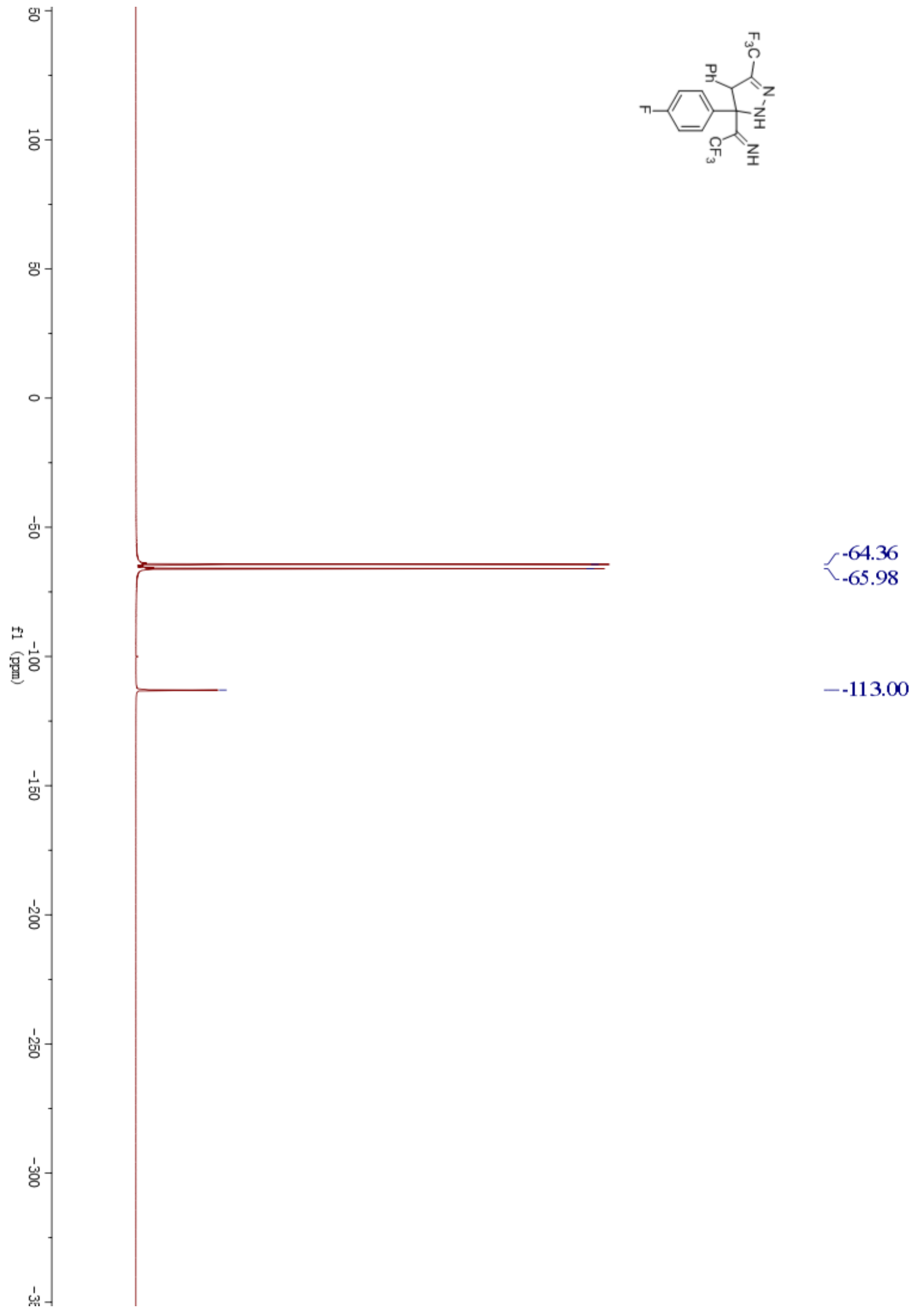


${ }^{1} \mathrm{H}$ NMR (600 MHz, $\left.\mathrm{CDCl}_{3}\right)$ spectrum of $\mathbf{2 f}$

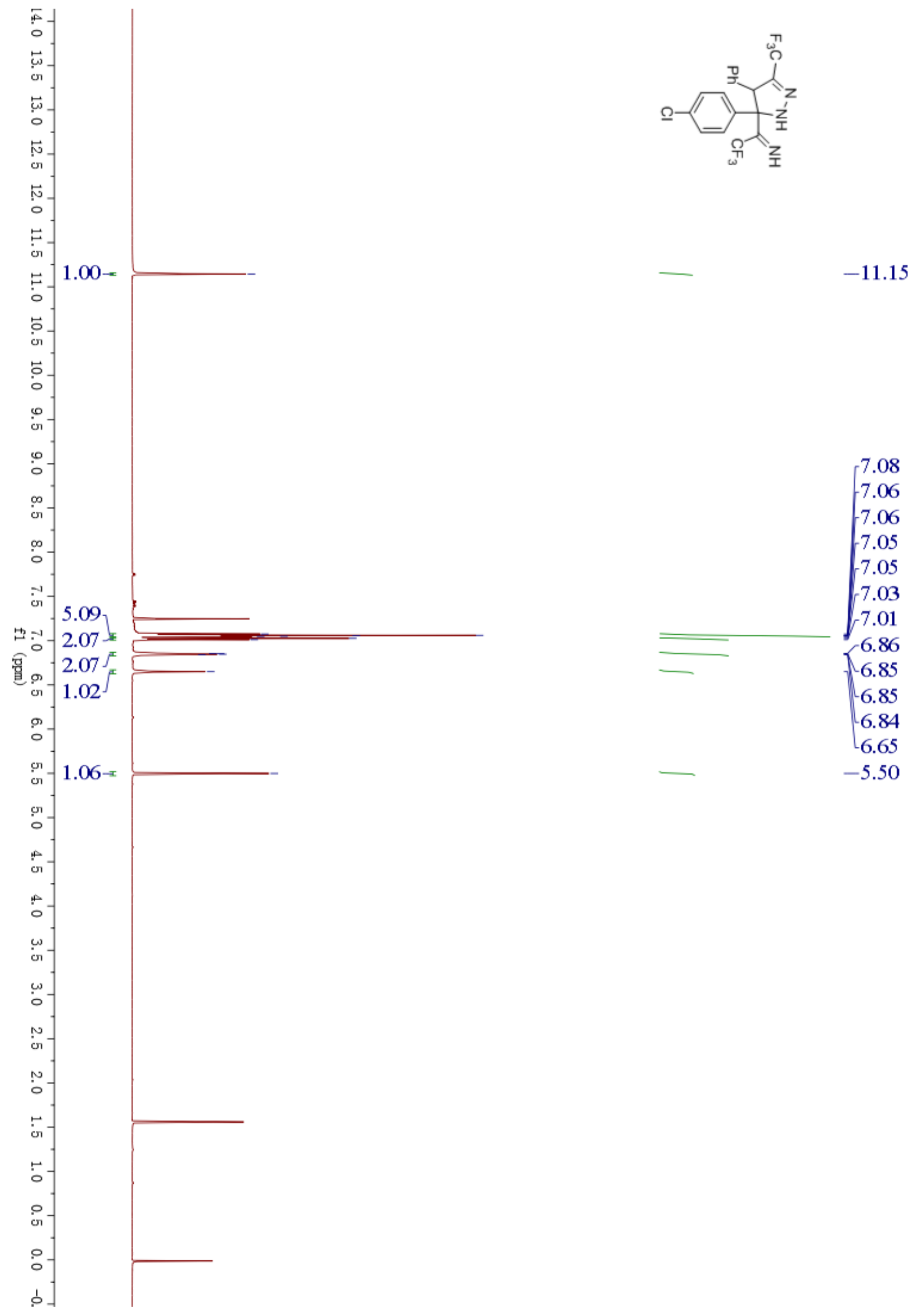


${ }^{13} \mathrm{C} \mathrm{NMR}\left(151 \mathrm{MHz}, \mathrm{CDCl}_{3}\right)$ spectrum of $\mathbf{2 f}$

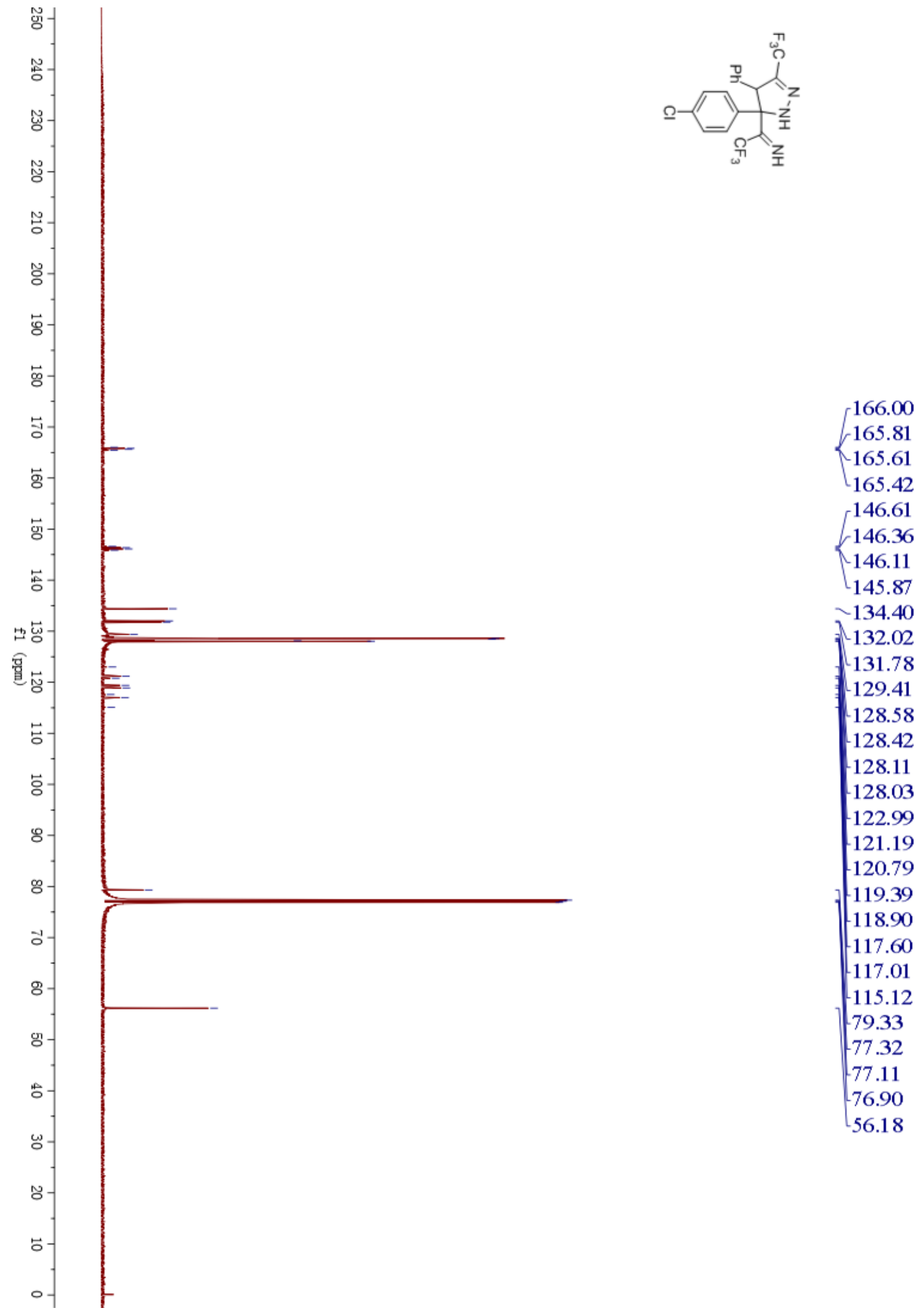


${ }^{19} \mathrm{~F}$ NMR (565 MHz, $\mathrm{CDCl}_{3}$ ) spectrum of $\mathbf{2 f}$

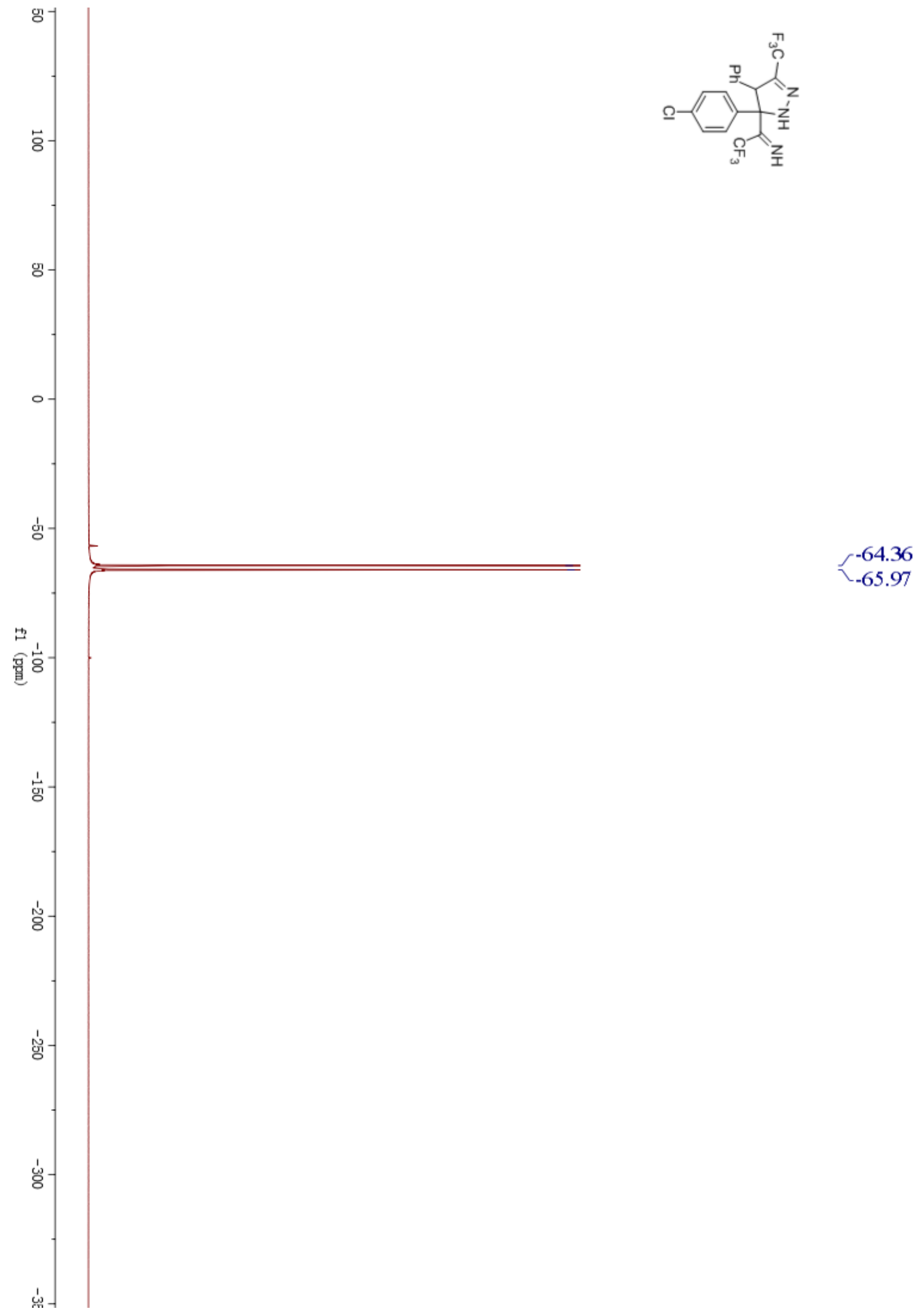


${ }^{1} \mathrm{H}$ NMR (600 MHz, $\mathrm{CDCl}_{3}$ ) spectrum of $\mathbf{2 g}$
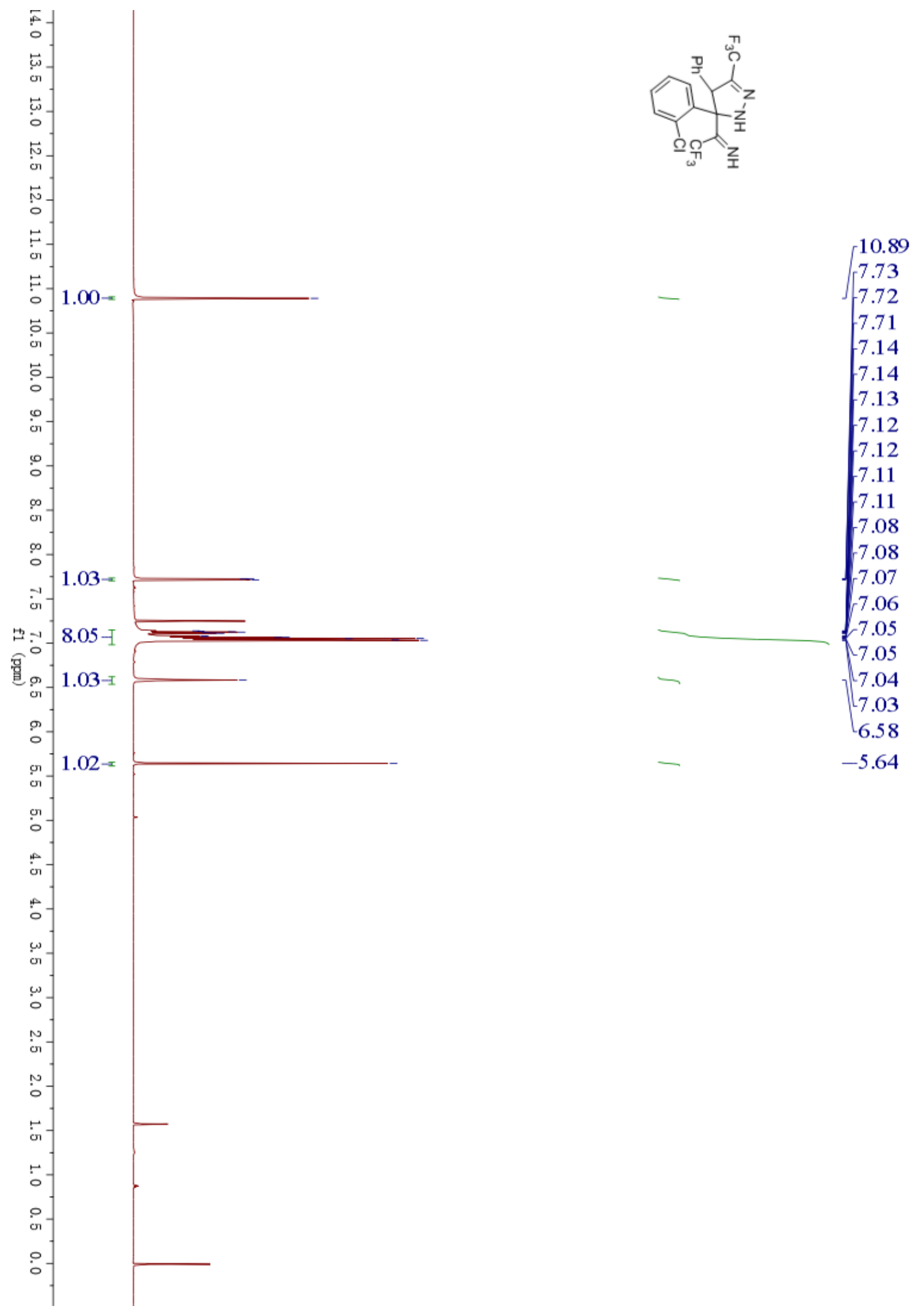
${ }^{13} \mathrm{C}$ NMR (151 MHz, $\mathrm{CDCl}_{3}$ ) spectrum of $\mathbf{2 g}$

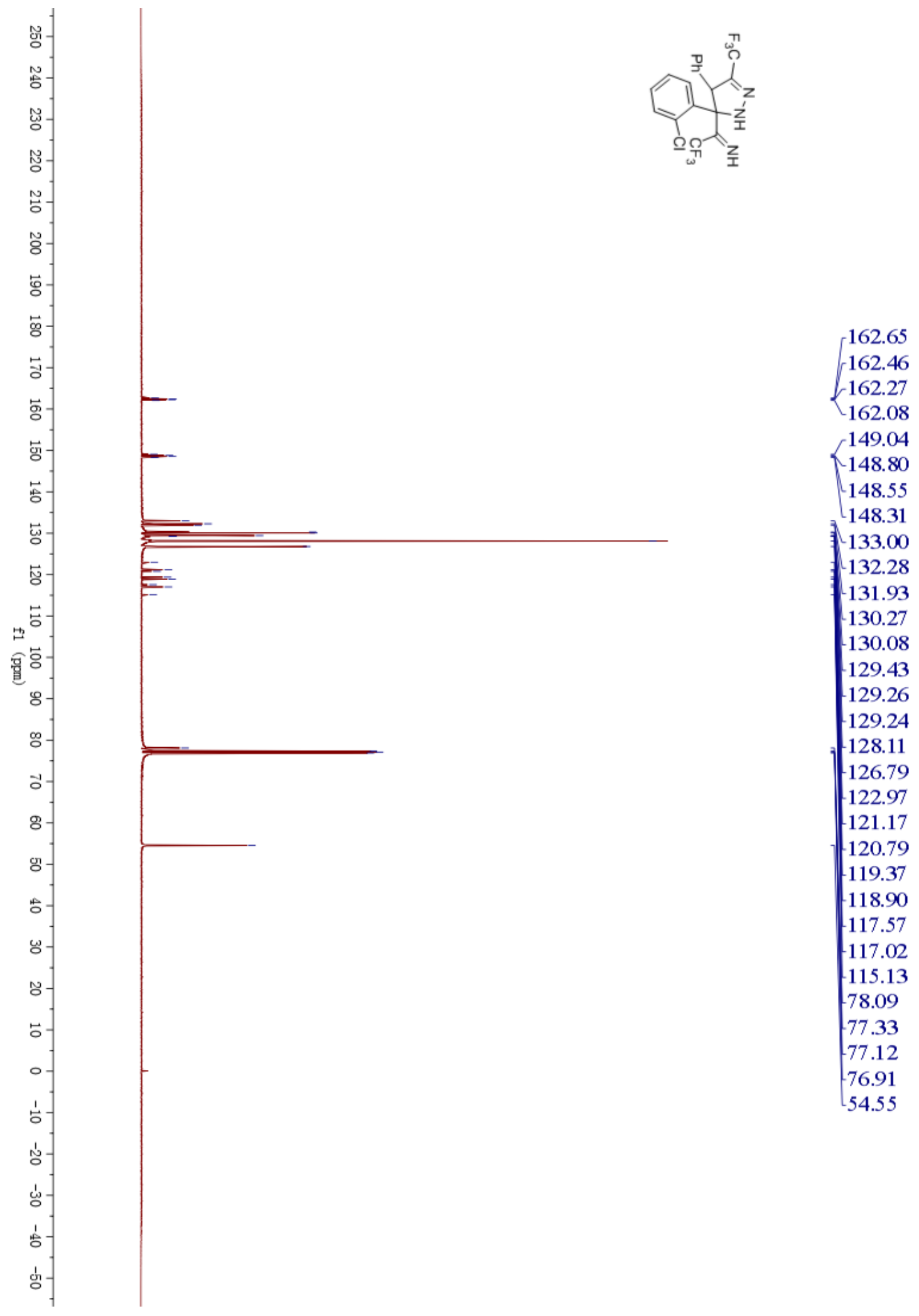


${ }^{19} \mathrm{~F} \mathrm{NMR}\left(565 \mathrm{MHz}, \mathrm{CDCl}_{3}\right)$ spectrum of $\mathbf{2 g}$

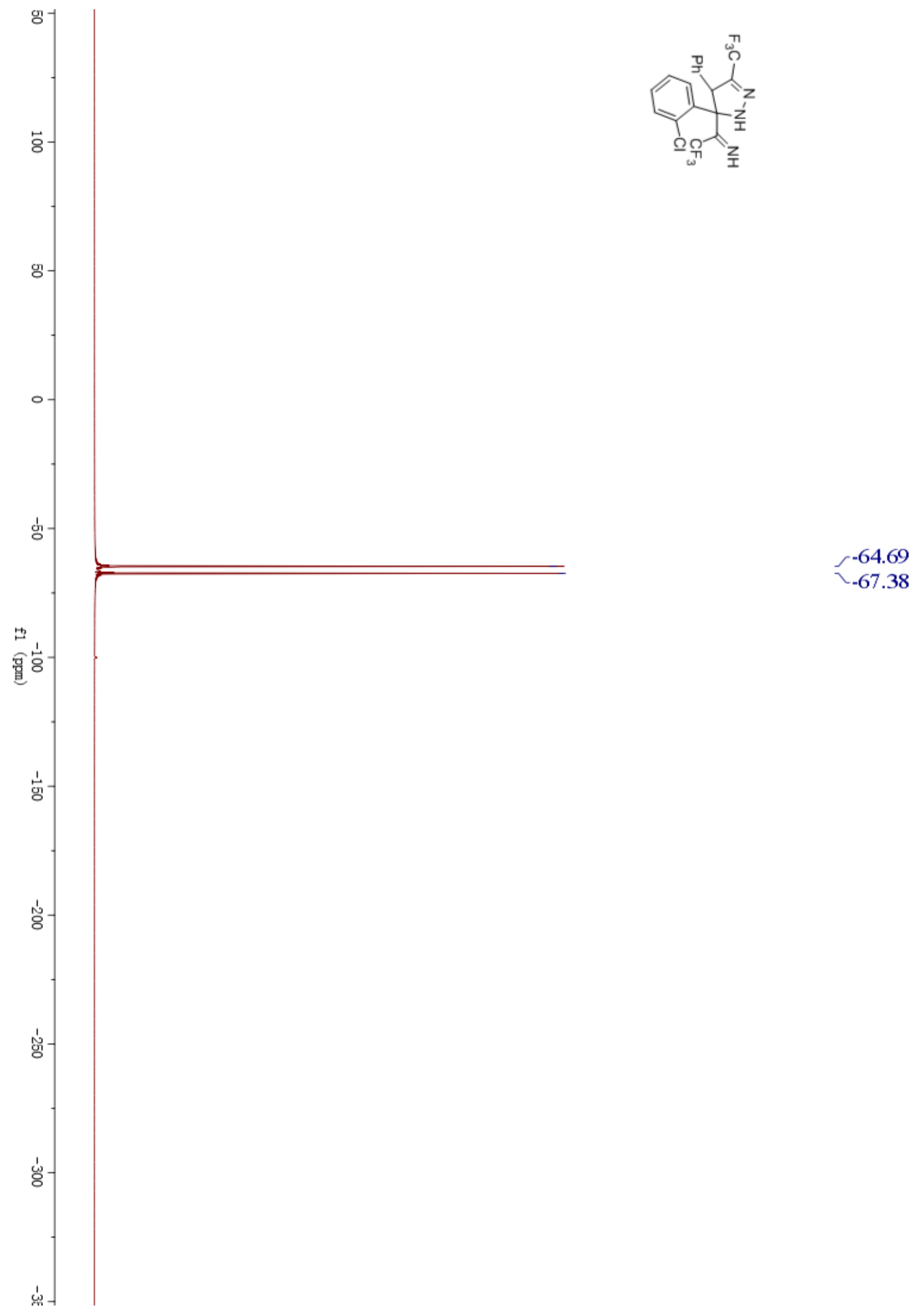


${ }^{1} \mathrm{H}$ NMR $\left(600 \mathrm{MHz}, \mathrm{CDCl}_{3}\right)$ spectrum of $\mathbf{2 h}$
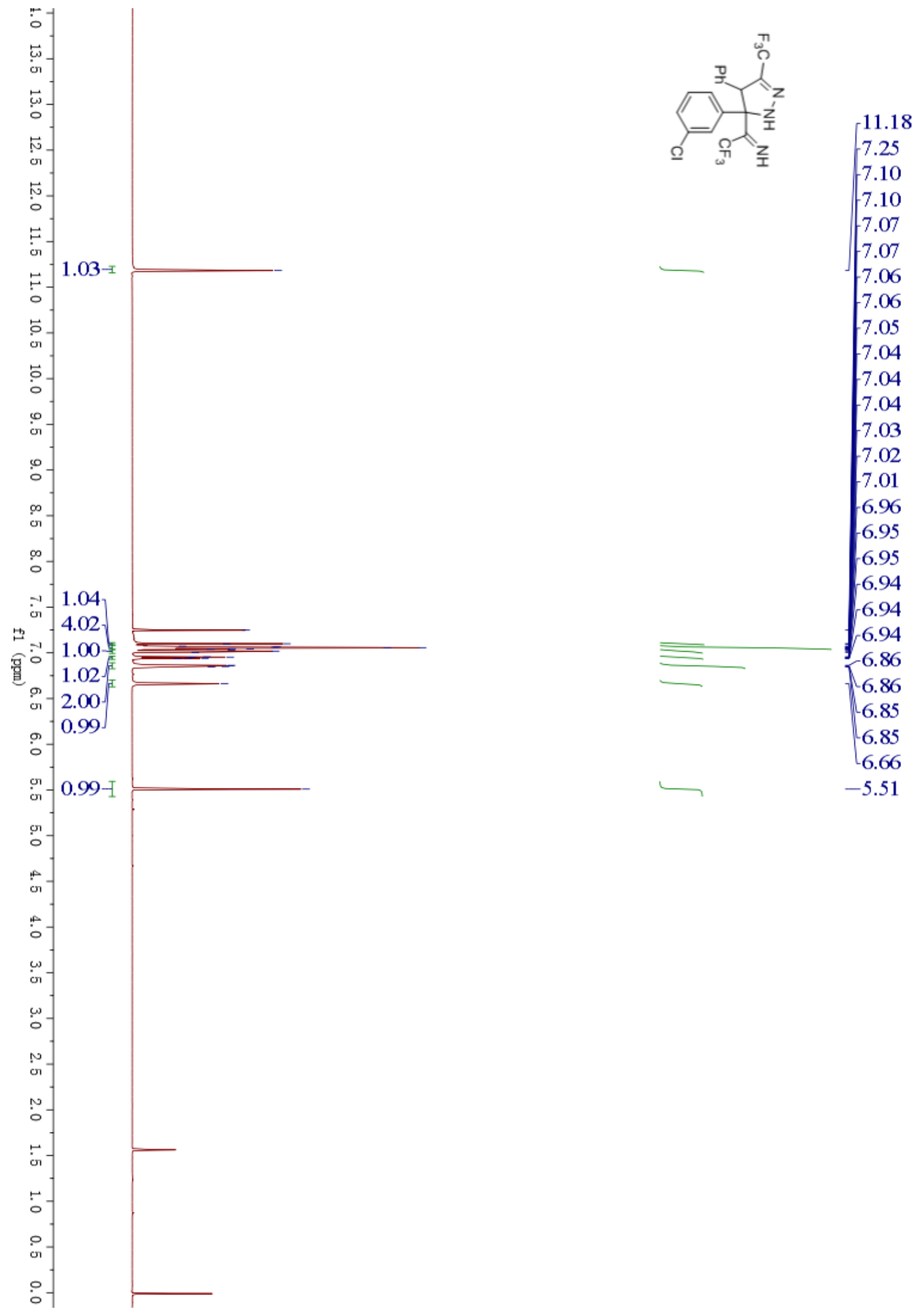
${ }^{13} \mathrm{C} \mathrm{NMR}\left(151 \mathrm{MHz}, \mathrm{CDCl}_{3}\right)$ spectrum of $\mathbf{2 h}$
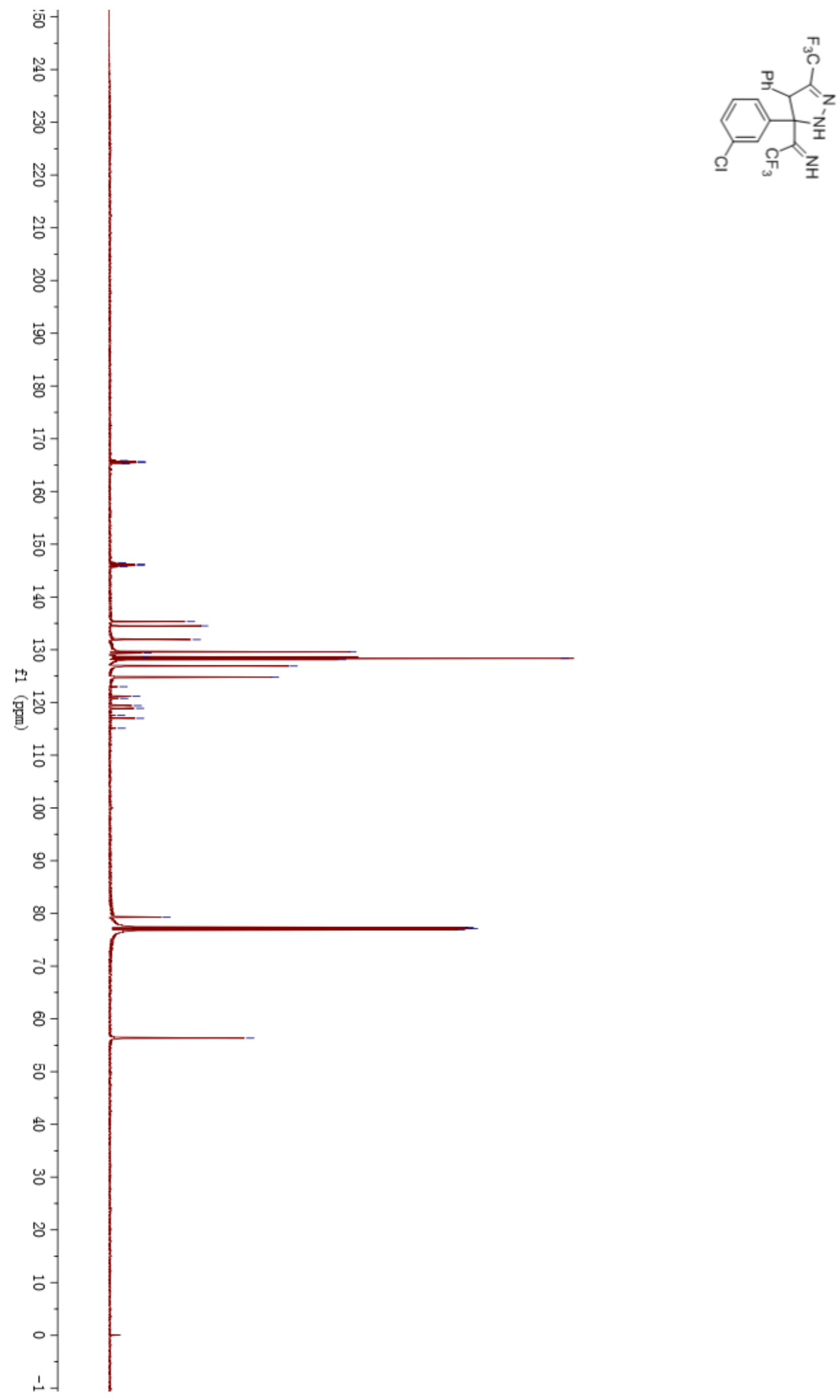
${ }^{19} \mathrm{~F} \mathrm{NMR}\left(565 \mathrm{MHz}, \mathrm{CDCl}_{3}\right)$ spectrum of $\mathbf{2 h}$

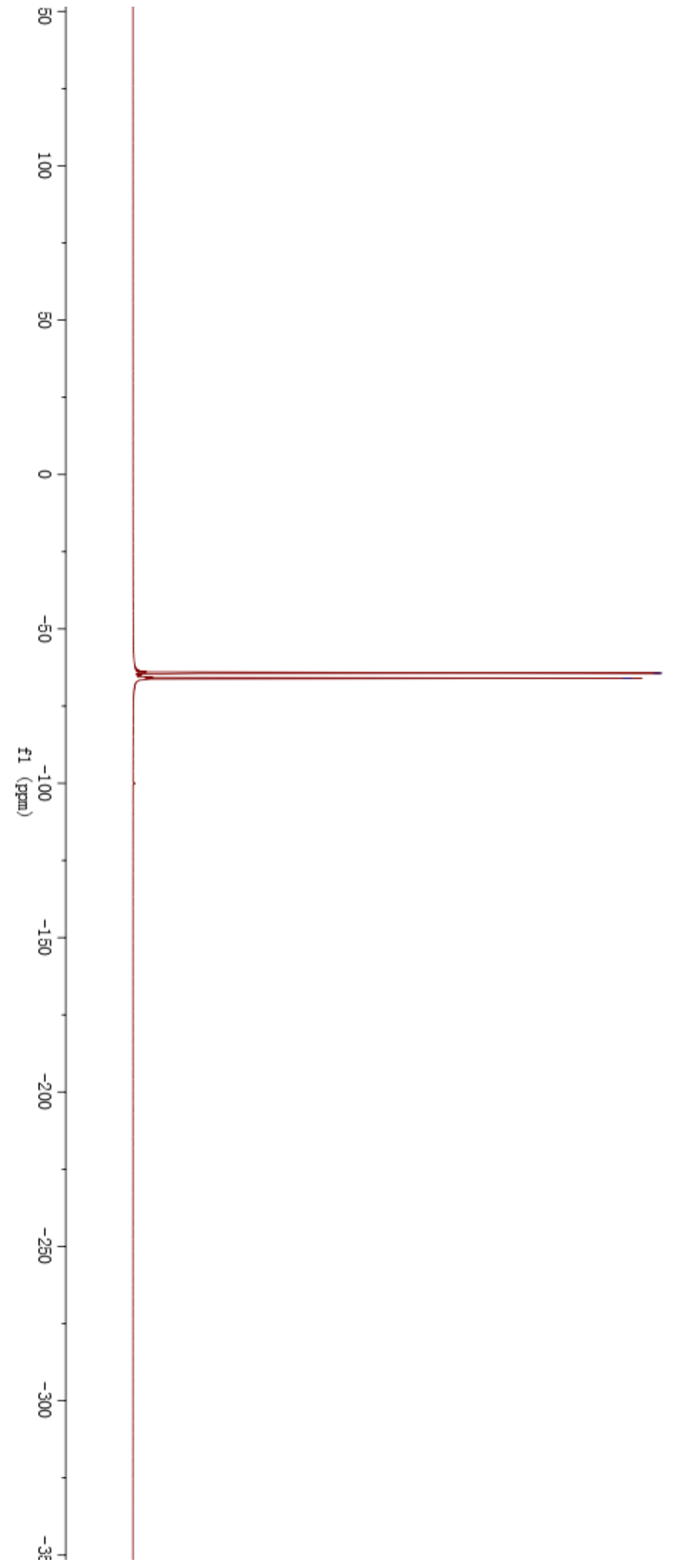


${ }^{1} \mathrm{H}$ NMR $\left(600 \mathrm{MHz}, \mathrm{CDCl}_{3}\right)$ spectrum of $\mathbf{2 i}$

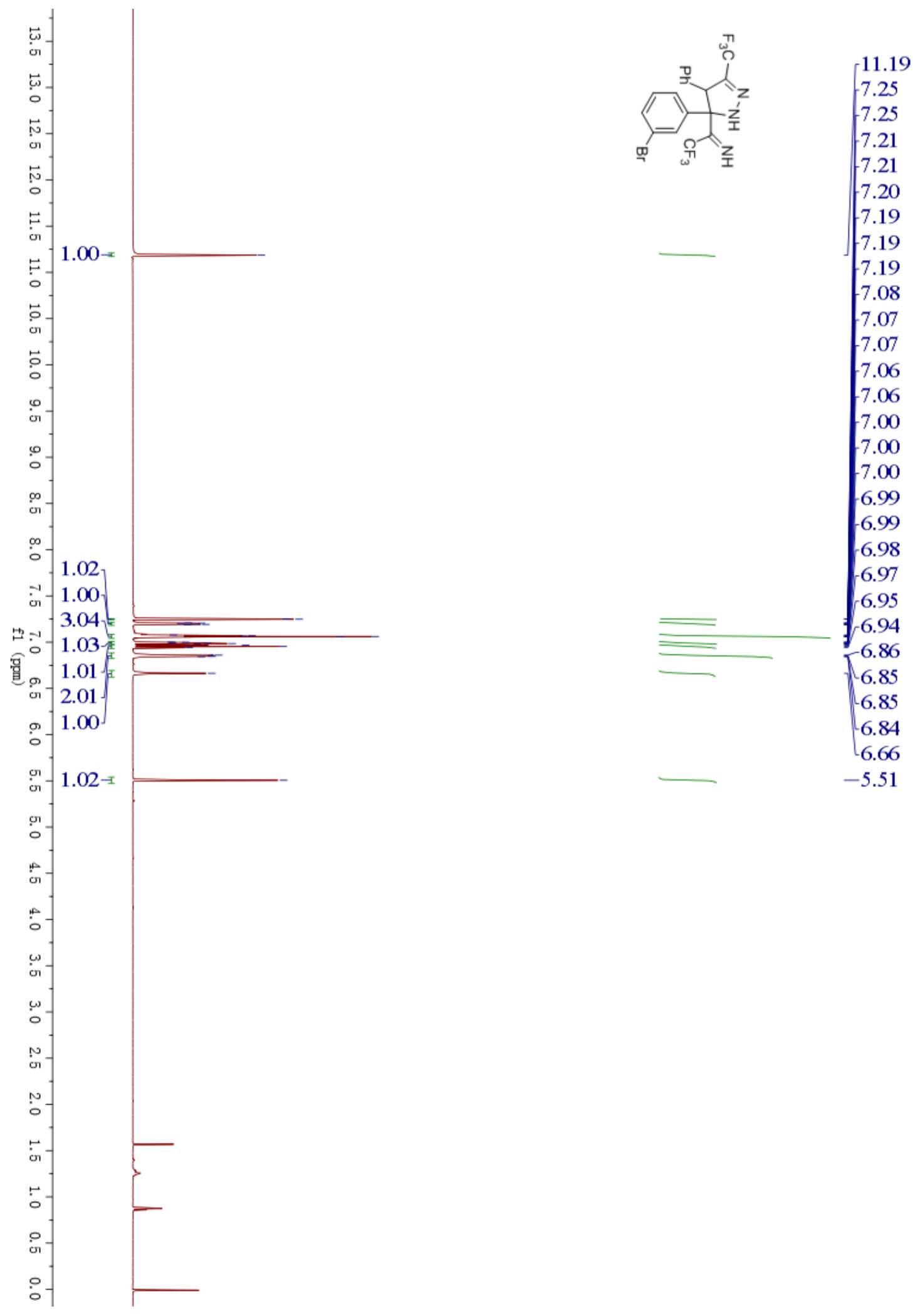


${ }^{13} \mathrm{C}$ NMR (151 MHz, $\left.\mathrm{CDCl}_{3}\right)$ spectrum of $\mathbf{2 i}$

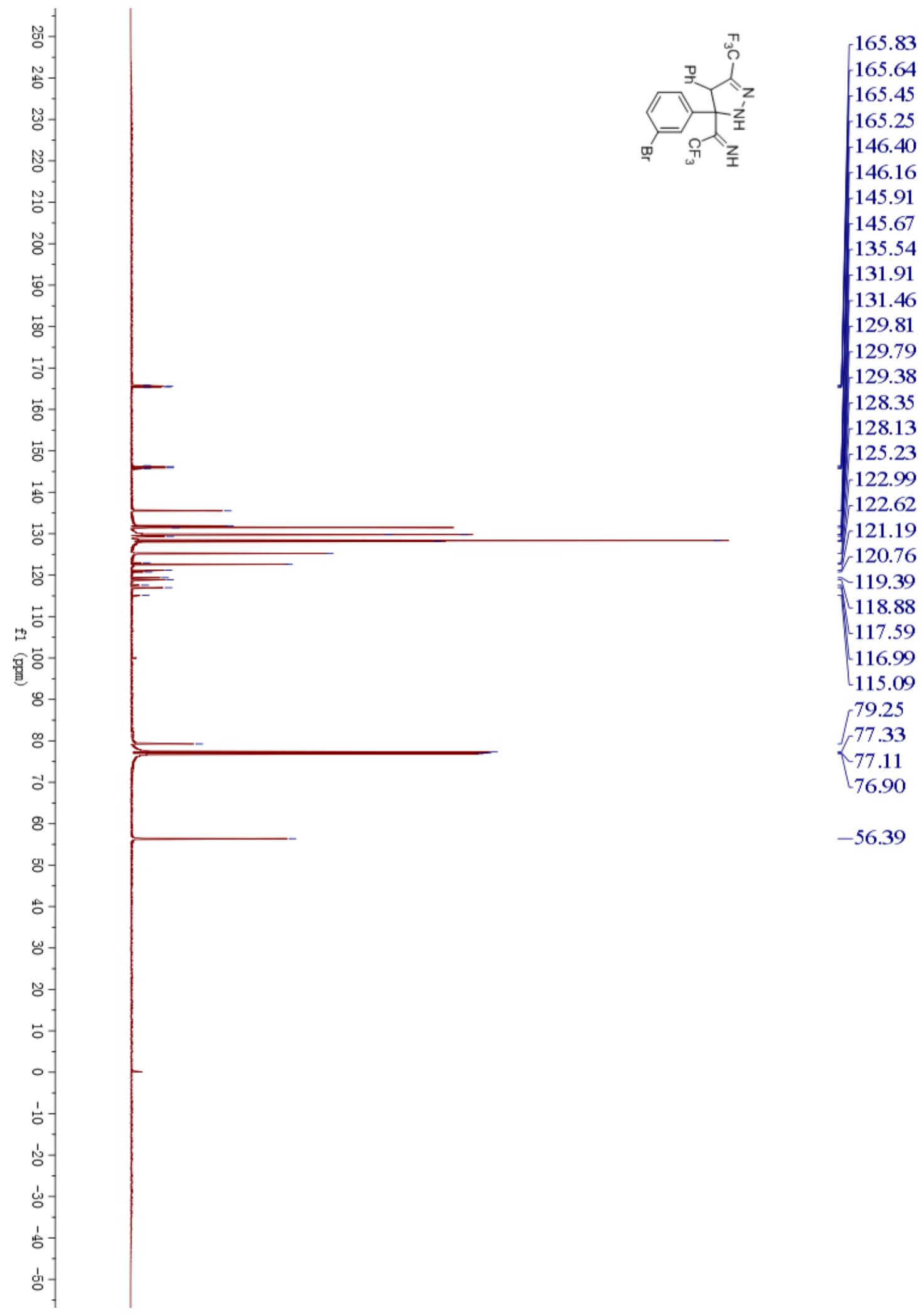


${ }^{19} \mathrm{~F}$ NMR (565 MHz, $\mathrm{CDCl}_{3}$ ) spectrum of $\mathbf{2 i}$

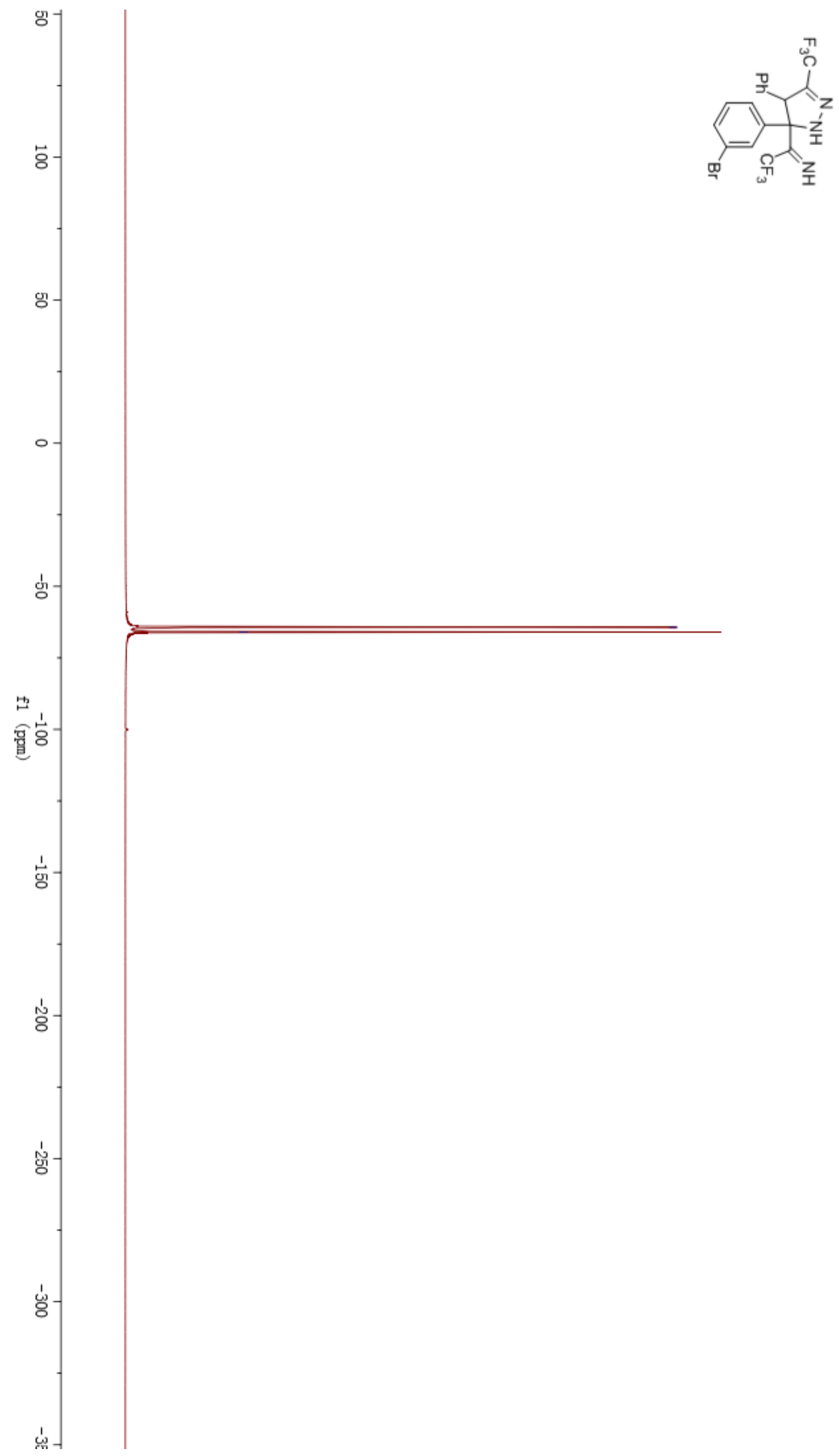

$-64.33$

-66.02 
${ }^{1} \mathrm{H}$ NMR $\left(600 \mathrm{MHz}, \mathrm{CDCl}_{3}\right)$ spectrum of $\mathbf{2 j}$
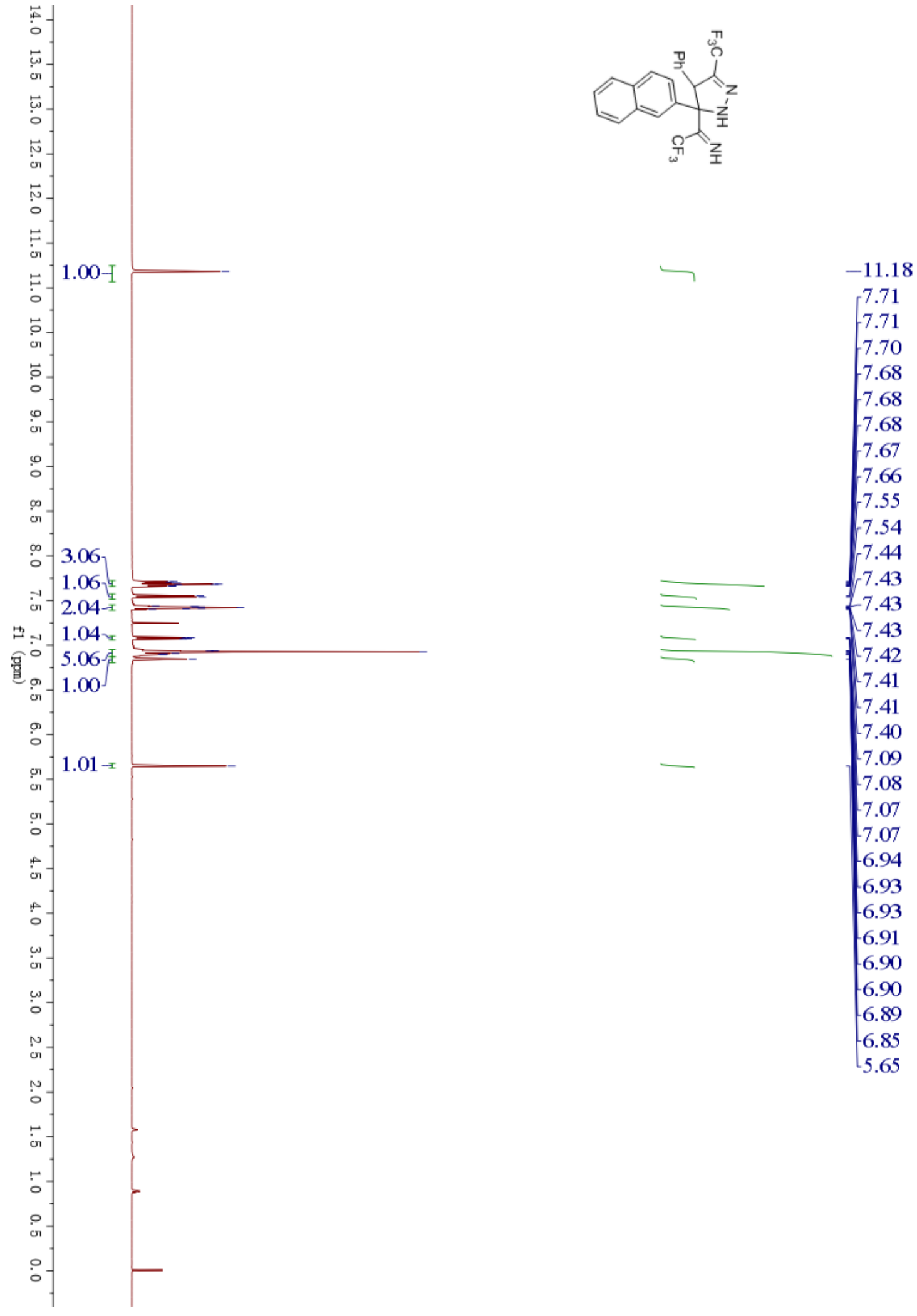
${ }^{13} \mathrm{C}$ NMR (151 MHz, $\mathrm{CDCl}_{3}$ ) spectrum of $\mathbf{2} \mathbf{j}$

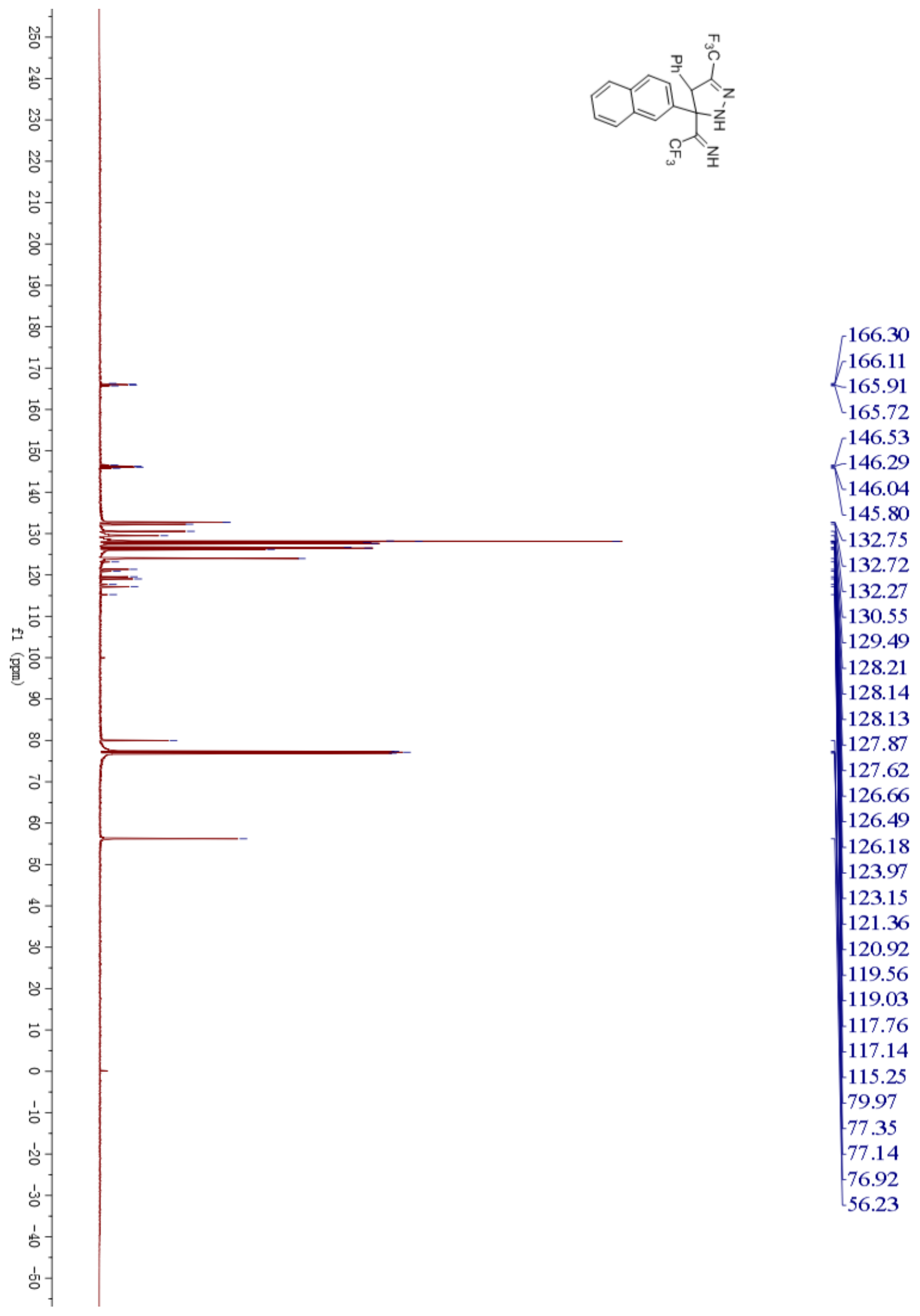


${ }^{19} \mathrm{~F}$ NMR (565 MHz, $\mathrm{CDCl}_{3}$ ) spectrum of $\mathbf{2} \mathbf{j}$

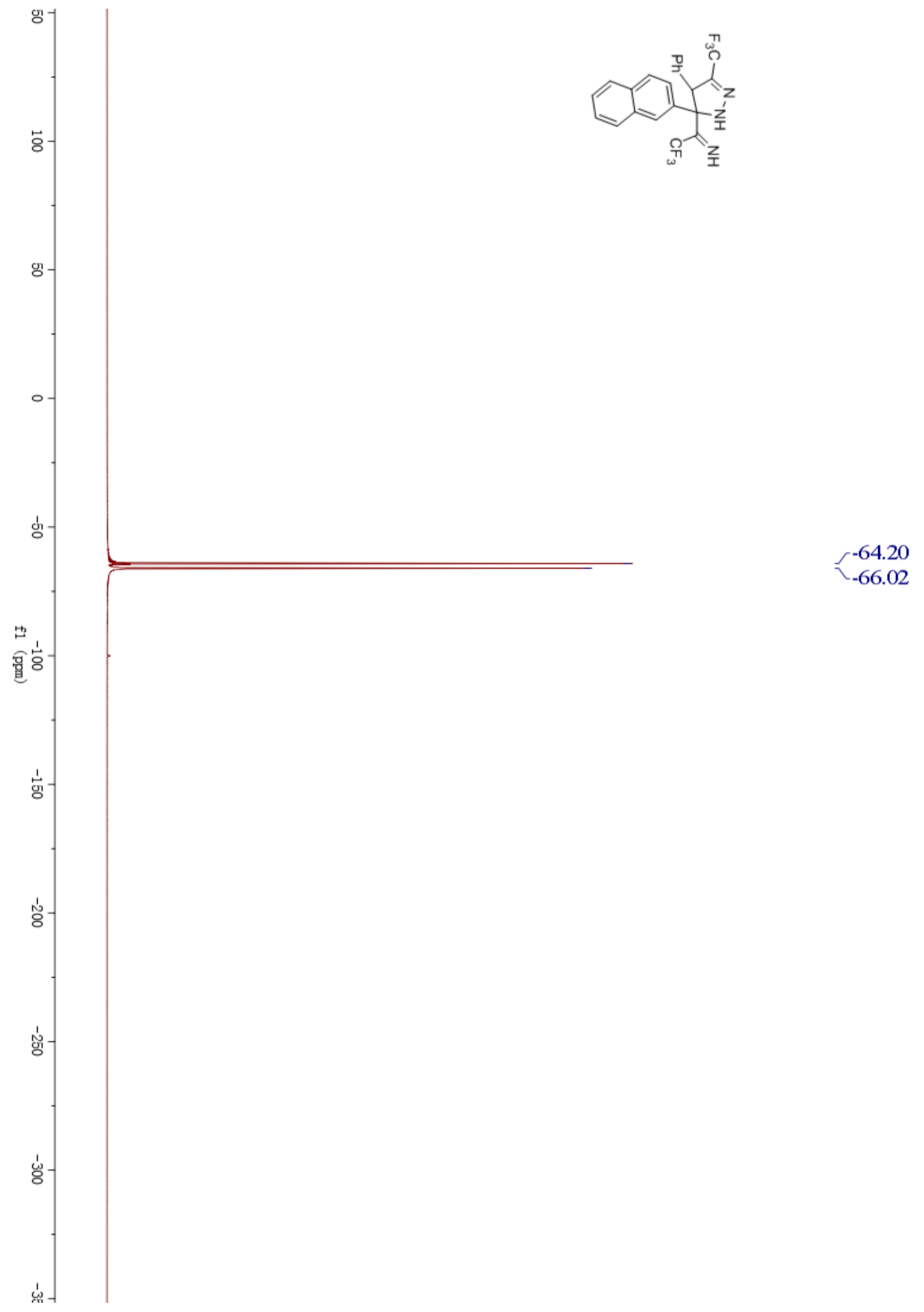


${ }^{1} \mathrm{H}$ NMR (600 MHz, $\left.\mathrm{CDCl}_{3}\right)$ spectrum of $\mathbf{2 k}$

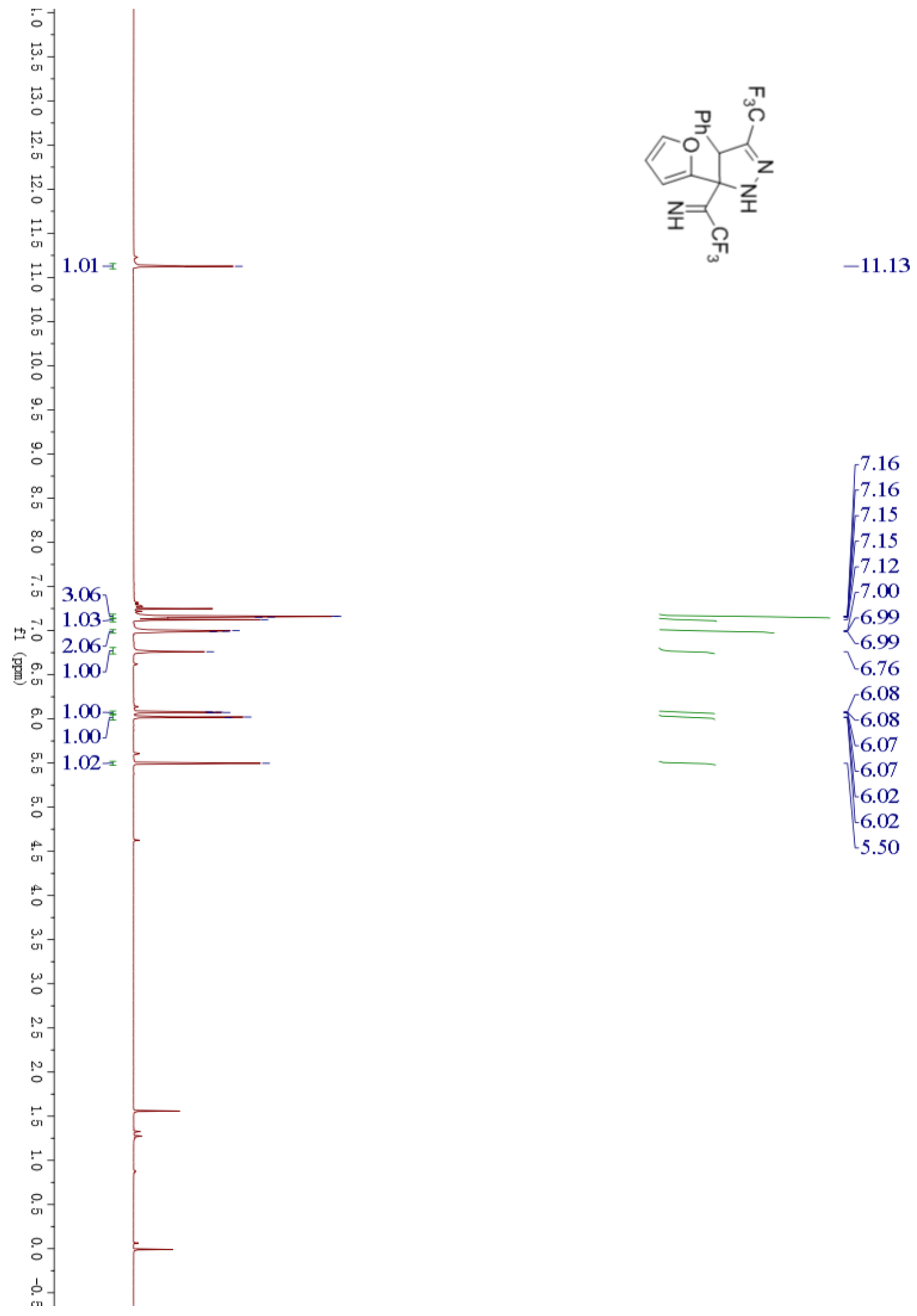


${ }^{13} \mathrm{C} \mathrm{NMR}\left(151 \mathrm{MHz}, \mathrm{CDCl}_{3}\right)$ spectrum of $\mathbf{2 k}$

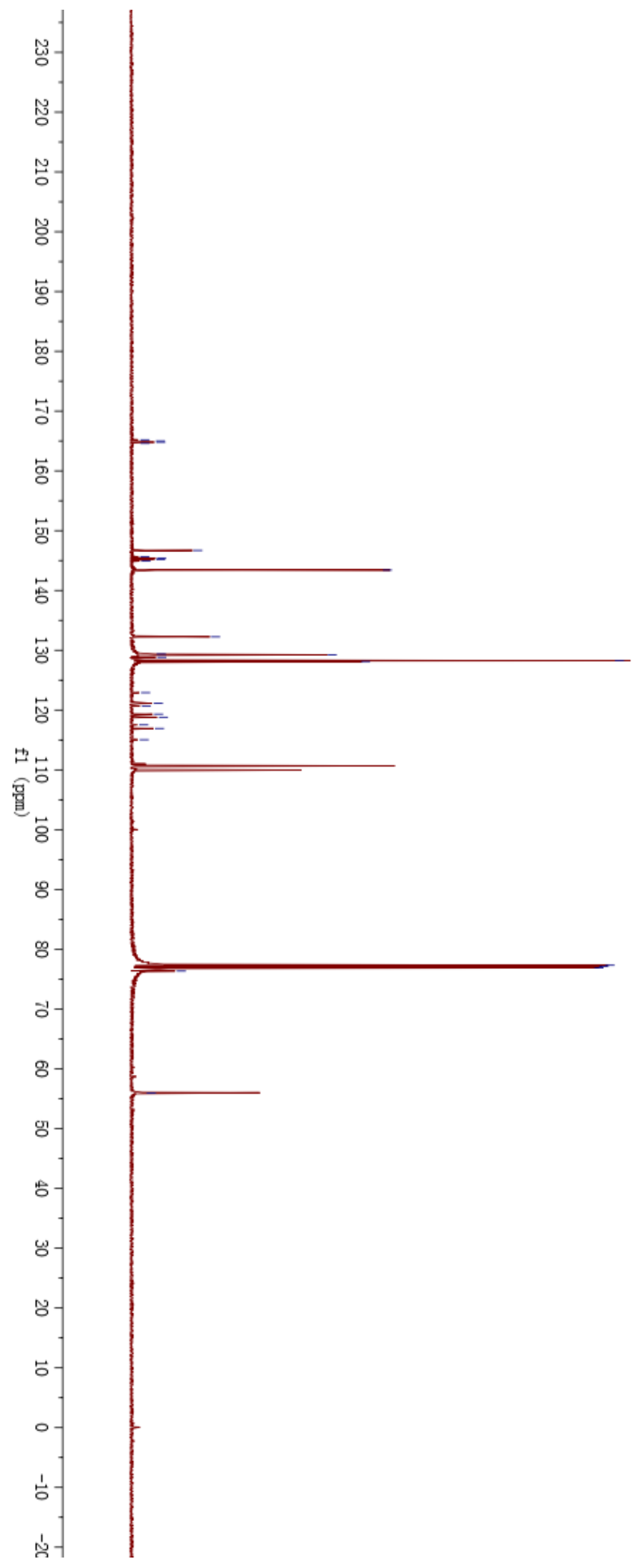

165.20

165.01 164.81 164.61 146.70 145.64 145.40 145.16 $-144.91$

$\backslash_{143.42}$ 132.25 $-129.37$ 129.25 128.84 128.26 $-128.13$ 122.92

121.12

120.70

$-119.32$

$-118.81$

$-117.53$

$-116.93$

115.05

77.32

77.11

76.90

76.35

55.97 
${ }^{19} \mathrm{~F}$ NMR $\left(565 \mathrm{MHz}, \mathrm{CDCl}_{3}\right)$ spectrum of $\mathbf{2 k}$

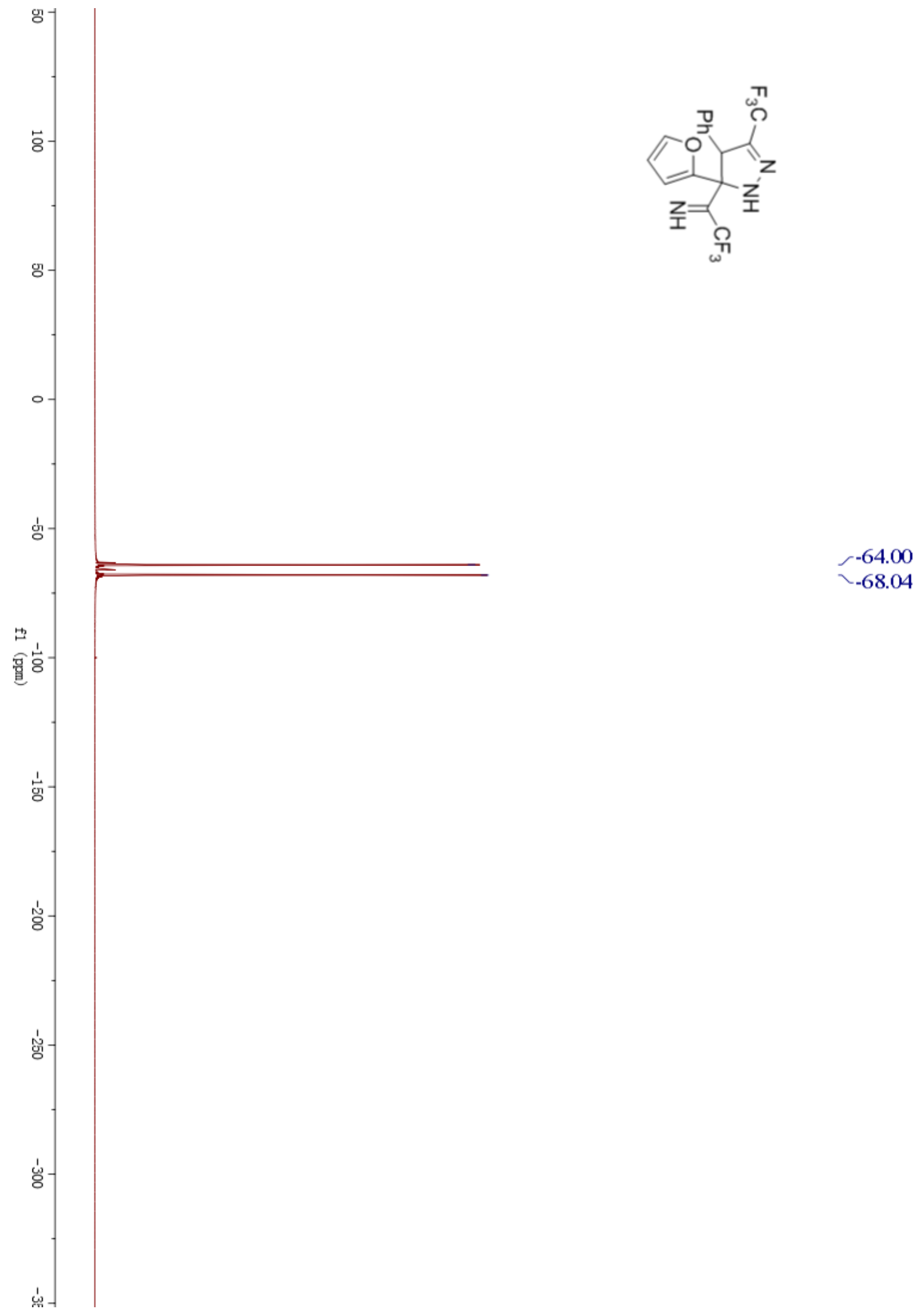


${ }^{1} \mathrm{H}$ NMR $\left(600 \mathrm{MHz}, \mathrm{CDCl}_{3}\right)$ spectrum of $\mathbf{2 l}$
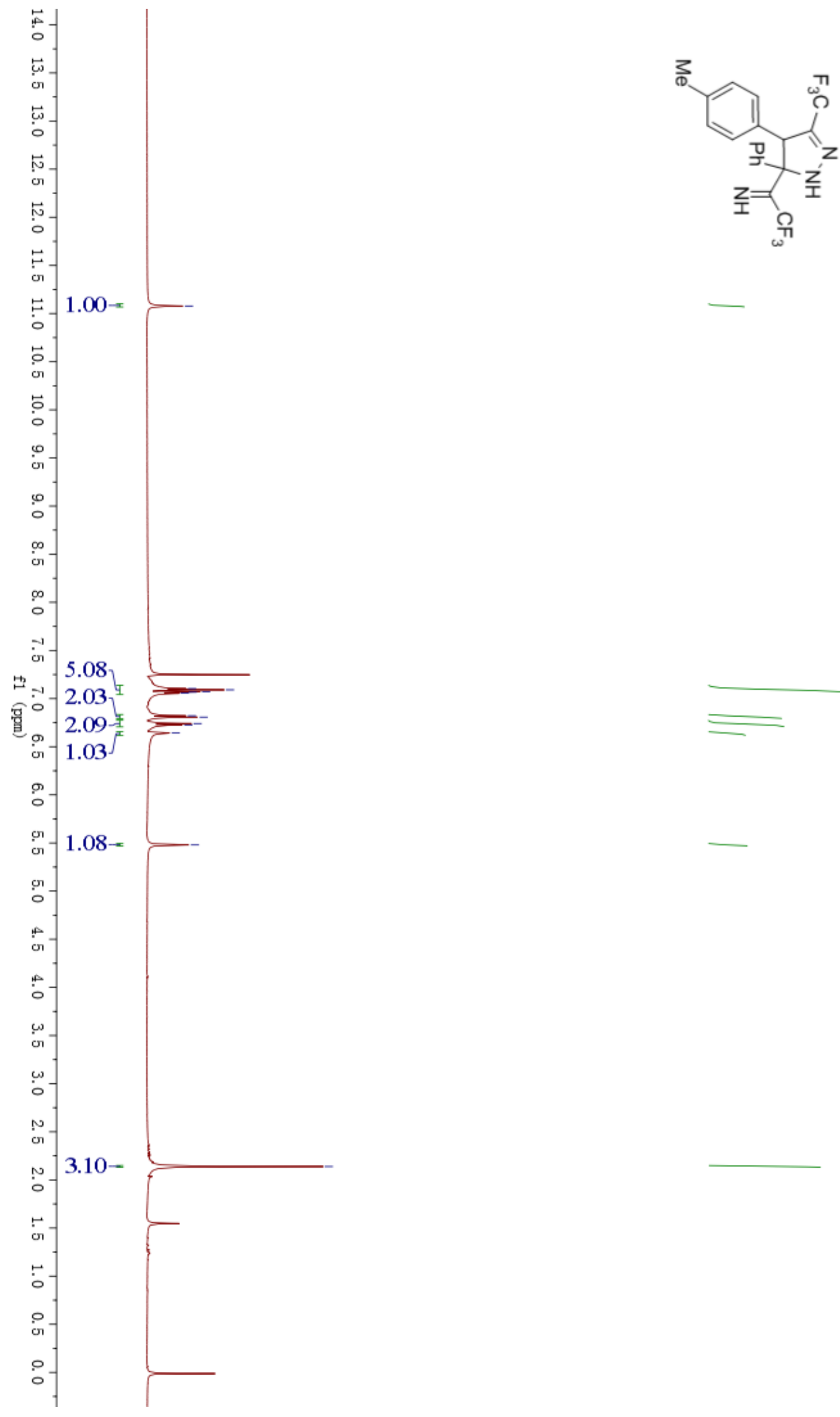

$-11.08$

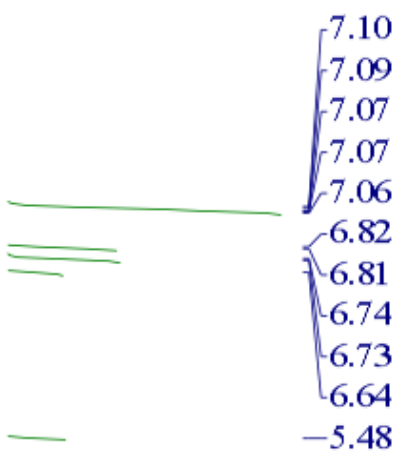

$-2.14$ 
${ }^{13} \mathrm{C} \mathrm{NMR}\left(151 \mathrm{MHz}, \mathrm{CDCl}_{3}\right)$ spectrum of $\mathbf{2 l}$
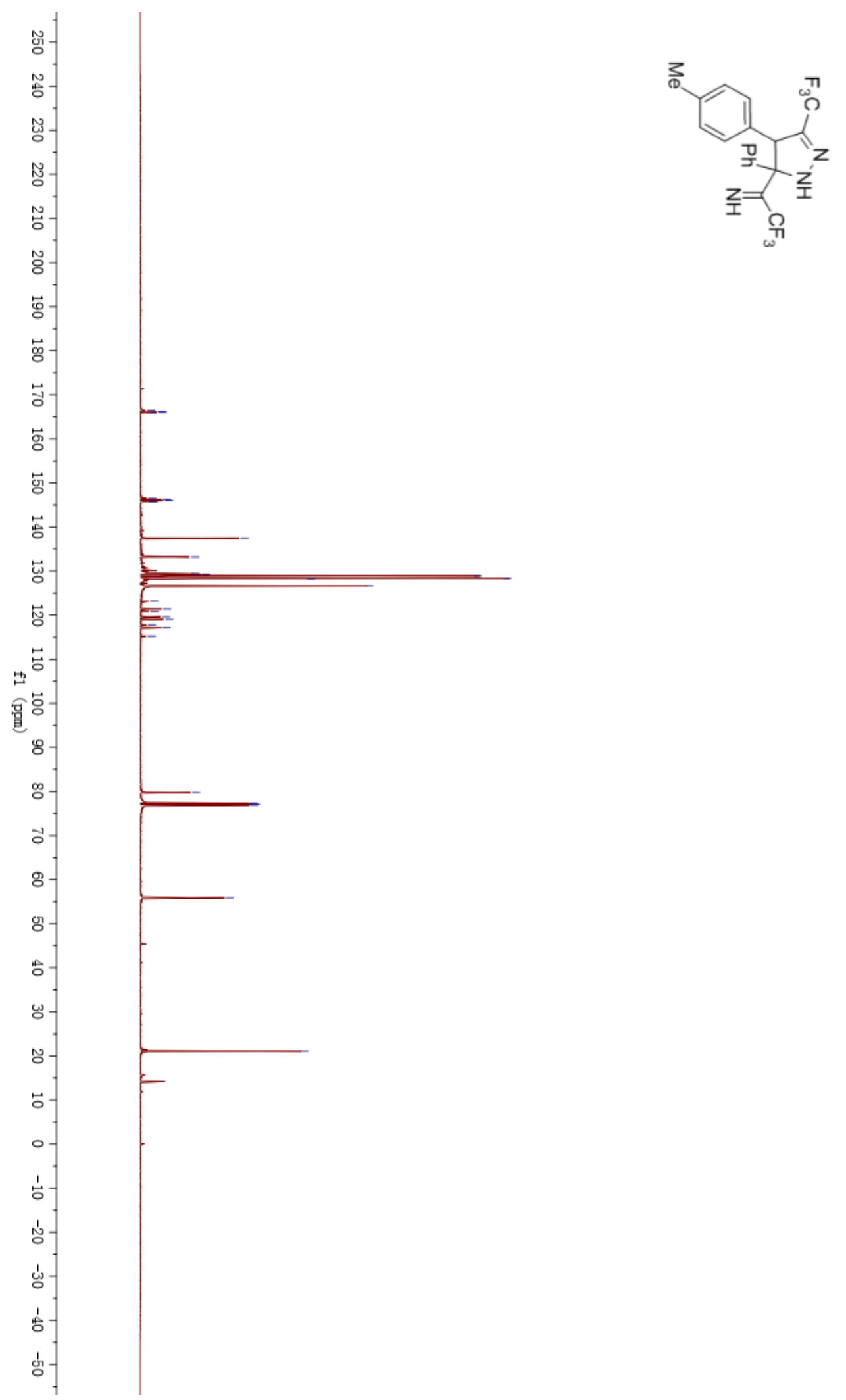

[166.43

166.23

$-166.04$

$-165.85$

$-146.47$

146.23

$-145.99$

$-145.75$

137.44

133.19

129.41

129.28

128.87

128.33

$-128.27$

126.64

123.20

121.40

120.92

119.61

119.03

$-117.81$

117.14

115.25

79.77

77.35

77.14

76.92

$-55.87$

$-21.06$ 
${ }^{19} \mathrm{~F}$ NMR (565 MHz, $\mathrm{CDCl}_{3}$ ) spectrum of $\mathbf{2 l}$
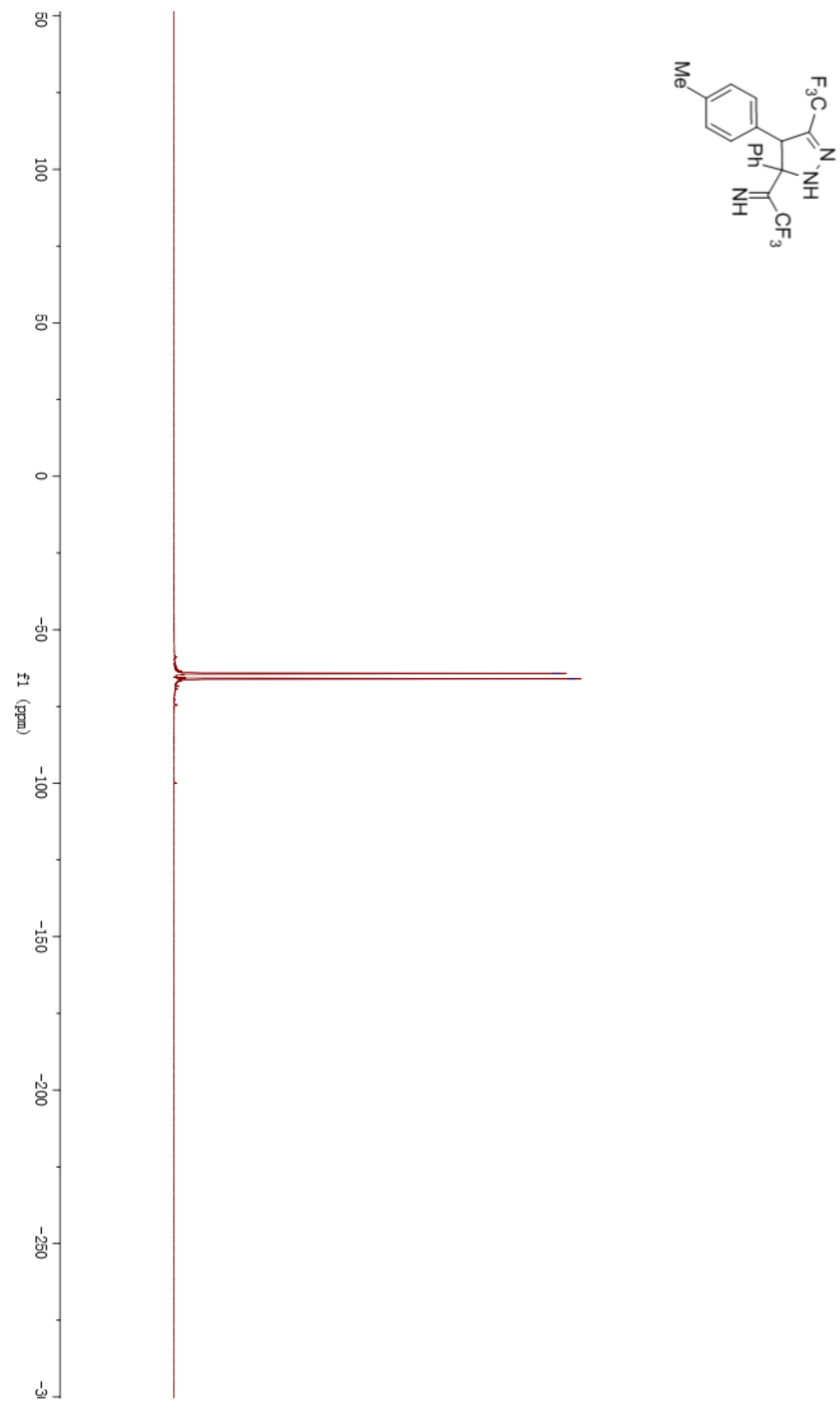
${ }^{1} \mathrm{H}$ NMR (600 MHz, $\mathrm{CDCl}_{3}$ ) spectrum of $\mathbf{2 m}$
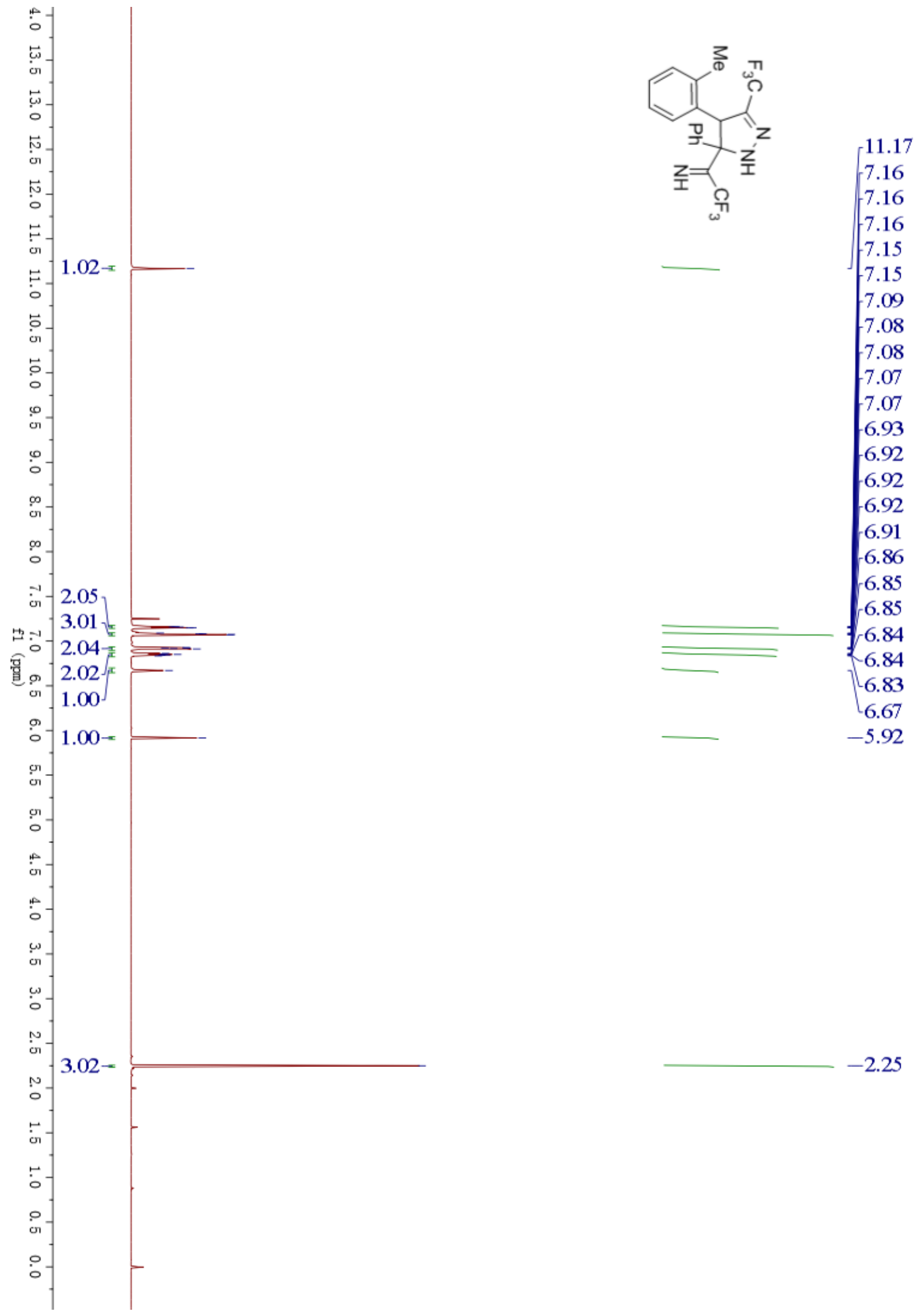
${ }^{13} \mathrm{C} \mathrm{NMR}\left(151 \mathrm{MHz}, \mathrm{CDCl}_{3}\right)$ spectrum of $\mathbf{2 m}$

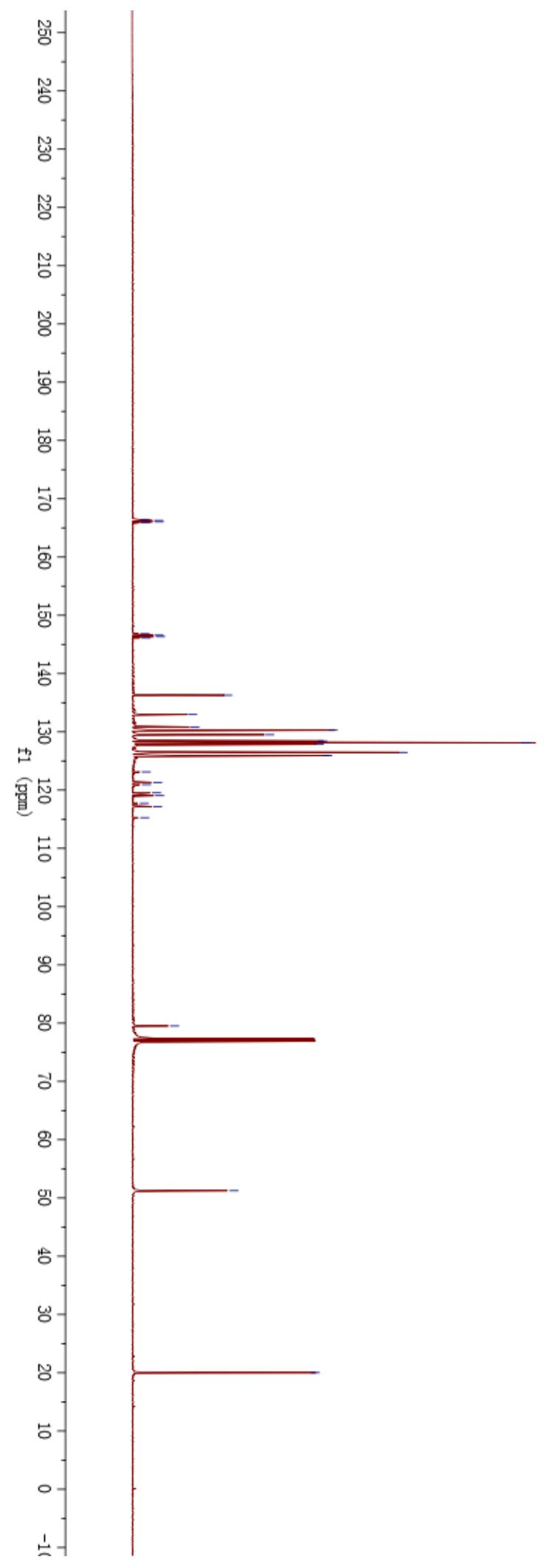

166.45

$-166.26$

$-166.07$

165.88

$-146.83$

146.58

146.34

$-146.10$

136.29

$-132.97$

130.83

$-130.28$

129.50

128.43

128.13

127.85

126.46

125.92

123.11

121.32

$-120.94$

$-119.52$

$-119.05$

$-117.73$

$-117.16$

115.27

$-79.51$

$-51.24$

$-20.02$ 
${ }^{19} \mathrm{~F}$ NMR $\left(565 \mathrm{MHz}, \mathrm{CDCl}_{3}\right)$ spectrum of $\mathbf{2 m}$

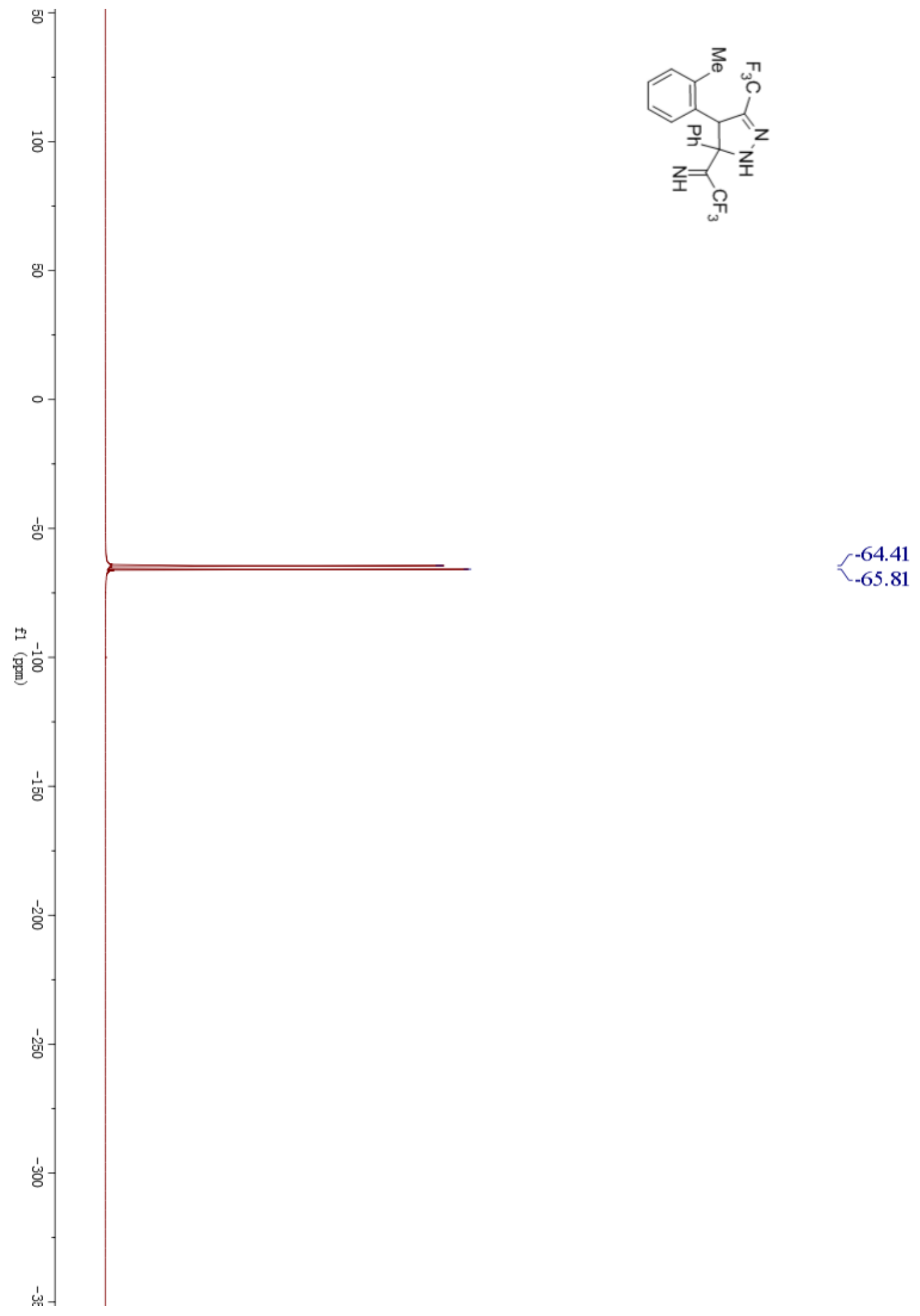


${ }^{1} \mathrm{H} \mathrm{NMR}\left(600 \mathrm{MHz}, \mathrm{CDCl}_{3}\right)$ spectrum of $\mathbf{2 n}$
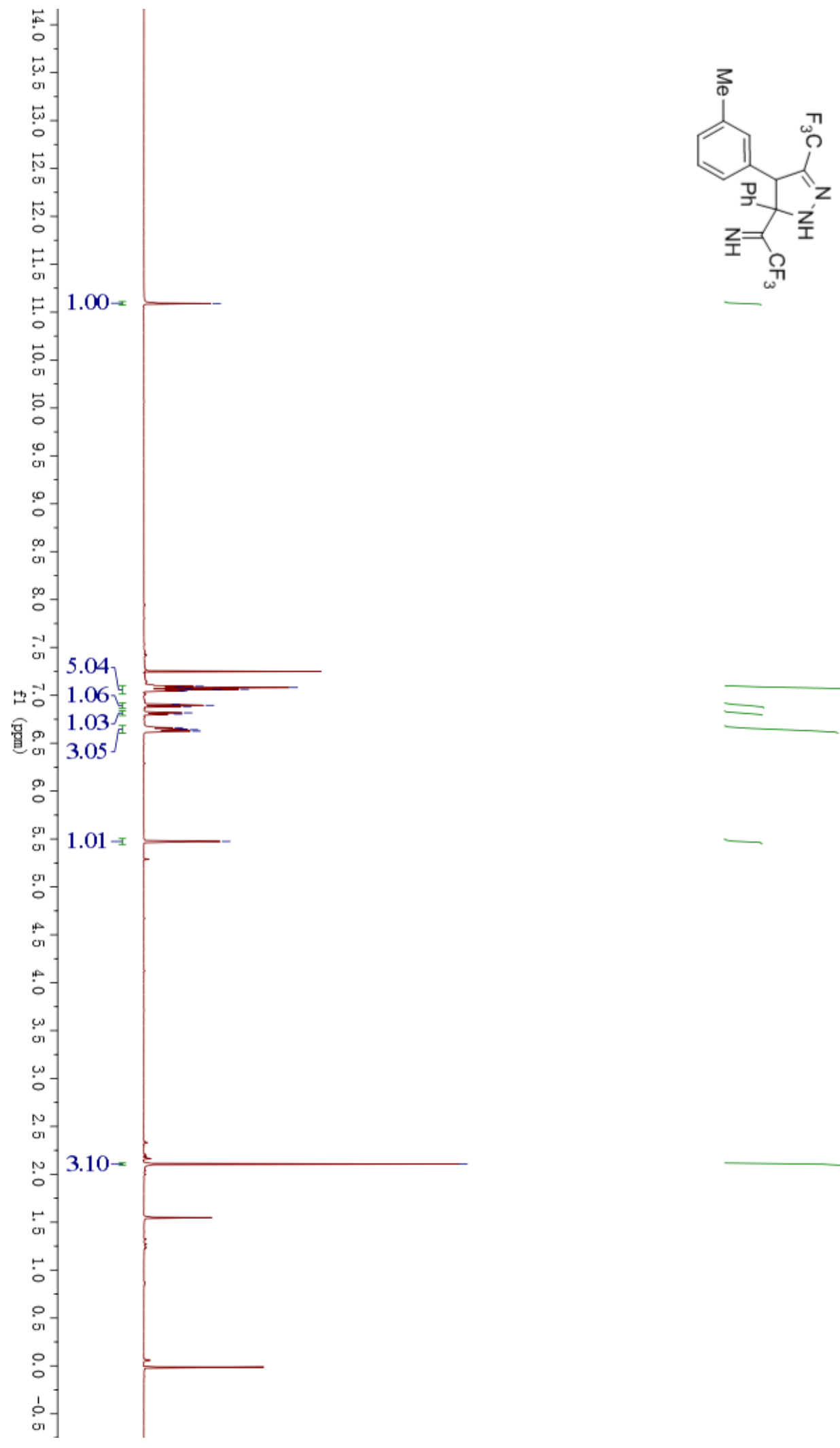

$-11.09$

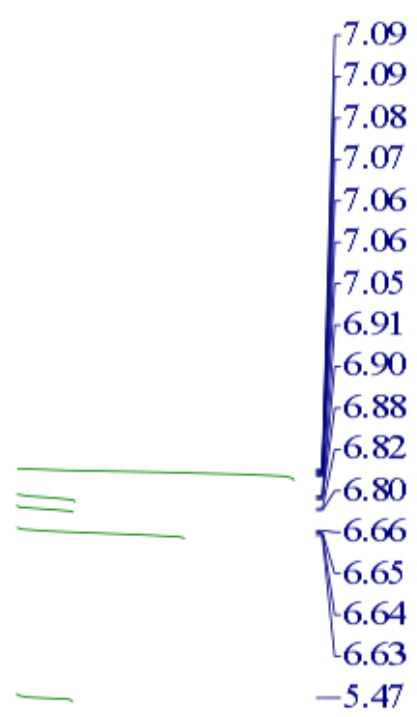

$-2.11$ 
${ }^{13} \mathrm{C} \mathrm{NMR}\left(151 \mathrm{MHz}, \mathrm{CDCl}_{3}\right)$ spectrum of $\mathbf{2 n}$

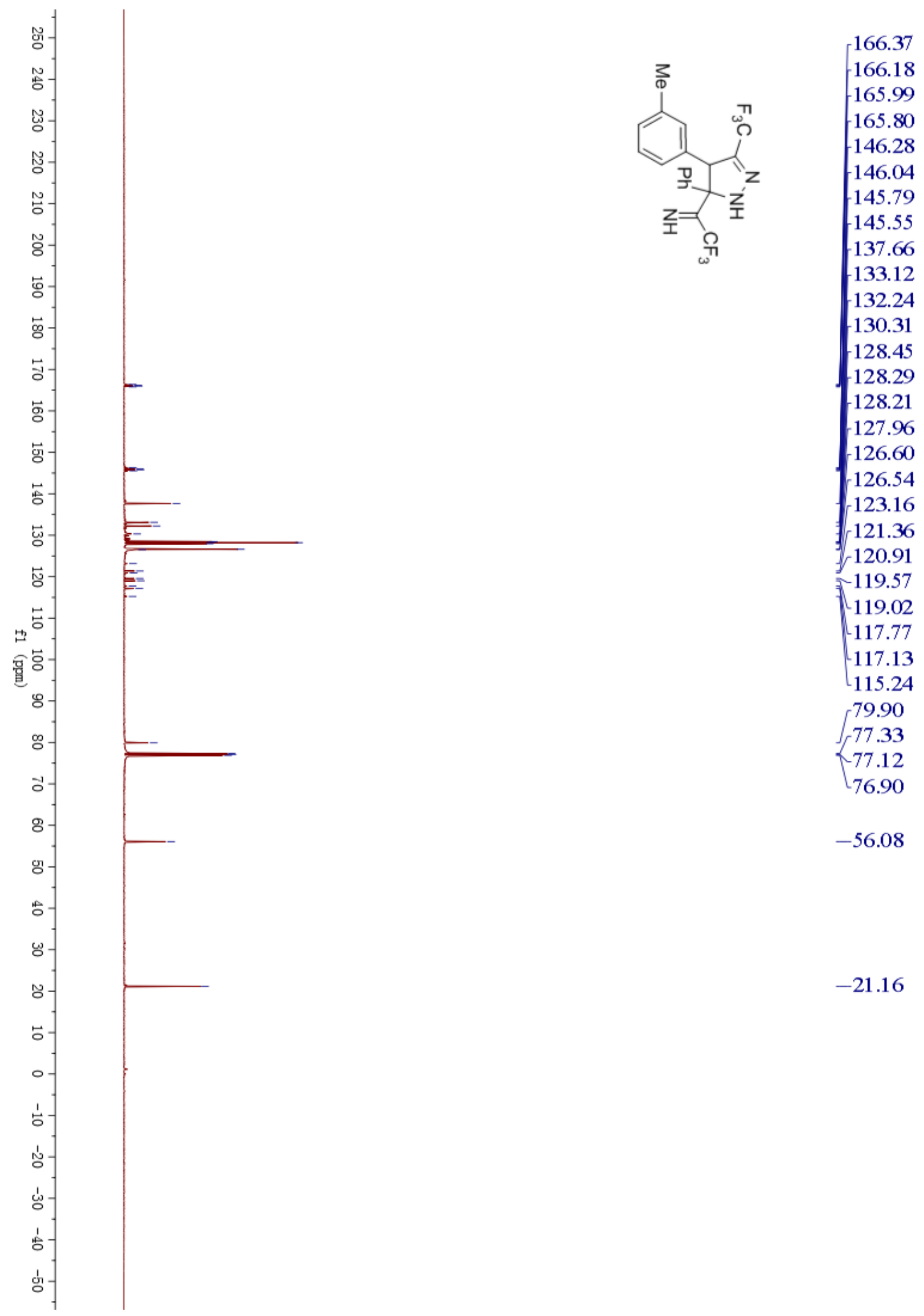


$\left.{ }^{19} \mathrm{~F} \mathrm{NMR} \mathrm{(565} \mathrm{MHz,} \mathrm{CDCl}_{3}\right)$ spectrum of $\mathbf{2 n}$
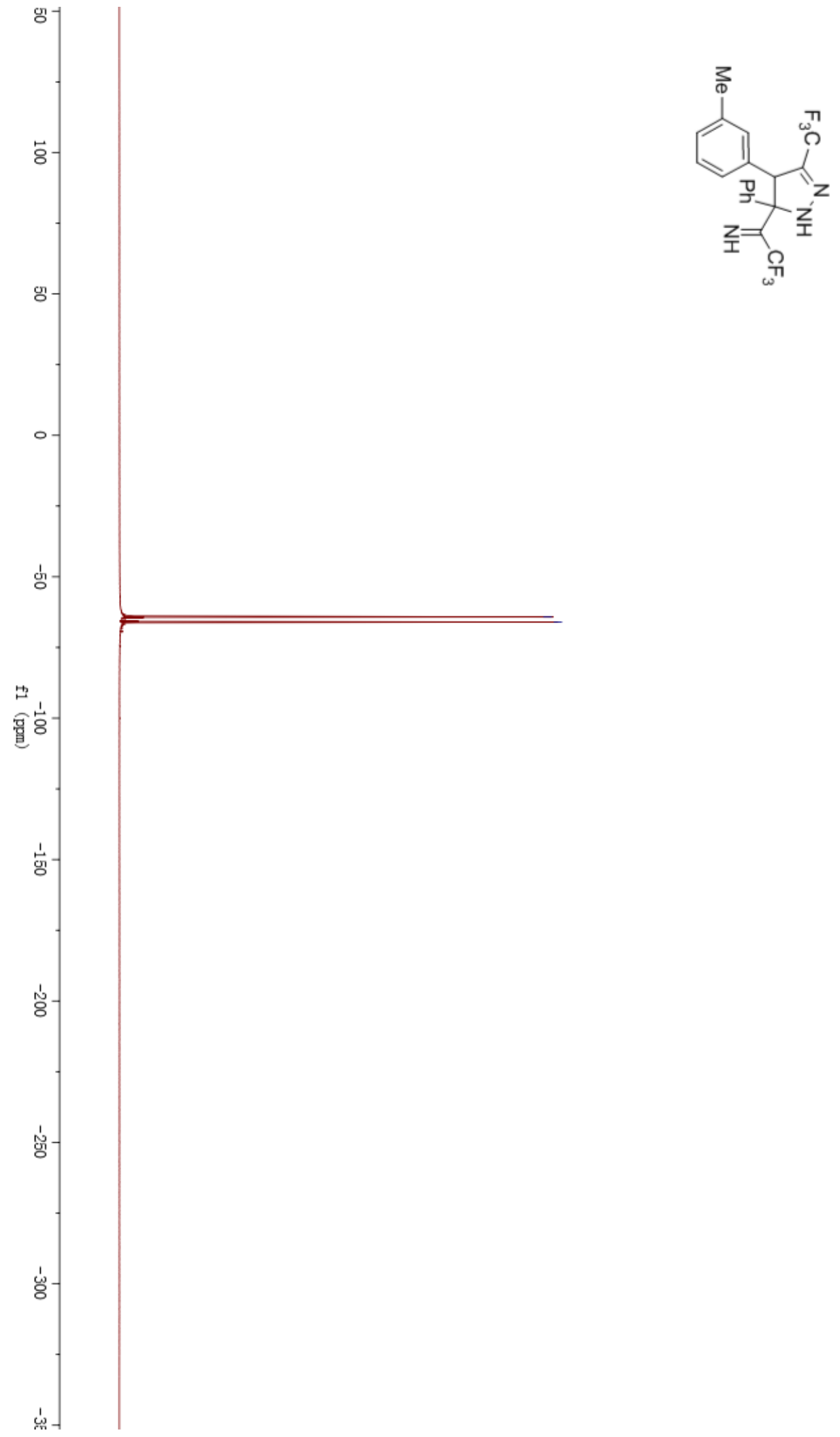

$-64.23$

-66.00 
${ }^{1} \mathrm{H} \mathrm{NMR}\left(600 \mathrm{MHz}, \mathrm{CDCl}_{3}\right)$ spectrum of $\mathbf{2 o}$
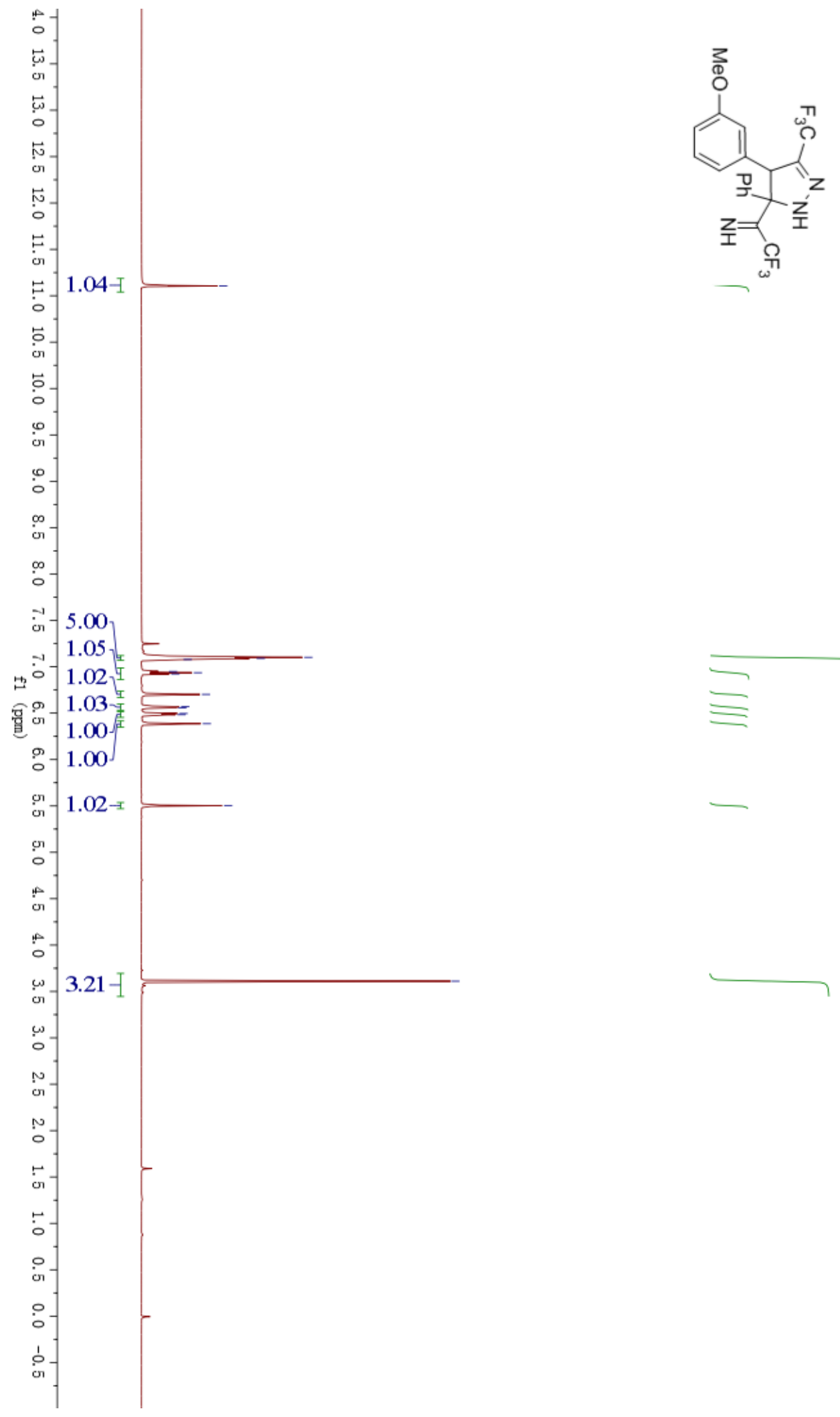

$-11.11$

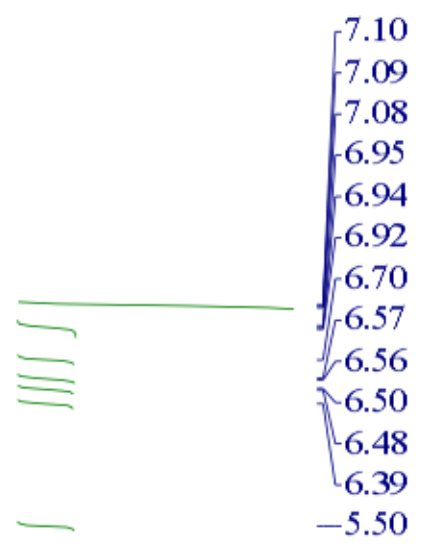

$-3.61$ 
${ }^{13} \mathrm{C} \mathrm{NMR}\left(151 \mathrm{MHz}, \mathrm{CDCl}_{3}\right)$ spectrum of $2 \mathrm{o}$
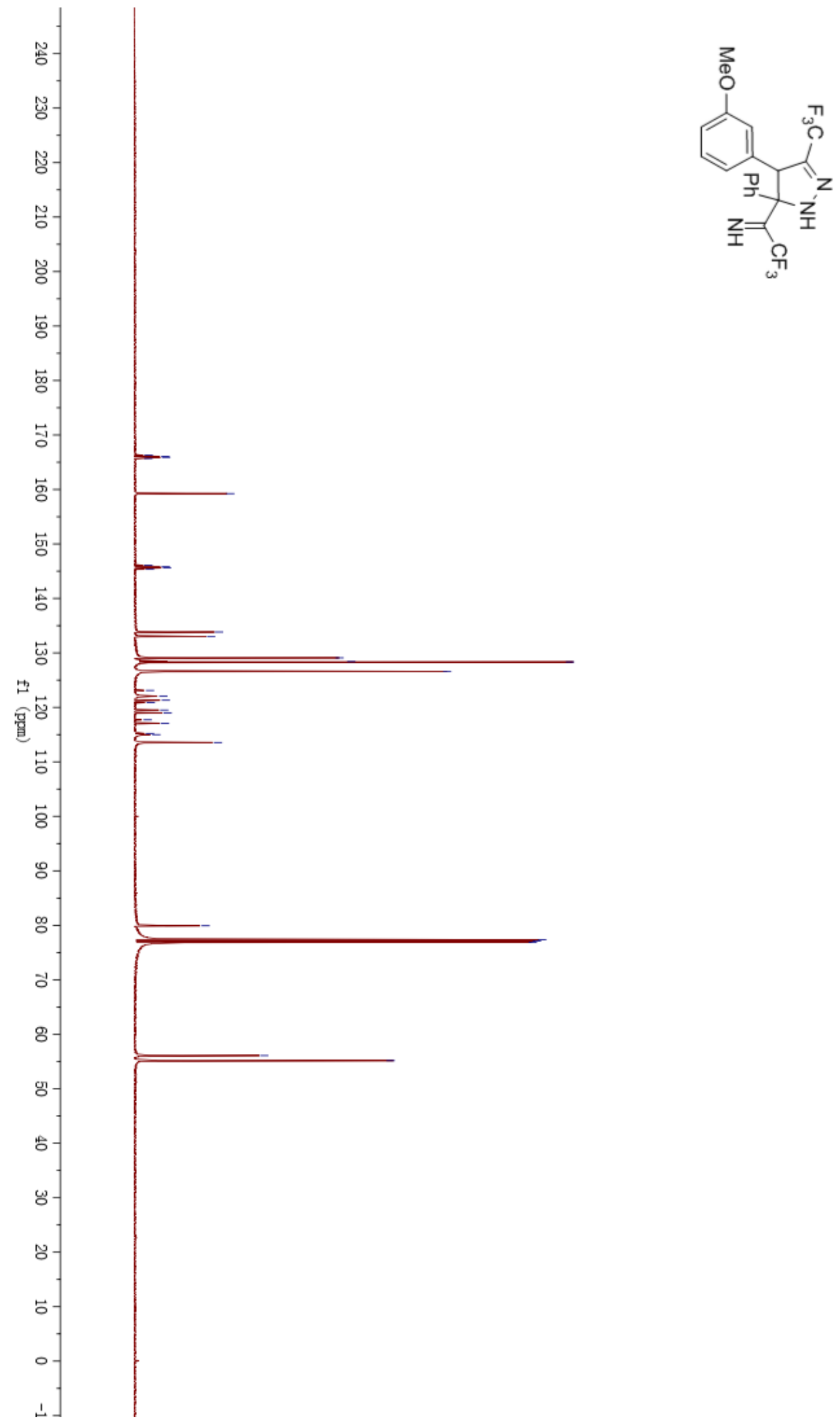

166.27

165.70

159.24

146.08

$-145.83$

145.59

$-145.35$

133.84

133.00

129.09

128.40

$-128.35$

$-126.59$

$-123.11$

$-122.06$

$-121.31$

120.88

119.51

118.99

117.72

$-117.10$

$-115.21$

115.00

113.55

79.92

77.33

$-77.12$

$-76.91$

$-56.09$

55.16 
${ }^{19} \mathrm{~F}$ NMR $\left(565 \mathrm{MHz}, \mathrm{CDCl}_{3}\right)$ spectrum of $\mathbf{2 0}$

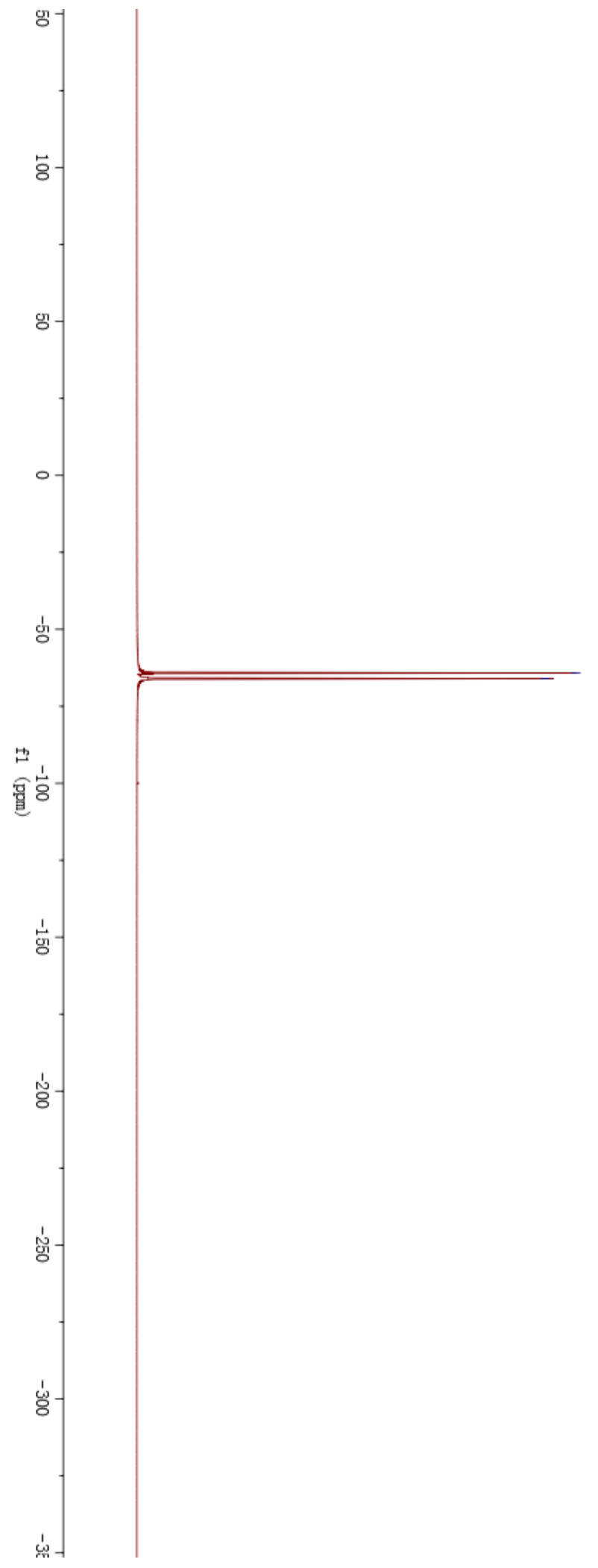

$-64.18$

ר.66.02 
${ }^{1} \mathrm{H}$ NMR (600 MHz, $\mathrm{CDCl}_{3}$ ) spectrum of $\mathbf{2 p}$

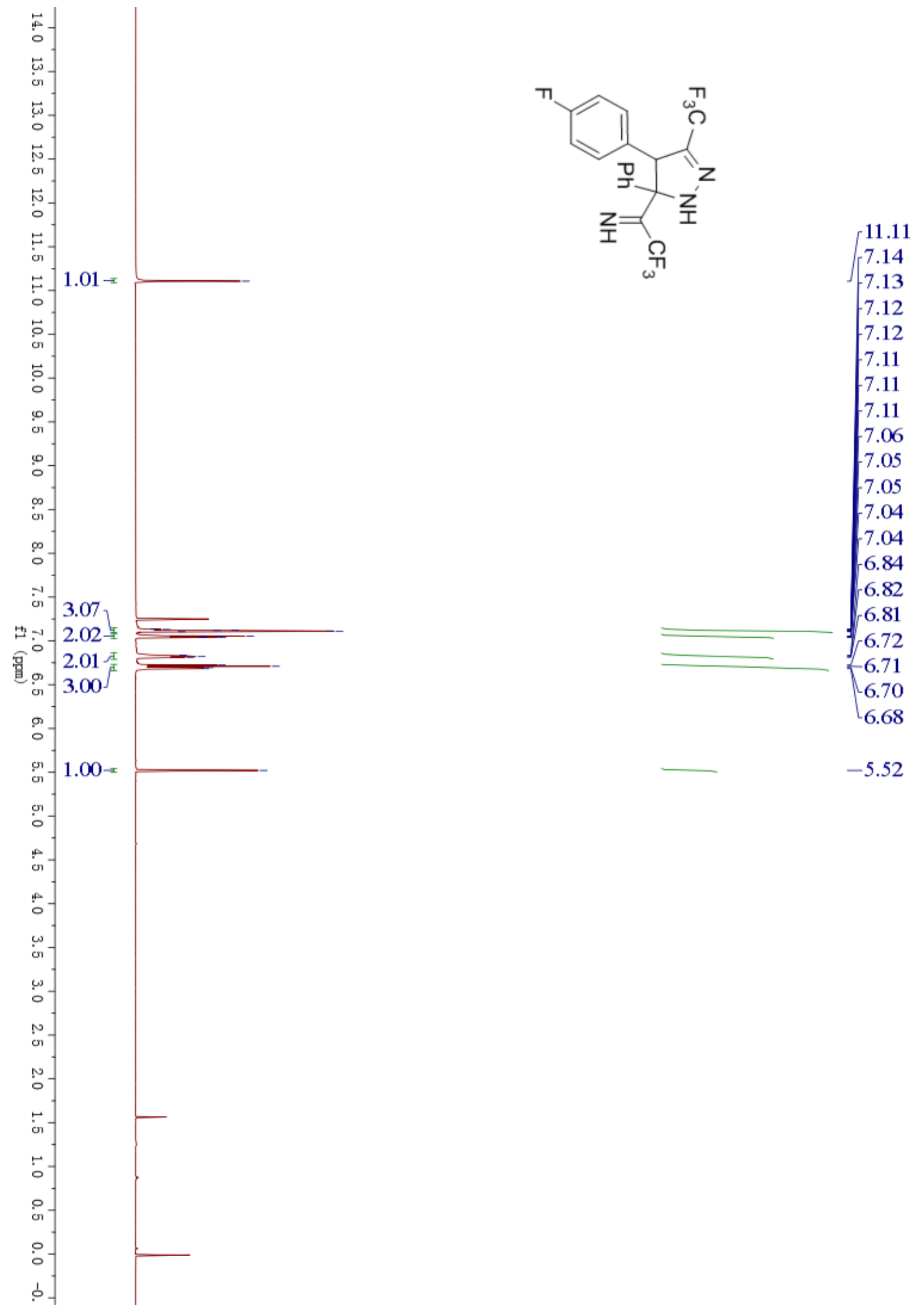


${ }^{13} \mathrm{C}$ NMR (151 MHz, $\left.\mathrm{CDCl}_{3}\right)$ spectrum of $\mathbf{2 p}$

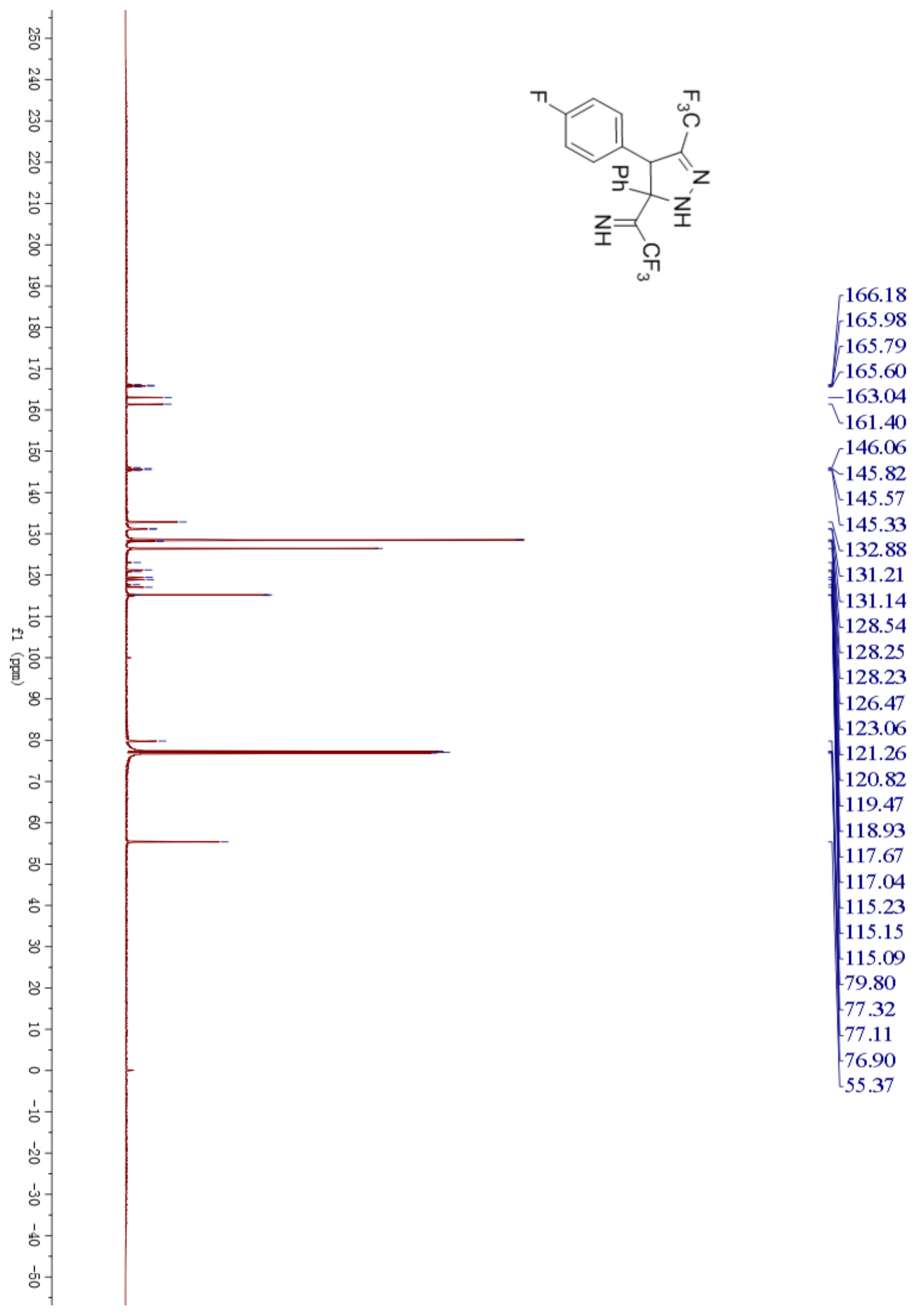


${ }^{19} \mathrm{~F} \mathrm{NMR}\left(565 \mathrm{MHz}, \mathrm{CDCl}_{3}\right)$ spectrum of $\mathbf{2 p}$

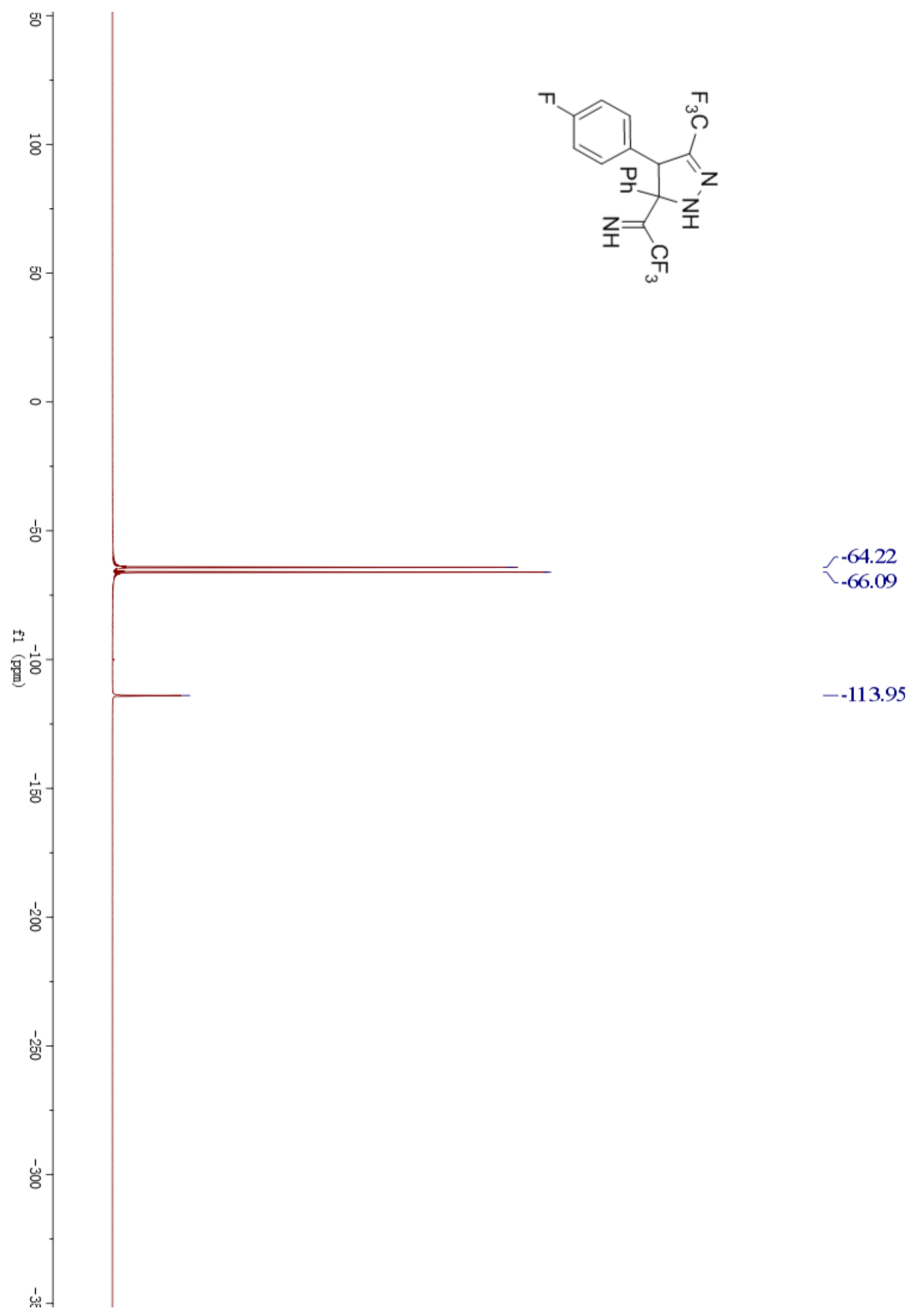


${ }^{1} \mathrm{H}$ NMR (600 MHz, $\mathrm{CDCl}_{3}$ ) spectrum of $\mathbf{2 q}$
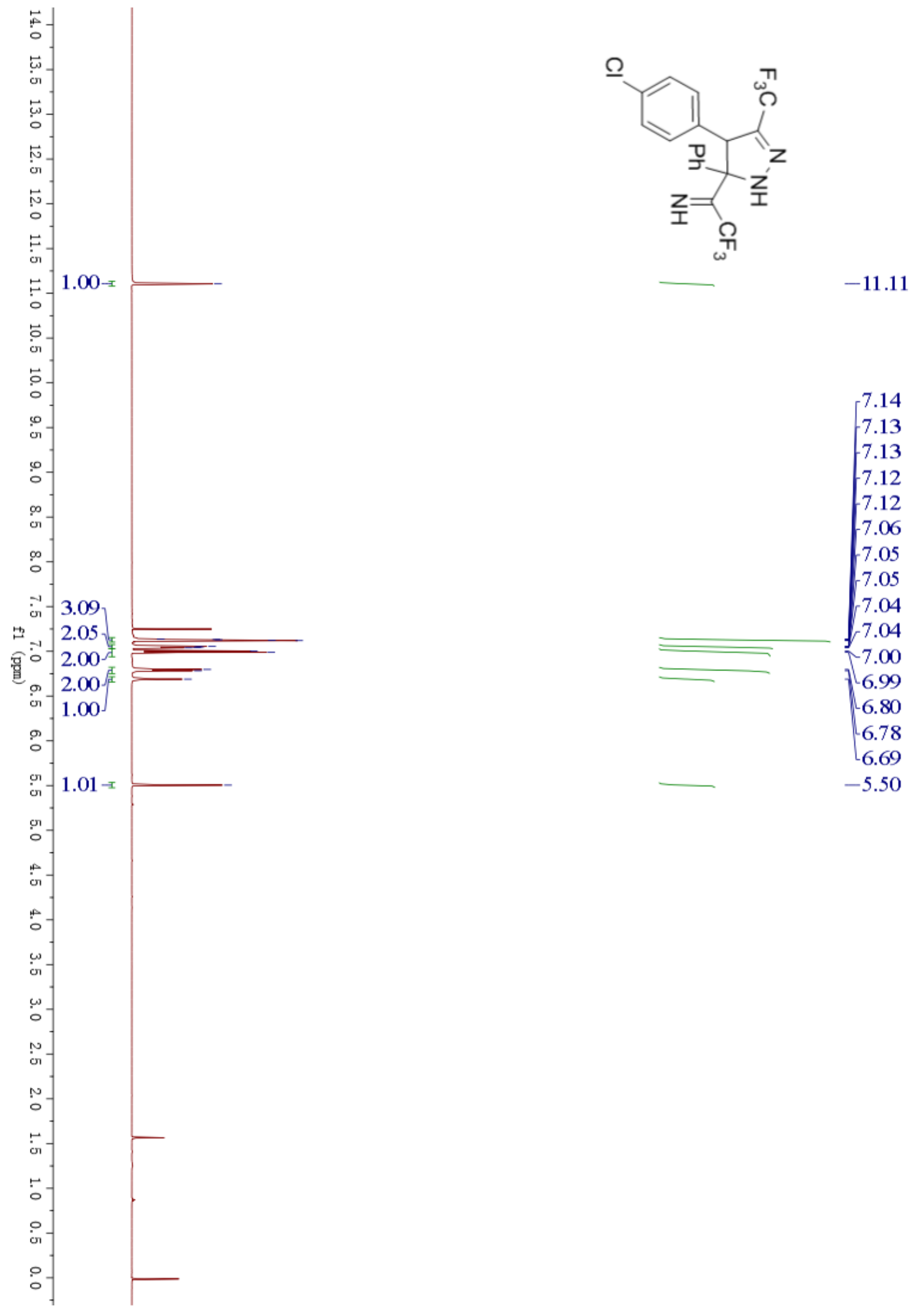
${ }^{13} \mathrm{C} \mathrm{NMR}\left(151 \mathrm{MHz}, \mathrm{CDCl}_{3}\right)$ spectrum of $\mathbf{2 q}$
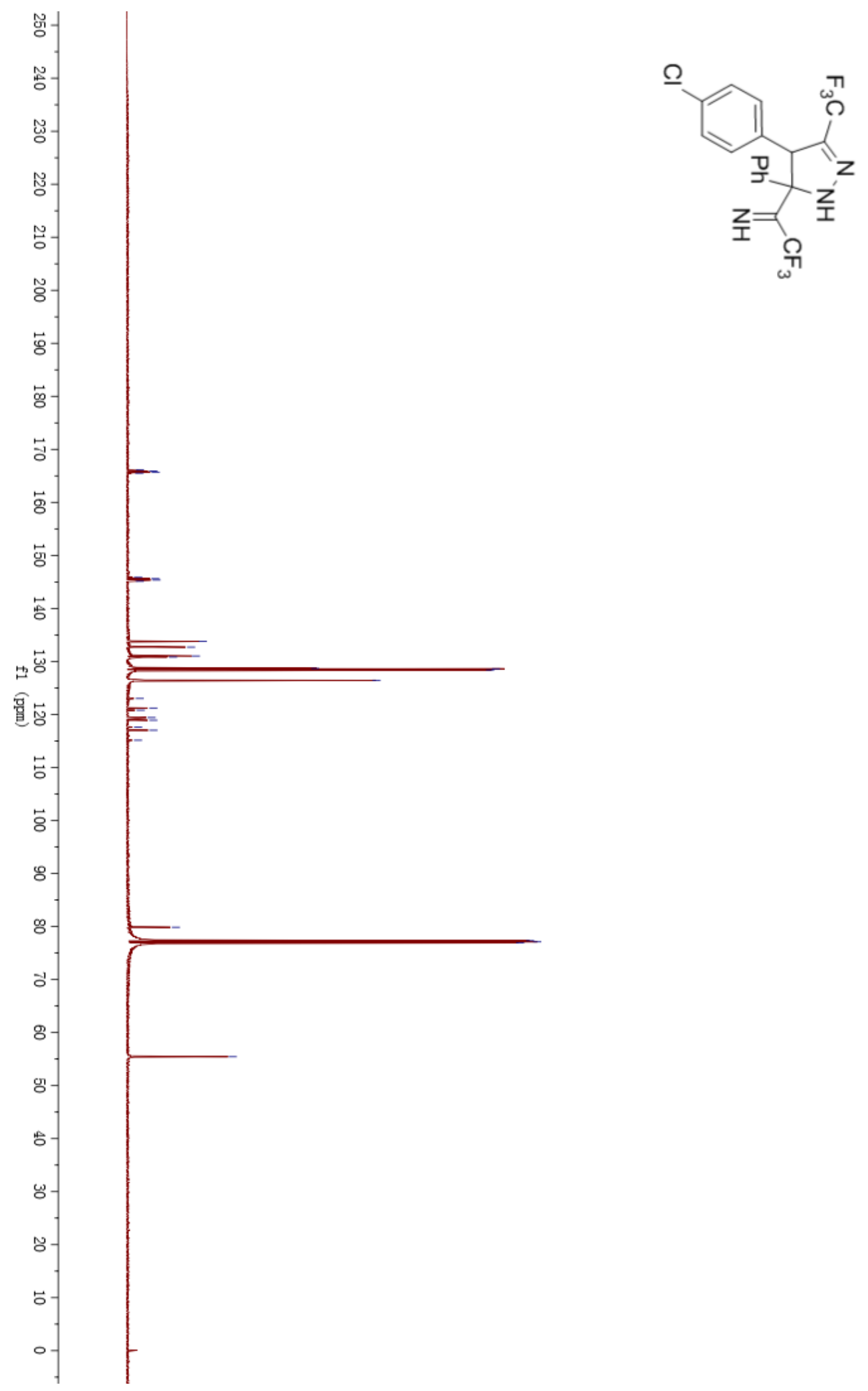

$\left\{\begin{array}{l}166.09 \\ 165.90 \\ 165.71 \\ 165.52\end{array}\right.$

165.52

145.85

145.61

$-145.36$

145.12

133.77

132.70

131.02

130.83

128.66

$-128.61$

128.39

$-126.45$

$-123.01$

$-121.21$

$-120.80$

119.42

118.91

117.62

$-117.02$

115.13

79.86

77.33

77.12

76.90

$-55.44$ 
${ }^{19} \mathrm{~F} \mathrm{NMR}\left(565 \mathrm{MHz}, \mathrm{CDCl}_{3}\right)$ spectrum of $\mathbf{2 q}$
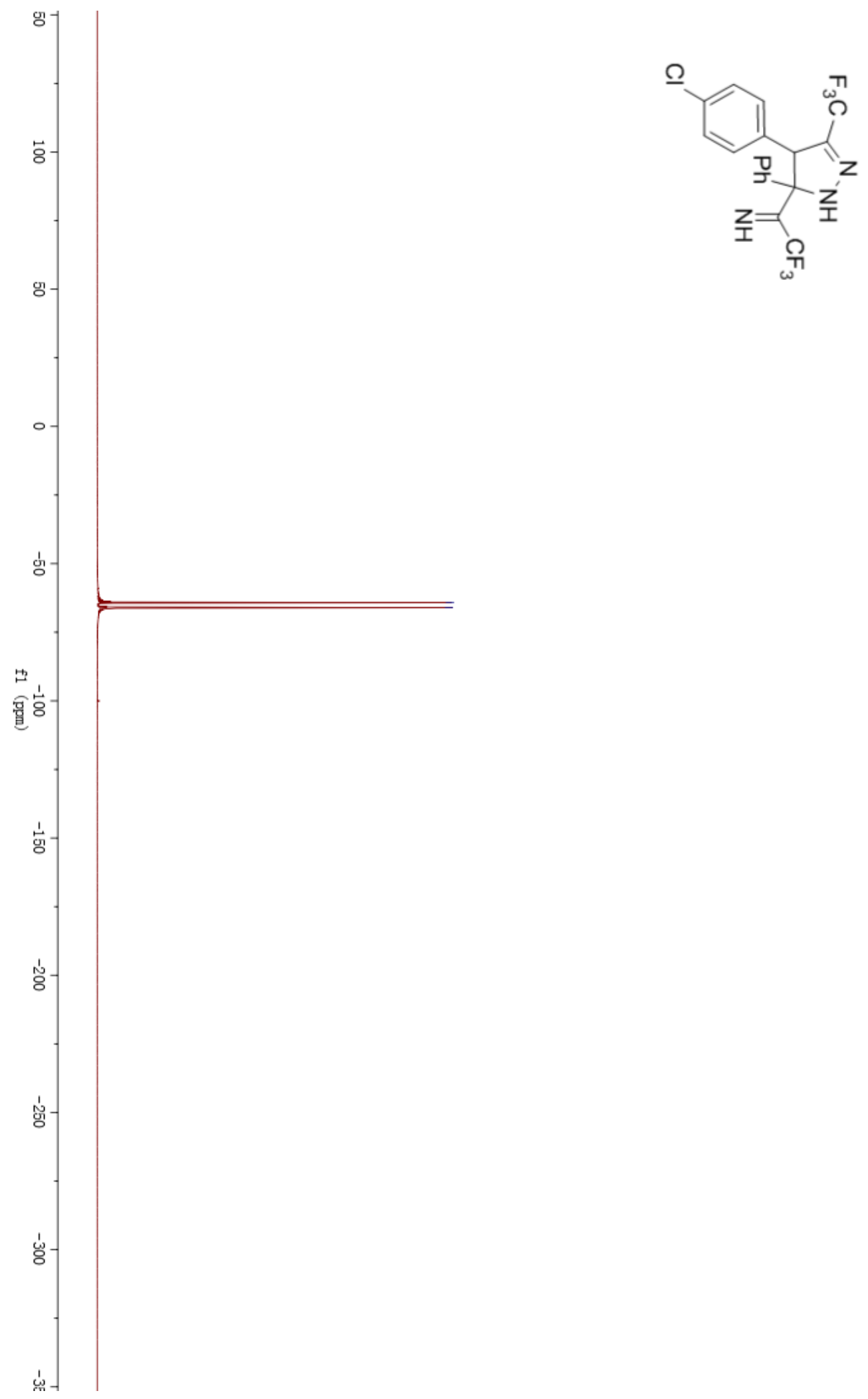

$-64.22$

-66.06 
${ }^{1} \mathrm{H}$ NMR (600 MHz, $\left.\mathrm{CDCl}_{3}\right)$ spectrum of $\mathbf{2 r}$
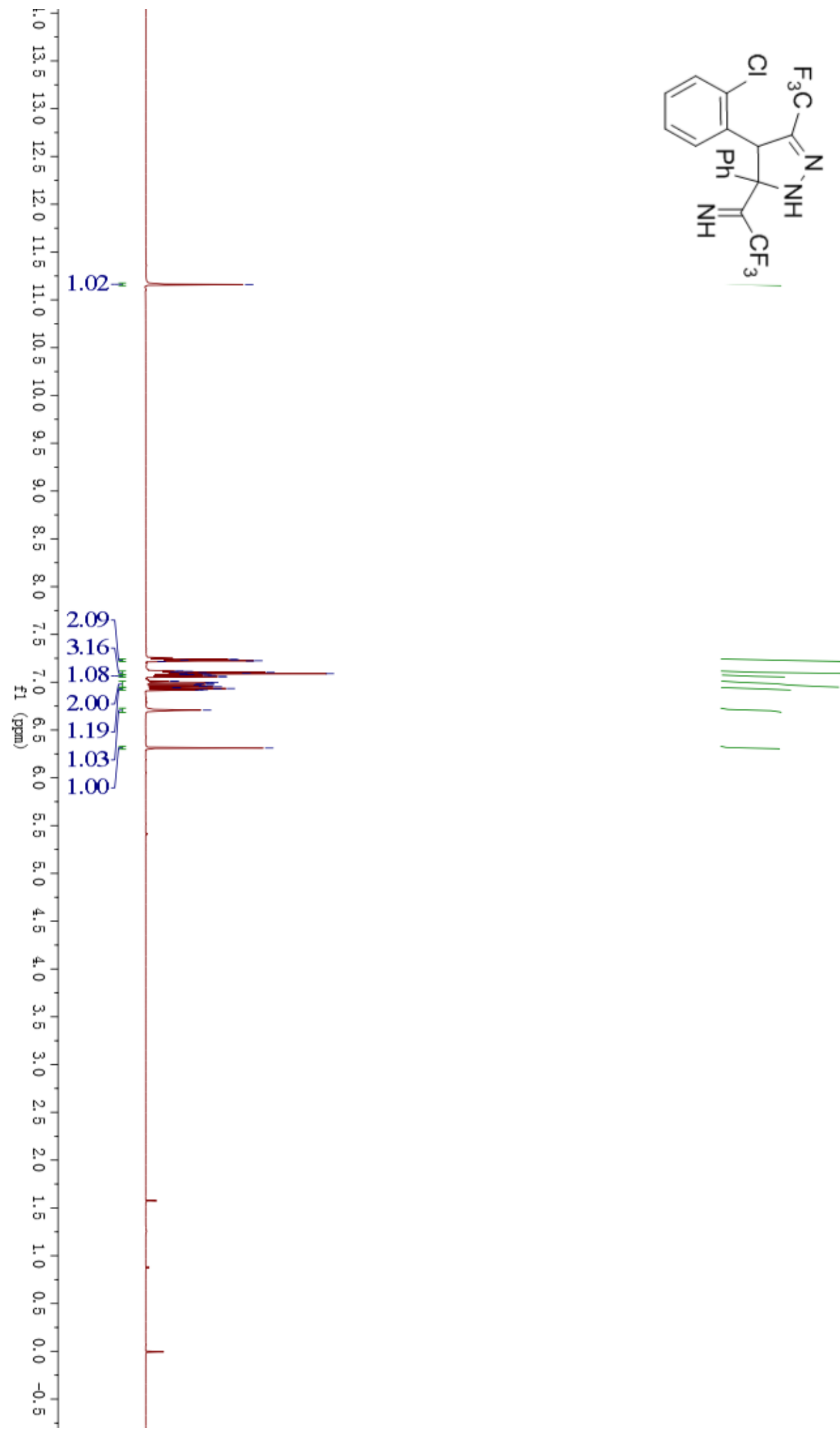

$-11.16$

7.24

$-7.23$

7.23

7.22

$-7.22$

7.22

7.12

7.11

7.11

7.10

7.10

7.10

7.09

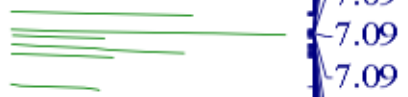

$\left[\begin{array}{l}7.09 \\ 7.08\end{array}\right.$

7.08

7.07

$-7.07$

$-7.06$

7.06

7.01

7.01

7.00

7.00

6.99

6.98

6.97

6.97

6.96

6.95

6.94

6.94

6.93

6.93

6.92

$-6.92$

6.71

6.31 
${ }^{13} \mathrm{C} \mathrm{NMR}\left(151 \mathrm{MHz}, \mathrm{CDCl}_{3}\right)$ spectrum of $\mathbf{2 r}$

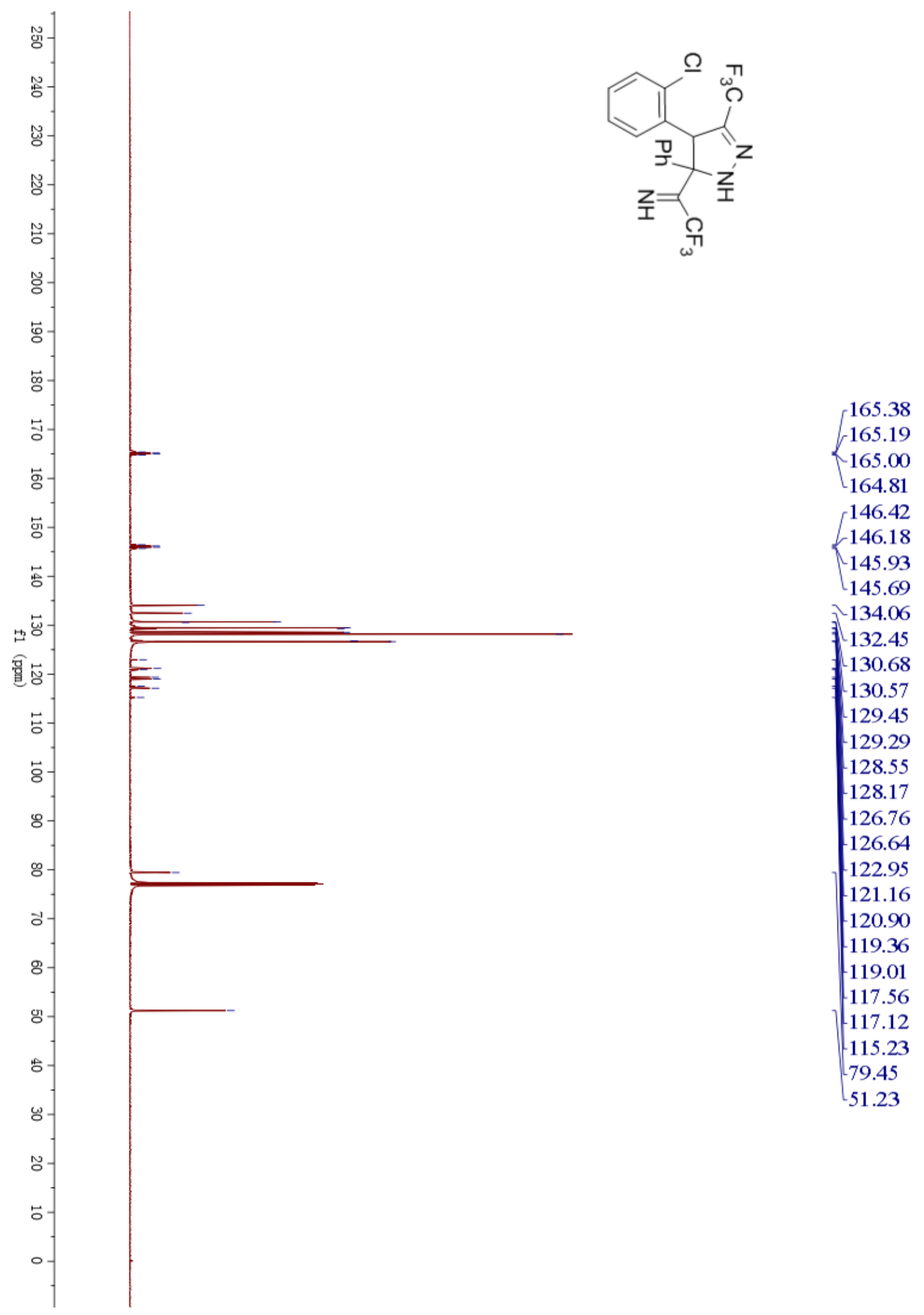


${ }^{19} \mathrm{~F}$ NMR $\left(565 \mathrm{MHz}, \mathrm{CDCl}_{3}\right)$ spectrum of $\mathbf{2 r}$

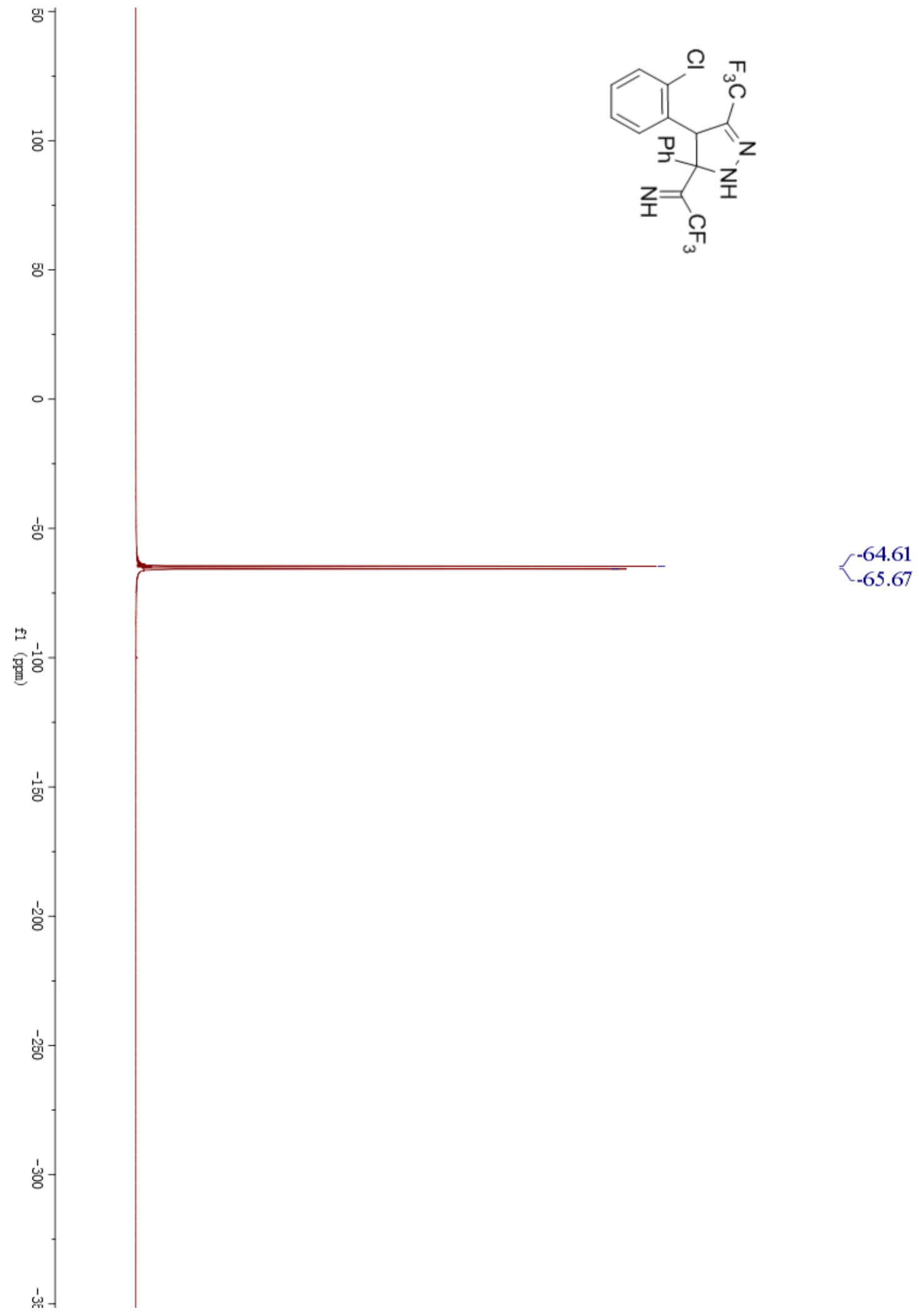


${ }^{1} \mathrm{H}$ NMR (600 MHz, $\mathrm{CDCl}_{3}$ ) spectrum of $\mathbf{2 s}$
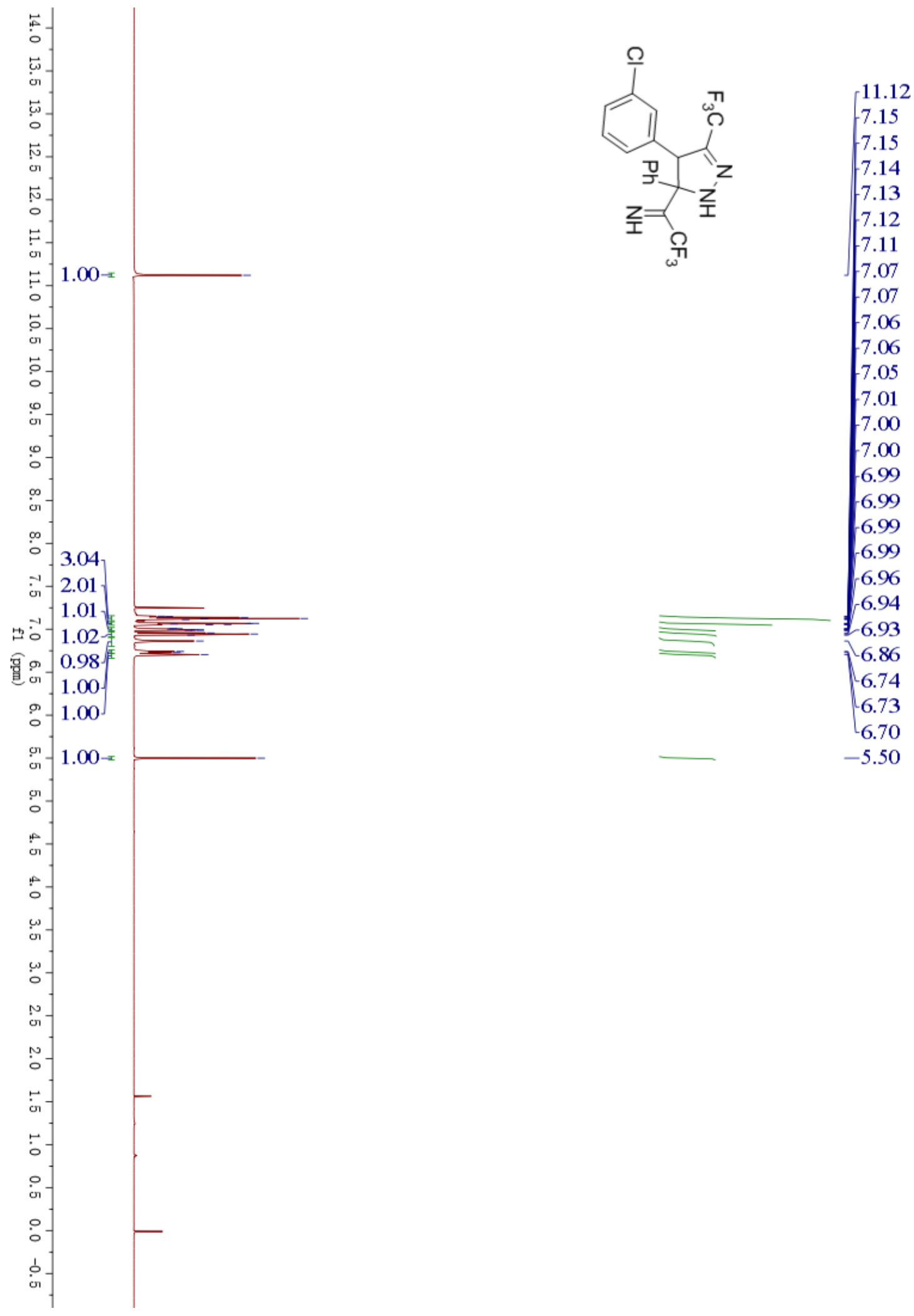
${ }^{13} \mathrm{C} \mathrm{NMR}\left(151 \mathrm{MHz}, \mathrm{CDCl}_{3}\right)$ spectrum of $2 \mathrm{~s}$

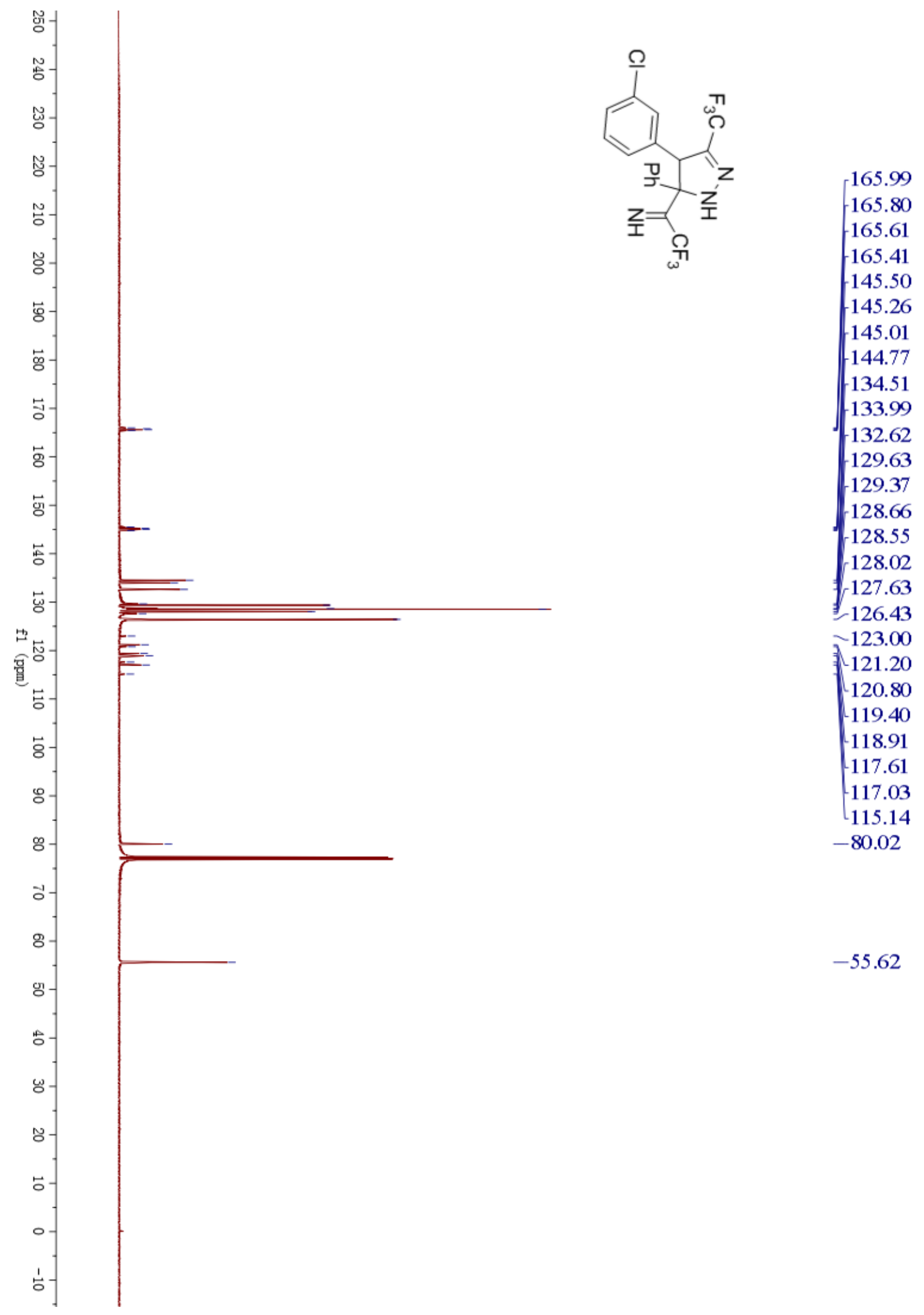


${ }^{19} \mathrm{~F}$ NMR $\left(565 \mathrm{MHz}, \mathrm{CDCl}_{3}\right)$ spectrum of $2 \mathrm{~s}$

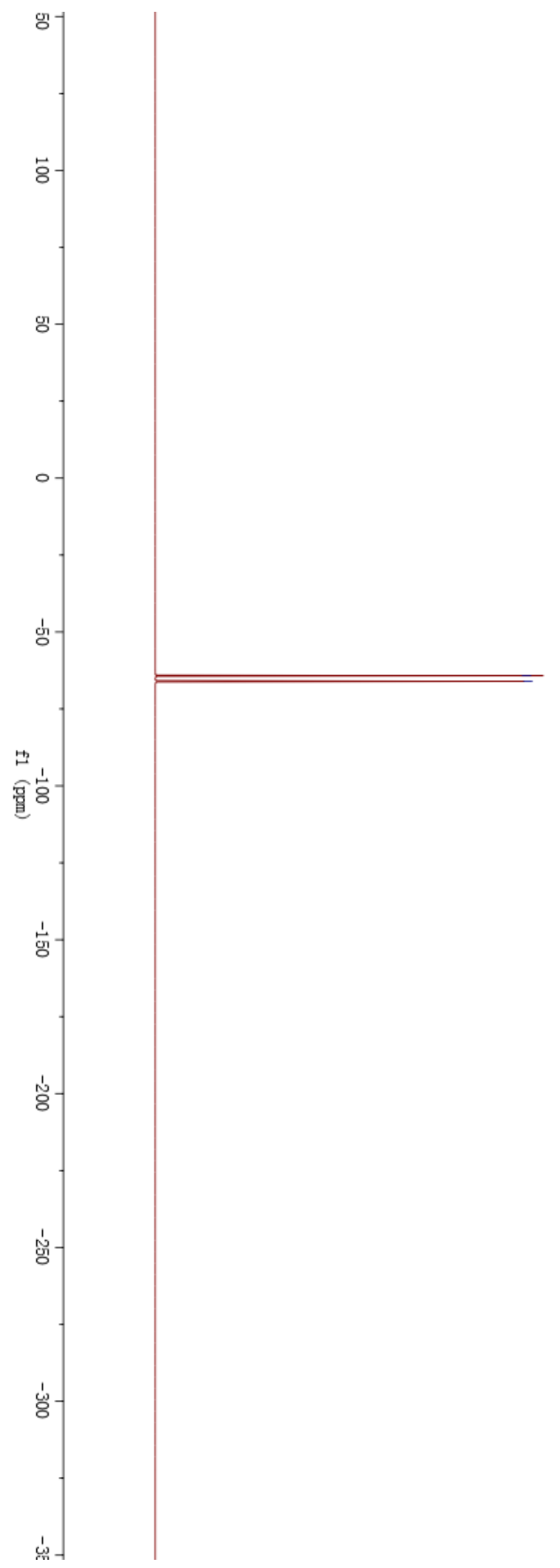


${ }^{1} \mathrm{H}$ NMR (600 MHz, $\mathrm{CDCl}_{3}$ ) spectrum of $\mathbf{2 t}$
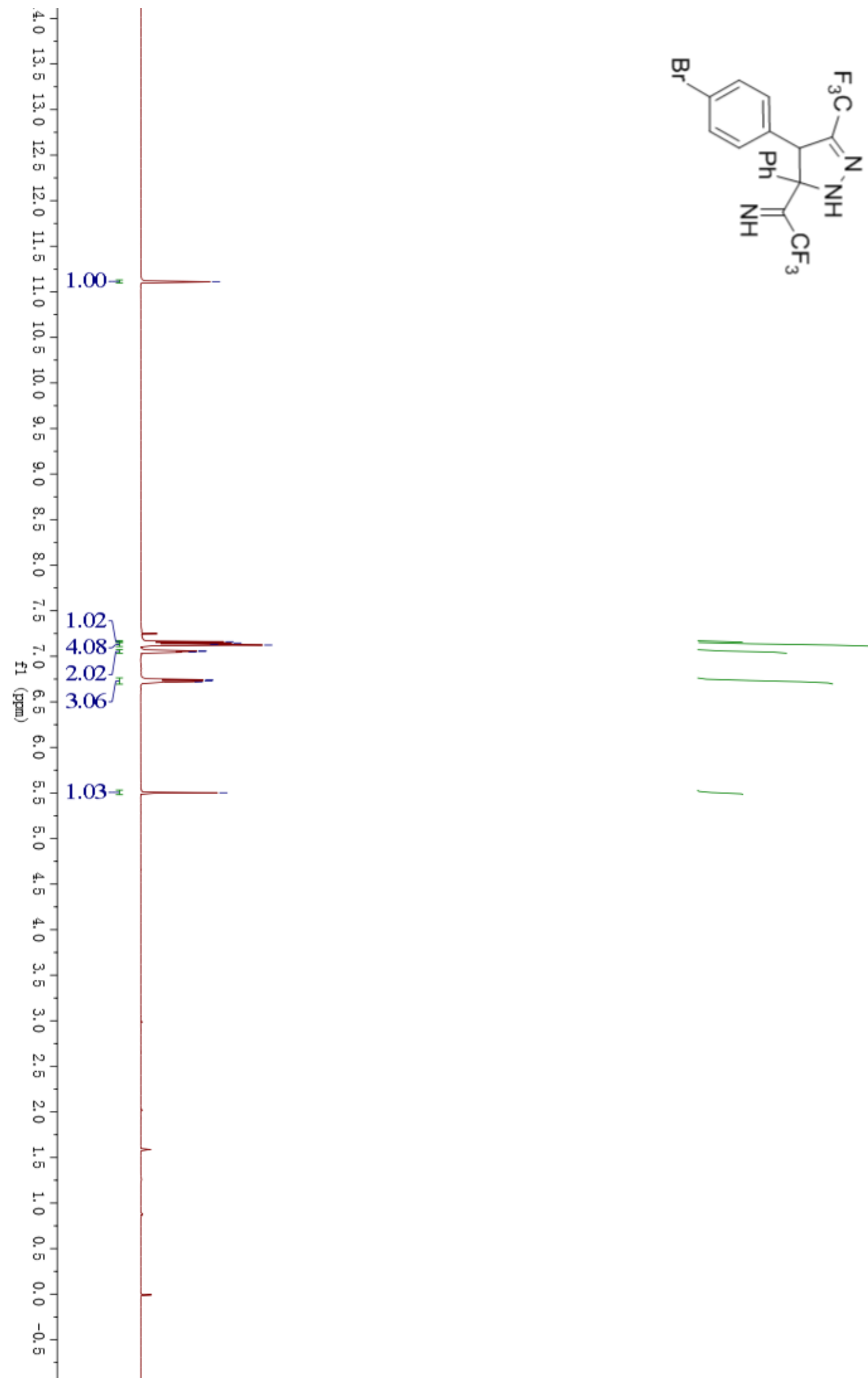

$-11.11$

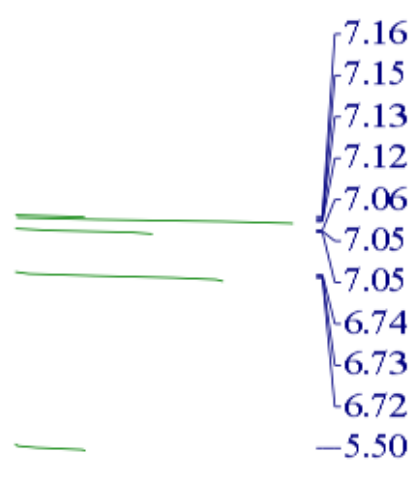


${ }^{13} \mathrm{C}$ NMR (151 MHz, $\left.\mathrm{CDCl}_{3}\right)$ spectrum of $\mathbf{2 t}$
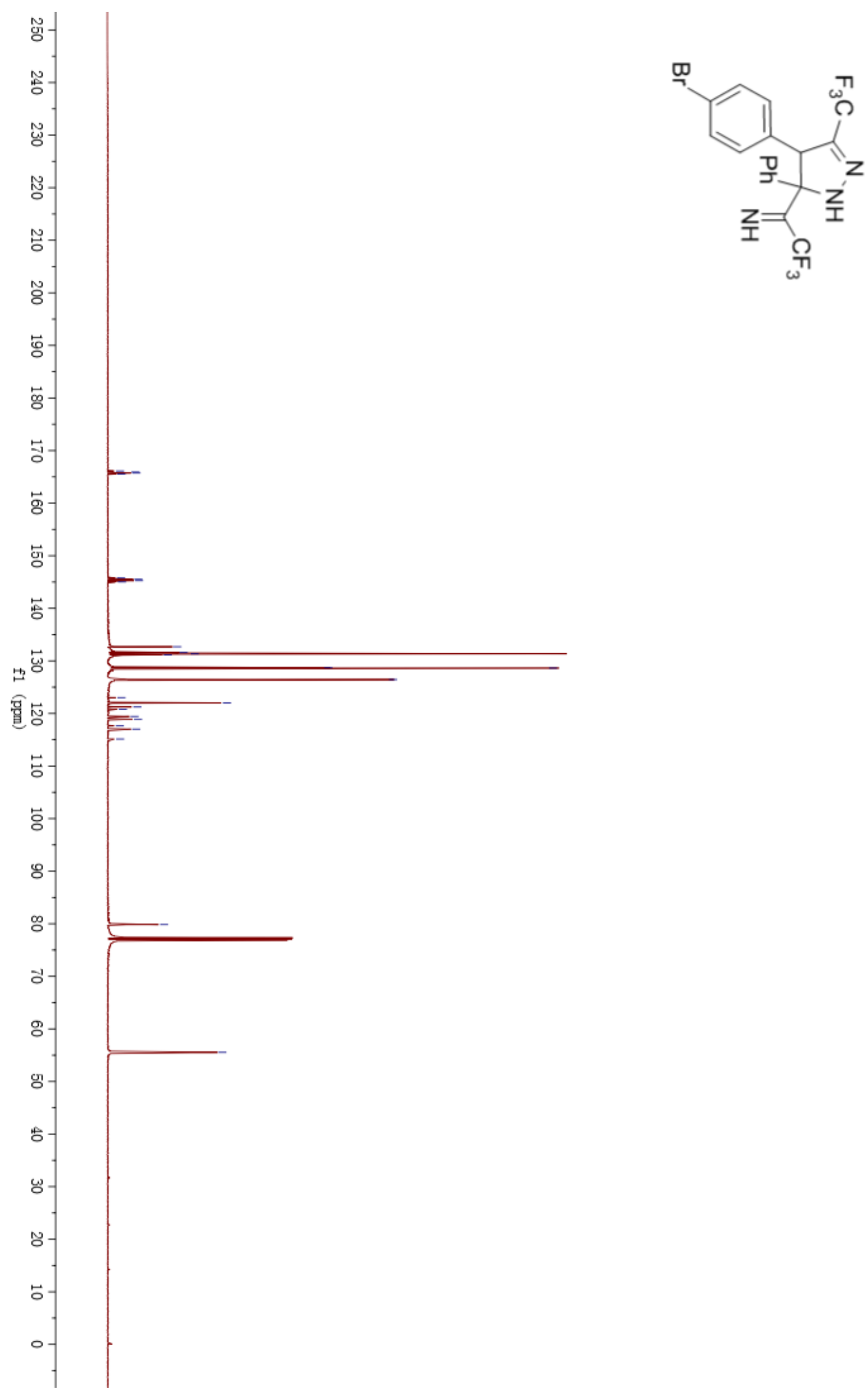

166.11

165.92

165.72

165.53

145.75

145.51

145.26

145.02

$-132.69$

131.57

131.33

$-131.16$

$-128.68$

128.63

$-126.45$

123.02

$-122.01$

121.22

$-120.80$

119.43

118.91

117.63

$-117.02$

$-115.13$

79.85

55.54 
${ }^{19} \mathrm{~F}$ NMR (565 MHz, $\mathrm{CDCl}_{3}$ ) spectrum of $\mathbf{2 t}$
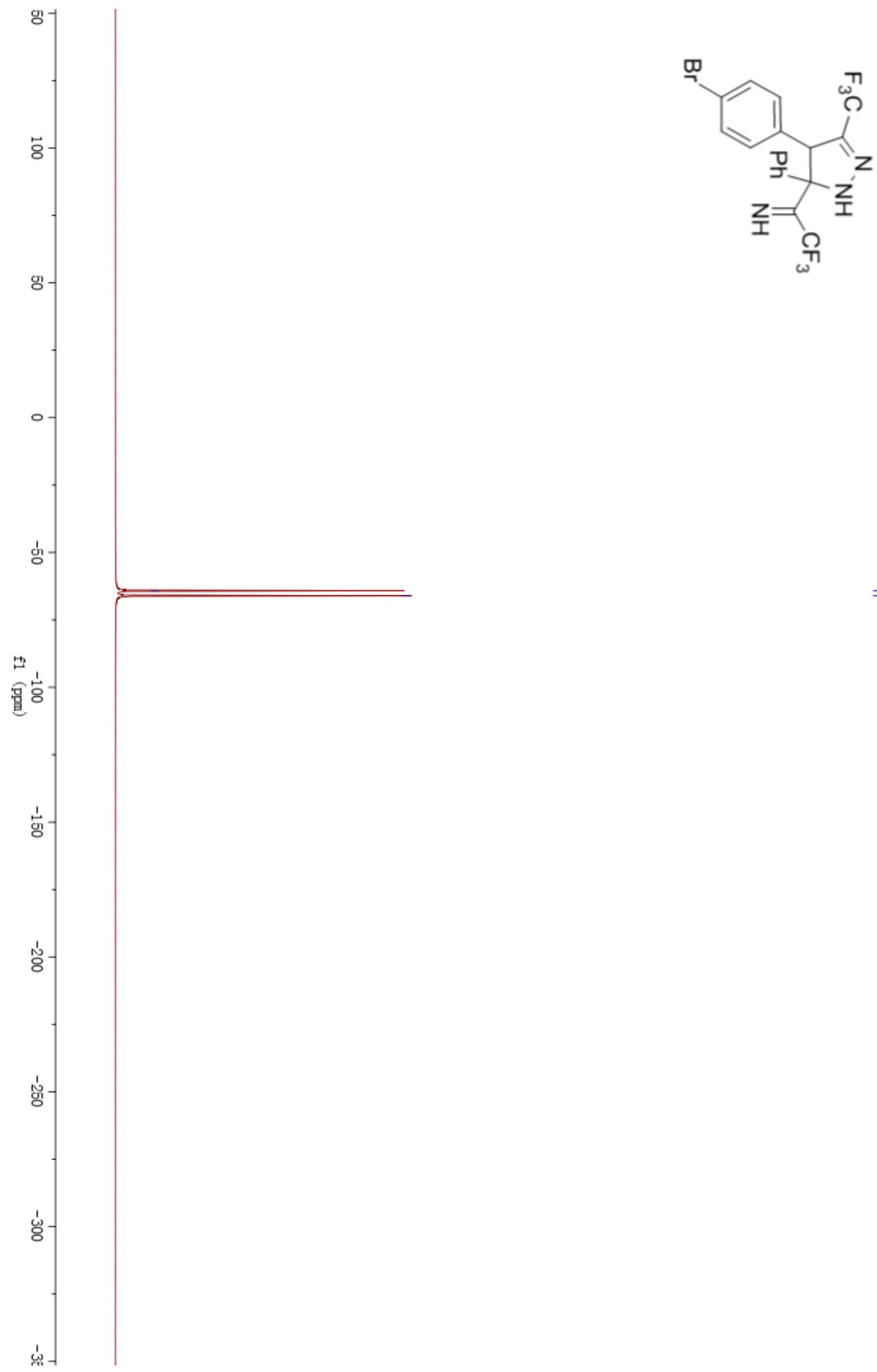

$-64.25$

$-66.08$ 
${ }^{1} \mathrm{H}$ NMR $\left(600 \mathrm{MHz}, \mathrm{CDCl}_{3}\right)$ spectrum of $\mathbf{2} \mathbf{u}$
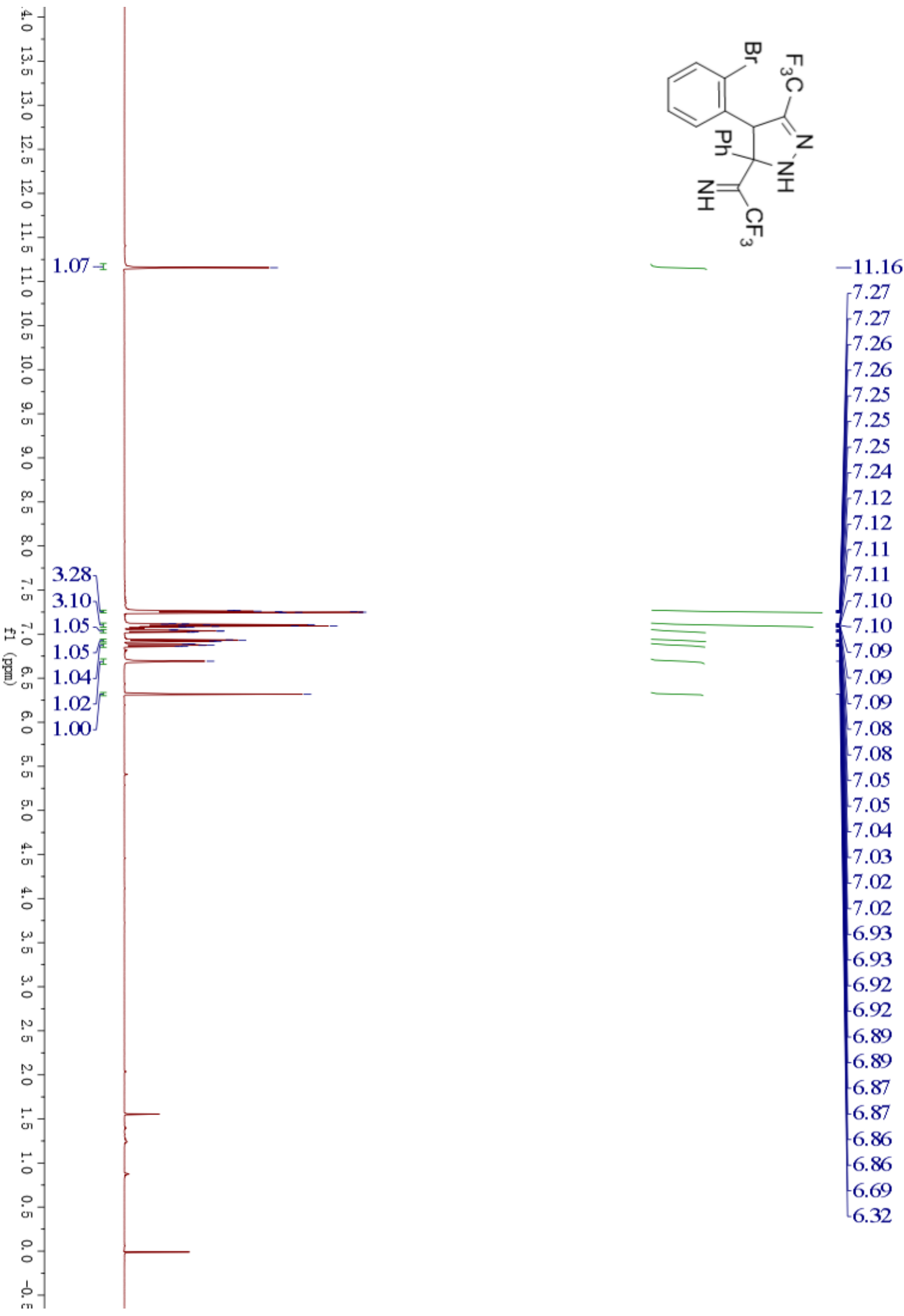
${ }^{13} \mathrm{C} \mathrm{NMR}\left(151 \mathrm{MHz}, \mathrm{CDCl}_{3}\right)$ spectrum of $\mathbf{2 u}$

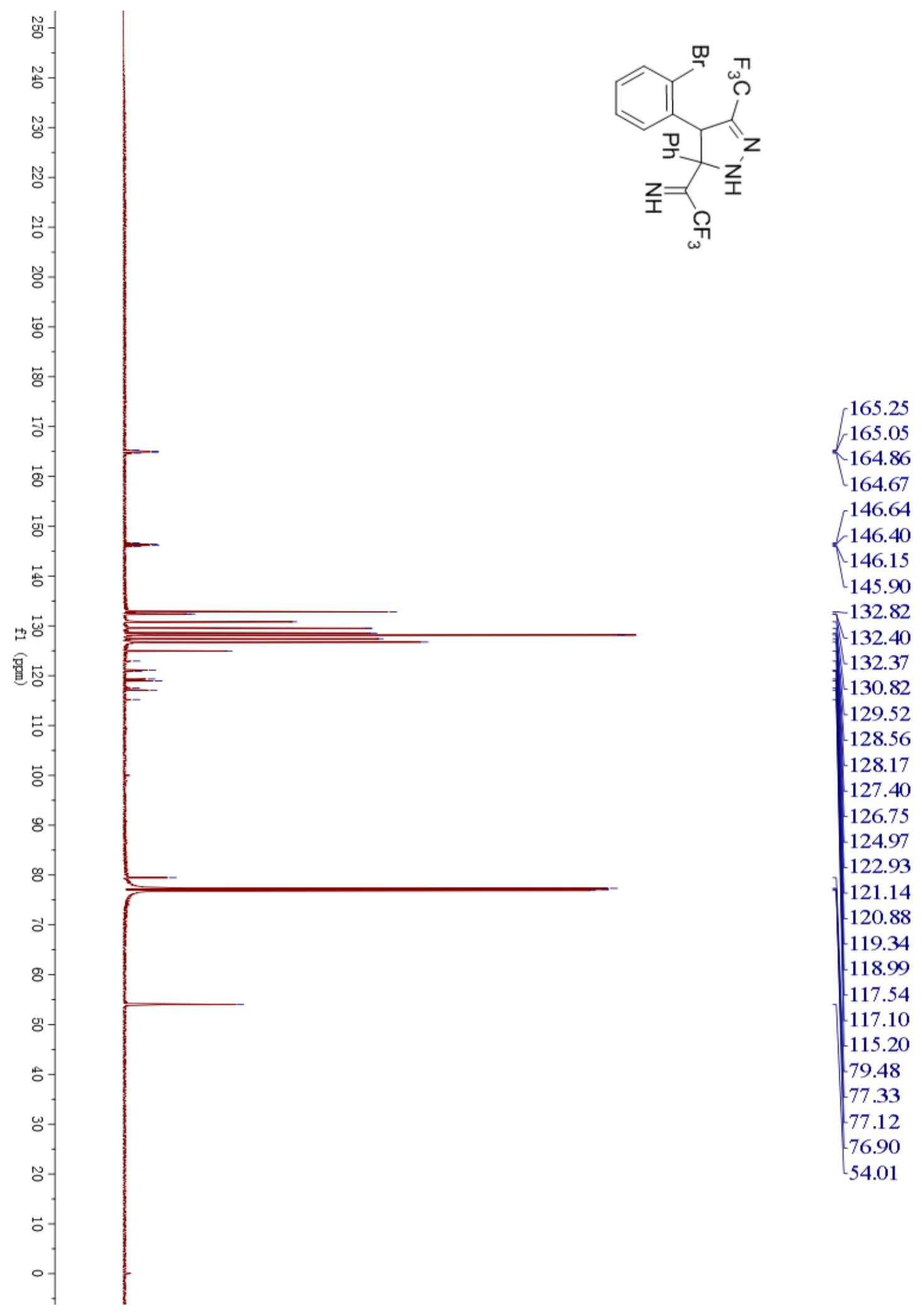


${ }^{19} \mathrm{~F} \mathrm{NMR}\left(565 \mathrm{MHz}, \mathrm{CDCl}_{3}\right)$ spectrum of $\mathbf{2 u}$

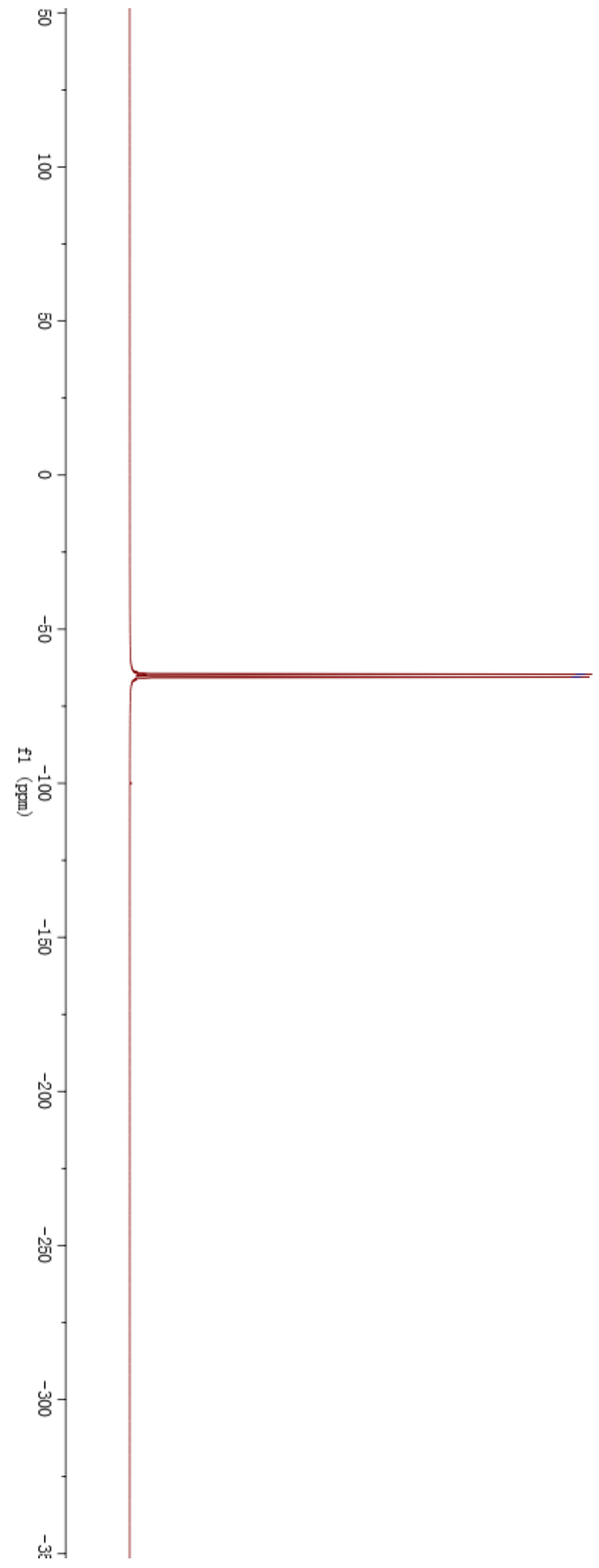


${ }^{1} \mathrm{H}$ NMR (600 MHz, $\mathrm{CDCl}_{3}$ ) spectrum of $\mathbf{2 v}$

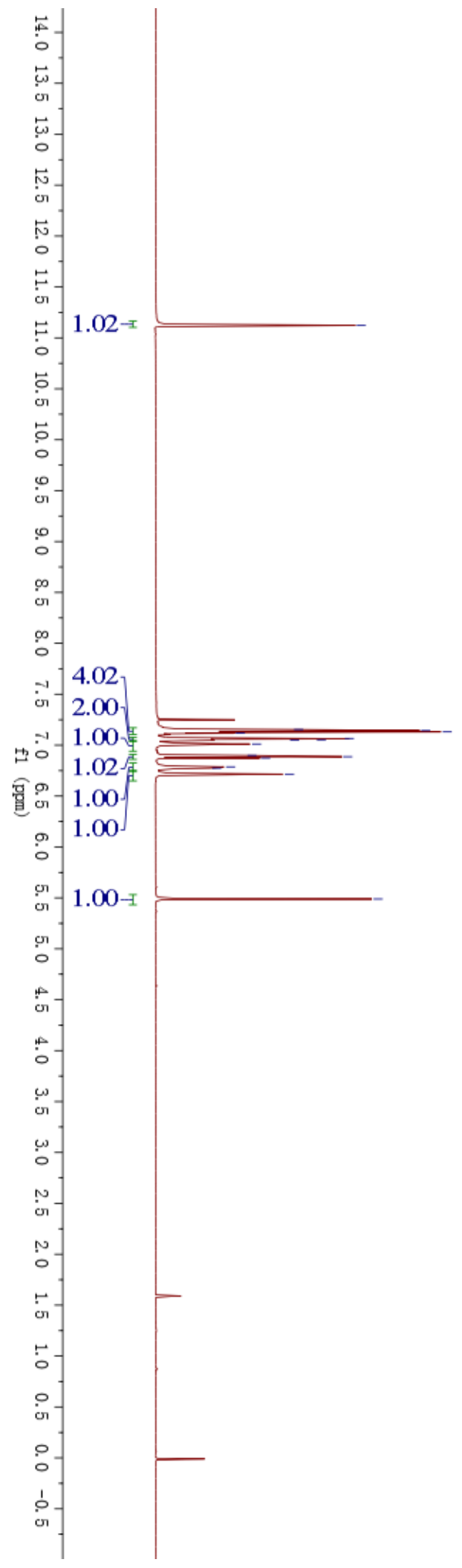

$-11.12$

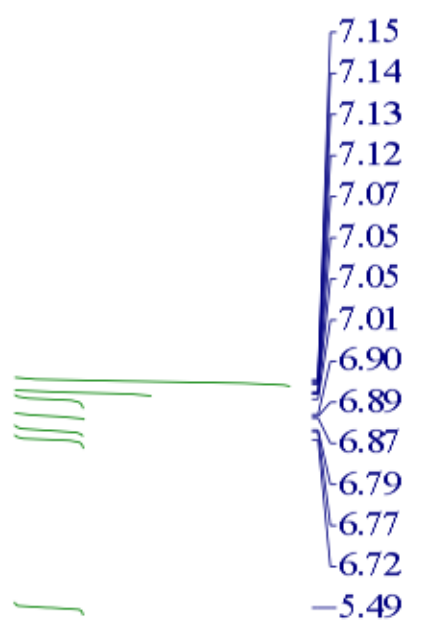


${ }^{13} \mathrm{C}$ NMR $\left(151 \mathrm{MHz}, \mathrm{CDCl}_{3}\right)$ spectrum of $\mathbf{2 v}$
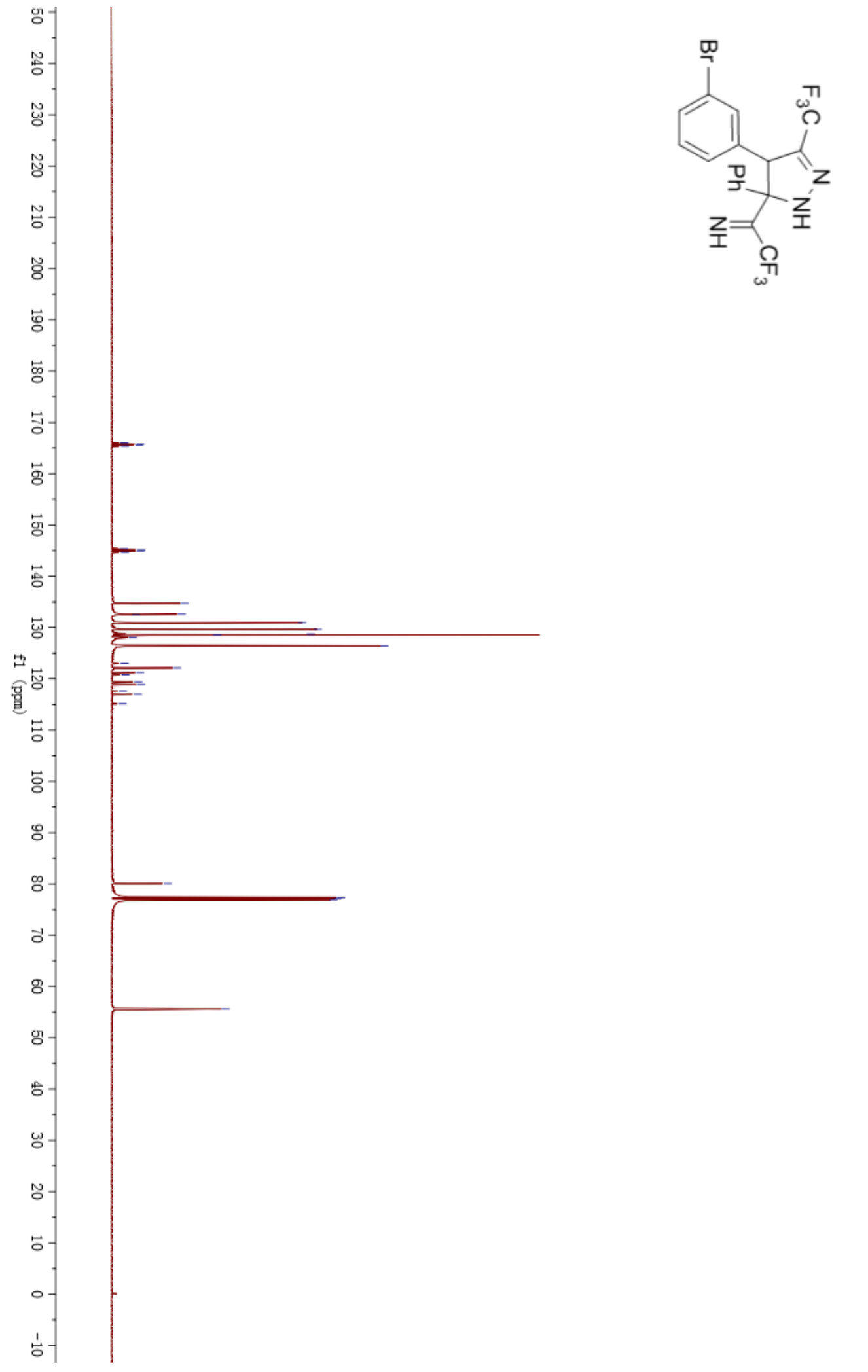

165.97

165.78

165.59

$-165.39$

145.42
145.18

144.93

144.69

134.76

132.61

132.54

130.93

129.65

128.69

128.54

128.04

126.42

123.00

122.13

$-121.20$

120.80

119.41

118.91

$-117.61$

$-117.02$

115.13

80.06

77.34

77.12

76.91

$-55.60$ 
$\left.{ }^{19} \mathrm{~F} \mathrm{NMR} \mathrm{(565} \mathrm{MHz,} \mathrm{CDCl}_{3}\right)$ spectrum of $\mathbf{2 v}$

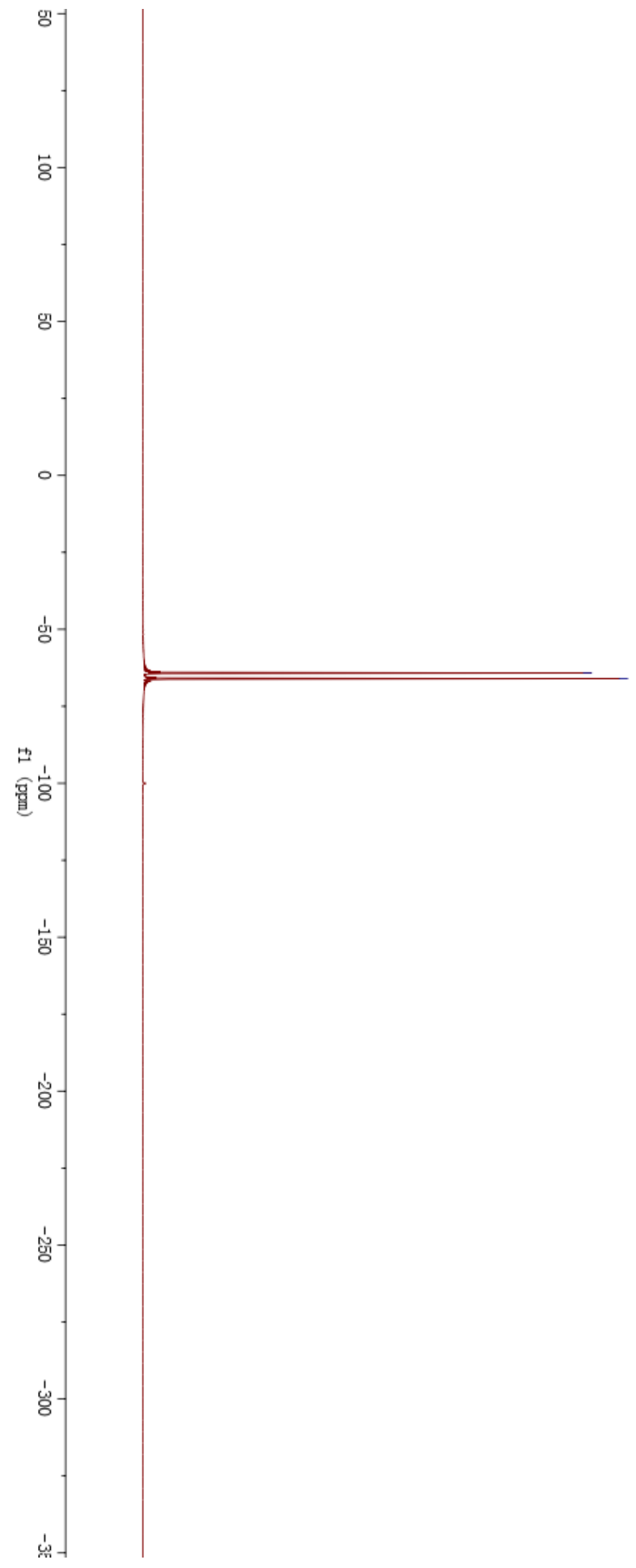

$-64.20$

$\checkmark 66.04$ 
${ }^{1} \mathrm{H}$ NMR $\left(600 \mathrm{MHz}, \mathrm{CDCl}_{3}\right)$ spectrum of $\mathbf{2 w}$

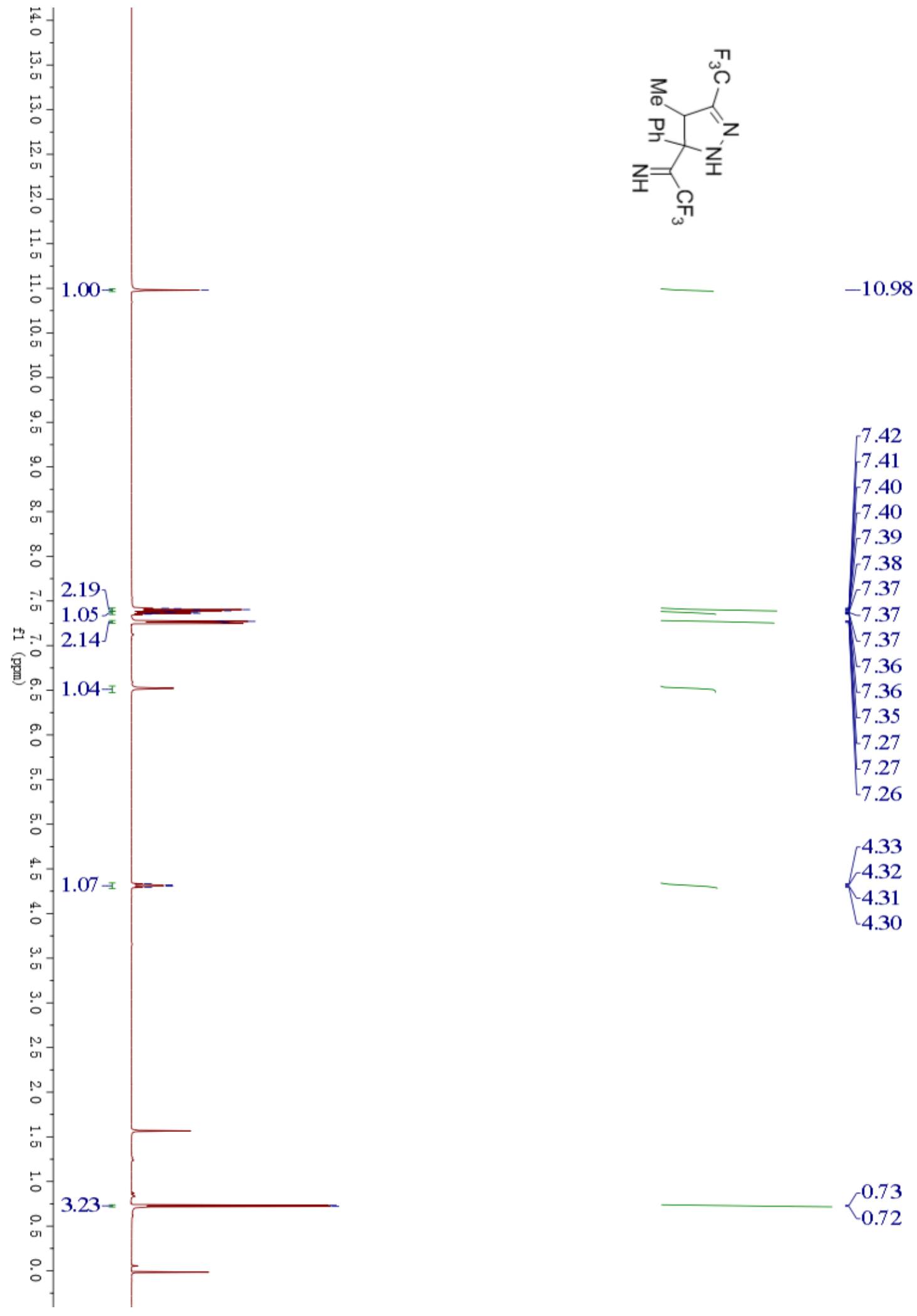


${ }^{13} \mathrm{C} \mathrm{NMR}\left(151 \mathrm{MHz}, \mathrm{CDCl}_{3}\right)$ spectrum of $\mathbf{2 w}$
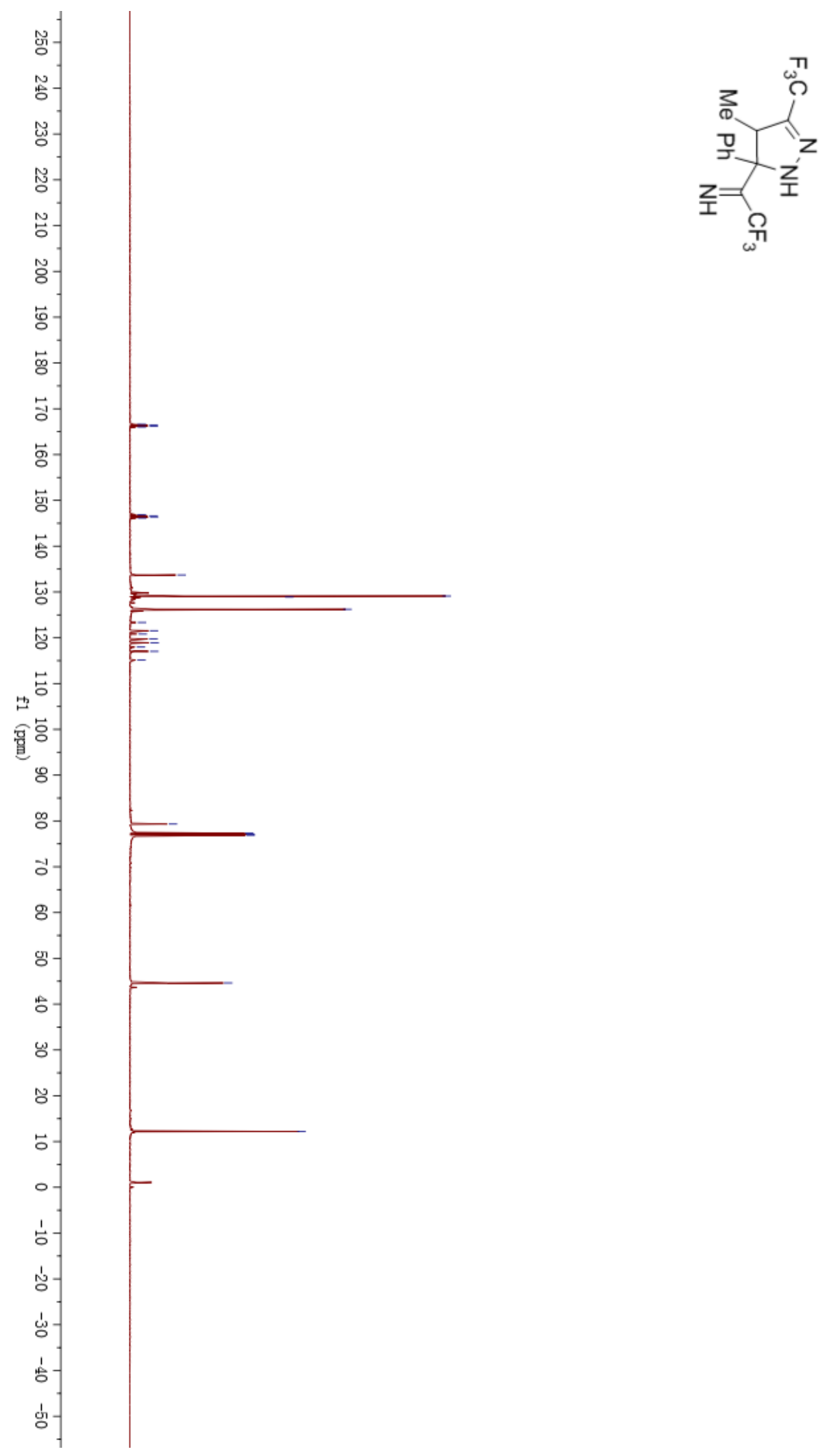

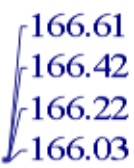

146.86

146.62

146.38

146.14

133.71

129.11

128.89

126.21

123.34

$-121.55$

$-120.81$

$-119.75$

$-118.92$

117.96

$-117.03$

115.14

79.32

$-77.32$

$-77.10$

76.89

44.63

$-12.19$ 
$\left.{ }^{19} \mathrm{~F} \mathrm{NMR} \mathrm{(565} \mathrm{MHz,} \mathrm{CDCl}_{3}\right)$ spectrum of $\mathbf{2 w}$

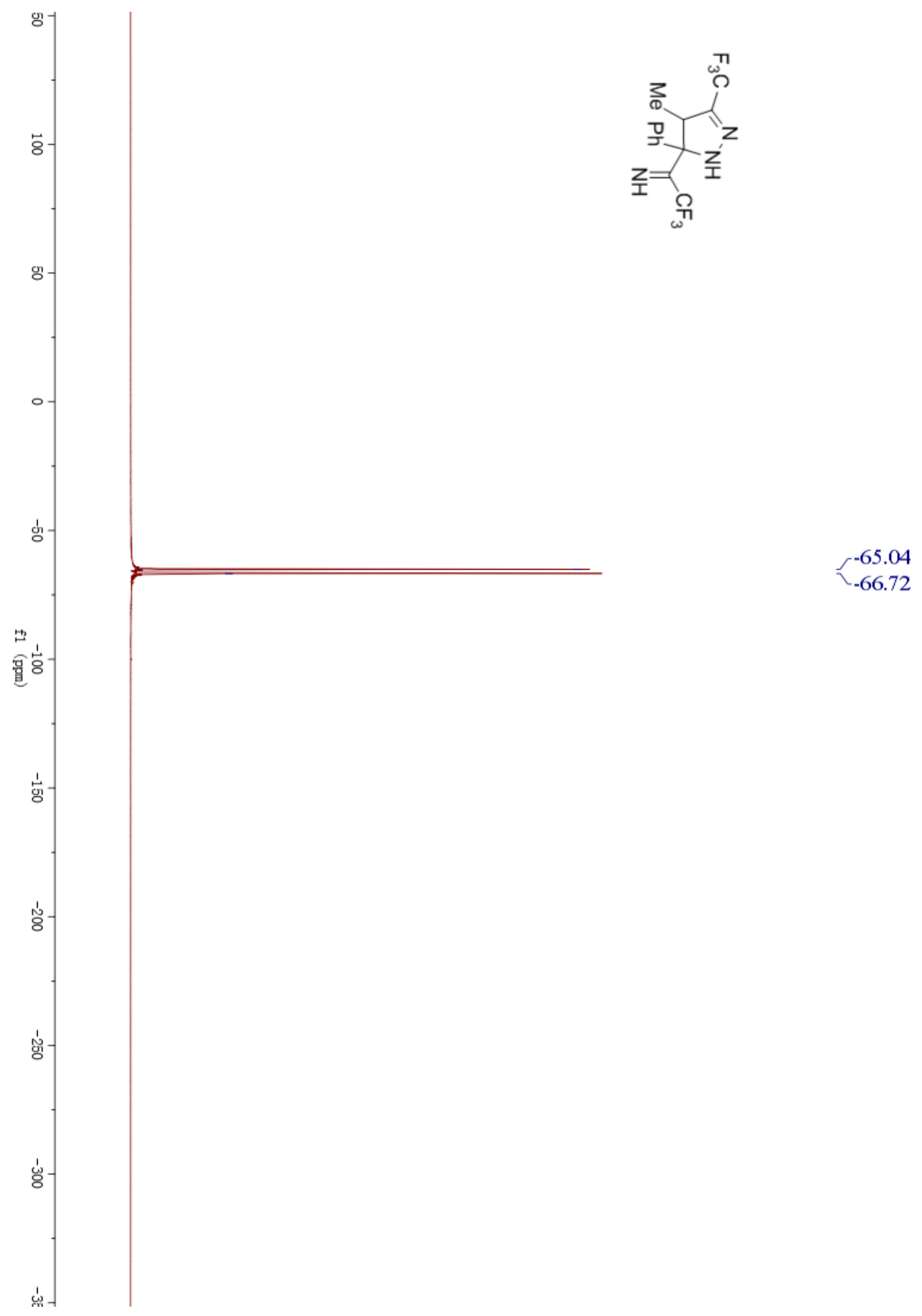


${ }^{1} \mathrm{H}$ NMR (600 MHz, $\mathrm{CDCl}_{3}$ ) spectrum of $\mathbf{2 x}$
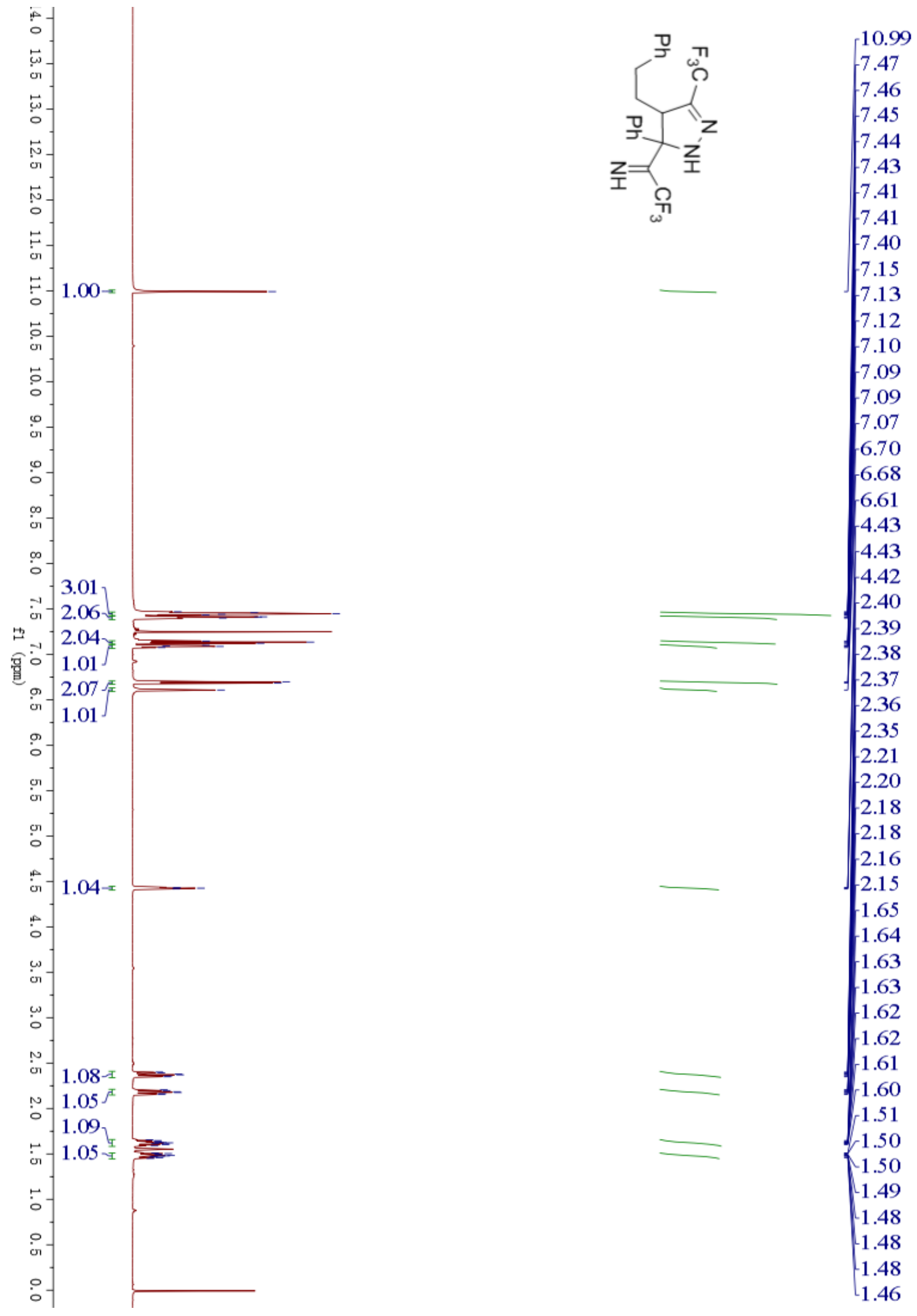
${ }^{13} \mathrm{C} \mathrm{NMR}\left(151 \mathrm{MHz}, \mathrm{CDCl}_{3}\right)$ spectrum of $\mathbf{2 x}$

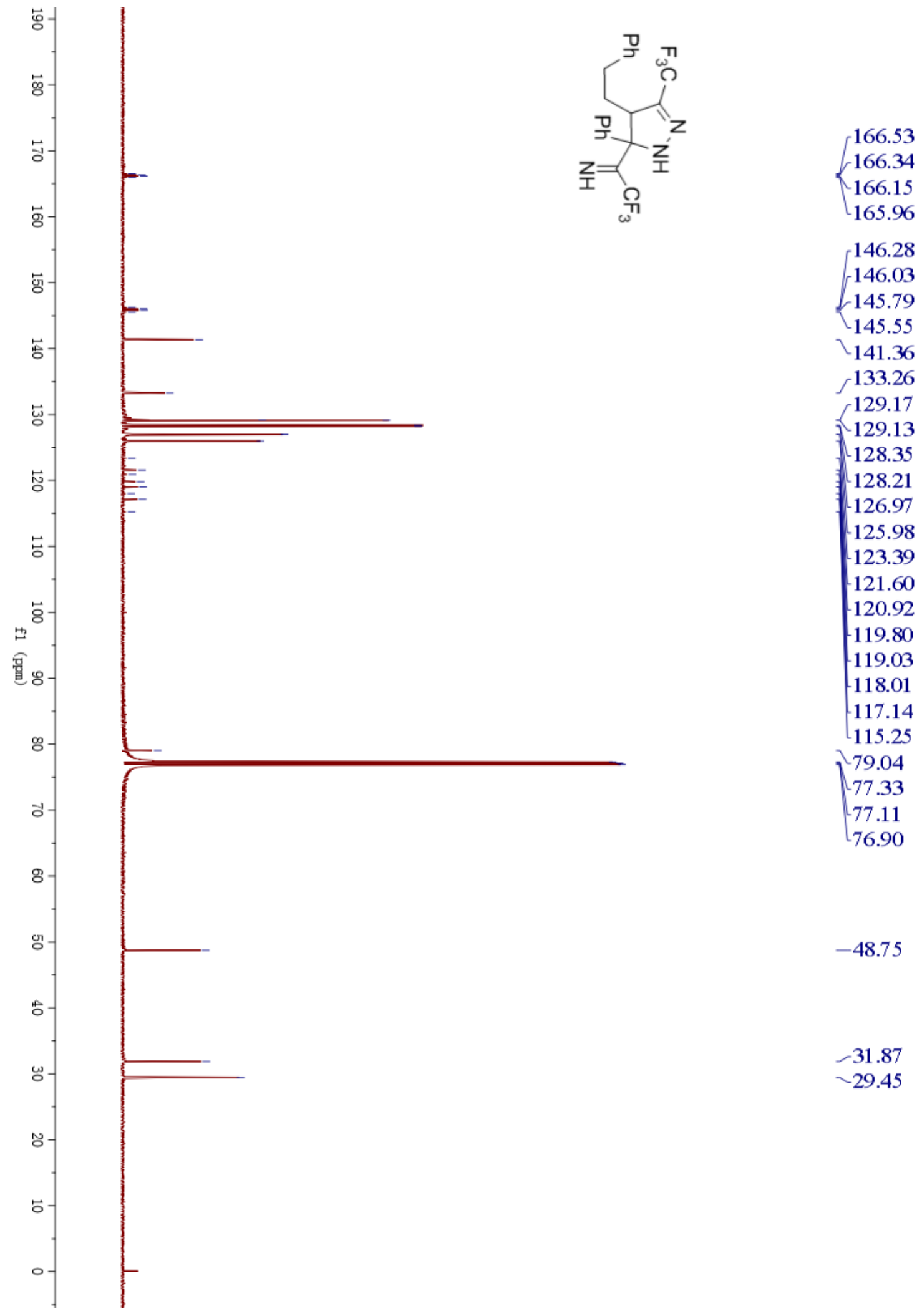


${ }^{19} \mathrm{~F}$ NMR $\left(565 \mathrm{MHz}, \mathrm{CDCl}_{3}\right)$ spectrum of $\mathbf{2 x}$

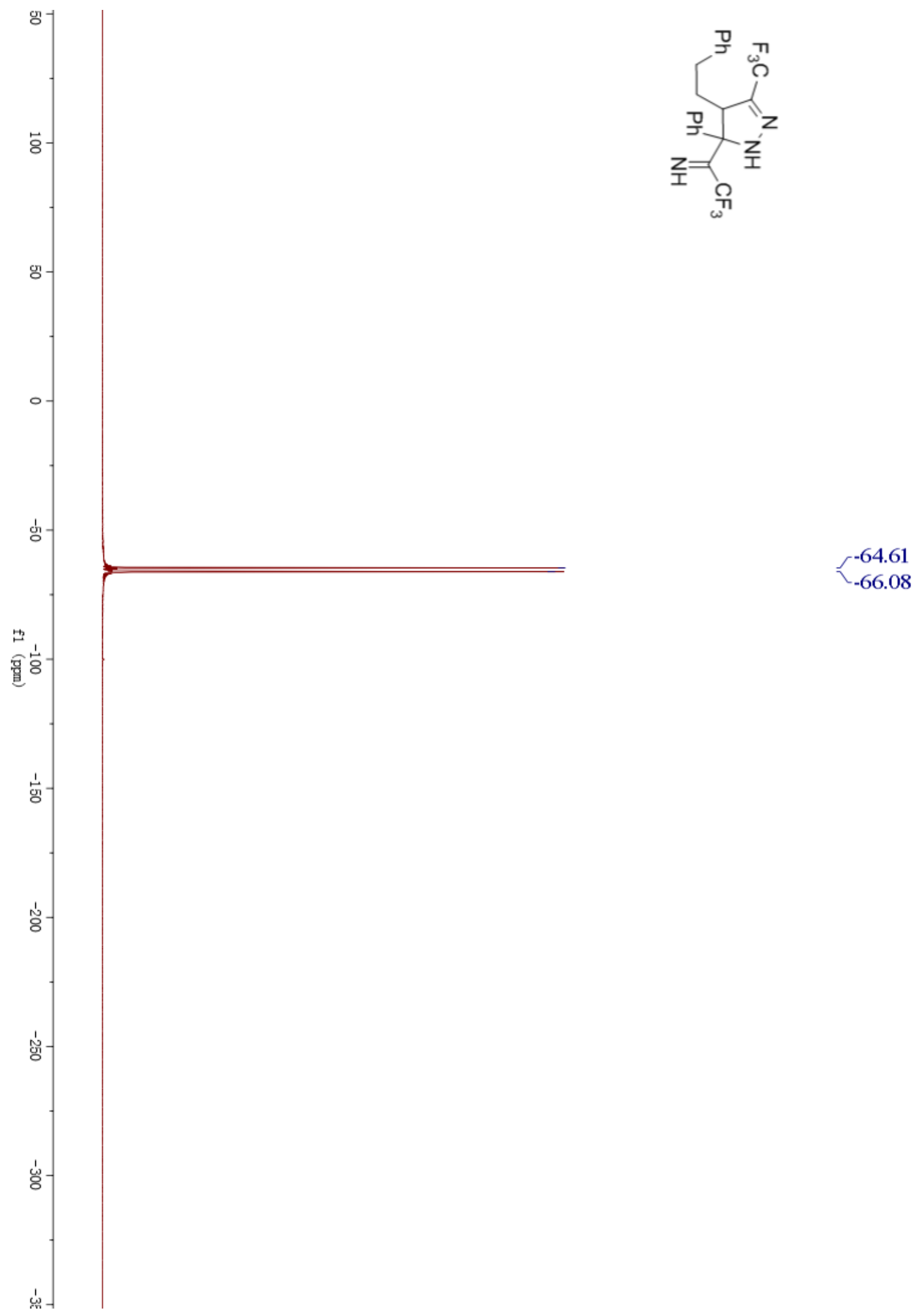


${ }^{1} \mathrm{H}$ NMR (600 MHz, $\left.\mathrm{CDCl}_{3}\right)$ spectrum of $\mathbf{5 a}$

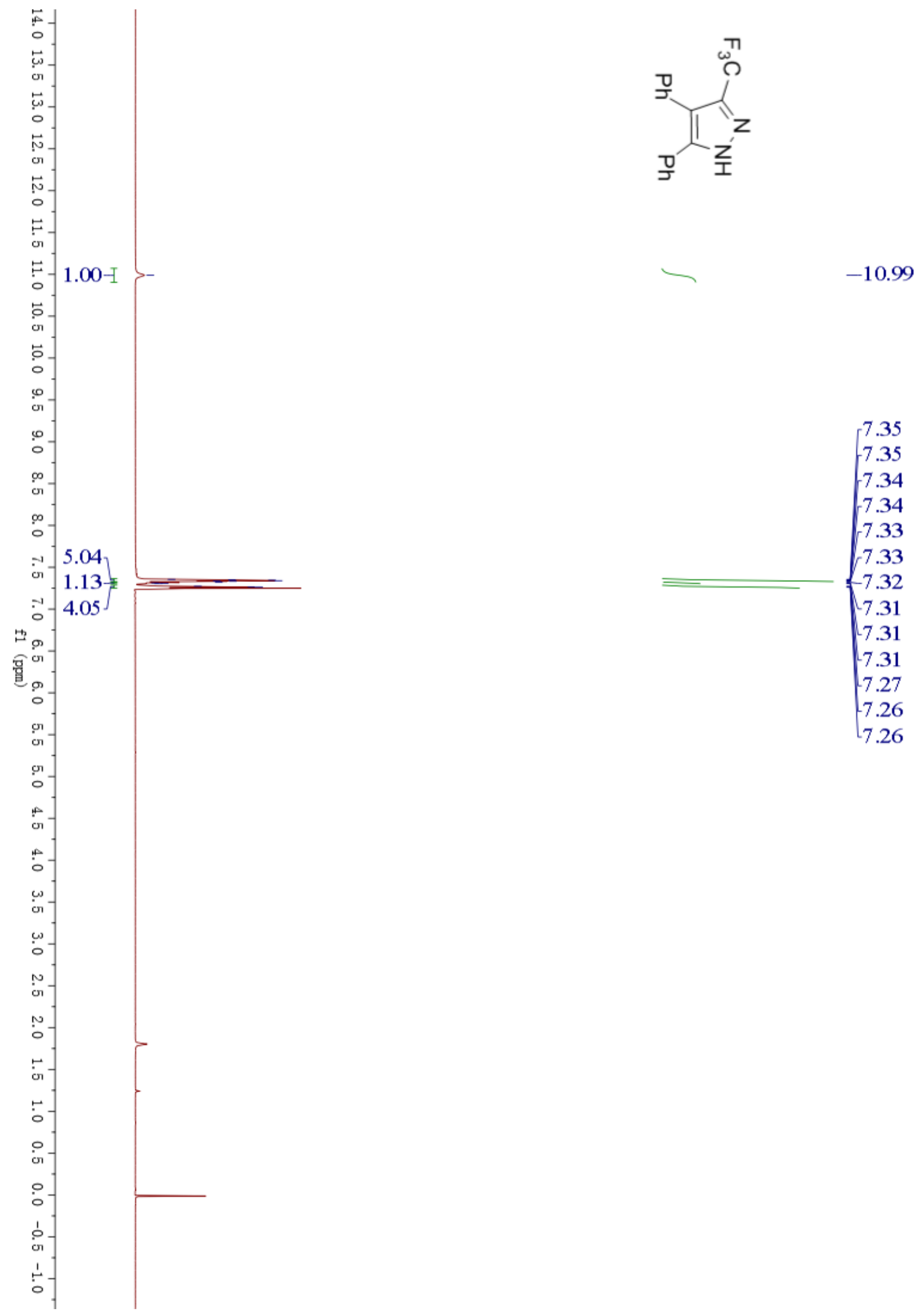


${ }^{13} \mathrm{C}$ NMR (151 MHz, DMSO-d6) spectrum of 5a
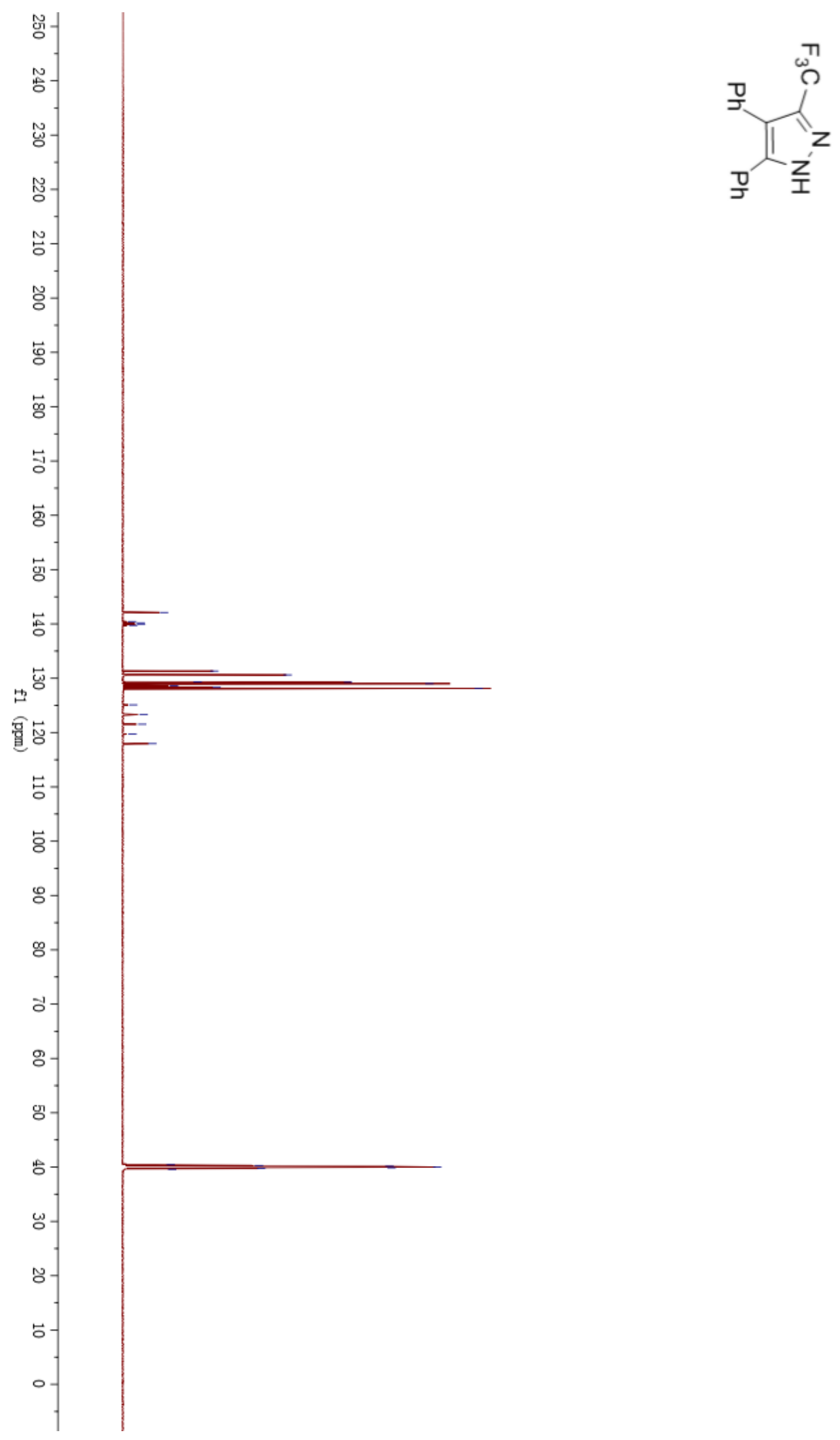

142.11

140.43

140.19

$-139.96$

139.73

131.32

130.62

129.32

129.25

129.00

128.61

128.32

$-128.15$

$-125.10$

123.32

121.53

$-119.75$

117.96

40.42

40.28

40.15

$-40.01$

39.87

39.73

39.59 
${ }^{19}$ F NMR (565 MHz, DMSO-d6) spectrum of 5a

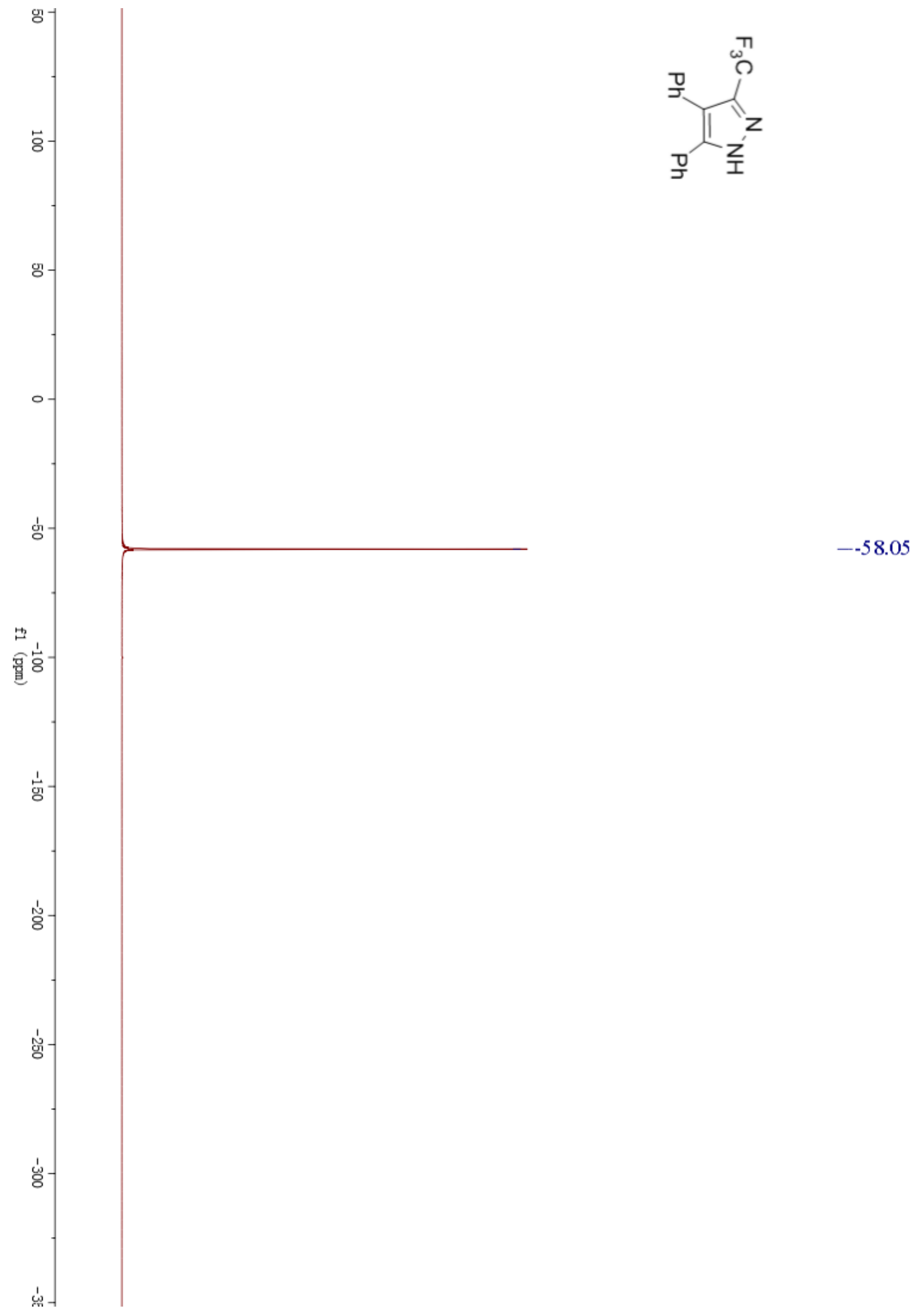


${ }^{1} \mathrm{H}$ NMR $\left(600 \mathrm{MHz}, \mathrm{CDCl}_{3}\right)$ spectrum of $\mathbf{5 b}$

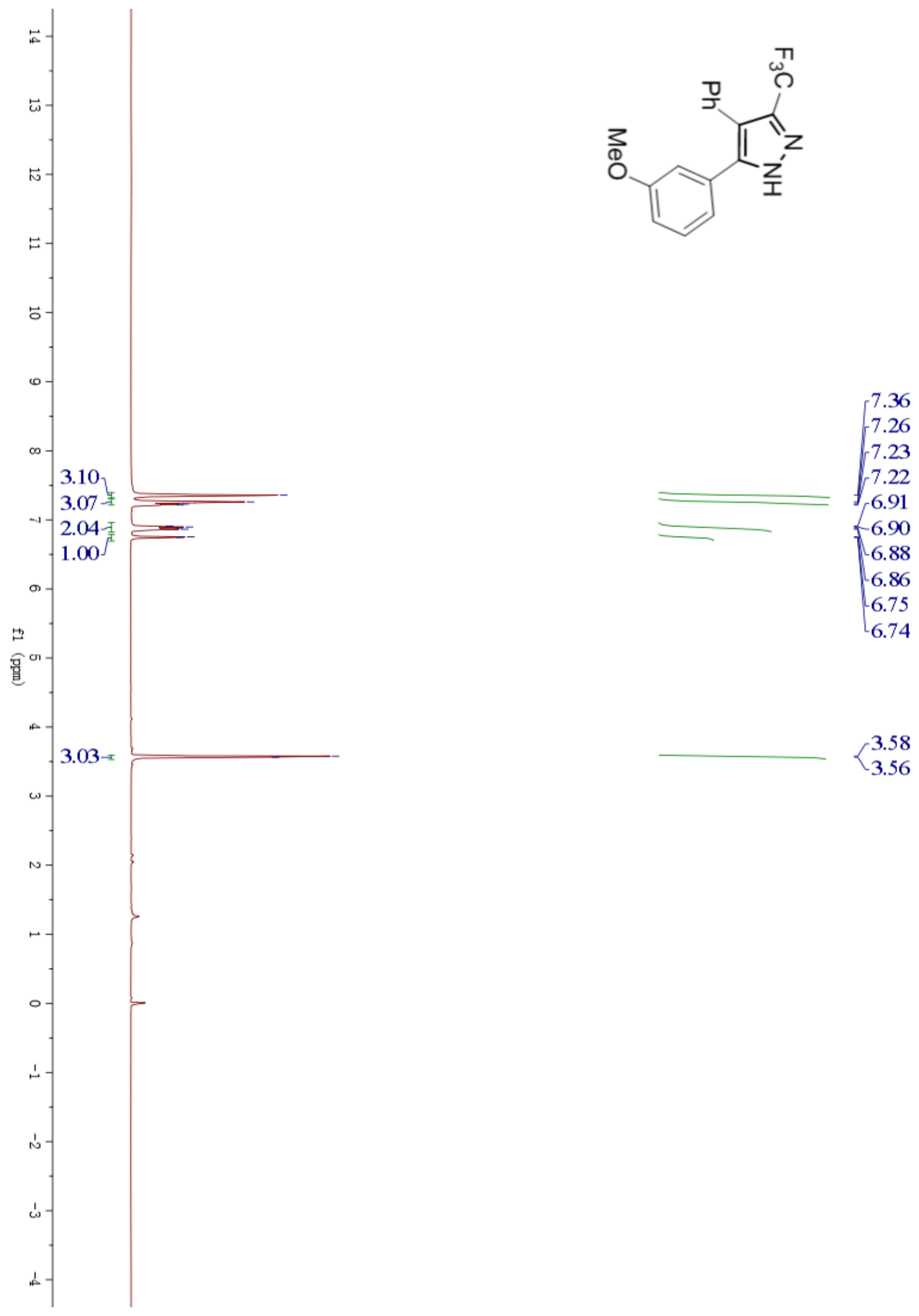


${ }^{13} \mathrm{C}$ NMR (151 MHz, $\mathrm{CDCl}_{3}$ ) spectrum of $\mathbf{5 b}$

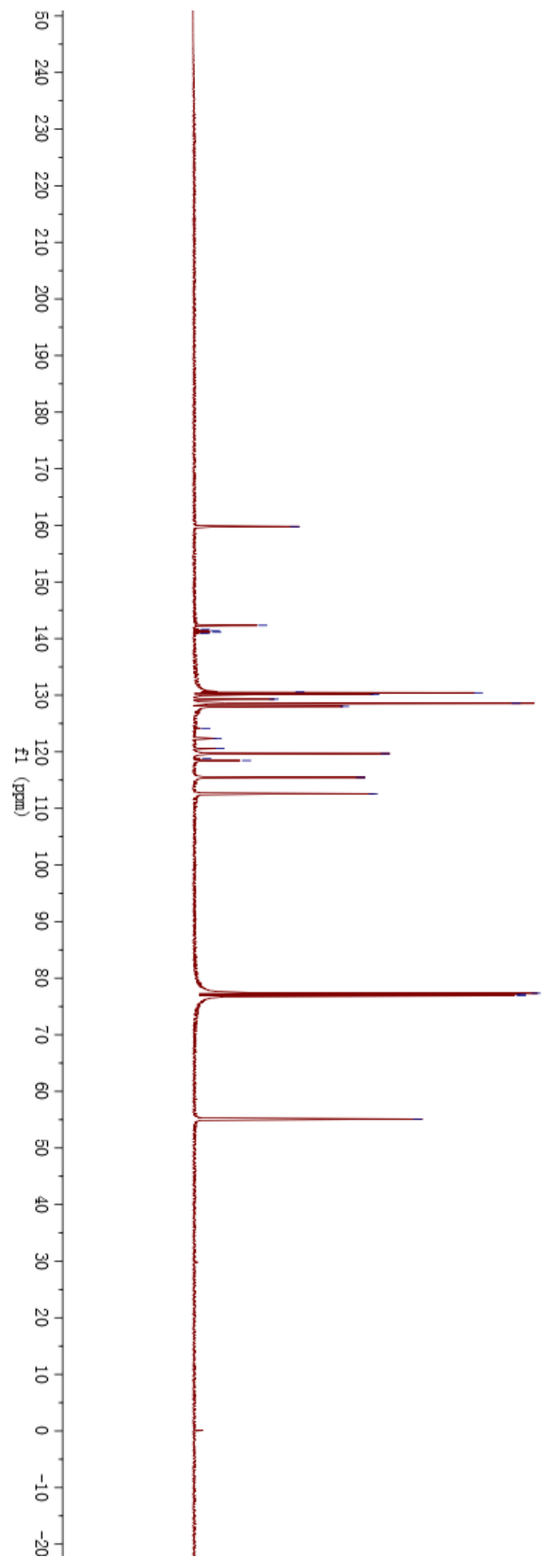

159.78

142.34

$-141.59$

141.35

141.11

140.87

130.55

130.42

130.16

129.28

128.55

128.01

$-124.13$

122.34

120.56

119.67

$-118.77$

$-118.45$

$-115.46$

112.58

77.33

$-77.12$

76.91

$-55.10$ 
${ }^{19} \mathrm{~F} \mathrm{NMR}\left(565 \mathrm{MHz}, \mathrm{CDCl}_{3}\right)$ spectrum of $\mathbf{5 b}$

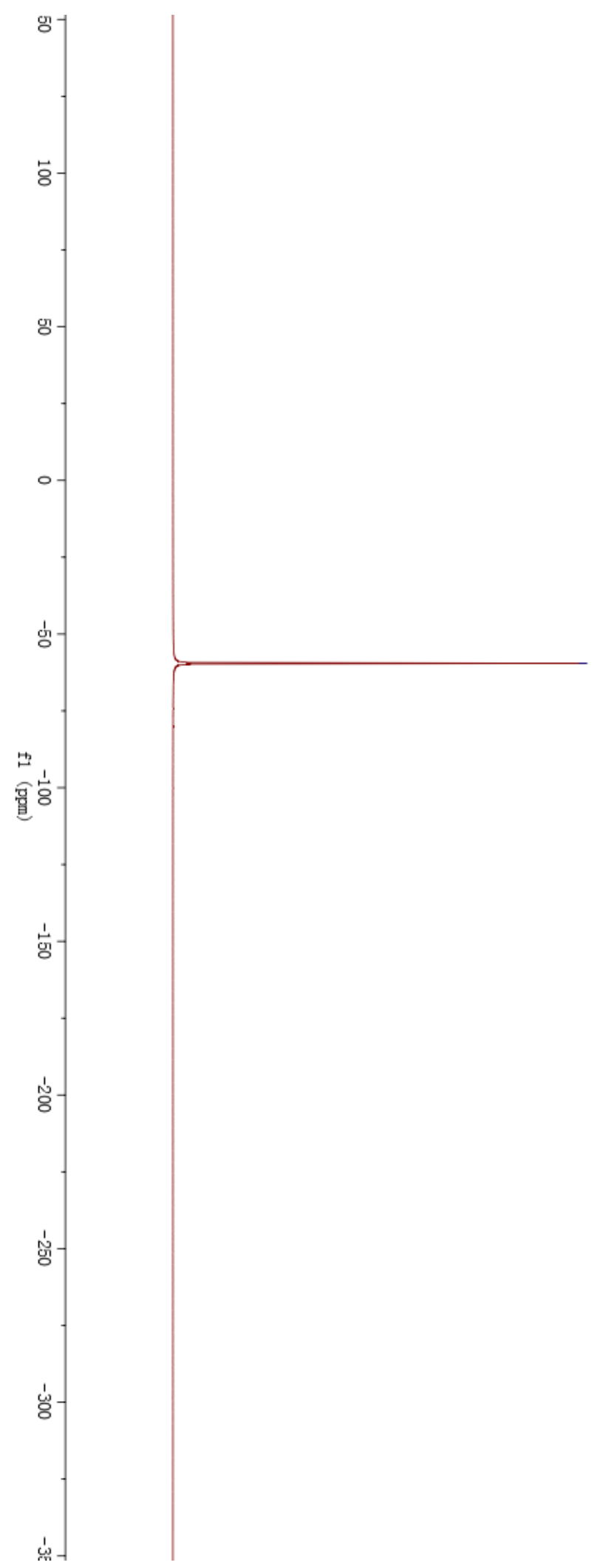


${ }^{1} \mathrm{H}$ NMR $\left(600 \mathrm{MHz}, \mathrm{CDCl}_{3}\right)$ spectrum of $\mathbf{5 c}$

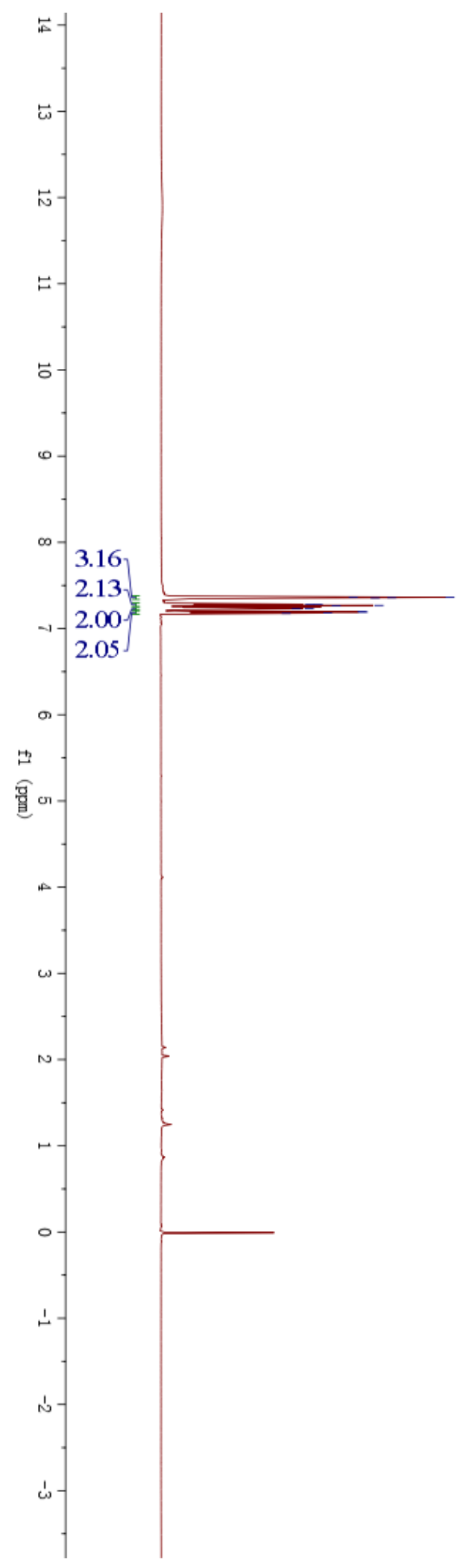

7.37
7.36 $-7.36$ 7.36 7.28 7.28 7.27 7.27

7.23

7.23

7.19

7.19

$-7.18$

7.18 
${ }^{13} \mathrm{C} \mathrm{NMR}\left(151 \mathrm{MHz}, \mathrm{CDCl}_{3}\right)$ spectrum of $\mathbf{5 c}$

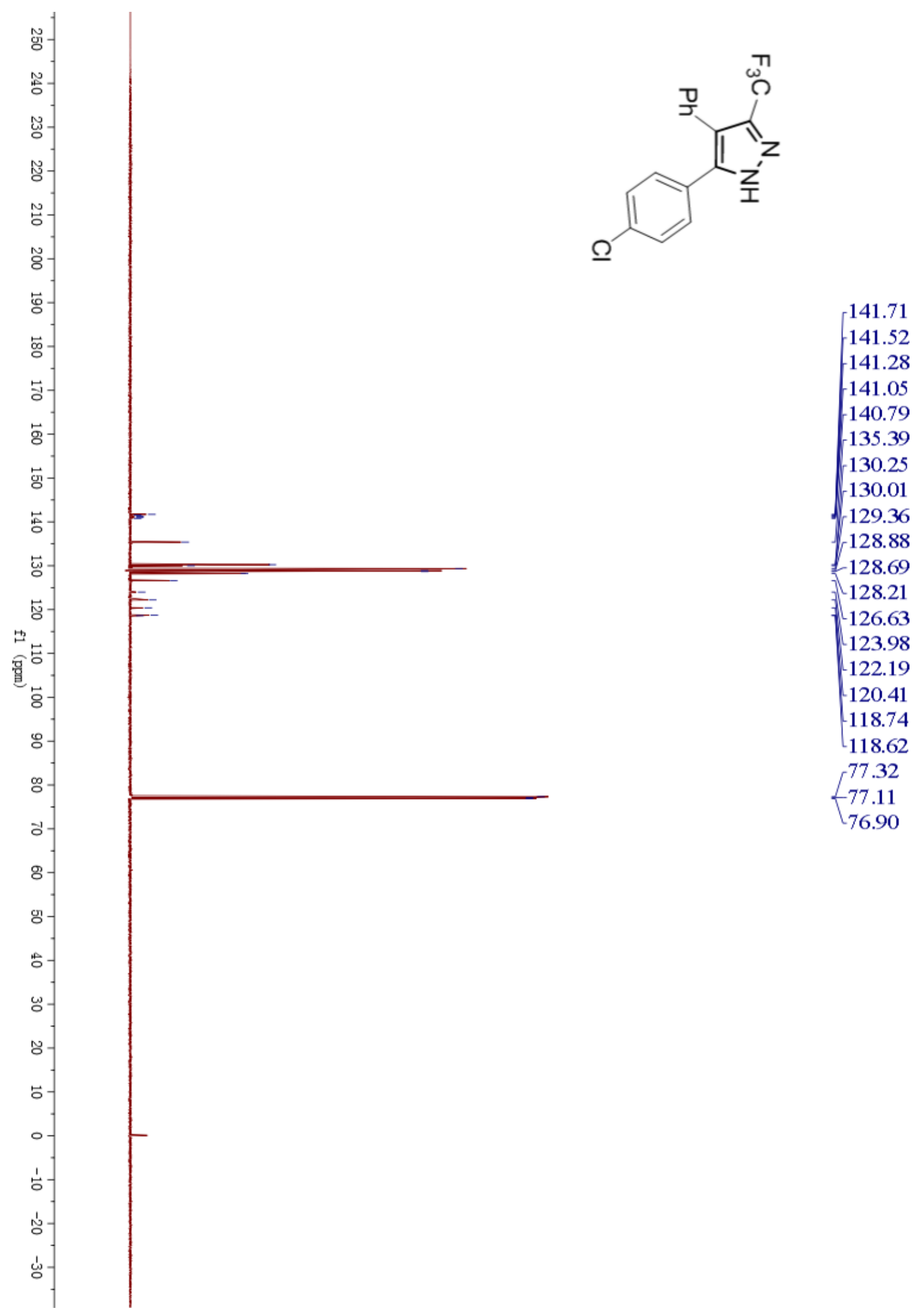


${ }^{19} \mathrm{~F}$ NMR $\left(565 \mathrm{MHz}, \mathrm{CDCl}_{3}\right)$ spectrum of $\mathbf{5 c}$

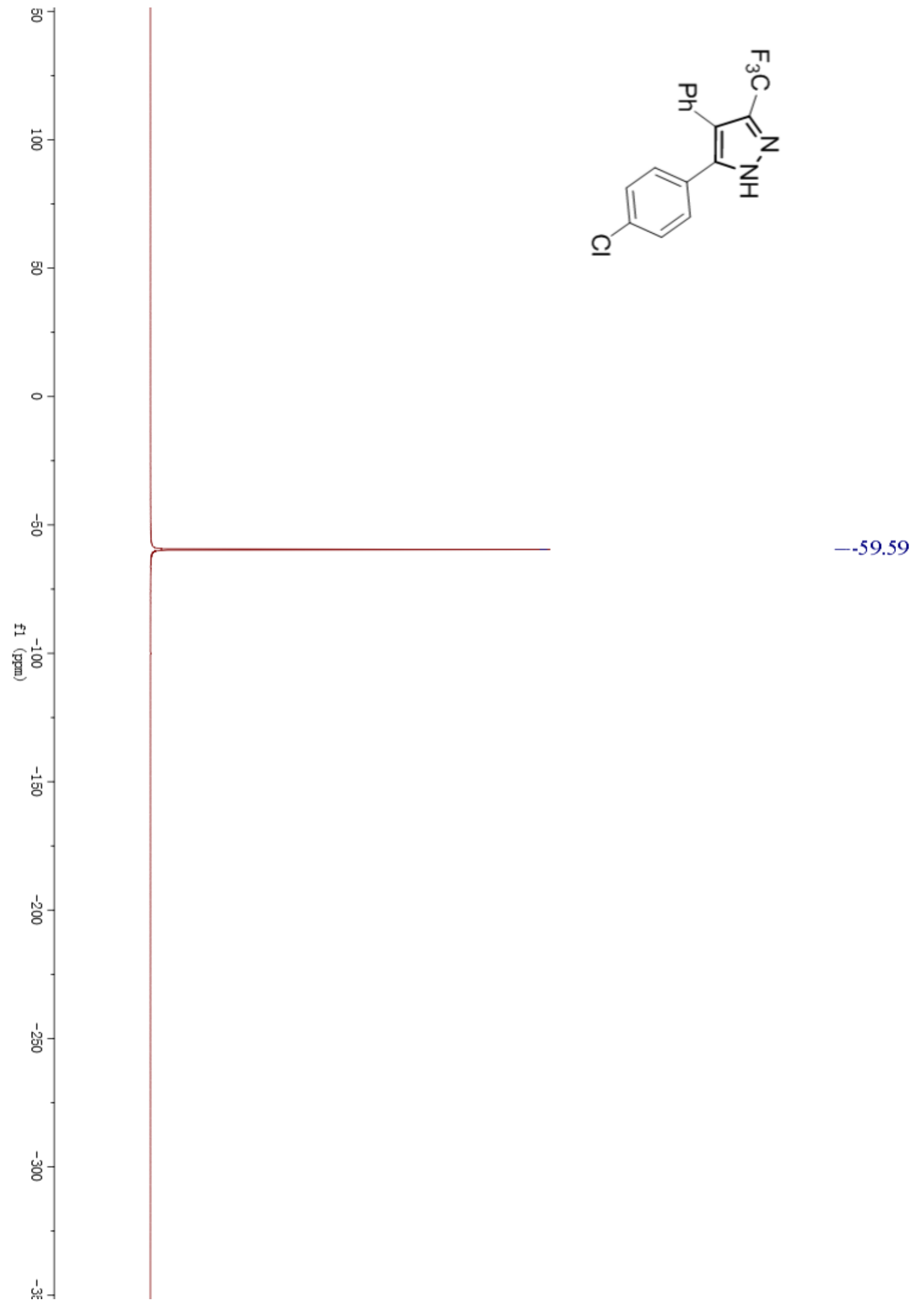


${ }^{1} \mathrm{H}$ NMR (600 MHz, $\left.\mathrm{CDCl}_{3}\right)$ spectrum of $\mathbf{5 d}$

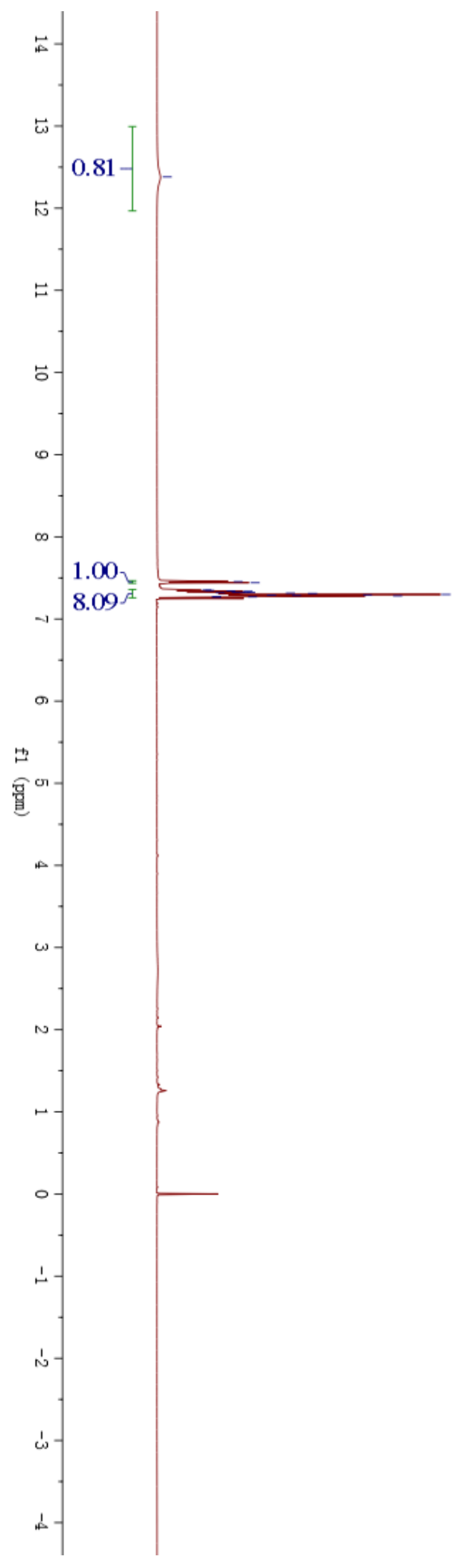

7.45 7.44 $-7.35$ 7.35 7.34 7.34 7.33 7.32

7.32 7.32 7.31 7.31

7.30

7.29 7.29 7.28 7.28 7.27 $-7.27$ 7.26 
${ }^{13} \mathrm{C} \mathrm{NMR}\left(151 \mathrm{MHz}, \mathrm{CDCl}_{3}\right)$ spectrum of $\mathbf{5 d}$

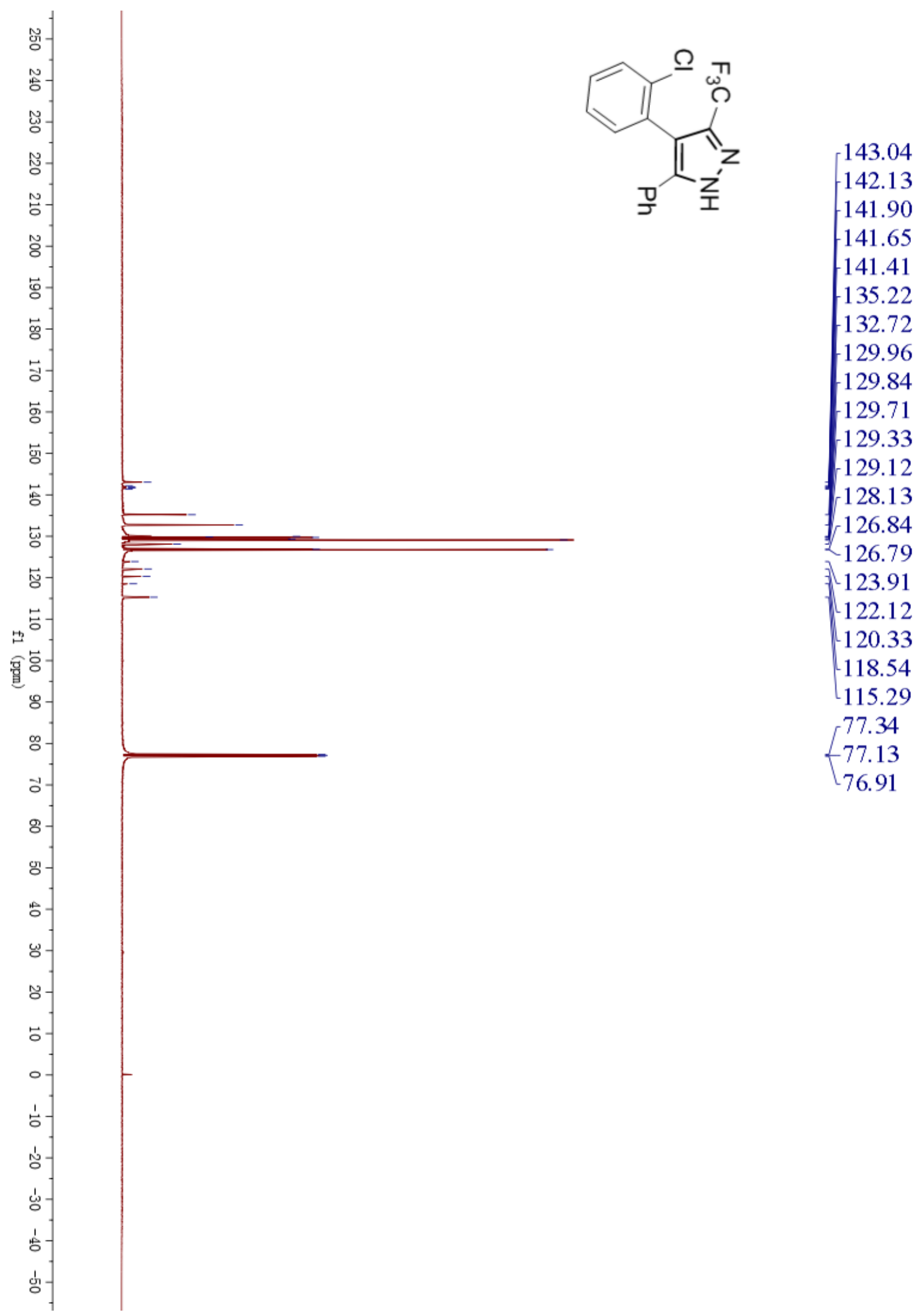


${ }^{19} \mathrm{~F}$ NMR $\left(565 \mathrm{MHz}, \mathrm{CDCl}_{3}\right)$ spectrum of $\mathbf{5 d}$

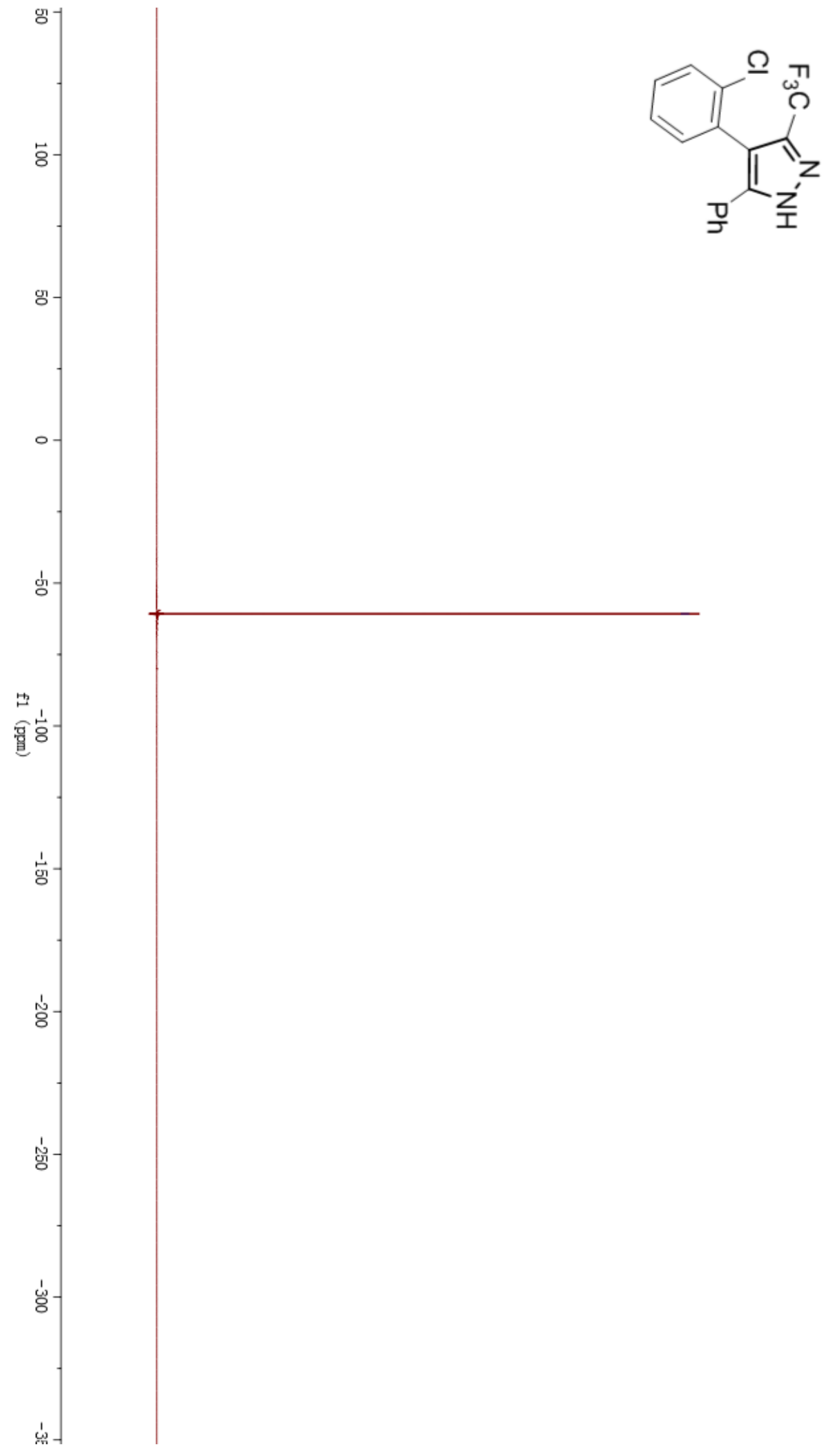


${ }^{1} \mathrm{H}$ NMR (600 MHz, $\mathrm{CDCl}_{3}$ ) spectrum of $\mathbf{5 e}$

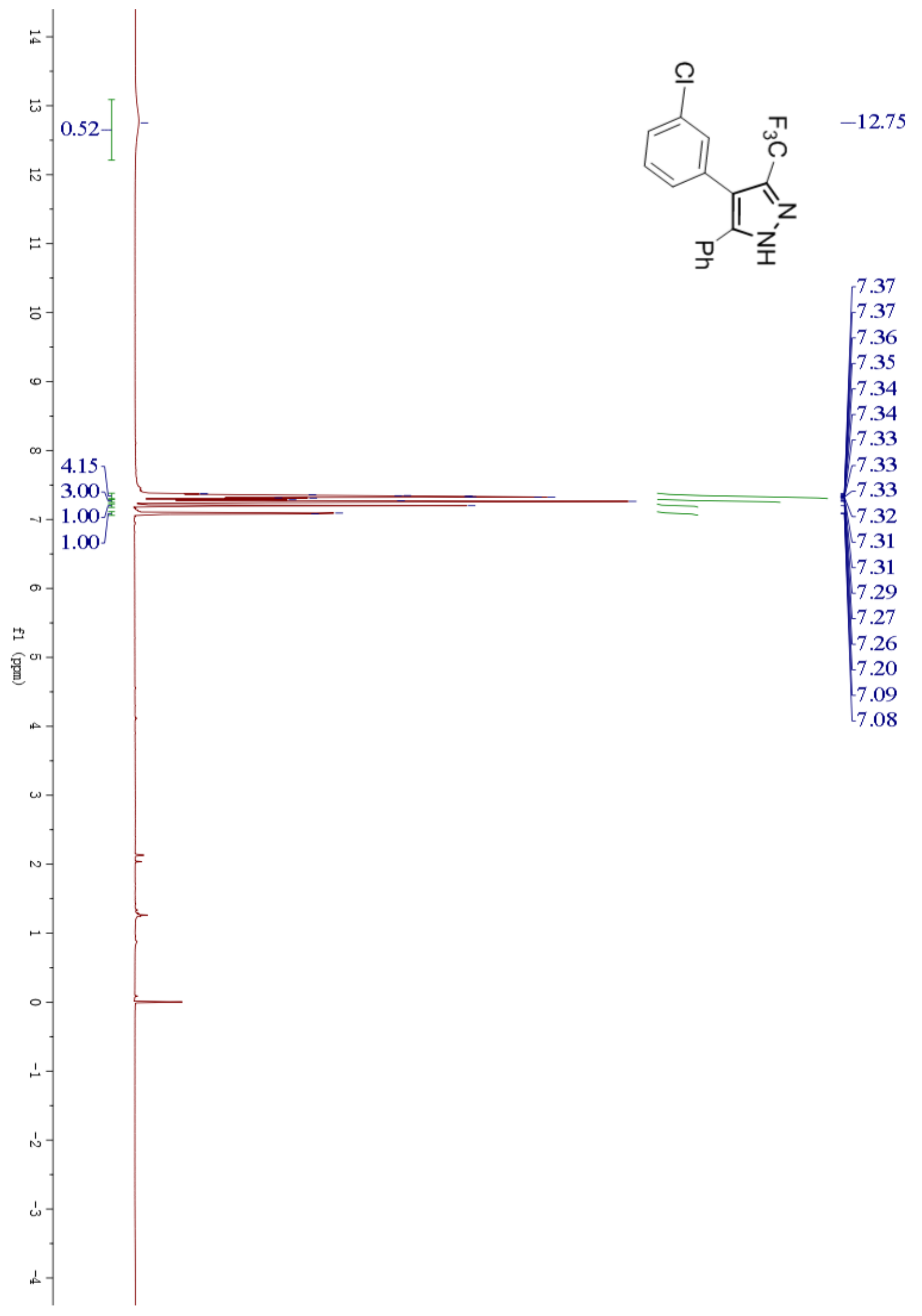


${ }^{13} \mathrm{C} \mathrm{NMR}\left(151 \mathrm{MHz}, \mathrm{CDCl}_{3}\right)$ spectrum of $\mathbf{5 e}$

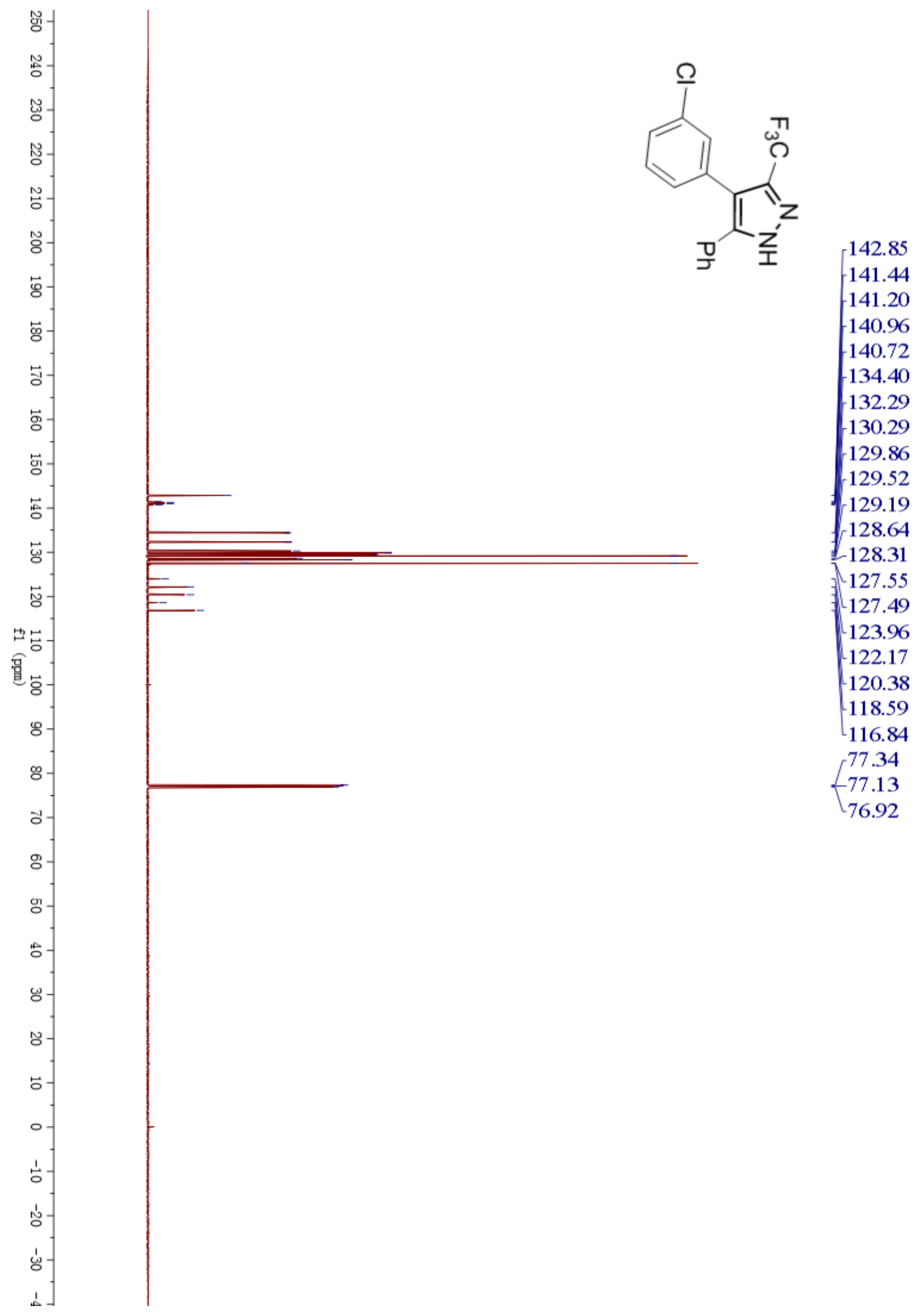


${ }^{19} \mathrm{~F}$ NMR $\left(565 \mathrm{MHz}, \mathrm{CDCl}_{3}\right)$ spectrum of $\mathbf{5 e}$

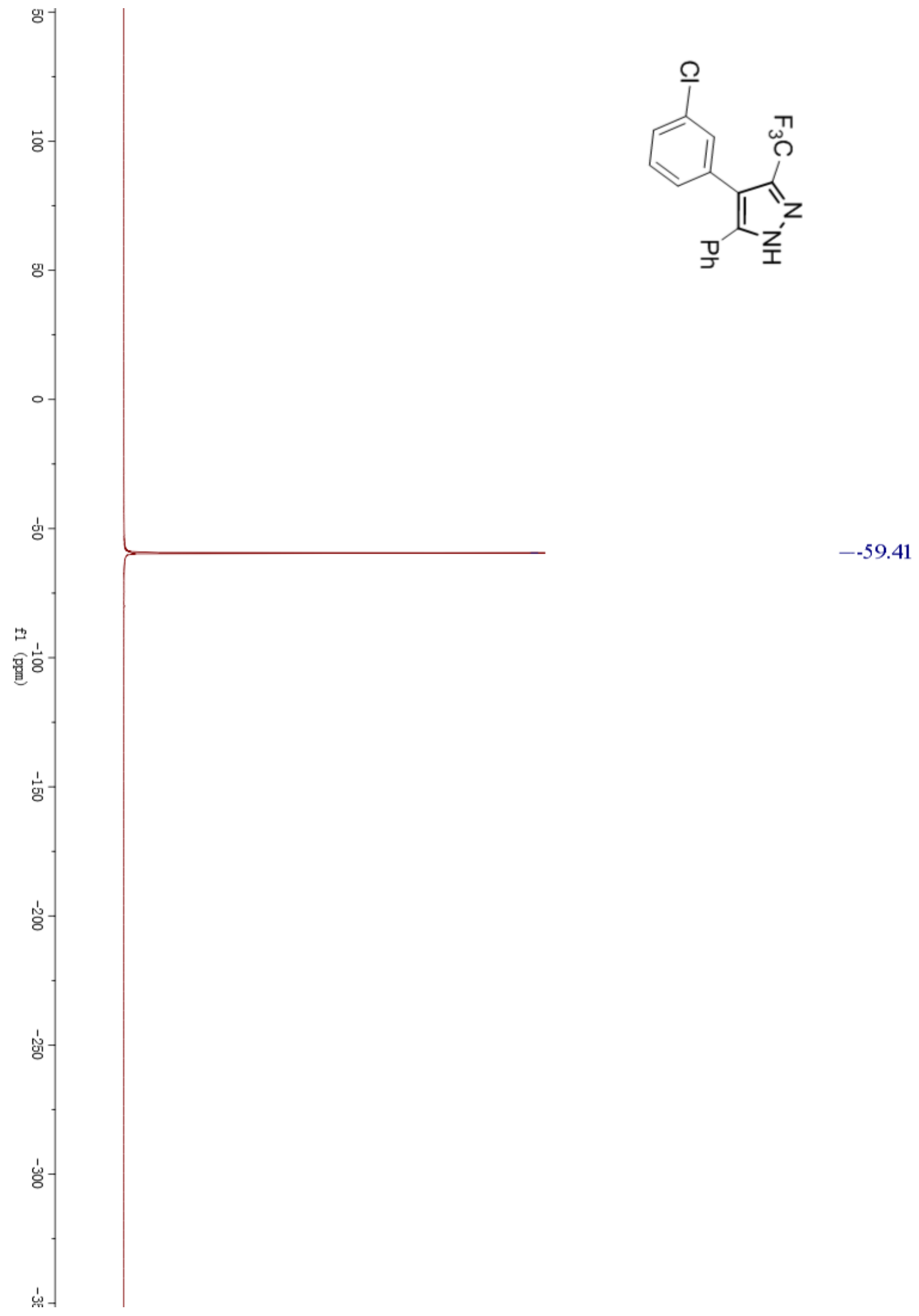


${ }^{1} \mathrm{H}$ NMR $\left(600 \mathrm{MHz}, \mathrm{CDCl}_{3}\right)$ spectrum of $\mathbf{5 f}$

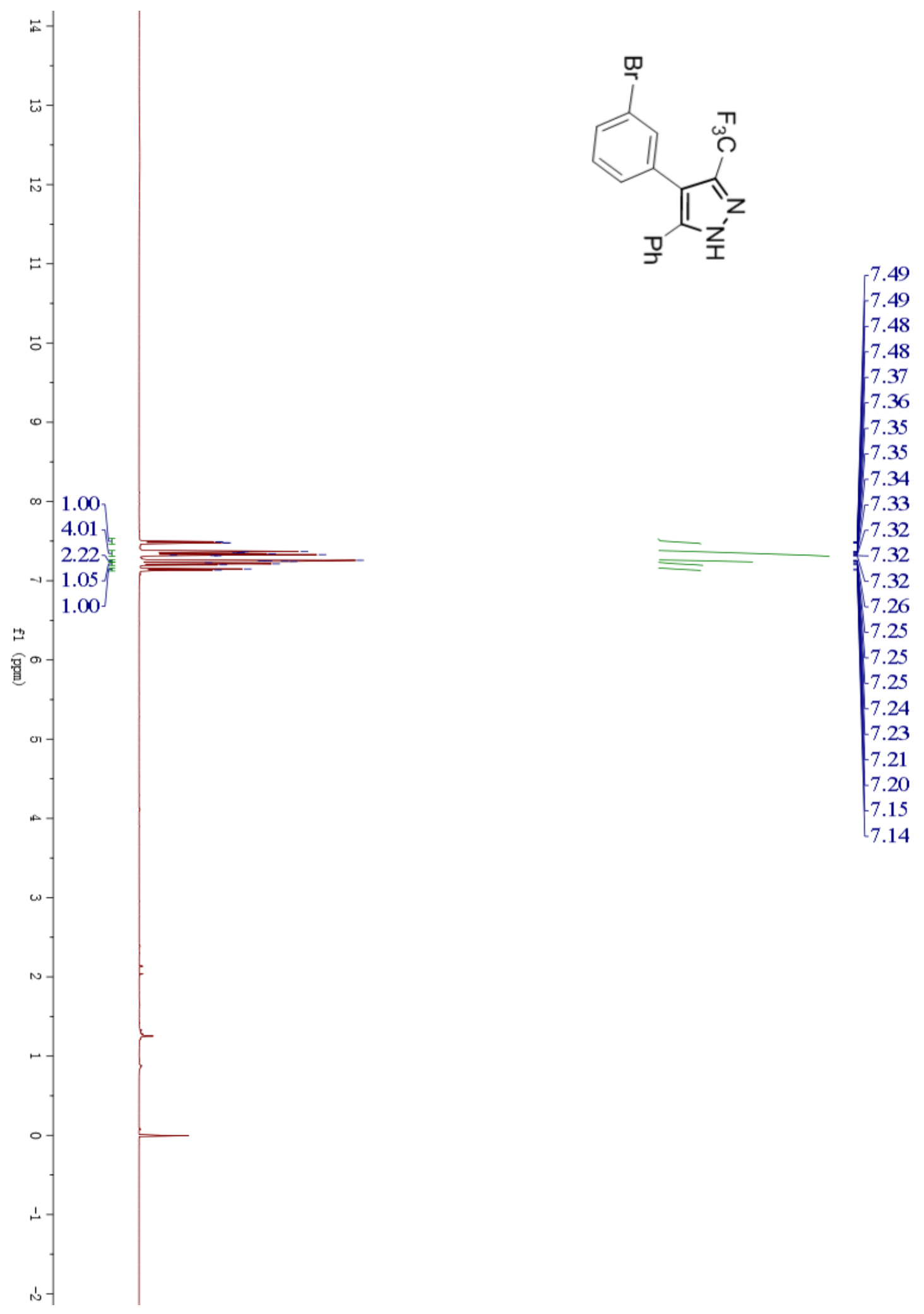


${ }^{13} \mathrm{C}$ NMR $\left(151 \mathrm{MHz}, \mathrm{CDCl}_{3}\right)$ spectrum of $\mathbf{5 f}$

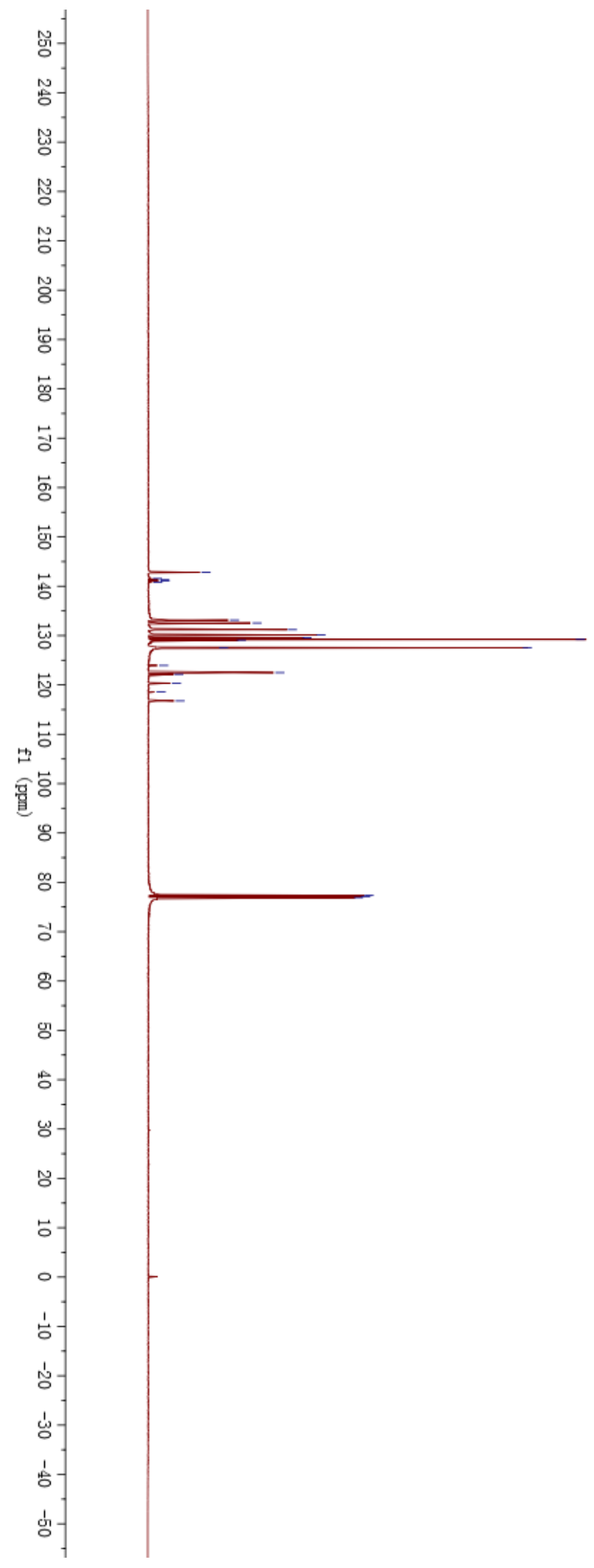<smiles></smiles>

142.80

$-141.55$

141.31

141.07

140.83

133.14

132.55

$-131.20$

130.10

$-129.54$

129.21

129.09

127.56

127.52

123.95

122.50

122.16

120.37

118.58

116.76

77.34

$-77.12$

76.91 
${ }^{19} \mathrm{~F}$ NMR (565 MHz, $\mathrm{CDCl}_{3}$ ) spectrum of $\mathbf{5 f}$

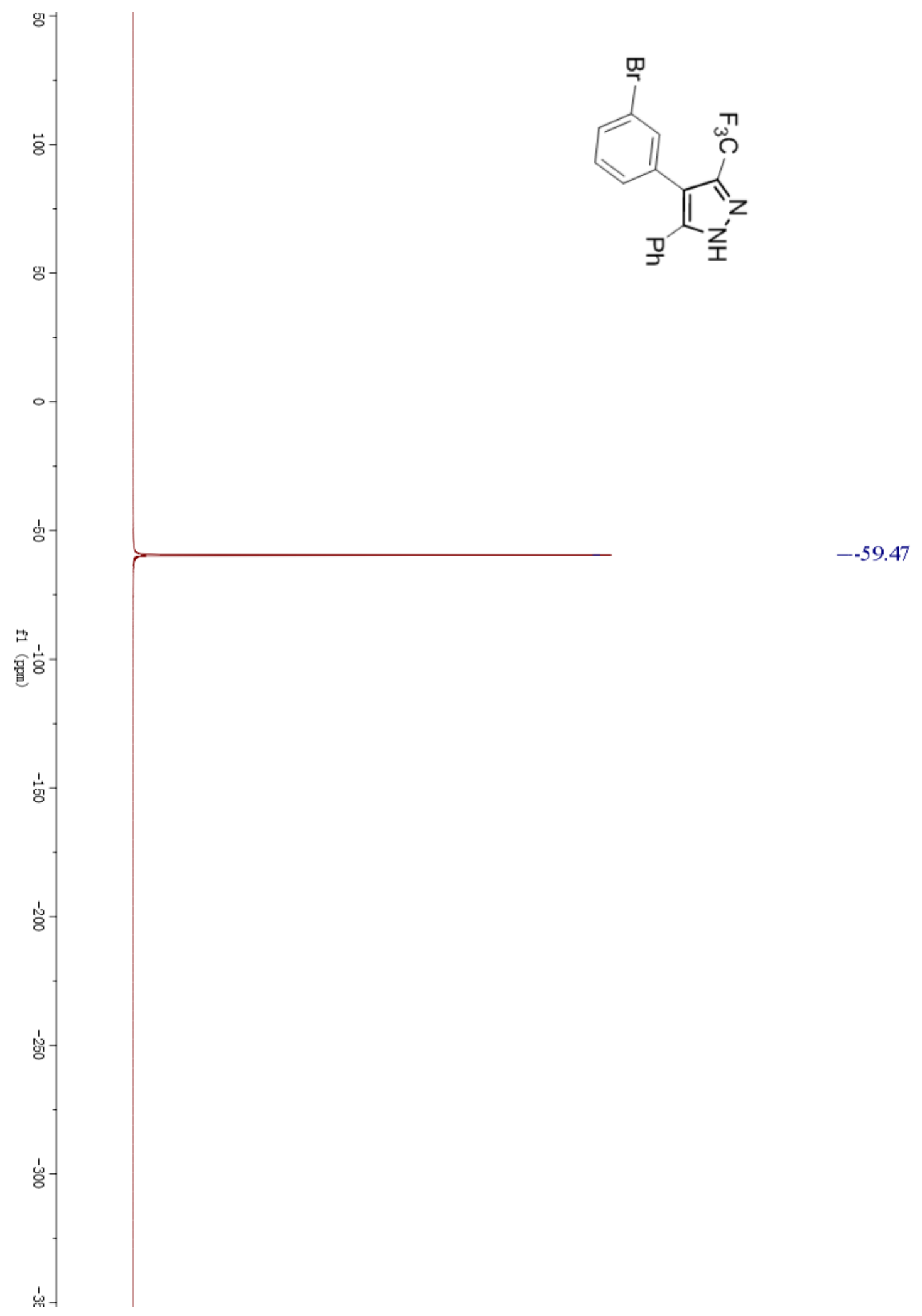


${ }^{1} \mathrm{H}$ NMR (600 MHz, DMSO- $\left.d_{6}\right)$ spectrum of $\mathbf{5 g}$

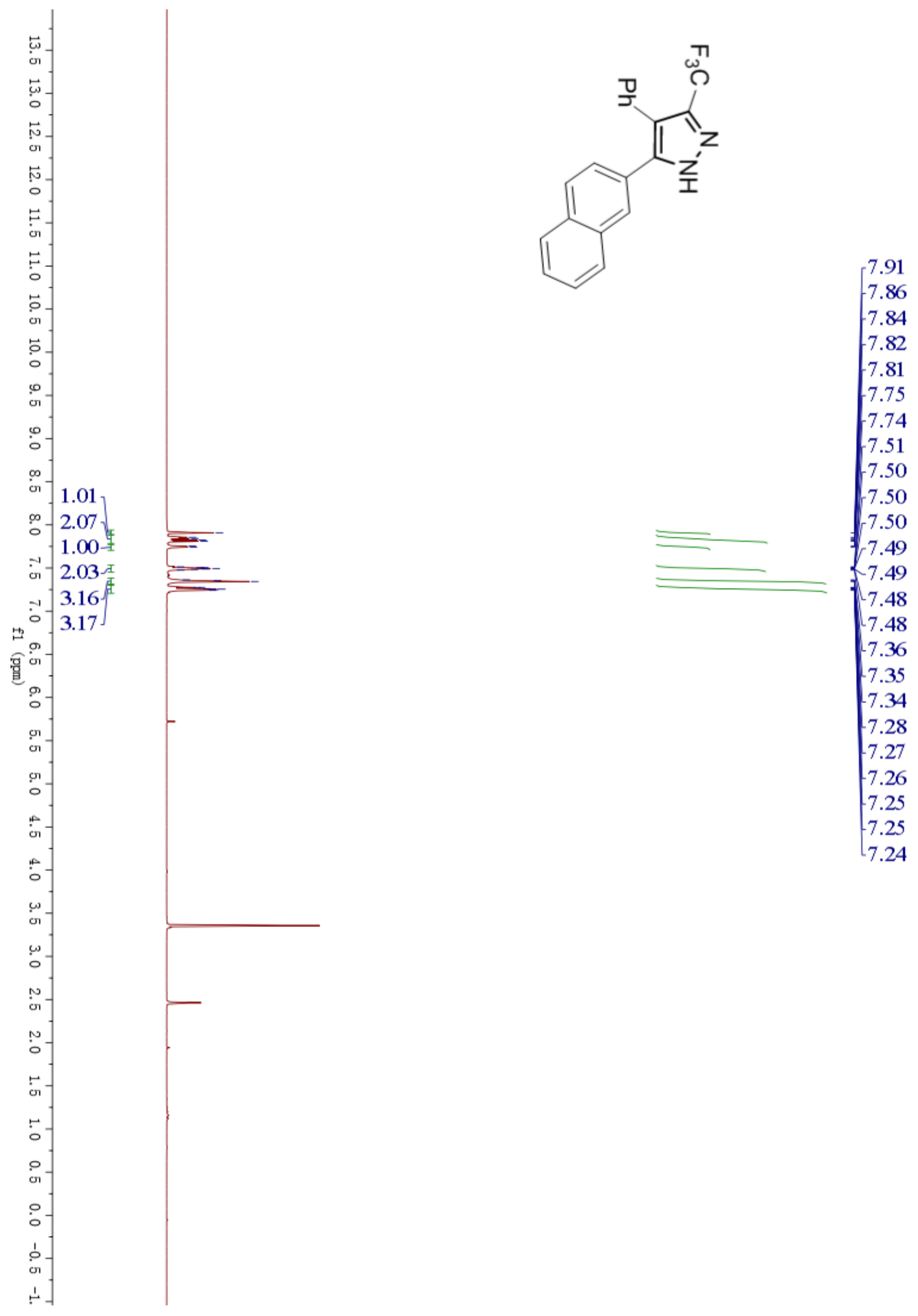


${ }^{13} \mathrm{C}$ NMR $\left(151 \mathrm{MHz}\right.$, DMSO- $\left.d_{6}\right)$ spectrum of $\mathbf{5 g}$

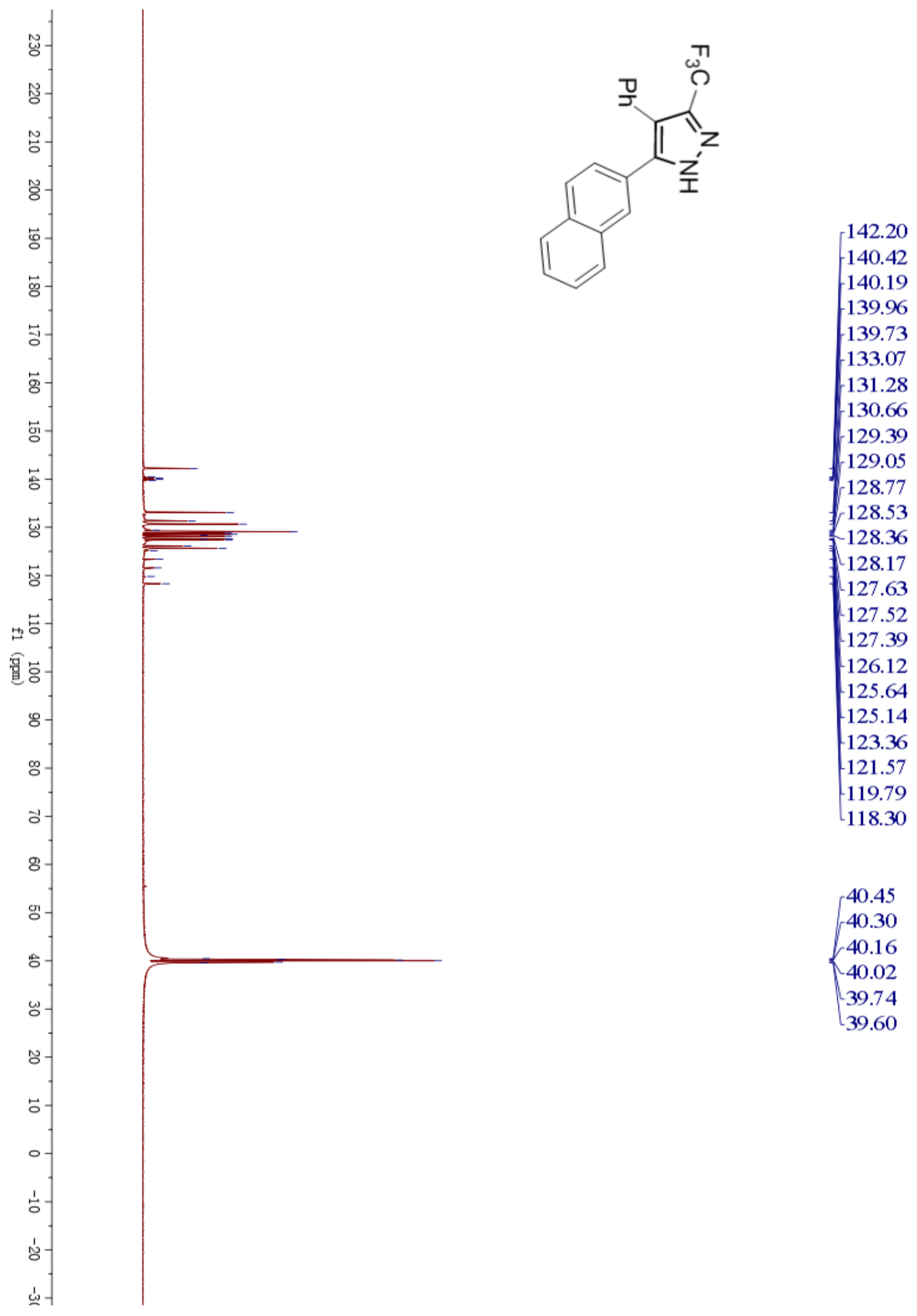


${ }^{19}$ F NMR (565 MHz, DMSO-d6) spectrum of $\mathbf{5 g}$

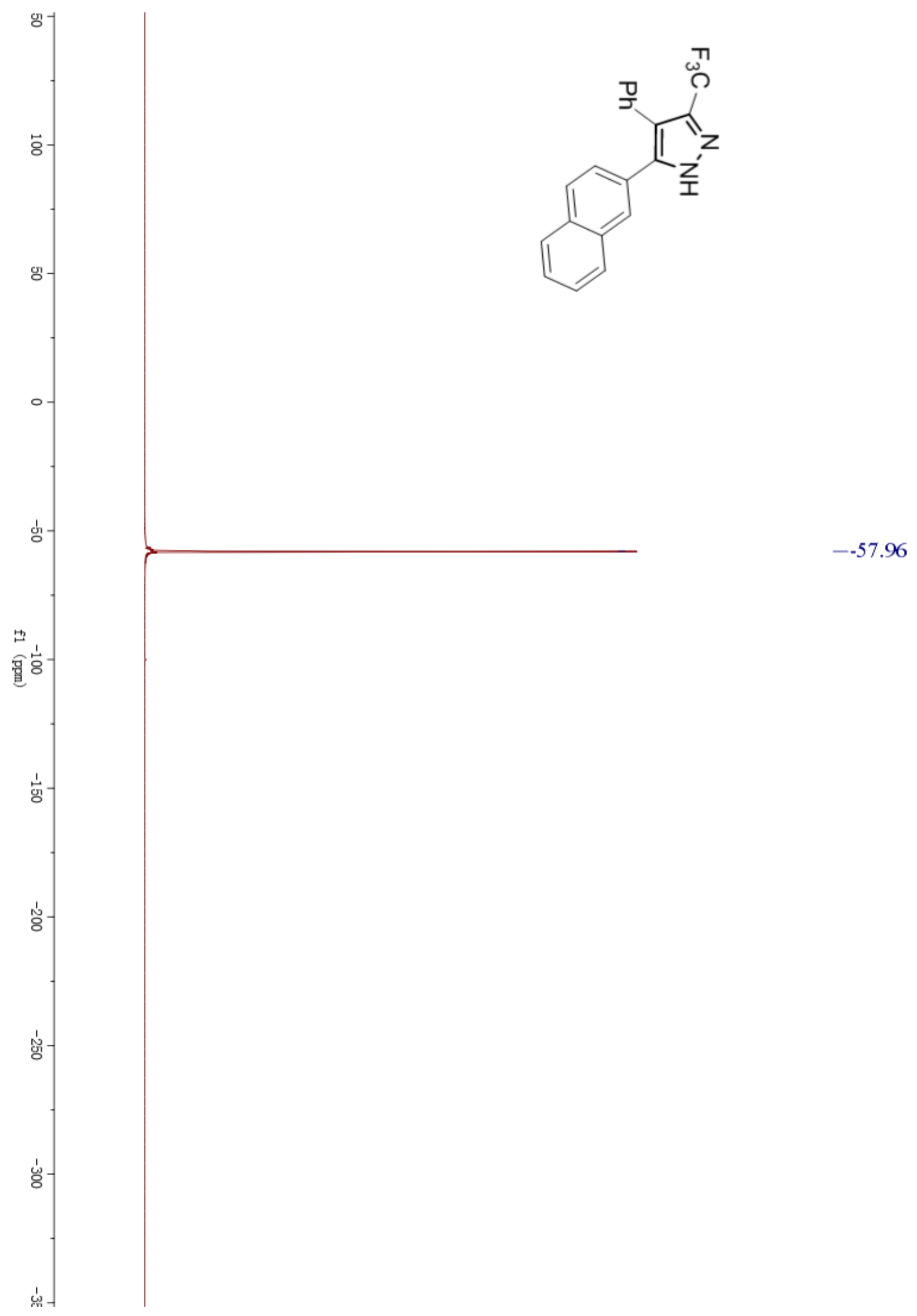


${ }^{1} \mathrm{H}$ NMR $\left(600 \mathrm{MHz}, \mathrm{CDCl}_{3}\right)$ spectrum of $\mathbf{6 a}$

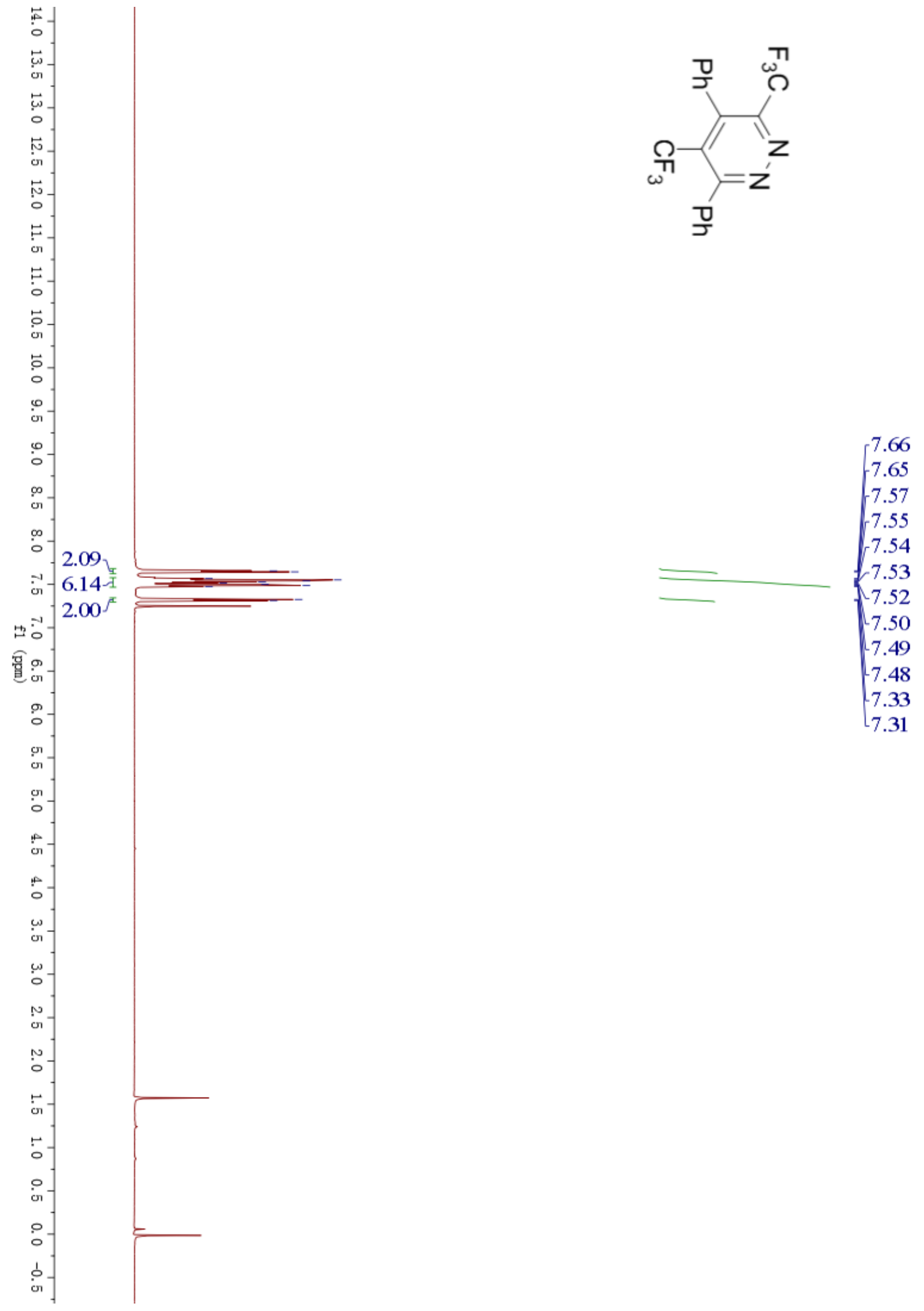


${ }^{13} \mathrm{C} \mathrm{NMR}\left(151 \mathrm{MHz}, \mathrm{CDCl}_{3}\right)$ spectrum of $\mathbf{6 a}$
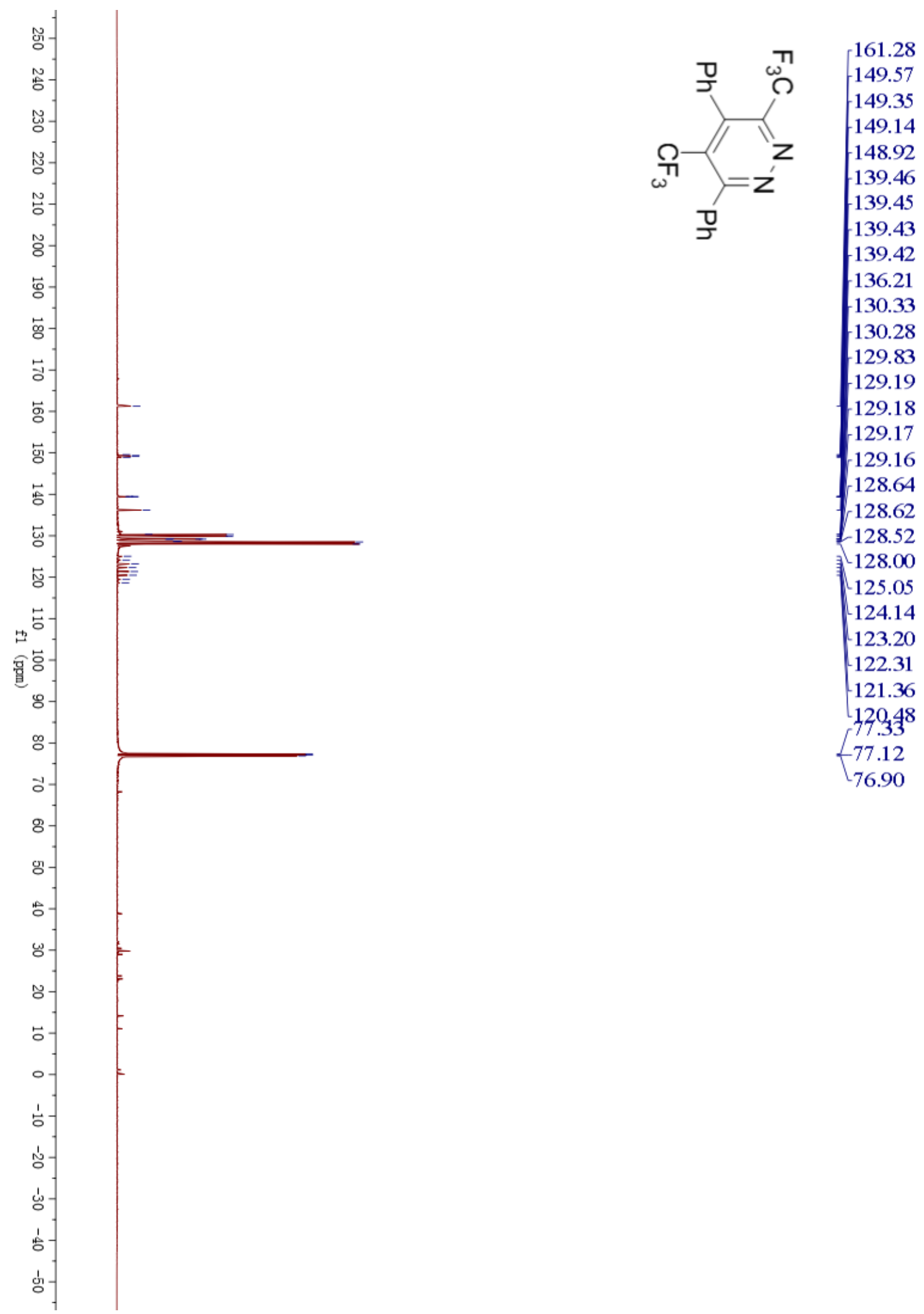
${ }^{19} \mathrm{~F} \mathrm{NMR}\left(565 \mathrm{MHz}, \mathrm{CDCl}_{3}\right)$ spectrum of $\mathbf{6 a}$

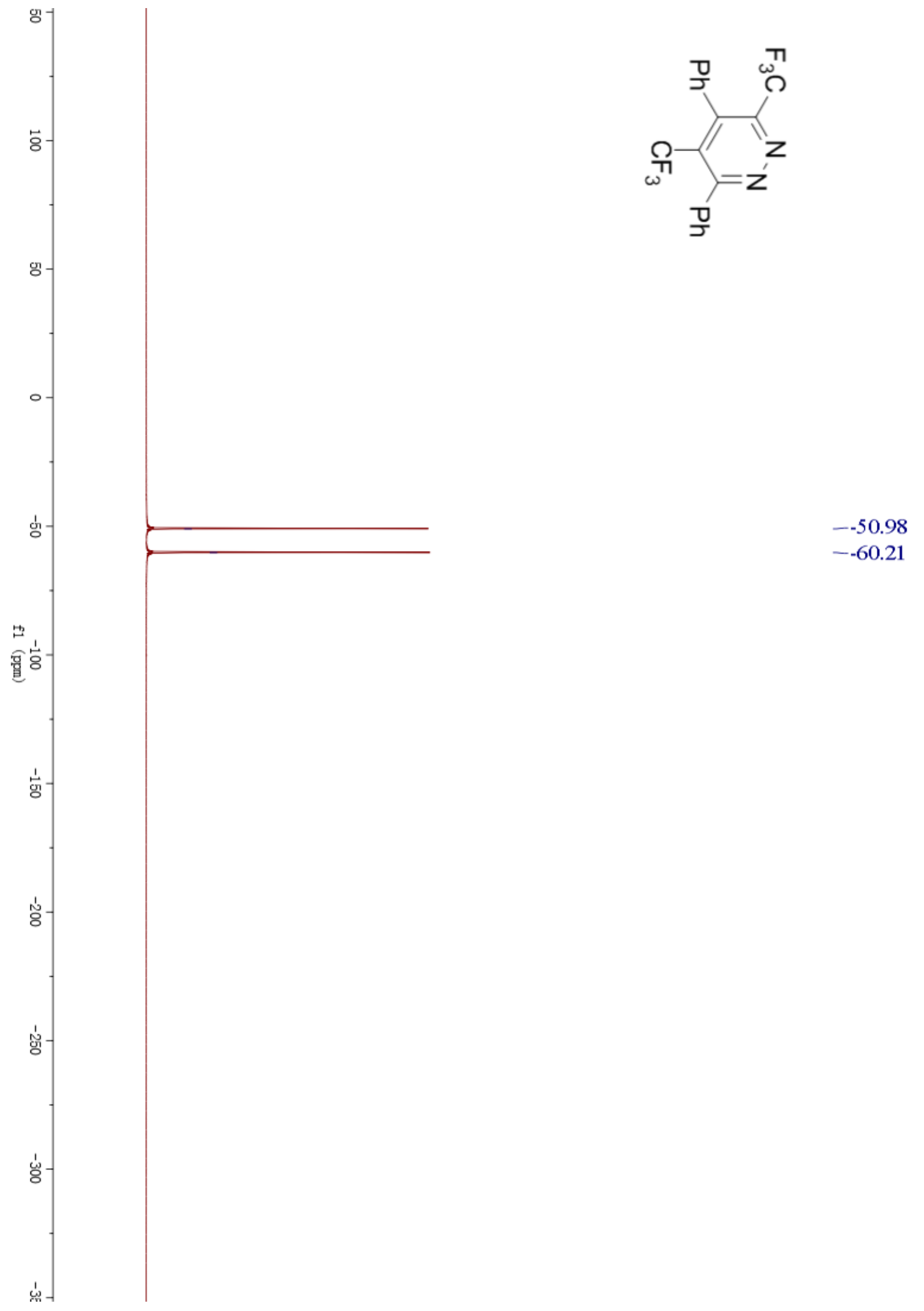


${ }^{1} \mathrm{H}$ NMR $\left(600 \mathrm{MHz}, \mathrm{CDCl}_{3}\right)$ spectrum of $\mathbf{6 b}$
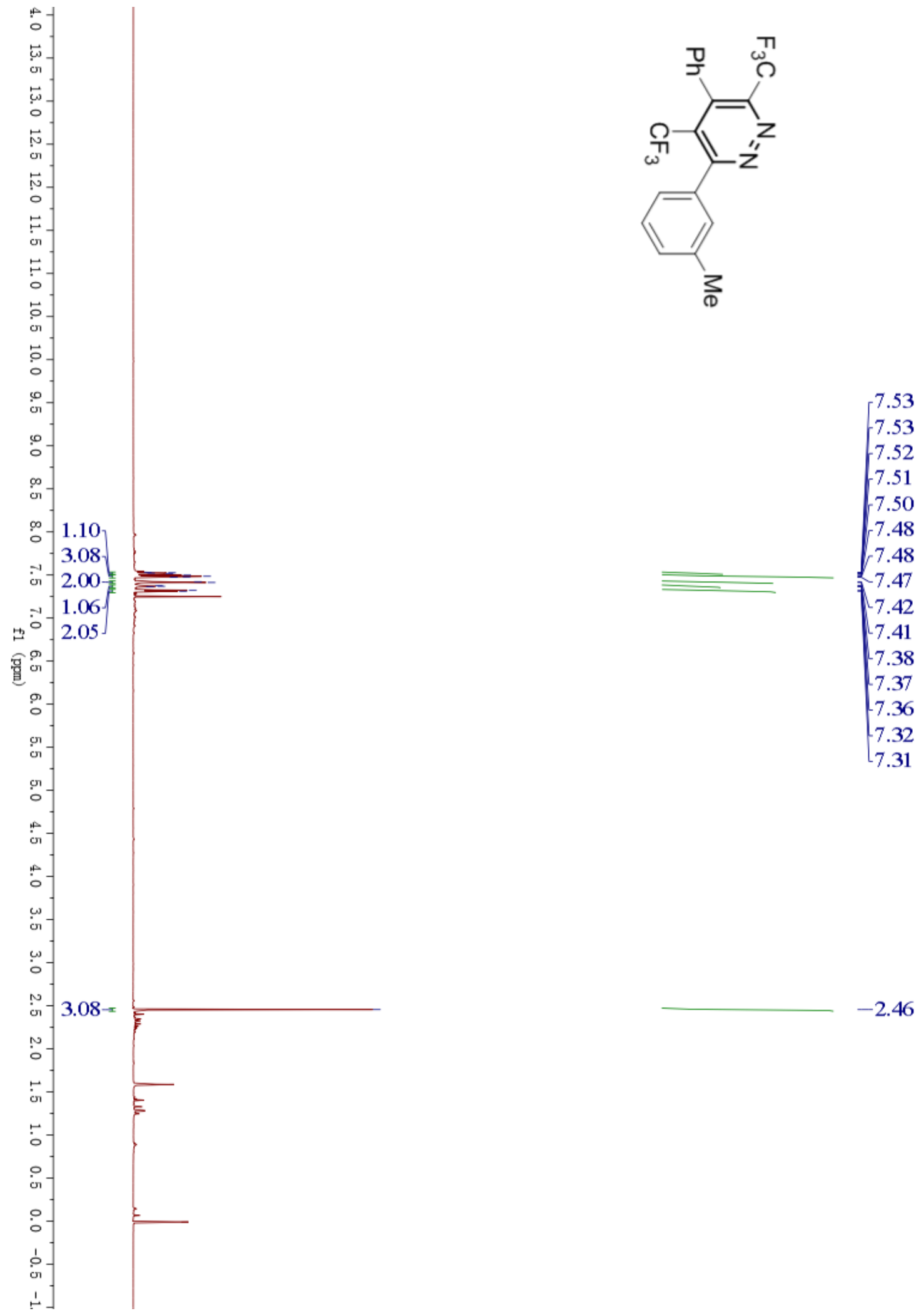

$-2.46$ 
${ }^{13} \mathrm{C}$ NMR (151 MHz, $\mathrm{CDCl}_{3}$ ) spectrum of $\mathbf{6 b}$

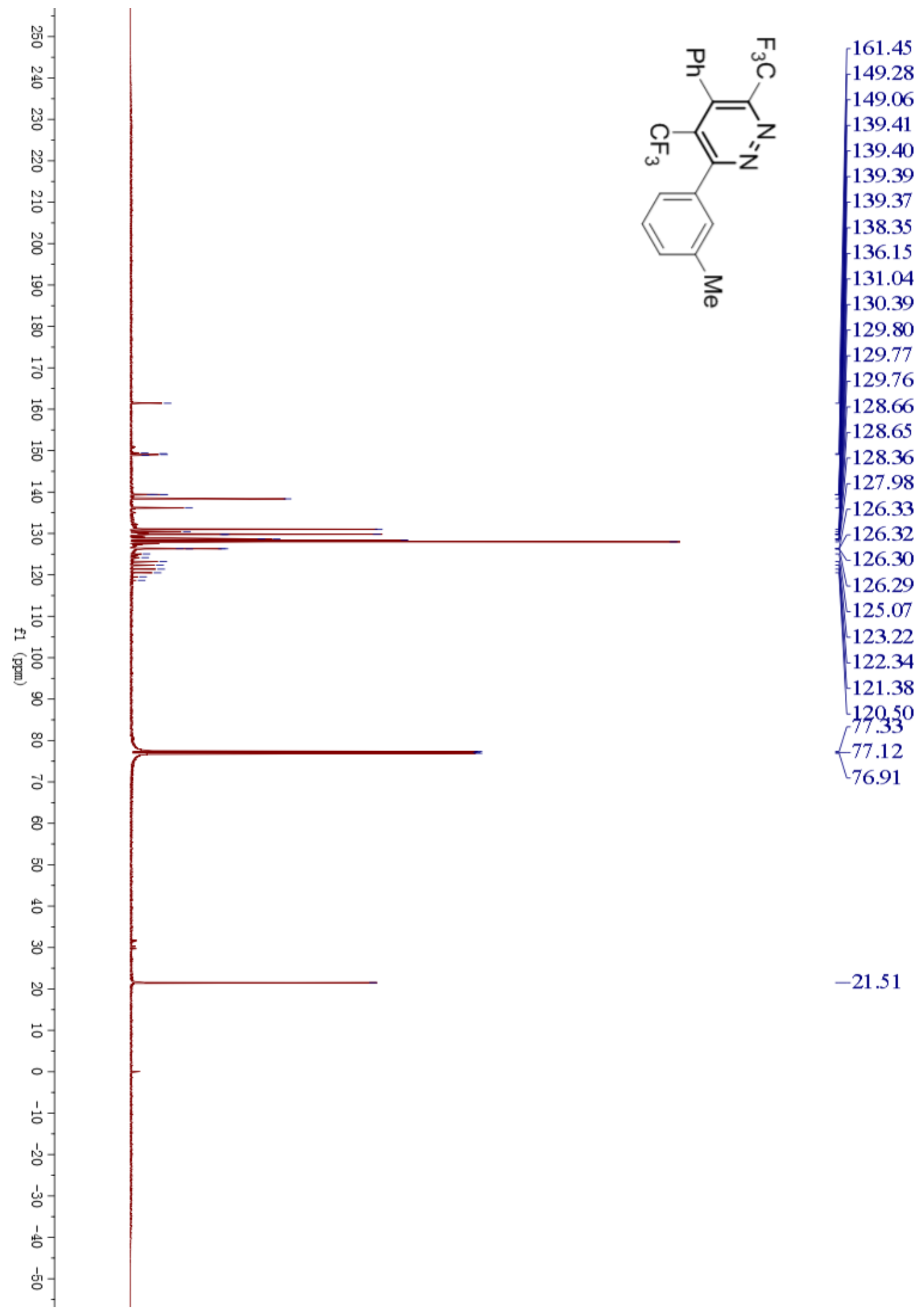


${ }^{19} \mathrm{~F}$ NMR $\left(565 \mathrm{MHz}, \mathrm{CDCl}_{3}\right)$ spectrum of $\mathbf{6 b}$

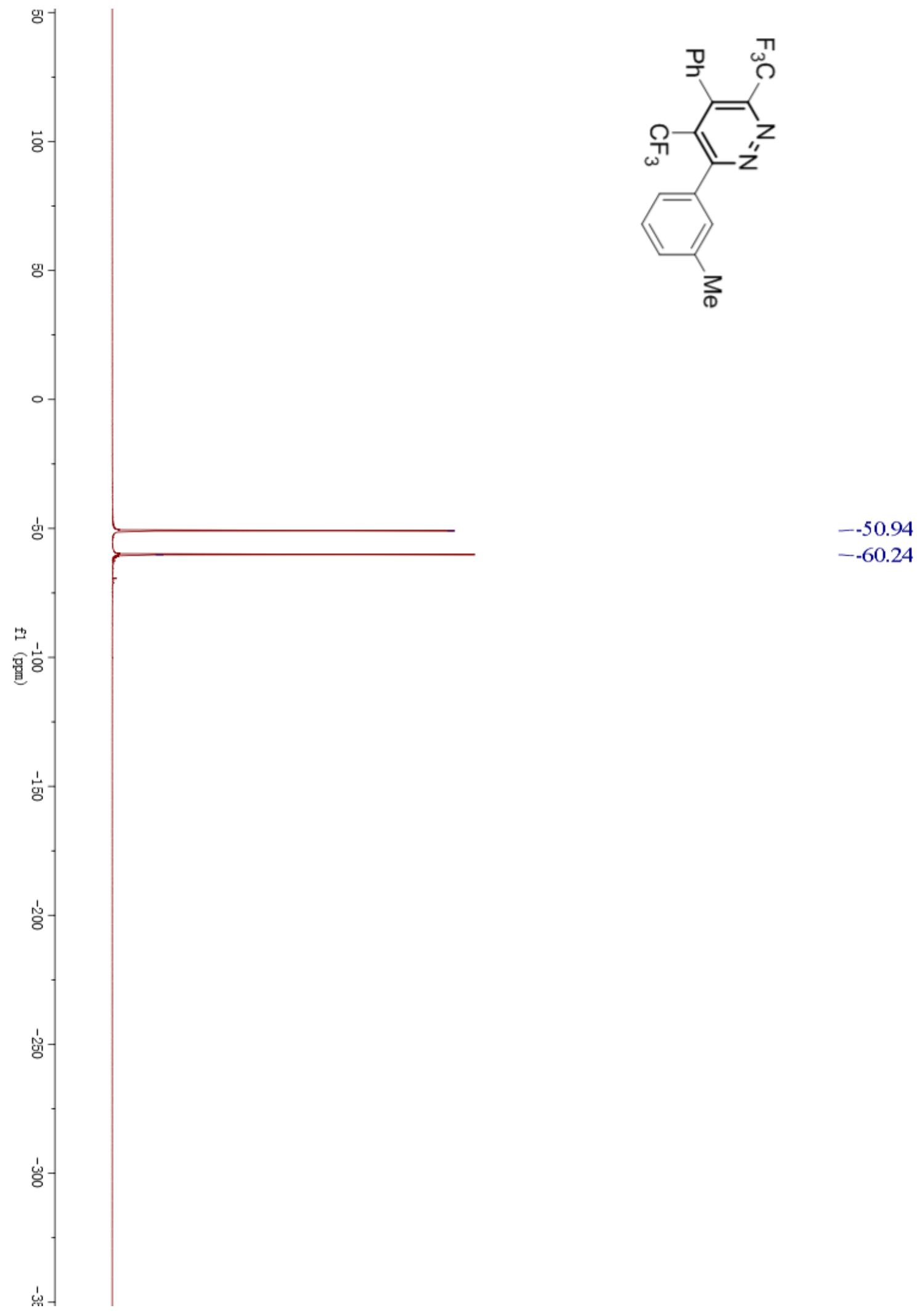


${ }^{1} \mathrm{H}$ NMR $\left(600 \mathrm{MHz}, \mathrm{CDCl}_{3}\right)$ spectrum of $\mathbf{6 c}$
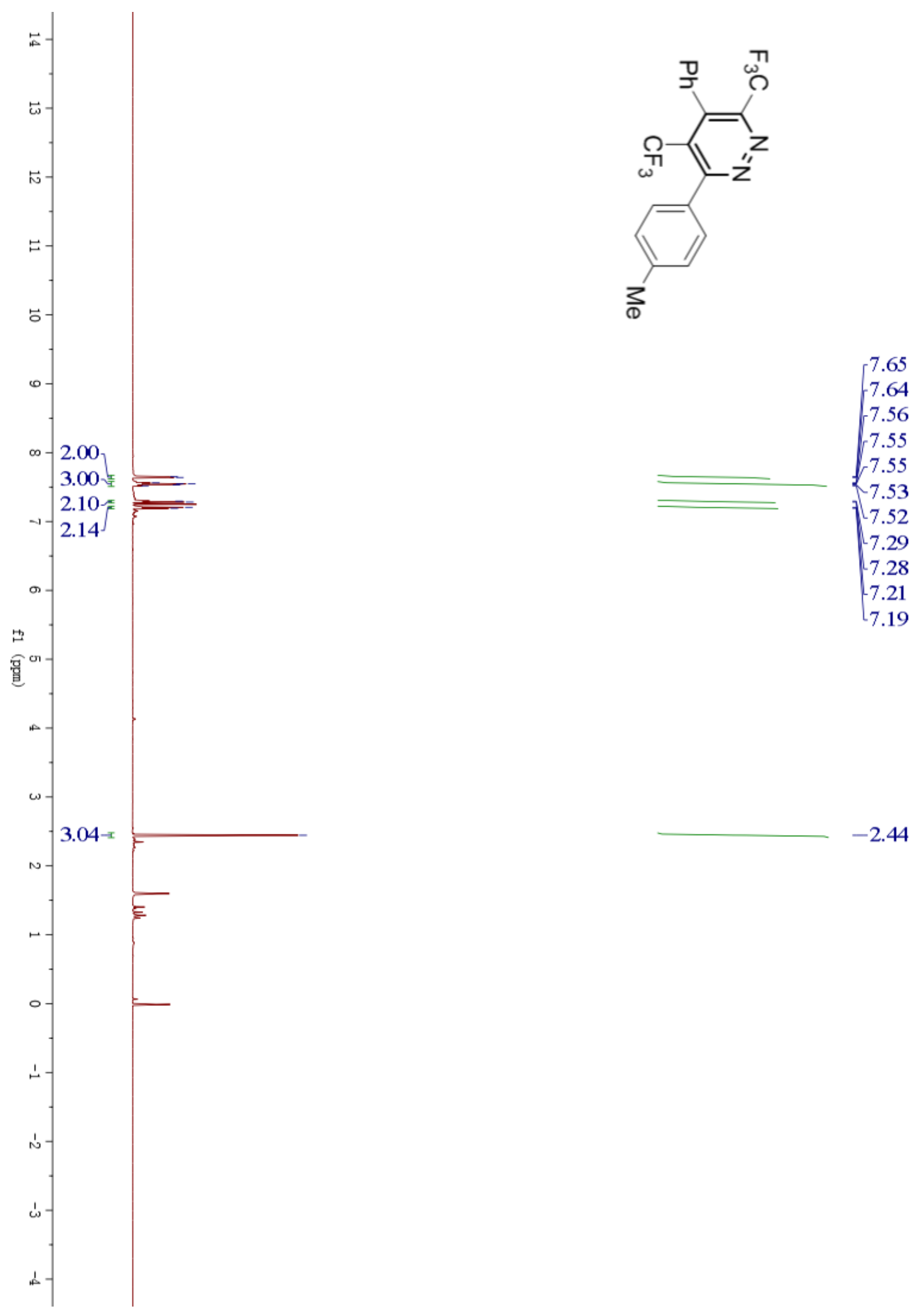
${ }^{13} \mathrm{C} \mathrm{NMR}\left(151 \mathrm{MHz}, \mathrm{CDCl}_{3}\right)$ spectrum of $\mathbf{6 c}$

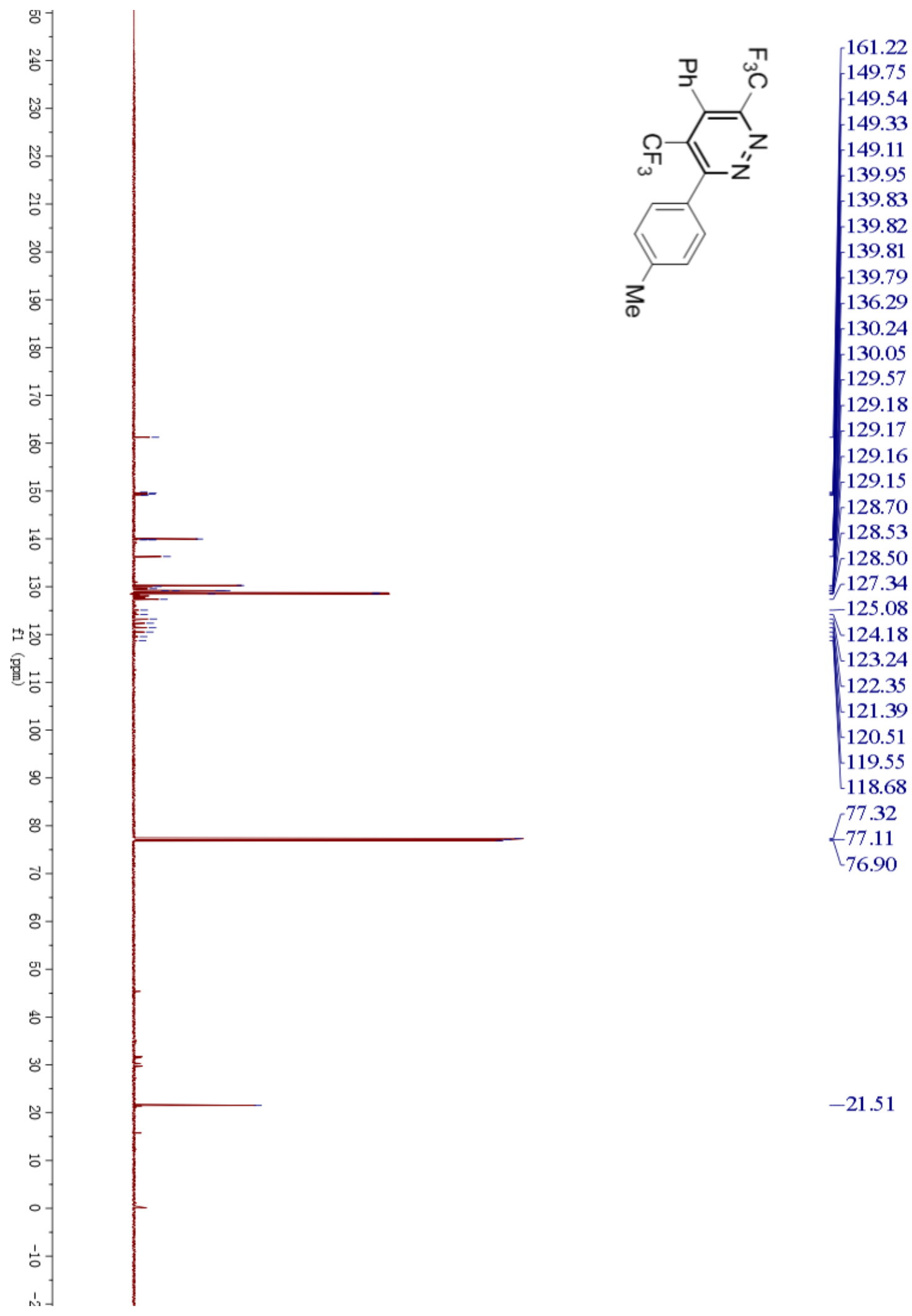


${ }^{19} \mathrm{~F} \mathrm{NMR}\left(565 \mathrm{MHz}, \mathrm{CDCl}_{3}\right)$ spectrum of $\mathbf{6 c}$

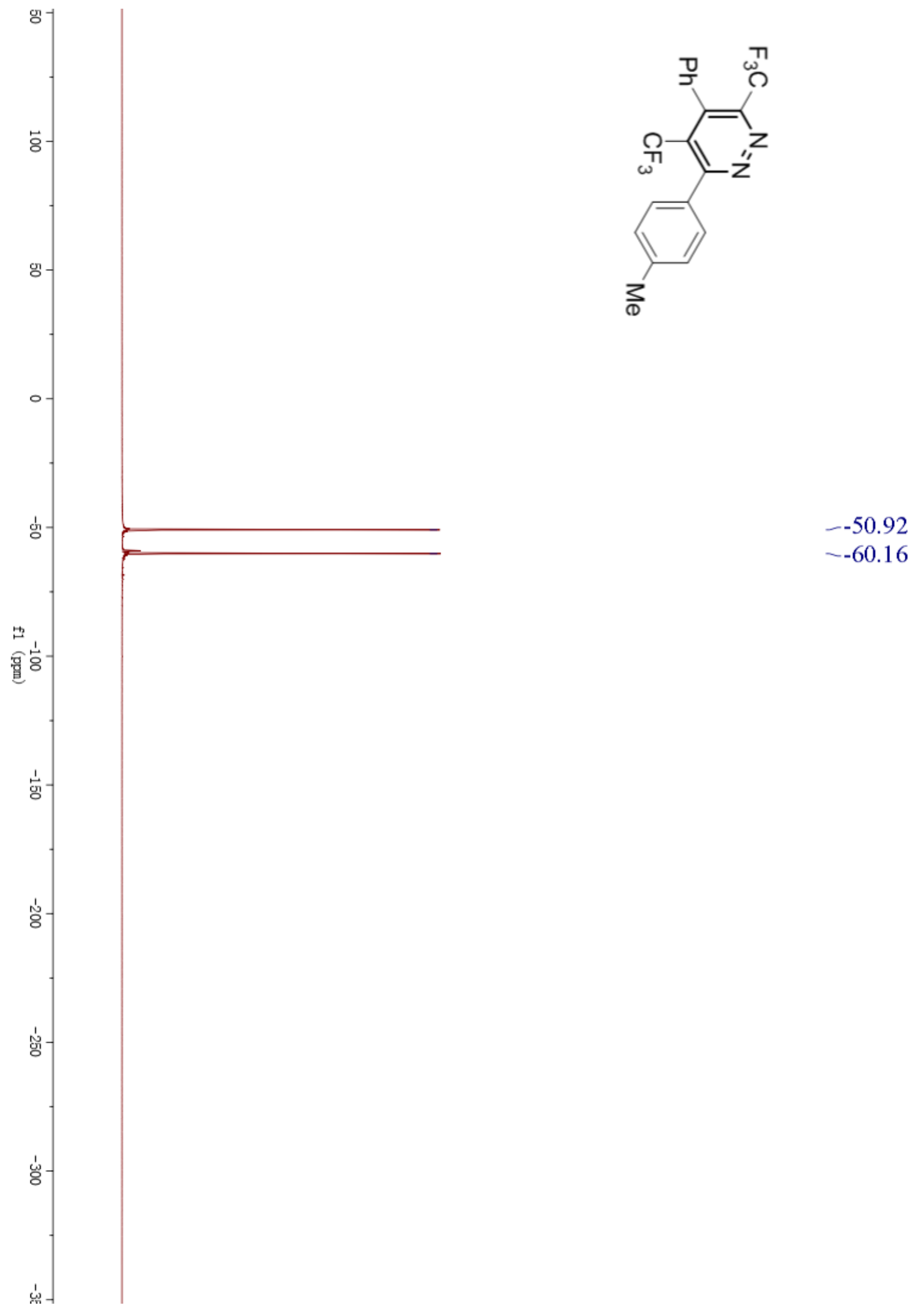


${ }^{1} \mathrm{H}$ NMR $\left(600 \mathrm{MHz}, \mathrm{CDCl}_{3}\right)$ spectrum of $\mathbf{6 d}$

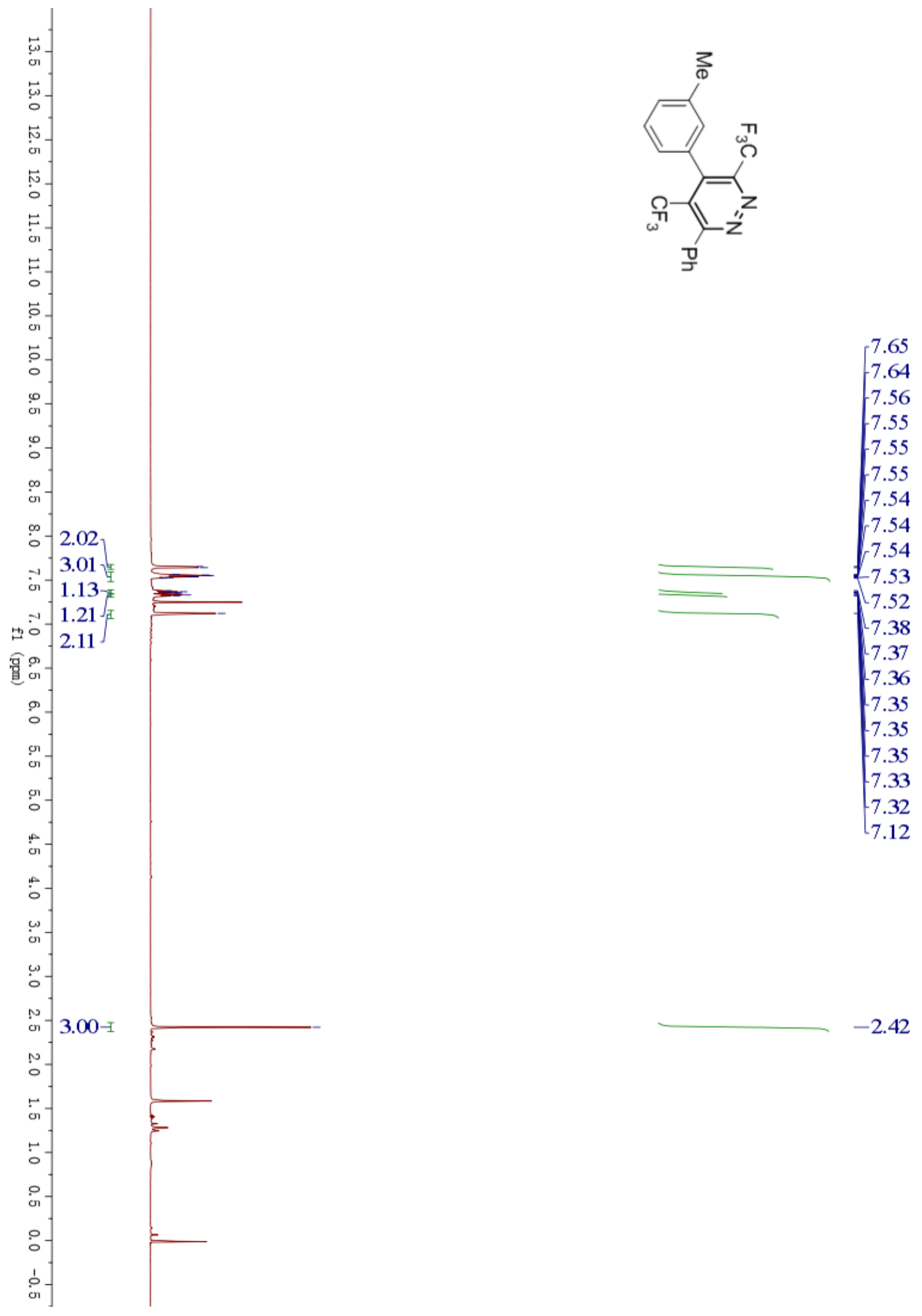


${ }^{13} \mathrm{C} \mathrm{NMR}\left(151 \mathrm{MHz}, \mathrm{CDCl}_{3}\right)$ spectrum of $\mathbf{6 d}$

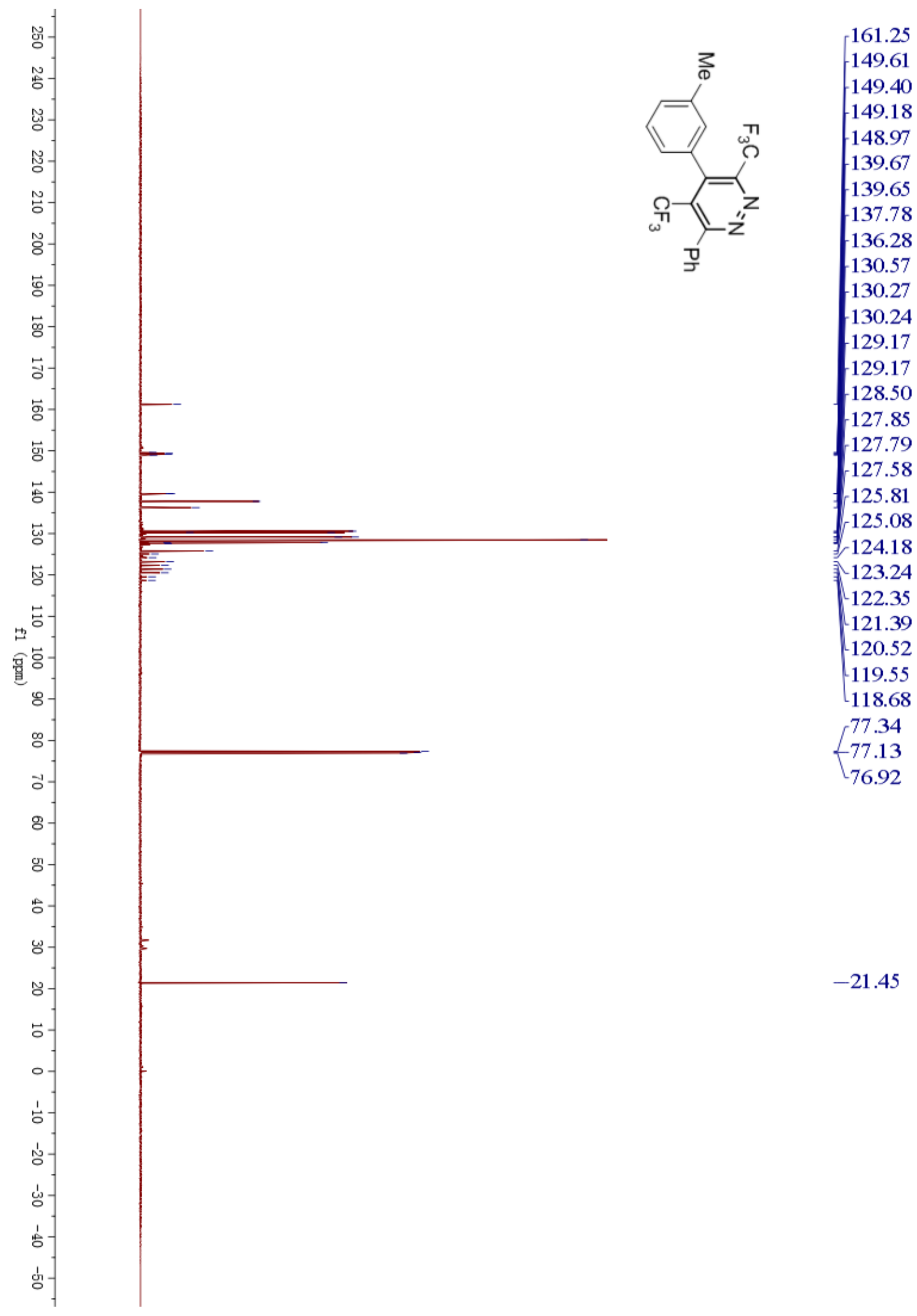


${ }^{19} \mathrm{~F}$ NMR $\left(565 \mathrm{MHz}, \mathrm{CDCl}_{3}\right)$ spectrum of $\mathbf{6 d}$

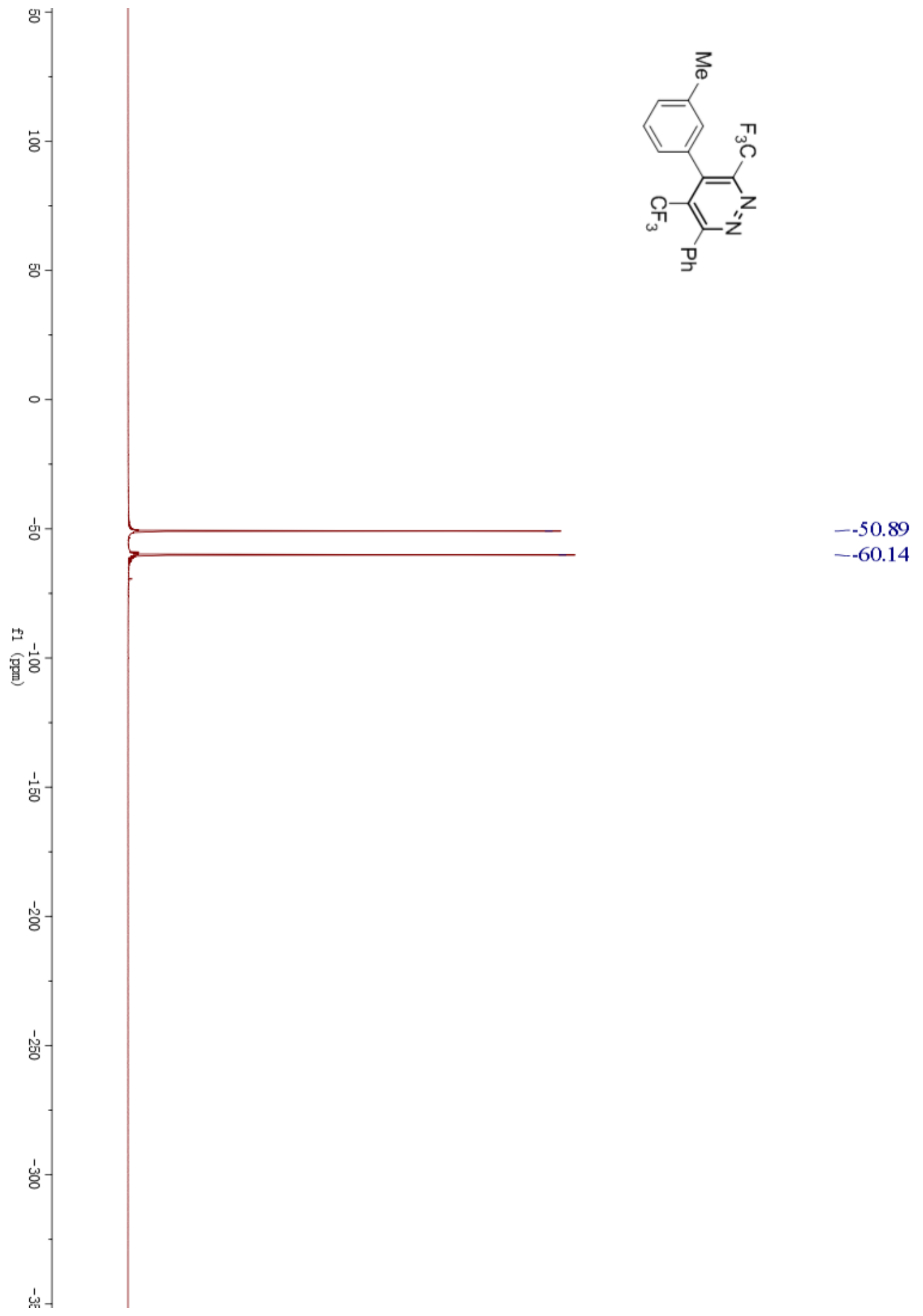


${ }^{1} \mathrm{H} \mathrm{NMR}\left(600 \mathrm{MHz}, \mathrm{CDCl}_{3}\right)$ spectrum of $\mathbf{6 e}$
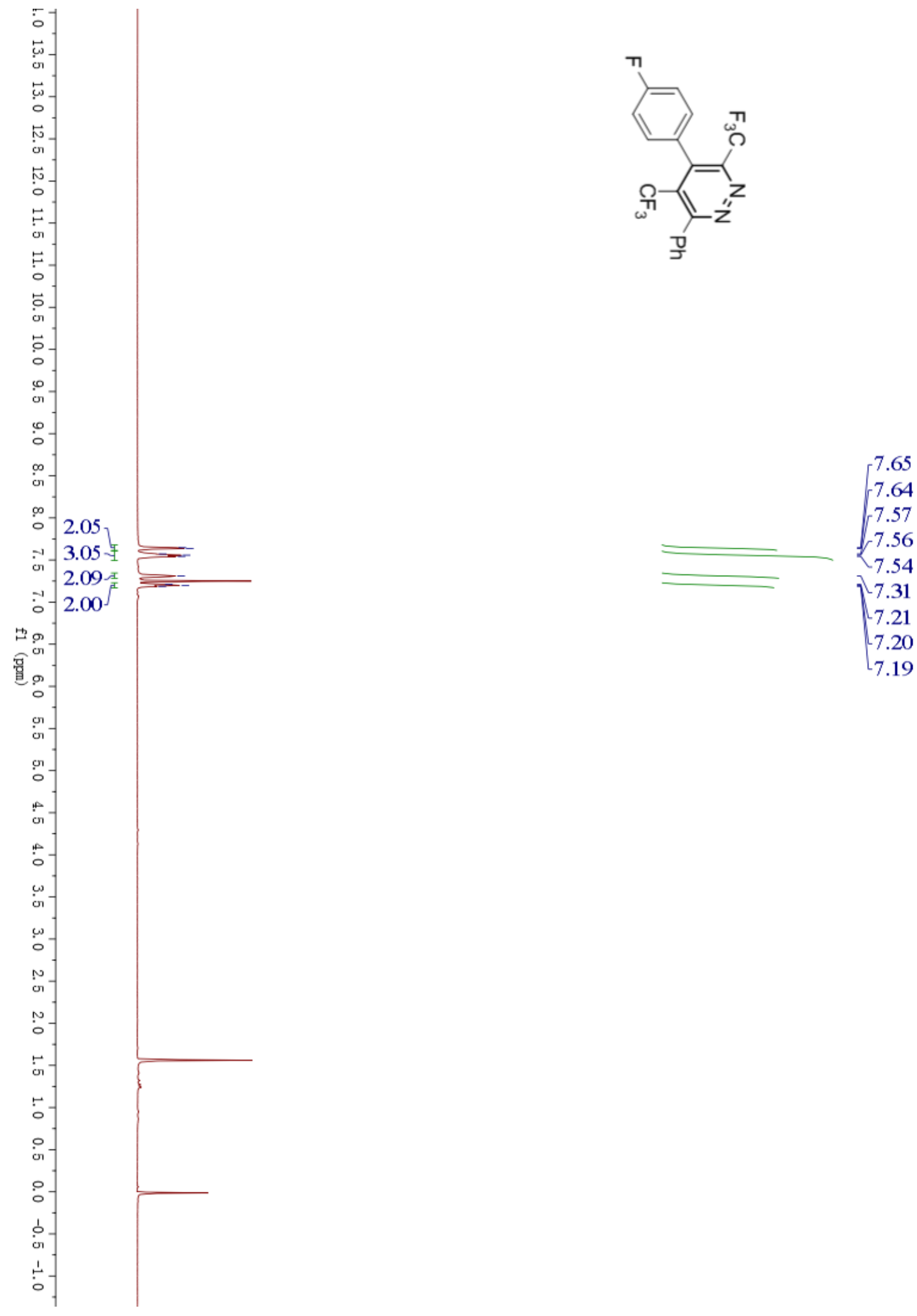
${ }^{13} \mathrm{C} \mathrm{NMR}\left(151 \mathrm{MHz}, \mathrm{CDCl}_{3}\right)$ spectrum of $\mathbf{6 e}$

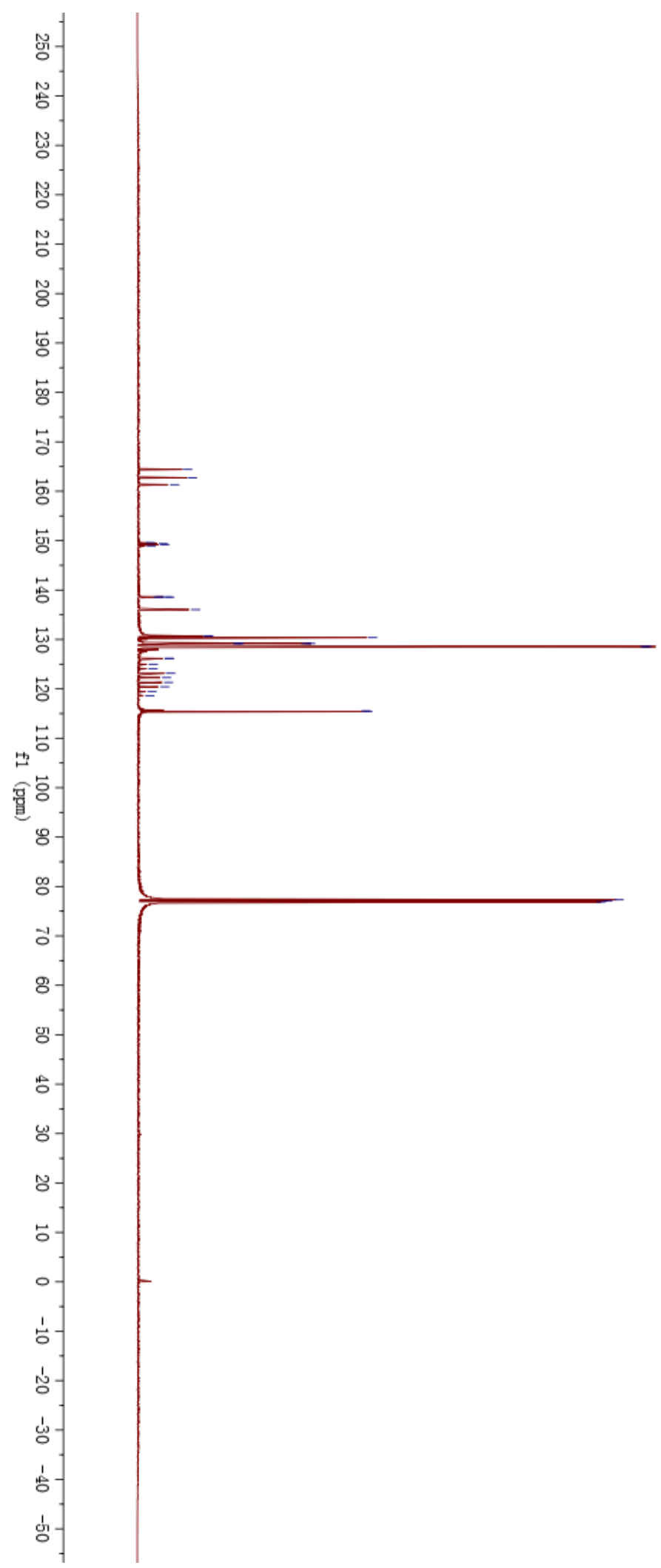

164.40

$-162.74$

161.33

$-149.38$

$-149.17$

138.60

138.59

138.58

$-138.56$

$-136.04$

$-130.72$

$-130.66$

$-130.38$

$-129.19$

129.18

$-129.16$

129.15

128.55

128.55

126.17

126.14

123.16

122.27

$-121.31$

$-120.44$

$-115.55$

11540

$-77.11$

76.90 
${ }^{19} \mathrm{~F}$ NMR (565 MHz, $\mathrm{CDCl}_{3}$ ) spectrum of $\mathbf{6 e}$

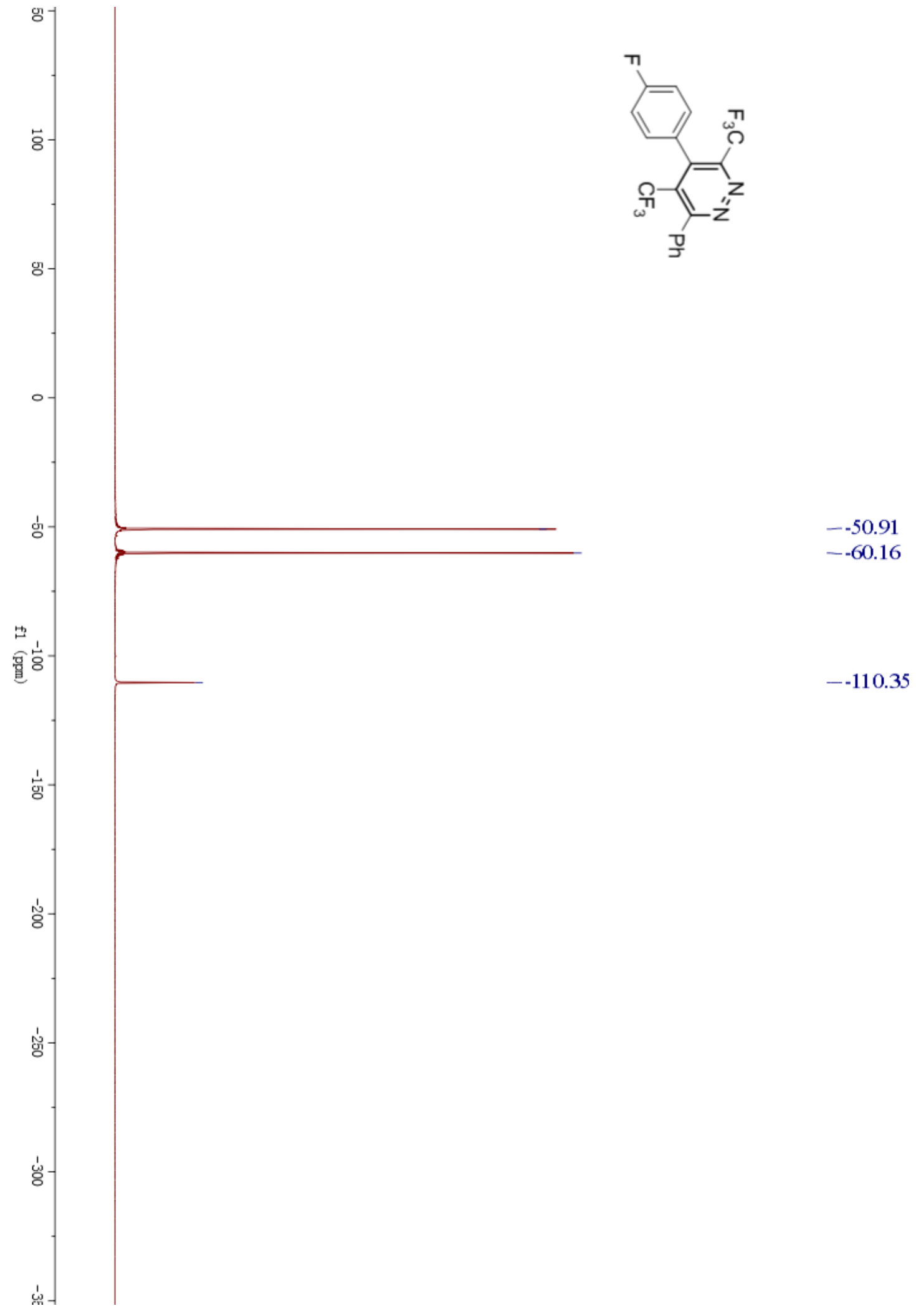


${ }^{1} \mathrm{H}$ NMR (600 MHz, $\left.\mathrm{CDCl}_{3}\right)$ spectrum of $\mathbf{6 f}$

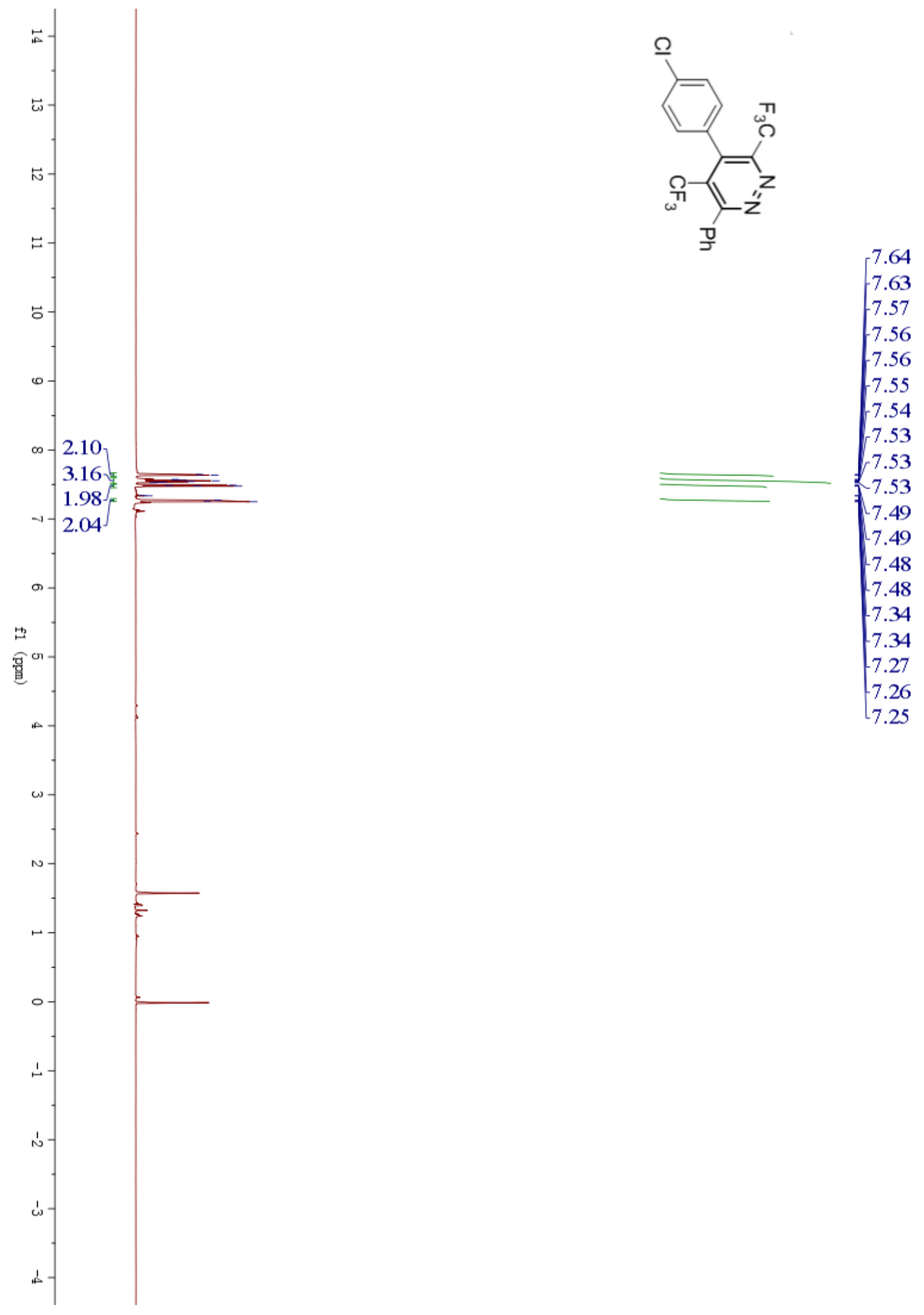


${ }^{13} \mathrm{C} \mathrm{NMR}\left(151 \mathrm{MHz}, \mathrm{CDCl}_{3}\right)$ spectrum of $\mathbf{6} \mathbf{f}$
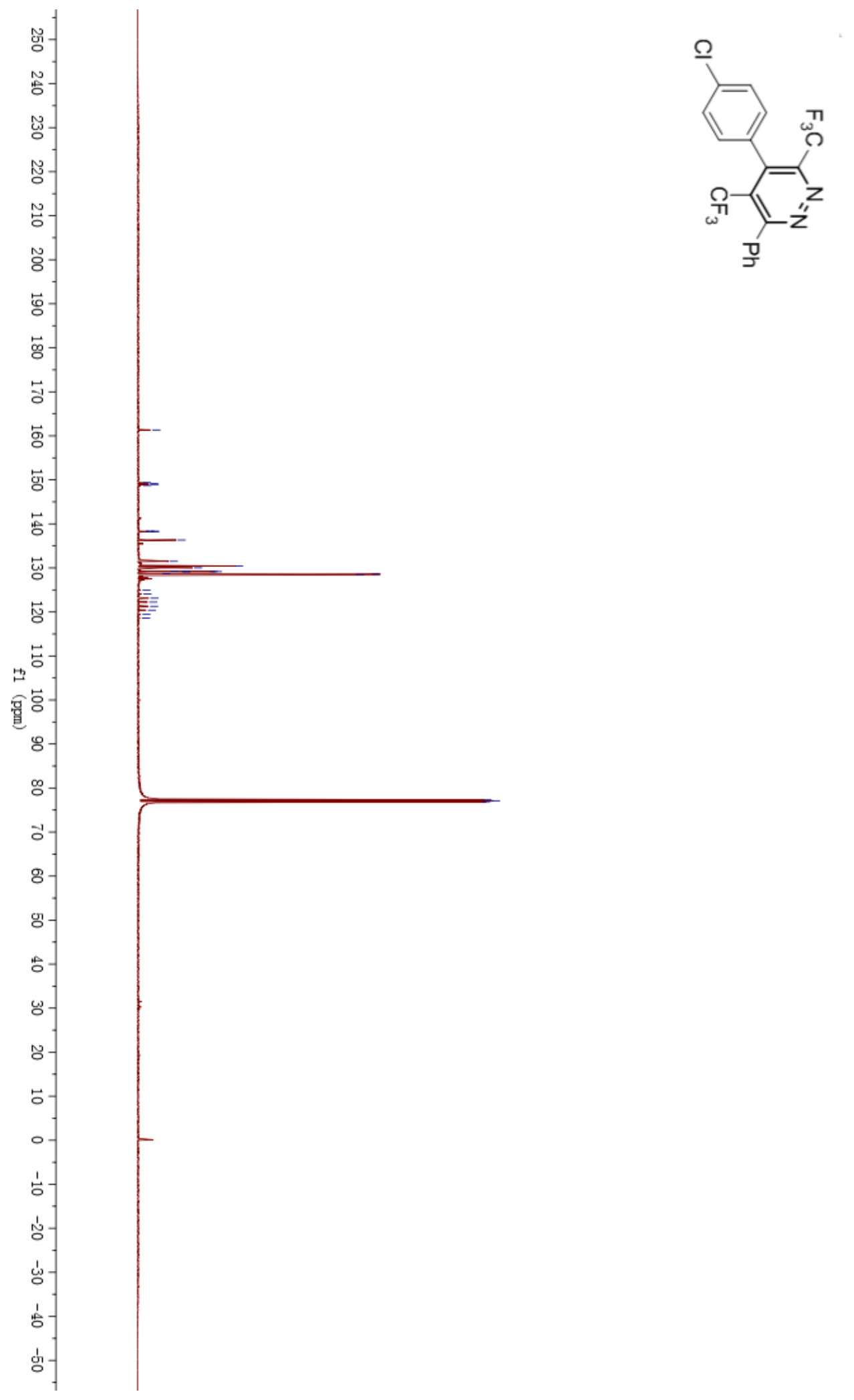

161.34

$-149.38$

149.17

148.95

148.74

138.31

138.30

138.28

138.27

136.33

131.50

$-130.42$

$-130.02$

$-129.27$

129.19

$-129.18$

129.17

129.16

128.72

$-128.57$

128.49

124.97

124.07

123.12

122.23

$-121.28$

$1720^{40}$

$-77.11$

76.90 
${ }^{19} \mathrm{~F}$ NMR (565 MHz, $\left.\mathrm{CDCl}_{3}\right)$ spectrum of $\mathbf{6 f}$

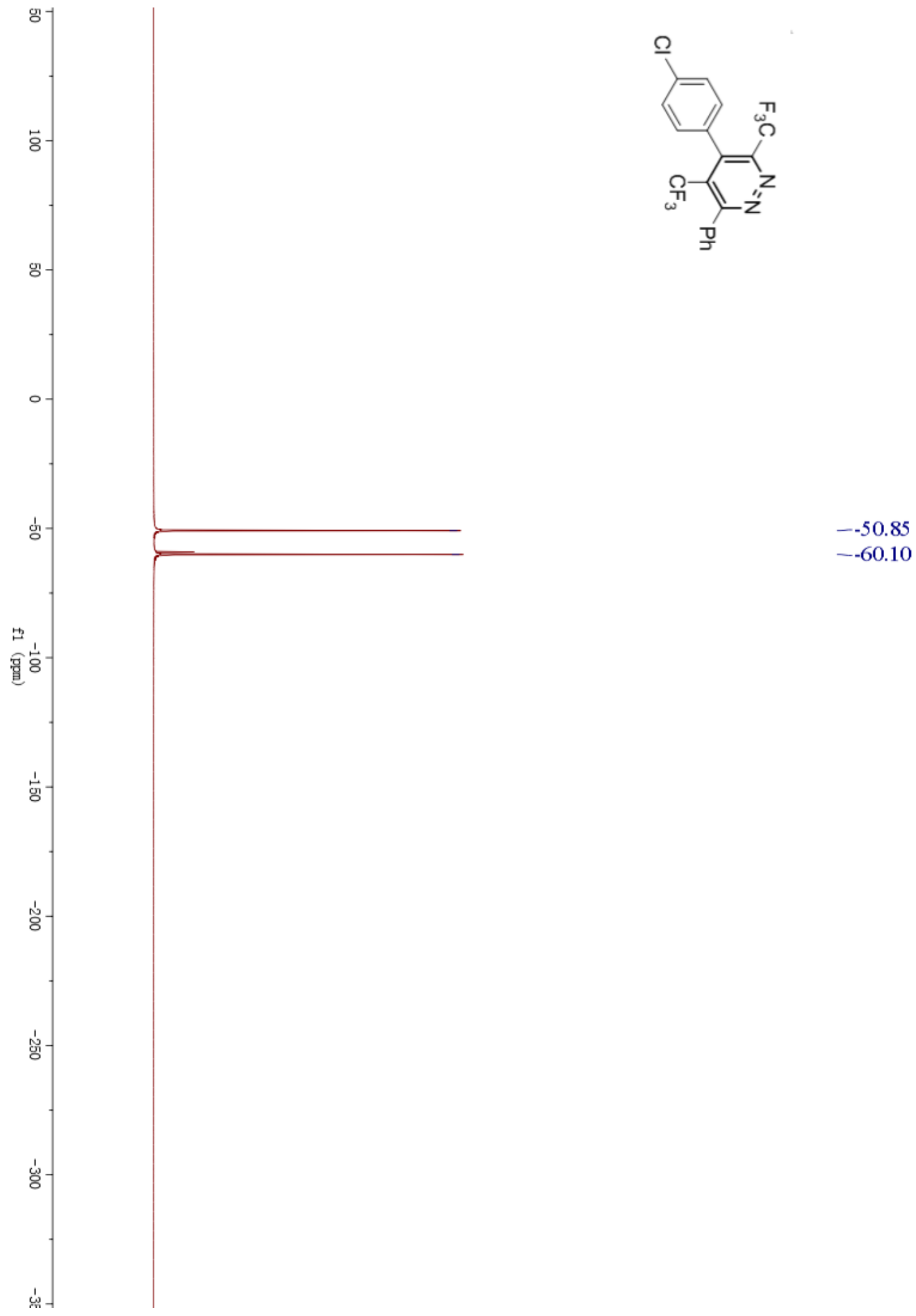


${ }^{1} \mathrm{H}$ NMR (600 MHz, $\left.\mathrm{CDCl}_{3}\right)$ spectrum of $\mathbf{6 g}$

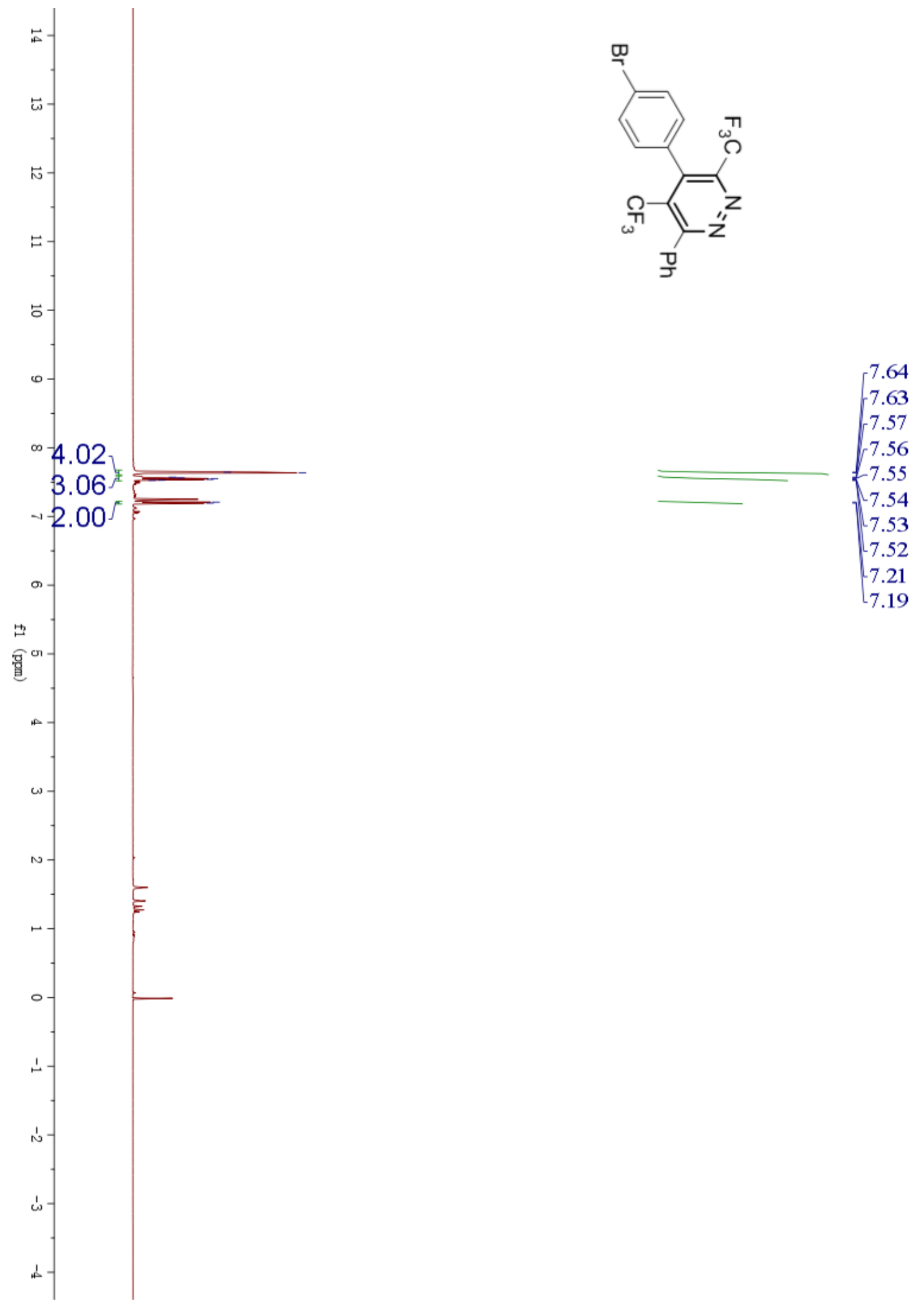


${ }^{13} \mathrm{C}$ NMR (151 MHz, $\mathrm{CDCl}_{3}$ ) spectrum of $\mathbf{6 g}$

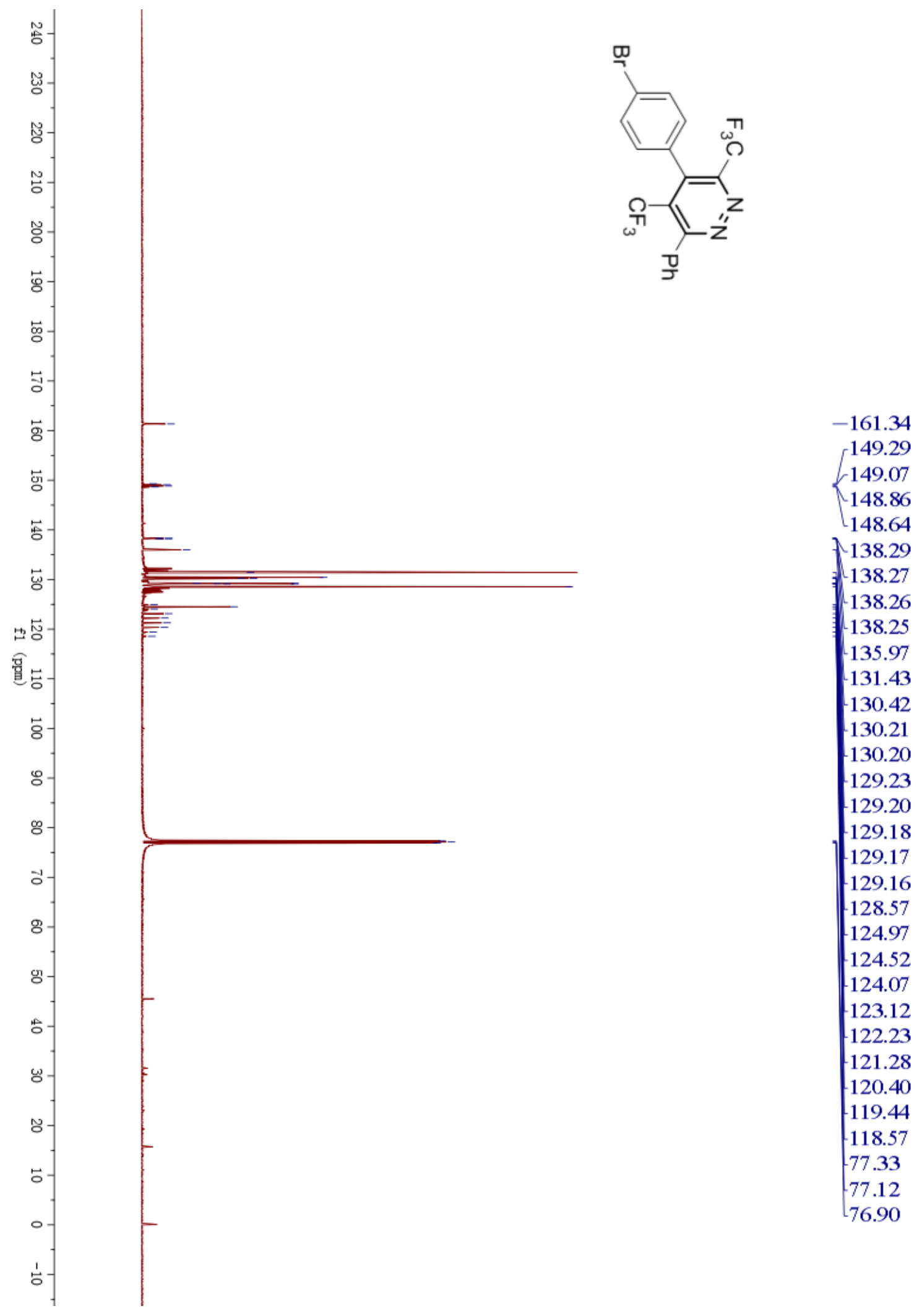


${ }^{19} \mathrm{~F} \mathrm{NMR}\left(565 \mathrm{MHz}, \mathrm{CDCl}_{3}\right)$ spectrum of $\mathbf{6 g}$

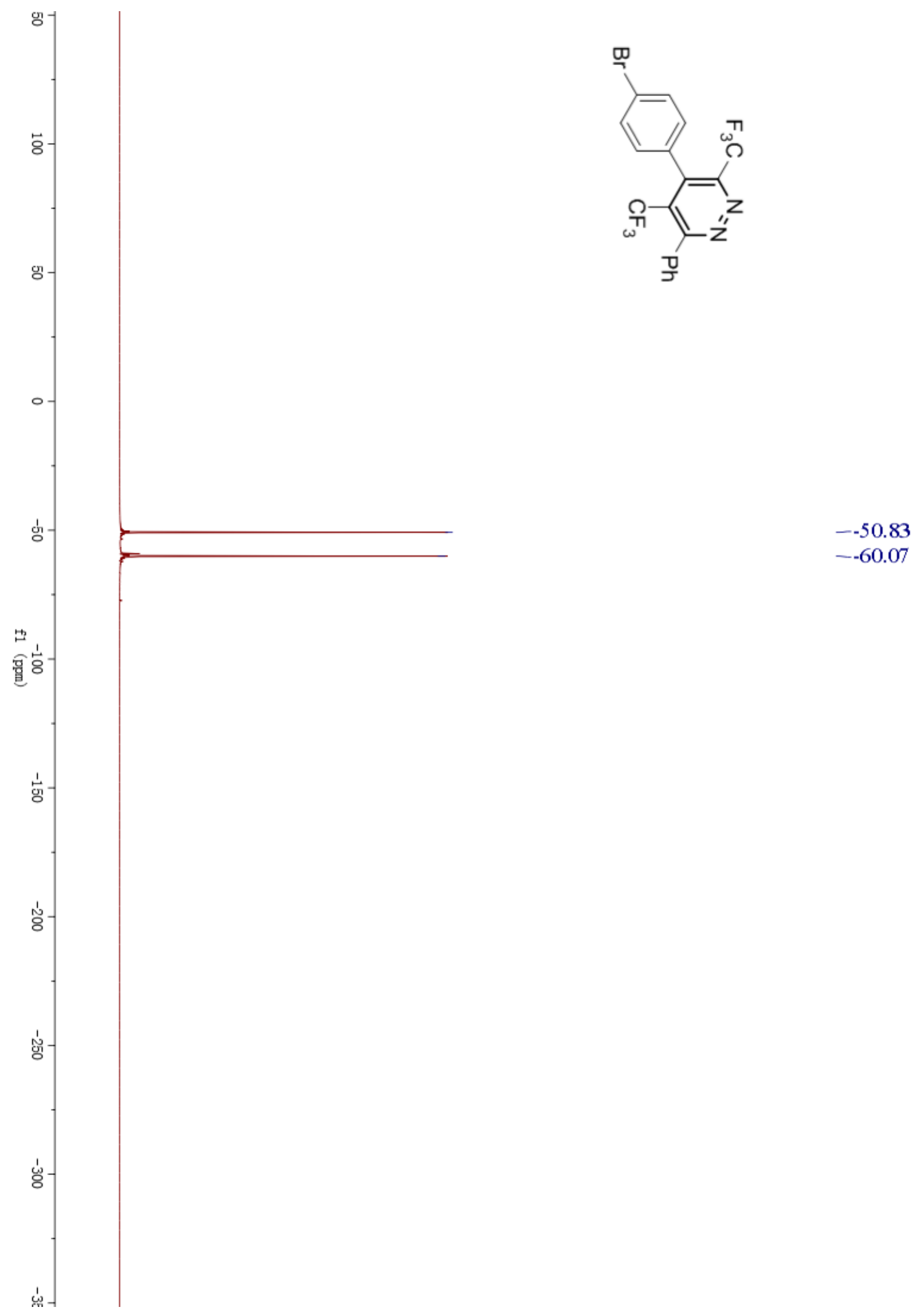


${ }^{1} \mathrm{H}$ NMR (600 MHz, $\mathrm{CDCl}_{3}$ ) spectrum of 7
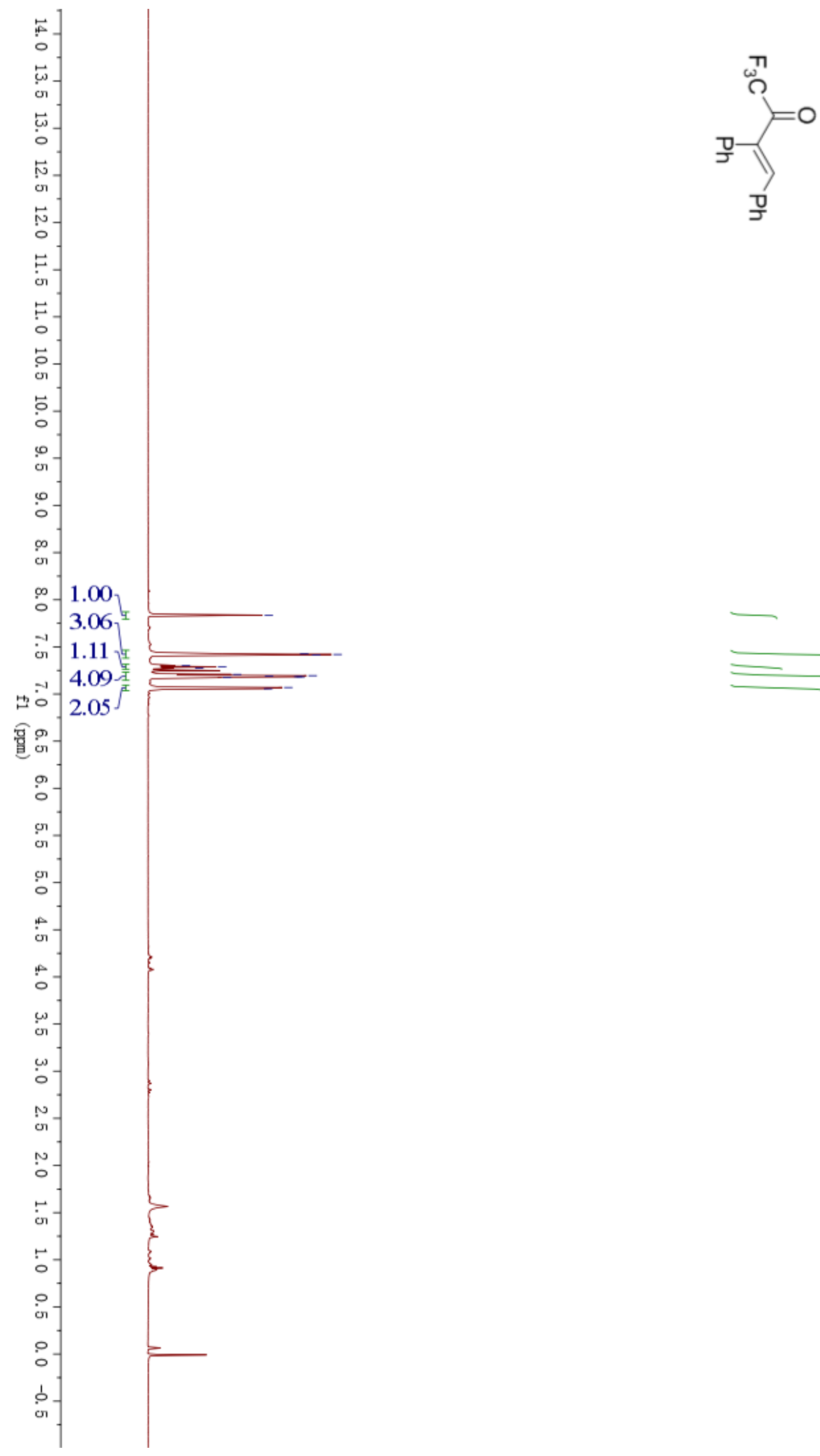
${ }^{13} \mathrm{C} \mathrm{NMR}\left(151 \mathrm{MHz}, \mathrm{CDCl}_{3}\right)$ spectrum of 7

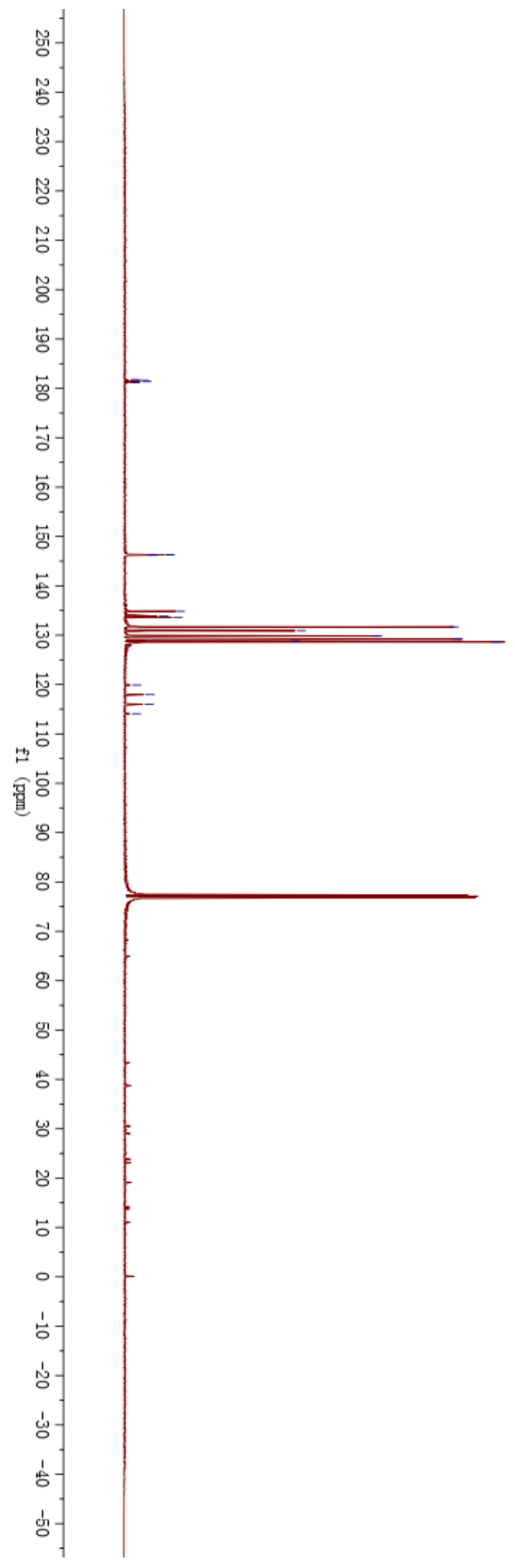

181.83

181.60

181.38

181.16

146.29

146.28

146.25

$\perp_{146.23}$

134.86

133.82

133.64

131.67

130.88

129.86

$-129.19$

128.82

128.62

119.89

117.95

$-116.02$

114.08 
${ }^{19} \mathrm{~F} \mathrm{NMR}\left(565 \mathrm{MHz}, \mathrm{CDCl}_{3}\right)$ spectrum of 7

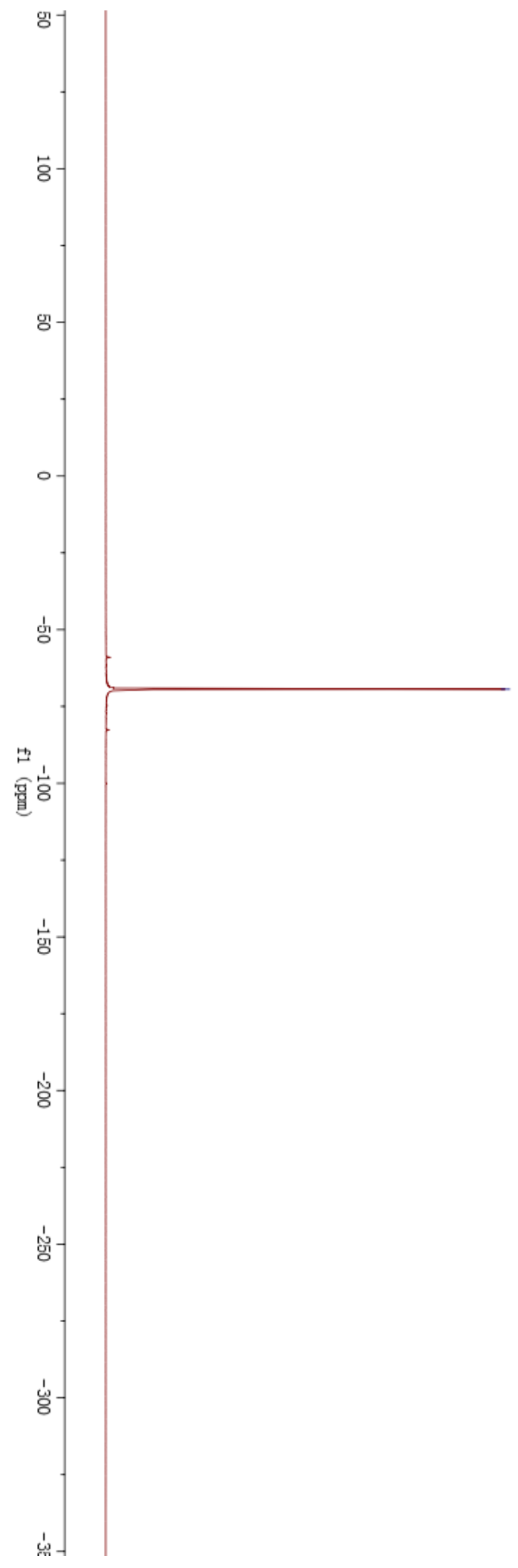




\section{X-Ray crystallographic data}

For the crystallization process, vacuum dried pure sample $\mathbf{2 a}$, or $\mathbf{2 e}$, or $\mathbf{2 p}$, or $\mathbf{5 a}$, or 6a (around 50-80 mg) was taken in a vial and dissolved in $1 \mathrm{~mL}$ of DCM and $5 \mathrm{~mL}$ hexane. The vial was kept around room temperature for slow evaporation. After 1-2 days, we could find colorless, needle shape crystal, which was picked up from the vial and performed single crystal XRD study.

The X-ray crystallographic structure for 2a. Crystal data has been deposited to CCDC, number 2086388.

\begin{tabular}{|c|c|}
\hline Empirical formula & $\mathrm{C}_{18} \mathrm{H}_{13} \mathrm{~F}_{6} \mathrm{~N}_{3}$ \\
\hline Formula weight & 385.31 \\
\hline Temperature/K & 293(2) \\
\hline Crystal system & monoclinic \\
\hline Space group & $\mathrm{P} 21 / \mathrm{n}$ \\
\hline $\mathrm{a} / \AA$ & $9.0456(13)$ \\
\hline $\mathrm{b} / \AA$ & $18.517(3)$ \\
\hline $\mathrm{c} / \AA$ & $11.4043(19)$ \\
\hline$\alpha /{ }^{\circ}$ & 90 \\
\hline$\beta /{ }^{\circ}$ & $113.216(19)$ \\
\hline$\gamma /{ }^{\circ}$ & 90 \\
\hline Volume $/ \AA^{3}$ & $1755.5(5)$ \\
\hline $\mathrm{Z}$ & 4 \\
\hline$\rho_{\text {calcg }} / \mathrm{cm}^{3}$ & 1.458 \\
\hline$\mu / \mathrm{mm}^{-1}$ & 0.133 \\
\hline $\mathrm{F}(000)$ & 784.0 \\
\hline Crystal size $/ \mathrm{mm}^{3}$ & $0.28 \times 0.22 \times 0.12$ \\
\hline Radiation & $\operatorname{Mo} \operatorname{K} \alpha(\lambda=0.71073)$ \\
\hline $2 \Theta$ range for data collection $/{ }^{\circ}$ & 4.4 to 59 \\
\hline Index ranges & $-12 \leq \mathrm{h} \leq 10,-24 \leq \mathrm{k} \leq 23,-14 \leq 1 \leq 15$ \\
\hline Reflections collected & 12399 \\
\hline Independent reflections & $3975\left[\mathrm{R}_{\text {int }}=0.0355, \mathrm{R}_{\text {sigma }}=0.0522\right]$ \\
\hline Data/restraints/parameters & $3975 / 0 / 247$ \\
\hline Goodness-of-fit on $\mathrm{F}^{2}$ & 1.081 \\
\hline Final $\mathrm{R}$ indexes $[\mathrm{I}>=2 \sigma(\mathrm{I})]$ & $\mathrm{R}_{1}=0.0652, \mathrm{wR}_{2}=0.1645$ \\
\hline Final $\mathrm{R}$ indexes [all data] & $\mathrm{R}_{1}=0.1194, \mathrm{wR}_{2}=0.1825$ \\
\hline Largest diff. peak/hole / e $\AA^{-3}$ & $0.46 /-0.42$ \\
\hline
\end{tabular}


The X-ray crystallographic structure for 2e. Crystal data has been deposited to CCDC, number 2086389.

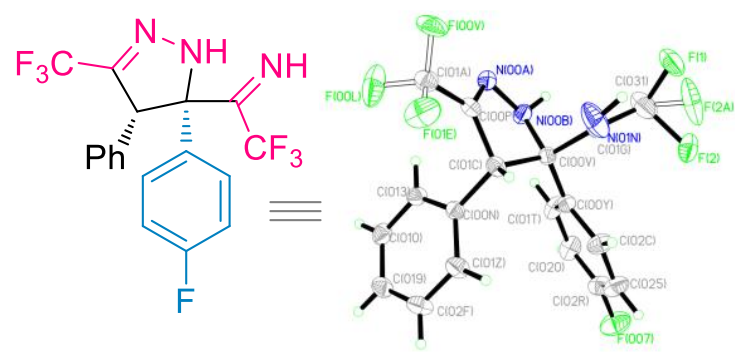

\begin{tabular}{|c|c|}
\hline Empirical formula & $\mathrm{C}_{18} \mathrm{H}_{12} \mathrm{~F}_{7} \mathrm{~N}_{3}$ \\
\hline Formula weight & 403.31 \\
\hline Temperature/K & 296.15 \\
\hline Crystal system & orthorhombic \\
\hline Space group & Pbca \\
\hline $\mathrm{a} / \AA$ & $9.4311(10)$ \\
\hline $\mathrm{b} / \AA$ & 16.9922(19) \\
\hline $\mathrm{c} / \AA$ & $22.113(3)$ \\
\hline$\alpha /{ }^{\circ}$ & 90 \\
\hline$\beta /^{\circ}$ & 90 \\
\hline$\gamma /{ }^{\circ}$ & 90 \\
\hline Volume $/ \AA^{3}$ & $3543.7(7)$ \\
\hline $\mathrm{Z}$ & 8 \\
\hline$\rho_{\text {calc }} \mathrm{g} / \mathrm{cm}^{3}$ & 1.512 \\
\hline$\mu / \mathrm{mm}^{-1}$ & 0.143 \\
\hline $\mathrm{F}(000)$ & 1632.0 \\
\hline Crystal size $/ \mathrm{mm}^{3}$ & $0.32 \times 0.24 \times 0.16$ \\
\hline Radiation & $\operatorname{MoK} \alpha(\lambda=0.71073)$ \\
\hline $2 \Theta$ range for data collection ${ }^{\circ}$ & 4.794 to 50.052 \\
\hline Index ranges & $-11 \leq \mathrm{h} \leq 11,-20 \leq \mathrm{k} \leq 20,-26 \leq 1 \leq 26$ \\
\hline Reflections collected & 51436 \\
\hline Independent reflections & $3129\left[\mathrm{R}_{\text {int }}=0.1229, \mathrm{R}_{\text {sigma }}=0.0484\right]$ \\
\hline Data/restraints/parameters & $3129 / 115 / 313$ \\
\hline Goodness-of-fit on $\mathrm{F}^{2}$ & 1.028 \\
\hline Final $R$ indexes $[I>=2 \sigma(I)]$ & $\mathrm{R}_{1}=0.0576, \mathrm{wR}_{2}=0.1376$ \\
\hline Final $\mathrm{R}$ indexes [all data] & $\mathrm{R}_{1}=0.1094, \mathrm{wR}_{2}=0.1631$ \\
\hline Largest diff. peak/hole / e $\AA^{-3}$ & $0.34 /-0.27$ \\
\hline
\end{tabular}


The X-ray crystallographic structure for $\mathbf{2 p .}$ Crystal data has been deposited to CCDC, number 2086392.

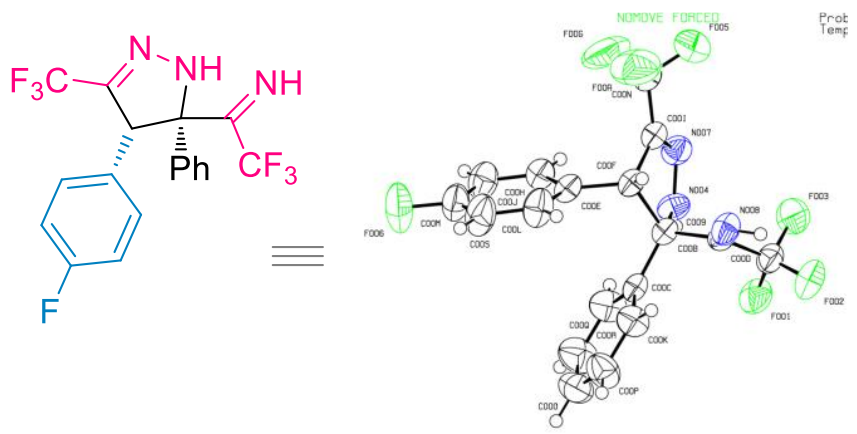

\begin{tabular}{|c|c|}
\hline Empirical formula & $\mathrm{C}_{18} \mathrm{H}_{11} \mathrm{~F}_{7} \mathrm{~N}_{3}$ \\
\hline Formula weight & 402.30 \\
\hline Temperature/K & 293.15 \\
\hline Crystal system & orthorhombic \\
\hline Space group & $\mathrm{P} 2{ }_{1} 2{ }_{1}{ }_{1}$ \\
\hline $\mathrm{a} / \AA$ & $5.9754(14)$ \\
\hline $\mathrm{b} / \AA$ & $15.824(3)$ \\
\hline$c / \AA$ & $22.222(5)$ \\
\hline$\alpha /{ }^{\circ}$ & 90 \\
\hline$\beta /^{\circ}$ & 90 \\
\hline$\gamma /{ }^{\circ}$ & 90 \\
\hline Volume/ $/ \AA^{3}$ & 2101.2(8) \\
\hline $\mathrm{Z}$ & 4 \\
\hline$\rho_{\text {calc }} / \mathrm{cm}^{3}$ & 1.272 \\
\hline$\mu / \mathrm{mm}^{-1}$ & 0.121 \\
\hline $\mathrm{F}(000)$ & 812.0 \\
\hline Crystal size $/ \mathrm{mm}^{3}$ & $0.32 \times 0.14 \times 0.12$ \\
\hline Radiation & $\operatorname{Mo} \operatorname{K} \alpha(\lambda=0.71073)$ \\
\hline $2 \Theta$ range for data collection $/{ }^{\circ}$ & 3.666 to 50.05 \\
\hline Index ranges & $-7 \leq \mathrm{h} \leq 6,-17 \leq \mathrm{k} \leq 18,-25 \leq 1 \leq 26$ \\
\hline Reflections collected & 12111 \\
\hline Independent reflections & $3653\left[R_{\text {int }}=0.0537, R_{\text {sigma }}=0.0593\right]$ \\
\hline Data/restraints/parameters & $3653 / 1 / 257$ \\
\hline Goodness-of-fit on $\mathrm{F}^{2}$ & 1.077 \\
\hline Final $R$ indexes $[\mathrm{I}>=2 \sigma(\mathrm{I})]$ & $\mathrm{R}_{1}=0.0541, \mathrm{wR}_{2}=0.1433$ \\
\hline Final $\mathrm{R}$ indexes [all data] & $\mathrm{R}_{1}=0.0709, \mathrm{wR}_{2}=0.1523$ \\
\hline Largest diff. peak/hole / e $\AA^{-3}$ & $0.34 /-0.24$ \\
\hline Flack parameter & $0.5(14)$ \\
\hline
\end{tabular}


The X-ray crystallographic structure for 5a. Crystal data has been deposited to CCDC, number 2086393.

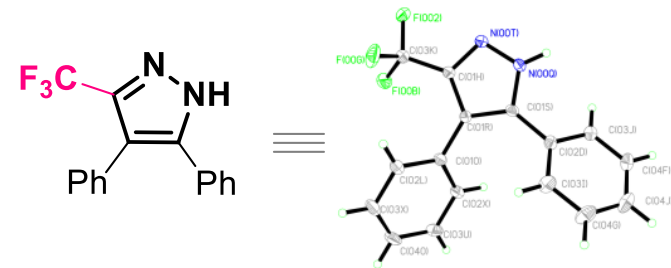

\begin{tabular}{|c|c|}
\hline Empirical formula & $\mathrm{C}_{18} \mathrm{H}_{10} \mathrm{~F}_{6} \mathrm{~N}_{2}$ \\
\hline Formula weight & 368.28 \\
\hline Temperature/K & 293.15 \\
\hline Crystal system & monoclinic \\
\hline Space group & $\mathrm{P} 2{ }_{1}$ \\
\hline $\mathrm{a} / \AA$ & $10.414(4)$ \\
\hline $\mathrm{b} / \AA$ & 7.3734(16) \\
\hline$c / \AA$ & $11.322(3)$ \\
\hline$\alpha /{ }^{\circ}$ & 90 \\
\hline$\beta /{ }^{\circ}$ & $110.75(3)$ \\
\hline$\gamma /{ }^{\circ}$ & 90 \\
\hline Volume $/ \AA^{3}$ & $813.0(4)$ \\
\hline $\mathrm{Z}$ & 2 \\
\hline$\rho_{\text {calc }} g / \mathrm{cm}^{3}$ & 1.504 \\
\hline$\mu / \mathrm{mm}^{-1}$ & 0.138 \\
\hline $\mathrm{F}(000)$ & 372.0 \\
\hline Crystal size $/ \mathrm{mm}^{3}$ & $0.23 \times 0.18 \times 0.13$ \\
\hline Radiation & $\operatorname{Mo~} \mathrm{K} \alpha(\lambda=0.71073)$ \\
\hline $2 \Theta$ range for data collection $/^{\circ}$ & 3.846 to 50.052 \\
\hline Index ranges & $-11 \leq \mathrm{h} \leq 12,-8 \leq \mathrm{k} \leq 8,-13 \leq 1 \leq 12$ \\
\hline Reflections collected & 5568 \\
\hline Independent reflections & $2591\left[\mathrm{R}_{\text {int }}=0.0821, \mathrm{R}_{\text {sigma }}=0.1384\right]$ \\
\hline Data/restraints/parameters & $2591 / 1 / 212$ \\
\hline Goodness-of-fit on $\mathrm{F}^{2}$ & 0.956 \\
\hline Final $\mathrm{R}$ indexes $[\mathrm{I}>=2 \sigma(\mathrm{I})]$ & $\mathrm{R}_{1}=0.0679, \mathrm{wR}_{2}=0.1463$ \\
\hline Final $\mathrm{R}$ indexes [all data] & $\mathrm{R}_{1}=0.1383, \mathrm{wR}_{2}=0.1747$ \\
\hline Largest diff. peak/hole / e $\AA^{-3}$ & $0.18 /-0.19$ \\
\hline Flack parameter & $1(2)$ \\
\hline
\end{tabular}


The X-ray crystallographic structure for 6a. Crystal data has been deposited to CCDC, number 2086394.

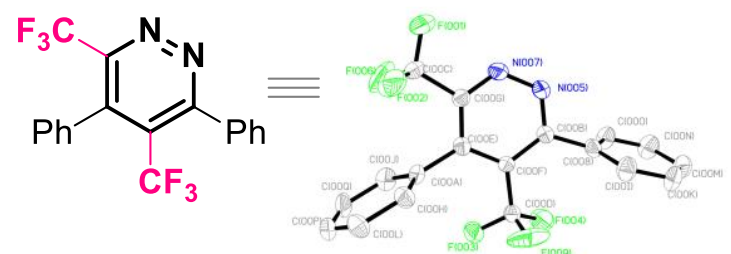

\begin{tabular}{|c|c|}
\hline Empirical formula & $\mathrm{C}_{16} \mathrm{H}_{11} \mathrm{~F}_{3} \mathrm{~N}_{2}$ \\
\hline Formula weight & 288.27 \\
\hline Temperature/K & 296.15 \\
\hline Crystal system & monoclinic \\
\hline Space group & $\mathrm{P} 2 / \mathrm{c}$ \\
\hline $\mathrm{a} / \AA ̊$ & $39.974(2)$ \\
\hline $\mathrm{b} / \AA$ & $13.8880(7)$ \\
\hline$c / \AA$ & $20.2594(12)$ \\
\hline$\alpha /{ }^{\circ}$ & 90 \\
\hline$\beta /{ }^{\circ}$ & $96.119(2)$ \\
\hline$\gamma /{ }^{\circ}$ & 90 \\
\hline Volume $/ \AA^{3}$ & $11183.1(10)$ \\
\hline $\mathrm{Z}$ & 32 \\
\hline$\rho_{\text {calc }} g / \mathrm{cm}^{3}$ & 1.370 \\
\hline$\mu / \mathrm{mm}^{-1}$ & 0.110 \\
\hline $\mathrm{F}(000)$ & 4736.0 \\
\hline Crystal size $/ \mathrm{mm}^{3}$ & $0.19 \times 0.16 \times 0.12$ \\
\hline Radiation & $\operatorname{MoK} \alpha(\lambda=0.71073)$ \\
\hline $2 \Theta$ range for data collection $/{ }^{\circ}$ & 4 to 50.054 \\
\hline Index ranges & $-47 \leq \mathrm{h} \leq 47,-16 \leq \mathrm{k} \leq 16,-24 \leq 1 \leq 24$ \\
\hline Reflections collected & 174133 \\
\hline Independent reflections & $19747\left[R_{\text {int }}=0.1043, R_{\text {sigma }}=0.0533\right]$ \\
\hline Data/restraints/parameters & $19747 / 0 / 1513$ \\
\hline Goodness-of-fit on $\mathrm{F}^{2}$ & 1.017 \\
\hline Final $R$ indexes $[I>=2 \sigma(I)]$ & $\mathrm{R}_{1}=0.0565, \mathrm{wR}_{2}=0.1287$ \\
\hline Final $\mathrm{R}$ indexes [all data] & $\mathrm{R}_{1}=0.0980, \mathrm{wR}_{2}=0.1496$ \\
\hline Largest diff. peak/hole / e $\AA^{-3}$ & $0.65 /-0.57$ \\
\hline
\end{tabular}

